\title{
Characterization of laminar separation bubbles using infrared thermography
}

\author{
by \\ Dallyn Walter Wynnychuk
}

\author{
A thesis \\ presented to the University of Waterloo \\ in fulfillment of the \\ thesis requirement for the degree of \\ Master of Applied Science \\ in \\ Mechanical Engineering
}

Waterloo, Ontario, Canada, 2019

(C) Dallyn Walter Wynnychuk 2019 
I hereby declare that I am the sole author of this thesis. This is a true copy of the thesis, including any required final revisions, as accepted by my examiners.

I understand that my thesis may be made electronically available to the public. 


\begin{abstract}
An experimental investigation of laminar separation bubbles is presented, demonstrating the feasibility of employing infrared thermography for identification and quantitative characterization of a laminar separation bubble. The investigation is comprised of a series of wind tunnel tests performed on two main geometries. The first experimental investigation is performed on a flat plate, where a laminar separation bubble is formed upstream of a vertically mounted fence. Velocity field, surface pressure, and surface temperature results are presented from three main flow conditions, corresponding to fence height-based Reynolds numbers of 27,000,36,000, and 45,000. The second experimental investigation is performed on a NACA 0018 airfoil. Velocity field and surface temperature results are presented at two main flow conditions, pertaining to an angle of attack $\alpha=4^{\circ}$ and chordbased Reynolds numbers of 80,000 and 120,000. For each experimental geometry, velocity field measurements provide initial estimations of the size and location of laminar separation bubbles, while highlighting details regarding the transition process, which are used to formulate expected convective heat transfer variations on the model surface.

For both experimental investigations, locations of mean separation and transition are estimated where the streamwise gradient of surface temperature reaches a maximum and minimum value, respectively, while mean reattachment is estimated at the location of minimum surface temperature. Characterization of the separated region with this methodology is shown to reasonably agree with baseline estimates within experimental uncertainty. However, fundamental assumptions regarding uniformity of the applied radiative heat flux on the model surface are required. In this regard, a less restrictive representation of the convective heat transfer variation is obtained by performing a differential energy balance at the model surface, considering surface temperatures in both quiescent and flow conditions. The energy balance allows for an estimated streamwise variation of the convective heat transfer coefficient. The approach yields good agreement with reference measurements on both geometries, with higher-precision estimation attained in airfoil experiments since the airfoil model was designed to minimize wall-normal conduction in the material. Transient measurements of surface temperature decay following a change in radiative flux are also investigated and used to directly estimate the variation of convective heat transfer coefficient on the airfoil. The convective heat transfer coefficient is shown to follow the expected variation; however, significant noise propagation and relatively low temporal resolution adversely influence the results, such that transient-based characterization should be restricted to situations where quasi-steady state measurements are not possible, or key simplifying assumptions cannot be satisfied.
\end{abstract}




\section{Acknowledgements}

First and foremost, I would like to acknowledge my supervisor, Professor Serhiy Yarusevych, for the invaluable guidance he has provided throughout every stage of this endeavour. Your attention to detail and continuous push for excellence has allowed me to grow beyond my expectations and get the most out of my graduate studies. I am truly grateful for the opportunity to research under your supervision.

I would like to thank the fellow FMRL students which I had the pleasure of working with: Alex Stothart, Amit Dutta, Ben Pocock, Burak Tuna, Caddie Zhang, Erik Marble, Jeff McClure, John Kurelek, Jon Deng, Mark Istvan, Supun Pieris, Qihang Yuan, Winston $\mathrm{Hu}$, and Yash Shah, I appreciate the help with setting up experiments, the countless fluids related discussions, and the participation in our games of dodgeball. Amit and Yash, I am grateful for the help you provided with my coursework, especially with advanced fluid mechanics where it was needed most. John, I appreciate the many times you helped out with my experiments, and various Matlab related issues. Supun, I am thankful for your persistence in converting me to Python, and the countless times you helped me improve the quality of my figures along the way.

I would also like to extend thanks to the University of Waterloo staff members who provided assistance in the fluid mechanics lab. A special thanks is owed to Jason Benninger for the many times he was able to accommodate the ambitious schedules associated with preparing for experimental campaigns.

Thank you to my family who have provided support throughout every stage of my academic journey. To my parents, Michelle and Walter, my siblings, Ryan, Jade, and Trystan, and my extended family, your unconditional support and continuous encouragement has helped me through the most difficult times. Lastly, I would like to thank my friends, both new and old, who were able to take my mind off school when I needed it the most.

Financial support for this study was provided by the Ontario Graduate Scholarship program and the University of Waterloo. 


\section{Table of Contents}

List of Tables $\quad$ viii

List of Figures $\quad$ ix

Nomenclature $\quad$ xiv

1 Introduction $\quad 1$

1.1 Study Objectives ............................. 4

2 Background $\quad 5$

2.1 Laminar Separation Bubbles at Low Reynolds Numbers . . . . . . . . . . . 5

2.2 Measurement Techniques for Laminar Separation Bubbles . . . . . . . . . . 9

2.2.1 Surface Stress Measurements . . . . . . . . . . . . . . . . . 9

2.2.2 Velocity Measurements .................. 10

2.2.3 Temperature Measurements . . . . . . . . . . . . . . 11

2.3 Infrared Thermography for the Analysis of Laminar Separation Bubbles . . 14

2.4 Heat Transfer Fundamentals . . . . . . . . . . . . . . . . . . . 16

3 Experimental Methodology 20

3.1 Experimental Setup . . . . . . . . . . . . . . . . 21

3.1.1 Wind Tunnel Facility . . . . . . . . . . . . . . 21

3.1 .2 Flat Plate Model . . . . . . . . . . . . . . . . . 21

3.1.3 Airfoil Model ..................... 23

3.2 Measurement Techniques . . . . . . . . . . . . . . . . . . . 24

3.2.1 Pressure Measurements . . . . . . . . . . . . . 25

3.2.2 PIV Measurements . . . . . . . . . . . . . . 25 
3.2 .3 Infrared Measurements . . . . . . . . . . . . . . . . . . 27

3.3 Expected Thermal Behaviour of a Laminar Separation Bubble . . . . . . . 30

4 Streamwise Flow Development of a Laminar Separation Bubble on a Flat Plate $\quad 34$

4.1 Baseline Flow Measurements . . . . . . . . . . . . . . . . . . . . . . 34

4.2 Time-Averaged Thermal Analysis . . . . . . . . . . . . . . . . . . . 42

5 Streamwise Flow Development of a Laminar Separation Bubble on a NACA 0018 Airfoil 54

5.1 PIV Measurements . . . . . . . . . . . . . . . . . . . . 55

5.2 Time-Averaged Thermal Analysis . . . . . . . . . . . . . . . . 58

5.3 Transient Heat Transfer Analysis . . . . . . . . . . . . . . . 65

$\begin{array}{llr}6 & \text { Conclusions } & 71\end{array}$

6.1 Laminar Separation Bubble Development On a Flat Plate Geometry . . . . 71

6.2 Laminar Separation Bubble Development On a NACA 0018 Airfoil _ . . . 73

$\begin{array}{lll}7 & \text { Recommendations } & 75\end{array}$

$\begin{array}{ll}\text { References } & 77\end{array}$

$\begin{array}{ll}\text { Appendices } & 88\end{array}$

$\begin{array}{lr}\text { A Supplementary Results } & 89\end{array}$

A.1 Flat Plate Data . . . . . . . . . . . . . . . . . . . . 89

A.2 Airfoil Data . . . . . . . . . . . . . . . . . . 95

B Characterization of the Laminar Separation Bubble with Linear Fitting $\begin{array}{ll}\text { Techniques } & 103\end{array}$

$\begin{array}{ll}\text { C Detailed Energy Balance } & 105\end{array}$

C.1 Flat Plate Analysis . . . . . . . . . . . . . . . . . . . . . . . . 105

C.2 Airfoil Energy Balance . . . . . . . . . . . . . . . . . . . . . . . 110

D Experimental Uncertainty 115

D.1 Uncertainty in Experimental Conditions . . . . . . . . . . . . 115 
D.2 Uncertainty in Pressure Measurements . . . . . . . . . . . . 117

D.3 Uncertainty in PIV Measurements . . . . . . . . . . . . . . . 118

D.4 Uncertainty in Thermal Measurements . . . . . . . . . . . . . . 119 


\section{List of Tables}

3.1 Summary of the flow conditions and corresponding measurements performed on the flat plate geometry . . . . . . . . . . . . . . . . .

3.2 Summary of the flow conditions and corresponding measurements performed on the airfoil geometry . . . . . . . . . . . . . . . . . 21

3.3 Important PIV parameters for flat plate experiments . . . . . . . . . . 27

3.4 Important PIV parameters for airfoil experiments . . . . . . . . . . . . 28

3.5 Important infrared thermography parameters for each experimental investi-

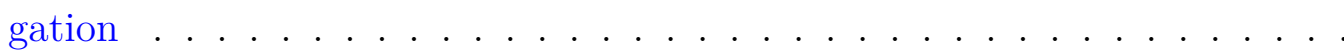

4.1 Estimated locations of mean separation, transition, and reattachment from pressure and PIV measurements. . . . . . . . . . . . . . .

4.2 Estimated locations of mean separation, transition, and reattachment on the flat plate from each measurement technique. . . . . . . . . . . . 53

5.1 Estimated characteristic locations based on PIV measurements . . . . . . . 56

5.2 Estimated locations of mean separation, transition, and reattachment from PIV and temperature-based measurements on the airfoil. . . . . . . . . . . 65

C.1 Summary of variables, and their respective values, used in the full energy balance on the flat plate model . . . . . . . . . . . . . . 108

C.2 Summary of variables, and their respective values, used in the full energy balance on the airfoil model . . . . . . . . . . . . . . . . . . 113

D.1 Summary of uncertainty estimates for flat plate experiments . . . . . . 116

D.2 Summary of uncertainty estimates for airfoil experiments . . . . . . . 117 


\section{List of Figures}

2.1 (a) Separated shear layer failing to reattach, and (b) forming a laminar separation bubble, after Yarusevych et al. [32] and Kurelek [53] . . . . . .

2.2 Topology of a laminar separation bubble, after Horton [4] and Kirk \& Yarusevych [54] . . . . . . . . . . . . . . . . . . . . .

2.3 Methodology presented by Raffel \& Merz [21] for detecting the transition region for a pitching airfoil. Adapted from Raffel \& Merz [21] . . . . . . .

2.4 Development of a laminar boundary layer on a flat plate with zero pressure gradient undergoing natural transition to turbulent flow with corresponding distributions of convective heat transfer coefficient and surface temperature

3.1 Recirculating wind tunnel at the University of Waterloo . . . . . . . . . . . 22

3.2 (a) Side, and (b) top view of the flat plate model . . . . . . . . . . . 23

3.3 (a) Side, (b) isometric, and (c) section view of the airfoil model . . . . . . 24

3.4 PIV fields of view and corresponding correlation algorithms used for (a) flat plate, and (b) airfoil experiments . . . . . . . . . . . . . . . 27

3.5 Configuration of experimental equipment for the (a) flat plate, and (b) airfoil experiments. . . . . . . . . . . . . . . . .

3.6 Pressure gradient and boundary layer characteristics in a laminar separation

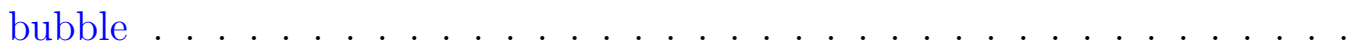

3.7 (a) Formation of thermal and velocity boundary layers on a flat plate subject to a constant heat flux, and (b) corresponding wall temperature development along the streamwise direction . . . . . . . . . . . . . . . . . . . 32

4.1 Methodology employed by Boutilier \& Yarusevych [7] for estimation of the characteristic points in a laminar separation bubble using static pressure distributions shown with an example pressure distribution $\left(\operatorname{Re}_{L}=27,000\right) \quad 35$

4.2 Comparison of inviscid and measured pressure distributions at $\operatorname{Re}_{L}=27,00036$

4.3 Distribution of static pressure coefficient for highlighted flow conditions . . 37 
4.4 Contours of mean streamwise velocity at highlighted Reynolds numbers . . 38

4.5 Shape factor distribution and corresponding mean transition estimates . . 39

4.6 Contours of the streamwise RMS component of fluctuating velocity . . . . 41

4.7 Contours of the wall-normal RMS component of fluctuating velocity . . . . 42

4.8 Reynolds shear stress contours . . . . . . . . . . . . . . . . 43

4.9 Instantaneous contours of spanwise vorticity at $\operatorname{Re}_{L}=36,000 \quad \ldots$. . . . 44

4.10 Contours of time-averaged temperature measured on the flat plate surface . 46

4.11 Time- and spanwise-averaged distributions of surface temperature and its streamwise gradient . . . . . . . . . . . . . . . . . 47

4.12 Time- and spanwise-averaged distribution of normalized quiescent surface temperature ............................. 49

4.13 Time- and spanwise-averaged distributions of $h^{*}$ and its streamwise gradient 51

5.1 Contours of time-averaged streamwise velocity at each highlighted flow con-

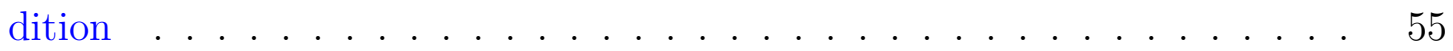

5.2 Shape factor distributions with associated transition estimates . . . . . . 56

5.3 Contours of the streamwise RMS component of fluctuating velocity . . . . 57

5.4 Contours of the wall-normal RMS component of fluctuating velocity . . . . 58

5.5 Reynolds shear stress contours . . . . . . . . . . . . . . . . 59

5.6 Contours of instantaneous spanwise vorticity at $\mathrm{Re}_{c}=120,000$. . . . . . 60

5.7 Contours of time-averaged temperature measured on the airfoil surface . . 61

5.8 Time- and spanwise-averaged distributions of surface temperature and its streamwise gradient . . . . . . . . . . . . . . . . . . . 62

5.9 Time- and spanwise-averaged distribution of normalized surface temperature in quiescent conditions . . . . . . . . . . . . . . . . 63

5.10 Time- and spanwise-averaged distributions of $h^{*}$ and its streamwise gradient 64

5.11 Transient development of surface temperature resulting from a step change in radiative heating at $x \approx 0.5 c$ and $\mathrm{Re}_{c}=120,000 \ldots \ldots$. . . . .

5.12 Distribution of convective heat transfer coefficient calculated from transient thermal decline plotted on an arbitrary scale . . . . . . . . . . 68

5.13 Spanwise-averaged distribution of normalized convective heat transfer coefficient and its streamwise gradient calculated with a transient time of $6 \mathrm{~s}$.

A.1 Measured surface pressure distribution on the flat plate at each investigated flow condition . . . . . . . . . . . . . . . . . 90 
A.2 Linearity shown between maximum displacement thickness and total separation bubble length measured with PIV in flat plate experiments . . . . .

A.3 Transient surface temperature development with the onset of radiative heating in quiescent conditions shown at specified streamwise locations on the flat plate . . . . . . . . . . . . . . . . . . . . . . .

A.4 Time- and spanwise-averaged distributions of (a) surface temperature, and (b) streamwise temperature gradient for each investigated experimental condition on the flat plate . . . . . . . . . . . . . . . . . . . .

A.5 Time- and spanwise-averaged distributions of (a) $h^{*}$, and (b) its streamwise gradient for each investigated experimental condition on the flat plate . . .

A.6 Locations of mean separation, transition, and reattachment estimated from distributions of surface pressure, surface temperature, and $h^{*}$, at each flat plate flow condition . . . . . . . . . . . . . . . . .

A.7 Transient surface temperature development with the onset of radiative heating in quiescent conditions shown at specified streamwise locations on the

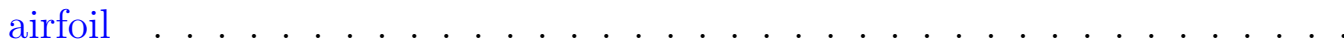

A.8 Time- and spanwise-averaged distributions of (a) surface temperature, and (b) streamwise temperature gradient for each investigated Reynolds number at $\alpha=4^{\circ} \ldots \ldots \ldots \ldots \ldots \ldots \ldots$

A.9 Time- and spanwise-averaged distributions of (a) surface temperature at $\operatorname{Re}_{c}=80,000$, (b) surface temperature at $\operatorname{Re}_{c}=120,000$, (c) streamwise temperature gradient at $\operatorname{Re}_{c}=80,000$, and (d) streamwise temperature gradient at $\mathrm{Re}_{c}=120,000$, at each investigated angle of attack . . . . . . .

A.10 Time- and spanwise-averaged distributions of (a) $h^{*}$, and (b) its streamwise gradient, for each investigated Reynolds number at $\alpha=4^{\circ}$. . . . . . .

A.11 Time- and spanwise-averaged distributions of (a) $h^{*}$ at $\operatorname{Re}_{c}=80,000$, (b) $h^{*}$ at $\operatorname{Re}_{c}=120,000$, (c) streamwise $h^{*}$ gradient at $\operatorname{Re}_{c}=80,000$, and (d) streamwise $h^{*}$ gradient at $\operatorname{Re}_{c}=120,000$, at each investigated angle of attack100

A.12 Locations of mean separation, transition, and reattachment estimated from each measurement technique at $\alpha=4^{\circ}$ and each investigated Reynolds number 101

A.13 Locations of mean separation, transition, and reattachment estimated from each measurement technique at (a) $\operatorname{Re}_{c}=80,000$, and (b) $\operatorname{Re}_{c}=120,000$ and each investigated angle of attack. Characteristic locations estimated from surface pressure measurements are included from Gerakopulos et al. [8] 102 
B.1 Methodology employed by Boutilier \& Yarusevych [7] for estimation of the characteristic locations in a laminar separation bubble using the static pressure distribution at each highlighted Reynolds number . . . . . . . . . . . 104

B.2 Methodology used for detection of mean separation using distributions of $h^{*}$ on the flat plate at highlighted Reynolds numbers . . . . . . . . . . . . .

C.1 Thermal diagram showing (a) the coordinate system and boundary conditions applied for the numerical thermal analysis within the flat plate model, and (b) the differential element considered in the energy balance . . . . . .

C.2 Time-averaged temperature distribution within the flat plate model in (a) quiescent, and (b) flow conditions $\left(\mathrm{Re}_{L}=27,000\right)$ determined with numerical analysis . . . . . . . . . . . . . . . . . . . . . .

C.3 Contribution of each heat transfer mode to the variation of convective heat transfer coefficient in the flat plate energy balance . . . . . . . . . . . 109

C.4 Normalized distributions of convective heat transfer coefficient where the effect of considering radiative cooling and wall-normal heat flux is assessed. Data are presented with corresponding streamwise gradients at $\operatorname{Re}_{L}=27,000110$

C.5 Thermal diagram showing (a) the coordinate system and boundary conditions applied for the numerical thermal analysis within the airfoil model, and (b) the differential element considered in the energy balance . . . . . .

C.6 Time-averaged temperature distribution within the airfoil model in (a) quiescent, and (b) flow conditions $\left(\operatorname{Re}_{c}=80,000\right)$ determined with numerical analysis . . . . . . . . . . . . . . . . . . .

C.7 Contribution of each heat transfer mode to the variation of convective heat transfer coefficient in the airfoil energy balance . . . . . . . . . . . . . . . 114

C.8 Normalized distributions of convective heat transfer coefficient where the effect of considering radiative cooling is assessed. Data are presented with corresponding streamwise gradients at $\operatorname{Re}_{c}=80,000$ and $\alpha=4^{\circ}$. . . . .

D.1 Time- and spanwise-averaged distribution of the streamwise gradient of surface temperature for each highlighted Reynolds number on the flat plate with associated uncertainty bounds . . . . . . . . . . . . . .

D.2 Time- and spanwise-averaged distribution of the streamwise gradient of $h^{*}$ for each highlighted Reynolds number on the flat plate with associated uncertainty bounds ...................... 
D.3 Time- and spanwise-averaged distribution of the streamwise gradient of surface temperature for each highlighted Reynolds number on the airfoil with associated uncertainty bounds . . . . . . . . . . . . . . . 122

D.4 Time- and spanwise-averaged distribution of the streamwise gradient of $h^{*}$ for each highlighted Reynolds number on the airfoil with associated uncertainty bounds . . . . . . . . . . . . . . . . . . . . 123

D.5 Example of the methodology employed for estimating the uncertainty in each characteristic location shown for the separation estimate from surface temperature gradient profiles on the airfoil geometry . . . . . . . . . 123

D.6 Spanwise-averaged distribution of the convective heat transfer coefficient from transient-based measurements with associated uncertainty bounds . .

D.7 Spanwise-averaged distribution of the streamwise gradient of the convective heat transfer coefficient from transient-based measurements with associated uncertainty bounds . . . . . . . . . . . . . . . . . . . . 


\section{Nomenclature}

\section{Dimensionless Quantities}

\begin{tabular}{|c|c|c|}
\hline Symbol & Description & Definition \\
\hline$C_{f}$ & friction coefficient & $\frac{\tau_{w}}{\frac{1}{2} \rho U_{0}^{2}}$ \\
\hline$C_{P}$ & pressure coefficient & $\frac{P-P_{\infty}}{\frac{1}{2} \rho U_{0}^{2}}$ \\
\hline$H$ & shape factor & $\frac{\delta^{*}}{\theta}$ \\
\hline$h^{*}$ & $\begin{array}{l}\text { estimated distribution of normalized forced con- } \\
\text { vective heat transfer coefficient }\end{array}$ & $\frac{h}{h_{\text {Free }}}$ \\
\hline$h_{\mathrm{Min}}^{*}$ & $\begin{array}{l}\text { minimum estimated streamwise value of normal- } \\
\text { ized forced convective heat transfer coefficient }\end{array}$ & $\left.\frac{h}{h_{\text {Free }}}\right|_{\text {Min }}$ \\
\hline $\mathrm{Nu}_{\mathrm{x}}$ & local Nusselt number & $\frac{h x}{k}$ \\
\hline $\operatorname{Pr}$ & Prandtl number & $\frac{\nu}{\kappa}$ \\
\hline $\operatorname{Re}_{c}$ & chord-based Reynolds number & $\frac{U_{0} c}{\nu}$ \\
\hline $\operatorname{Re}_{\delta^{*}}$ & displacement thickness-based Reynolds number & $\frac{U_{0} \delta^{*}}{\nu}$ \\
\hline $\operatorname{Re}_{L}$ & fence height-based Reynolds number & $\frac{U_{0} L}{\nu}$ \\
\hline $\operatorname{Re}_{x}$ & local Reynolds number & $\frac{U_{0} x}{\nu}$ \\
\hline St & Stanton number & $\frac{h}{\rho U_{e} c_{p}}$ \\
\hline
\end{tabular}




\section{Roman Symbols}

\section{Symbol Description}

A

c

$c_{p}$

$c_{p, s}$

$\Delta x, \Delta y, \Delta z$

$h$

$h_{B}$

$h_{B, Q}$

$h_{\text {Free }}$

$h_{\text {Min }}$

$k$

K

$k_{s}$

l

$L$

$P$

$P_{\infty}$

$q_{\text {Cond }}$

$q_{\text {Conv }}^{\prime \prime}$

$q_{\text {Conv }}$

$q_{\text {Rad }}^{\prime \prime}$

area

chord length

surface

fence height thermal heat capacity of fluid

thermal heat capacity of surface material

differential streamwise, wall-normal, and spanwise coordinates

forced convective heat transfer coefficient

convective heat transfer coefficient at the bottom

free convective heat transfer coefficient at the

bottom surface

free convective heat transfer coefficient

minimum streamwise forced convective heat

transfer coefficient

thermal conductivity of fluid

dimensional constant, $K=A / \rho_{s} c_{p, s} V$

thermal conductivity of surface material

extent of separation bubble

static surface pressure

free-stream static pressure

conductive heat flux

convective heat flux per unit area

convective heat flux

radiative heat flux per unit area

\section{Units}

$\mathrm{m}^{2}$

m

$\mathrm{J} \mathrm{kg}^{-1} \mathrm{~K}^{-1}$

$\mathrm{J} \mathrm{kg}^{-1} \mathrm{~K}^{-1}$

m

$\mathrm{W} \mathrm{m}^{-2}$

$\mathrm{W} \mathrm{m}^{-2}$

$\mathrm{W} \mathrm{m}^{-2}$

$\mathrm{W} \mathrm{m}^{-2}$

$\mathrm{W} \mathrm{m}^{-2}$

$\mathrm{W} \mathrm{m}{ }^{-1} \mathrm{~K}^{-1}$

$\mathrm{m}^{2} \mathrm{~J}^{-1}$

$\mathrm{W} \mathrm{m}{ }^{-1} \mathrm{~K}^{-1}$

m

m

$\mathrm{Pa}$

$\mathrm{Pa}$

W

$\mathrm{W} \mathrm{m}^{-2}$

W

$\mathrm{W} \mathrm{m}{ }^{-2}$ 


\begin{tabular}{|c|c|c|}
\hline$q_{\text {Rad }}$ & radiative heat flux & $\mathrm{W}$ \\
\hline$q_{\text {Wall-Normal }}$ & wall-normal heat flux & $\mathrm{W}$ \\
\hline$t$ & time & $\mathrm{s}$ \\
\hline$t_{0}$ & initial measurement time & $\mathrm{s}$ \\
\hline $\bar{T}$ & time-averaged measured surface temperature & ${ }^{\circ} \mathrm{C}$ \\
\hline$T_{0}$ & surface temperature measured at time $t=t_{0}$ & ${ }^{\circ} \mathrm{C}$ \\
\hline$T_{\infty}$ & free-stream temperature & ${ }^{\circ} \mathrm{C}$ \\
\hline$T_{f}$ & film temperature & ${ }^{\circ} \mathrm{C}$ \\
\hline$T$ & temperature & ${ }^{\circ} \mathrm{C}$ \\
\hline $\bar{T}_{Q}$ & $\begin{array}{l}\text { time-averaged surface temperature measured in } \\
\text { quiescent conditions }\end{array}$ & ${ }^{\circ} \mathrm{C}$ \\
\hline$T_{Q}$ & $\begin{array}{l}\text { surface temperature measured in quiescent condi- } \\
\text { tions }\end{array}$ & ${ }^{\circ} \mathrm{C}$ \\
\hline $\bar{T}_{Q, \mathrm{Max}}$ & $\begin{array}{l}\text { maximum time-averaged surface temperature } \\
\text { measured in quiescent conditions }\end{array}$ & ${ }^{\circ} \mathrm{C}$ \\
\hline$T_{w}$ & wall temperature & ${ }^{\circ} \mathrm{C}$ \\
\hline$u, v$ & streamwise and wall-normal velocity components & $\mathrm{ms}^{-1}$ \\
\hline$u^{\prime}, v^{\prime}$ & $\begin{array}{l}\text { fluctuating components of streamwise and wall- } \\
\text { normal velocity }\end{array}$ & $\mathrm{ms}^{-1}$ \\
\hline $\bar{u}_{\mathrm{rms}}^{\prime},{\overline{v^{\prime}}}_{\mathrm{rms}}$ & $\begin{array}{l}\text { root-mean-square of streamwise and wall-normal } \\
\text { fluctuating velocities }\end{array}$ & $\mathrm{ms}^{-1}$ \\
\hline$U_{e}$ & boundary layer edge velocity & $\mathrm{ms} \mathrm{s}^{-1}$ \\
\hline$U_{0}$ & free-stream velocity & $\mathrm{ms}^{-1}$ \\
\hline$V$ & volume & $\mathrm{m}^{3}$ \\
\hline$x, y, z$ & $\begin{array}{l}\text { streamwise, wall-normal, and spanwise coordi- } \\
\text { nates }\end{array}$ & $\mathrm{m}$ \\
\hline$x_{0}$ & initial streamwise value in numerical domain & $\mathrm{m}$ \\
\hline
\end{tabular}


$x_{S}, x_{T}, x_{R} \quad$ streamwise location of mean separation, transition, and reattachment

$X, Y \quad$ streamwise and wall-normal edges of numerical m domain 


\section{Greek Symbols}

\begin{tabular}{|c|c|c|}
\hline Symbol & Description & Units \\
\hline$\alpha$ & angle of attack & $\circ$ \\
\hline$\delta$ & boundary layer thickness & $\mathrm{m}$ \\
\hline$\delta^{*}$ & displacement thickness & $\mathrm{m}$ \\
\hline$\delta_{T}$ & thermal boundary layer thickness & $\mathrm{m}$ \\
\hline$\varepsilon$ & surface emissivity & - \\
\hline$\kappa$ & thermal diffusivity of fluid & $\mathrm{m}^{2} \mathrm{~s}^{-1}$ \\
\hline$\Lambda$ & rate of temperature decline & ${ }^{\circ} \mathrm{Cs}^{-2}$ \\
\hline$\nu$ & kinematic viscosity & $\mathrm{m}^{2} \mathrm{~s}^{-1}$ \\
\hline$\theta$ & momentum thickness & $\mathrm{m}$ \\
\hline$\rho$ & density of fluid & $\mathrm{kg} \mathrm{m}^{-3}$ \\
\hline$\rho_{s}$ & density of surface material & $\mathrm{kg} \mathrm{m}^{-3}$ \\
\hline$\sigma$ & Stefan-Boltzmann constant & $\mathrm{W} \mathrm{m}^{-2} \mathrm{~K}^{-4}$ \\
\hline$\tau_{w}$ & wall shear stress & $\mathrm{N} \mathrm{m}^{-2}$ \\
\hline$\omega$ & spanwise vorticity & $\mathrm{s}^{-1}$ \\
\hline
\end{tabular}




\section{Chapter 1}

\section{Introduction}

In modern day aviation, the airfoil geometry has been applied across an immense range of flow conditions, spanning chord-based Reynolds numbers of $\operatorname{Re}_{c} \approx 10^{3}-10^{9}$ [1]. Over the last century, engineers have astoundingly designed countless airfoil geometries suitable for application across this $\operatorname{Re}_{c}$ range. Lower Reynolds numbers are often associated with decreased aerodynamic performance, particularly for $\operatorname{Re}_{c} \approx 50,000-500,000$ [1,2]. This can be attributed to the extended distance over which the boundary layer remains in a laminar state, resulting in a flow which is vulnerable to boundary layer separation.

Flow passing over the suction surface of an airfoil in the low Reynolds number regime often remain laminar past the point of minimum pressure. The adverse pressure gradient opposes the oncoming flow, which can result in separation of the attached boundary layer and the formation of a separated shear layer. Under certain flow conditions, the separated shear layer can undergo transition and reattach to the airfoil surface in the mean sense, resulting in a region of recirculating fluid bound between the locations of mean separation and reattachment $[1,3,4]$. Such a phenomenon is often referred to as a laminar separation bubble and will be the flow under investigation in this thesis. Laminar separation bubbles affect a number of low-speed flow applications such as unmanned aerial vehicles, small-tomedium scale wind turbines, and glider aircrafts [1]. These applications often experience a reduction in aerodynamic performance, in terms of lift-to-drag ratio, in the presence of laminar separation bubbles $[1,3]$.

Due to the loss of aerodynamic performance associated with the formation of laminar separation bubbles, it is important to properly identify and characterize their existence for a range of flow conditions. Early studies of laminar separation bubbles distinguished between short and long bubbles, based on their streamwise extent relative to boundary layer parameters [5]. Tani [6] later suggested a more practical approach of characterization, where the effect on the measured surface pressure distribution was evaluated. Short 
bubbles were ones which had a negligible effect on surface pressure, whereas long bubbles significantly diminished peak suction values and introduced a region of pressure plateau beneath the separated region. Since then, laminar separation bubbles are often distinguished based on characteristic locations of mean separation, transition, and reattachment, an approach put forth by Horton [4]. Following this characterization, generalized trends of laminar separation bubbles formed on airfoil geometries have been established. For flow conditions which produce laminar separation bubbles, increases in Reynolds number or angle of attack have been shown to advance the bubble location upstream, while reducing the total streamwise extent. Conversely, a reduction of the Reynolds number or angle of attack at similar flow conditions results in a downstream movement of the separated region along with an increase of the total streamwise extent [7,8]. A lower limit exists for both Reynolds number and angle of attack, below which formation of a laminar separation bubble no longer occurs.

Typical identification of mean separation, transition, and reattachment locations provides information regarding the location and extent of the separation bubble, as well as some insight into the transition process [4]. Traditional methods for experimental characterization commonly rely on near-wall velocity measurements, using techniques such as particle image velocimetry (PIV) [9-11] and hot wire anemometry [12,13]. In addition, surface stress estimations from surface pressure taps and hot film sensors have been employed $[7,14]$. Although these methods have been shown to effectively characterize separated flows, their implementation typically involves substantial overhead costs in terms of measurement equipment and/or model instrumentation, as well as experimental setup run times. As a result of the aforementioned drawbacks, recently there has been an increased interest in the development of a non-intrusive measurement technique for detecting and characterizing separating and separating-reattaching flows in a more time-efficient manner. In this regard, Infrared Thermography (IRT) has shown some promise, as the near surface flow development in separating-reattaching flows is characterized by significant changes in the convective heat transfer coefficient. For example, with a surface heated to a temperature higher than the ambient air, such changes in local convective heat transfer result in distinct surface temperature variations that can be related to the underlying flow development [15].

Thomann \& Frisk [16] were amongst the first to apply infrared thermography to study aerodynamic phenomena. They used an infrared camera to analyze flow development over a paraboloid model in Mach 7 flow. Infrared thermography was shown to rapidly provide information regarding near-wall convective heat transfer, with an accuracy comparable to temperature sensitive paints at the time. However, infrared thermography was shown to be the superior technique as it did not require re-application of a surface coating between runs. Since this pioneering work, numerous studies in fluid mechanics have employed infrared 
thermography, with the majority of these studies focused on supersonic flows due to higher convective heat transfer intensity [15]. Such studies have been able to detect phenomena such as laminar-to-turbulent transition [17], and separation [18].

With recent developments in infrared camera technology, infrared thermography has been successfully applied in subsonic flows to identify boundary layer transition in both laboratory [19-25] and in-flight experiments [20,26]. Additionally, analysis of separating near-wall flows has been pursued, such as detection and characterization of laminar separation bubbles. Montelpare \& Ricci [27] used infrared thermography and surface pressure measurements to characterize laminar separation bubbles on an internally-heated Eppler387 airfoil. Although quantitative analysis was not provided, a band of elevated surface temperature was observed on the airfoil surface, which was shown to approximately coincide with the location of the separated region identified in measured distributions of surface pressure. In a follow-up study, Ricci \& Montelpare [28] employed similar techniques to provide a more quantitative analysis of the separated region. A differential energy balance was performed on the airfoil surface, yielding estimated distributions of non-dimensional convective heat transfer coefficient beneath the laminar separation bubble. It was suggested that the point of mean separation can be located at the inflection point in the streamwise distribution of non-dimensional convective heat transfer coefficient, upstream of the absolute minimum value. Additionally, it was suggested that locations of mean transition and reattachment could be identified where the convective heat transfer coefficient achieved a minimum and maximum value, respectively. 


\section{$1.1 \quad$ Study Objectives}

While useful developments towards the characterization of laminar separation bubbles using infrared thermography have been made in previous investigations, there is a need for a reliable comparative analysis of the results with near-wall velocity measurements, which currently represent the most direct diagnostic tool for separating-reattaching flows in experimental studies. A direct comparison of infrared thermography and near-wall velocity measurements can also be used to develop and validate methodologies for estimating mean separation, transition, and reattachment locations from surface temperature distributions. In this regard, the main objectives of this study can be summarized as follows:

1. Establish a set of representative baseline laminar separation bubbles and characterize them experimentally

2. Develop and validate methodologies for estimating the locations of mean separation, transition, and reattachment from infrared thermography-based measurements

3. Compare the established methodologies of infrared-based laminar separation bubble identification and provide recommendations for future implementations 


\section{Chapter 2}

\section{Background}

Flows over airfoils in the low Reynolds number regime have received considerable attention over recent decades due to their vulnerability to boundary layer separation. Since applications such as lightweight aircrafts, small to medium scale wind turbines, and unmanned aerial vehicles (UAVs) primarily operate in this regime, the analysis and detection of the separated flow region is of high importance $[1,2]$. The focus of this chapter is to review the results of previous studies pertaining to separated low Reynolds number flows and the associated diagnostic techniques.

\subsection{Laminar Separation Bubbles at Low Reynolds Num- bers}

Low Reynolds number flow over an airfoil is typically characterized with Reynolds numbers, based on the airfoil chord length, between 50,000 and 500,000 [1,2]. Boundary layer separation can occur in this regime, negatively affecting the aerodynamic performance in terms of decreased lift, increased drag, unsteady loading, and noise production [1,2, 29]. Therefore, numerous studies have focused on this regime, with significant contributions made both experimentally [30-41] and numerically [31,42-51] on geometries such as airfoils $[30,32-34,38-41,49]$ and flat plates with imposed pressure gradients $[9,12,31,35-$ $37,42,44,46,47,50]$.

As a result of the low Reynolds number, when the flow passes over the suction side of the airfoil surface, it often remains in a laminar state downstream of the point of minimum pressure. Due to the laminar boundary layer having significantly less near-wall streamwise momentum compared to a turbulent boundary layer, the adverse pressure gradient often causes the flow to separate from the surface, forming a separated shear layer $[1,4]$. 


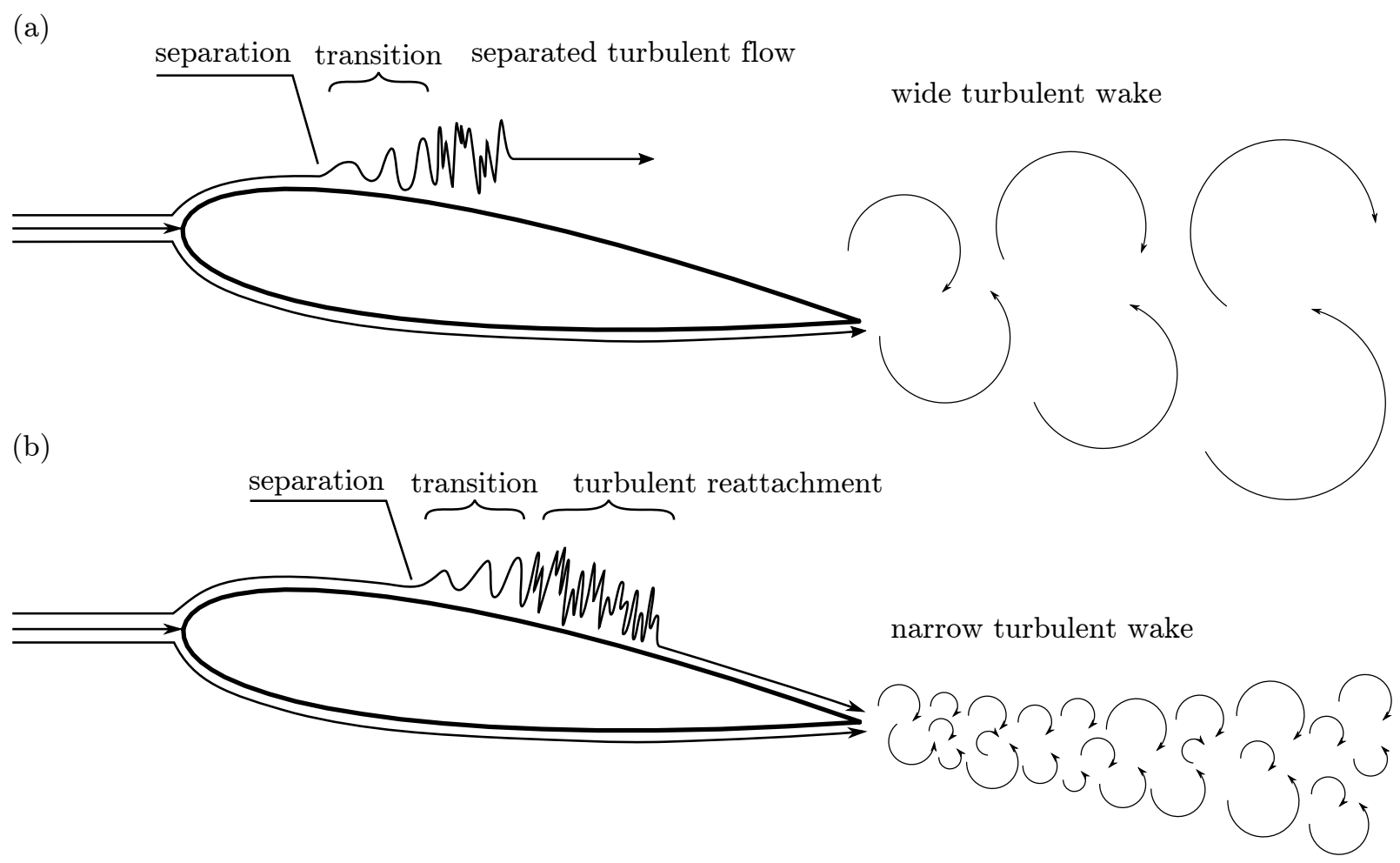

Figure 2.1: (a) Separated shear layer failing to reattach, and (b) forming a laminar separation bubble, after Yarusevych et al. [32] and Kurelek [53].

Depending on flow conditions, this separation can result in two different outcomes, as shown in Fig. 2.1. If the Reynolds number is below some threshold value for a given angle of attack, the separated shear layer will fail to reattach before the trailing edge [52] (Fig. 2.1a), leaving the airfoil in a stalled state. This scenario will significantly reduce the aerodynamic performance by decreasing the lift and increasing the drag. Alternatively, if the Reynolds number is sufficiently high for a given angle of attack, the separated shear layer can transition to turbulence and reattach to the airfoil surface in a turbulent state [3] (Fig. 2.1b). This forms what is known as a laminar separation bubble (LSB) between the points of separation and reattachment [4]. The presence of an LSB is less detrimental to the aerodynamic performance compared to a fully stalled airfoil; however, not to the point as in the absence of separation $[2,3]$.

Although numerous studies have been performed on LSBs, the topology described by Horton [4], as shown in Fig. 2.2, is still widely accepted today. The locus of zero cumulative mass-flux, or mean dividing streamline, intersects the model surface at the points of separation and reattachment. Between these points lies the region where transition to turbulence occurs; however, transition is often ascribed to a point, rather than region [3]. In the wall-normal direction, the region between the outer flow and the dividing streamline 


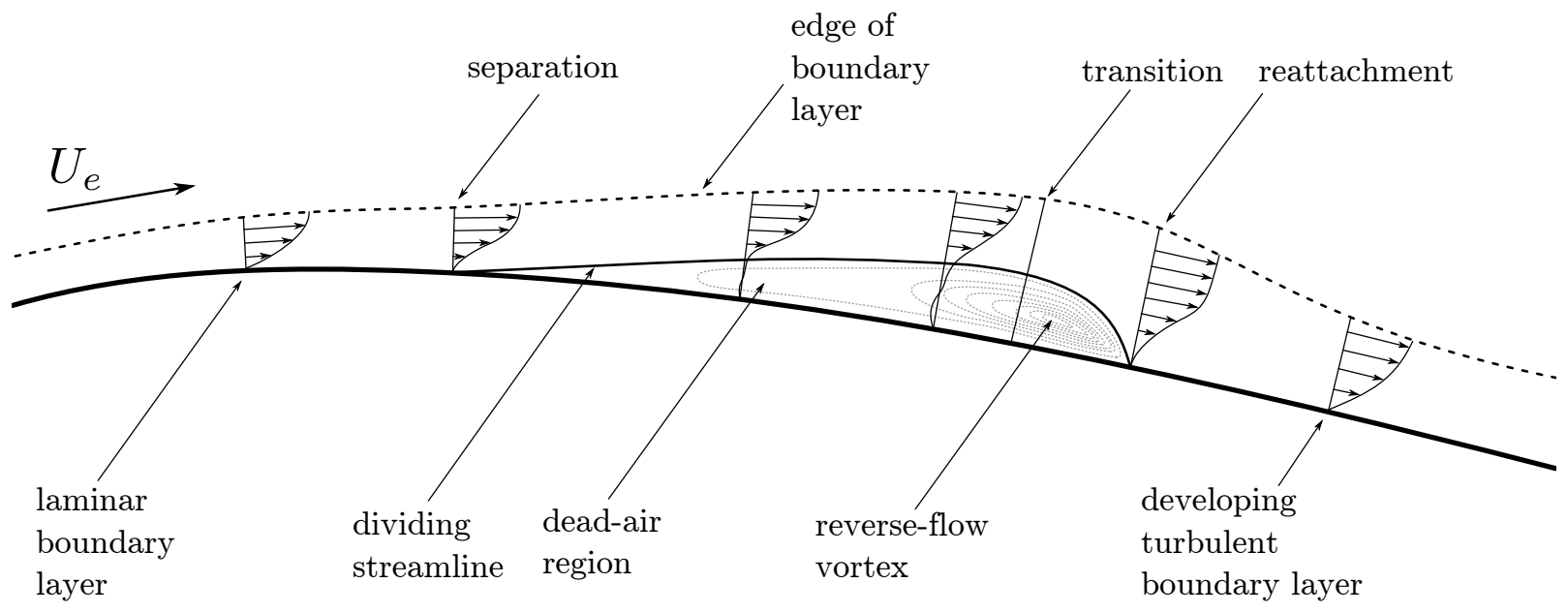

Figure 2.2: Topology of a laminar separation bubble, after Horton [4] and Kirk \& Yarusevych [54].

is referred to as the separated shear layer. The recirculation region is located below the dividing streamline, which exhibits different behaviours upstream and downstream of the mean transition location. Upstream of transition, the recirculating region is characterized by slow-moving or stagnant fluid and is sometimes referred to as the dead-air region. Following transition, in what is sometimes called the reverse-flow vortex, the velocities are much higher due to the roll-up and shedding of vortex structures during the reattachment process [4].

Since the aerodynamic performance of airfoils operating in the low Reynolds number regime is affected by boundary layer separation, it is of utmost importance to determine the presence, location, and extent of the separated region [1]. Early studies of laminar separation bubbles initiated this characterization by distinguishing between short and long separation bubbles, based on the extent of their streamwise footprint in relation to boundary layer parameters. Owen \& Klanfer [5] suggested that the ratio of the bubble length to the displacement thickness at the point of separation could be used as an indicator of overall bubble behaviour. Using this ratio, short bubbles were shown to have $l / \delta^{*}$ on the order of 100 , and long bubbles were shown to have $l / \delta^{*}$ on the order of 10,000 . More specifically, Owen \& Klanfer [5] suggested that short bubbles have a Reynolds number at separation (defined $\operatorname{Re}_{\delta^{*}}=U_{e} \delta^{*} / \nu$ ) greater than, and long bubbles have a Reynolds number less than, a critical value between 400 - 500. Expanding on this, Tani [6] suggested that the effect of the laminar separation bubble on the static pressure distribution was a more preferable method for distinguishing between short and long bubbles, as this was 
related to the aerodynamic performance. Short bubbles are ones which have a negligible effect on the static pressure distribution, specifically the peak suction value. Alternatively, long bubbles are ones which significantly alter the static pressure distribution by diminishing the suction peak and introducing a pressure plateau, spanning several percent of the chord, characterizing the fore portion of the bubble. Since then, the presence of a laminar separation bubble is usually diagnosed based on its characteristic points, namely, the points of separation, transition, and reattachment [4].

The transition process in the separated shear layer has received considerable attention over the last decades as it is still not fully understood [31-33, 36-40,47, 48, 55, 56]. Disturbances in the free-stream, either sound or vorticity fluctuations, perturb the boundary layer through a process called receptivity [57]. With the onset of an instability, which can depend on surface curvature, surface roughness, turbulence intensity, and Reynolds number $[57,58]$, these perturbations can amplify. Initial amplification has been shown to be dominated by a Kelvin-Helmholtz instability and can be well described using linear stability theory $[13,37,59,60]$. The later stages of transition are marked with non-linear and three-dimensional interactions leading to the formation of spanwise-oriented vortices; which are shed from the boundary layer at the fundamental separated shear layer instability frequency $[9,13,32,35,37]$. The exchange of momentum with the outer flow as a result of the roll-up process allows the shear layer to recover the necessary momentum needed to reattach to the surface and develop a turbulent boundary layer $[3,5]$.

Although the separation bubble is receptive to a number of flow-specific parameters, such as free-stream disturbances, trends for estimating the separation bubble extent have been established for both Reynolds number and angle of attack [7, 10,33]. At low angles of attack, provided the Reynolds number is above approximately 50,000, the separation bubble covers a relatively large portion of the airfoil surface. As the angle of attack is increased, the separation and reattachment points shift in the upstream direction, with the latter moving more substantially than the former [7]. The earlier onset of separation can be attributed to the increased strength of the adverse pressure gradient, which results in a more significant deceleration of the near-wall flow. The increased angle of attack results in a higher edge velocity and a thinner boundary layer at the point of separation [13]. These characteristics cause a more pronounced instability in the separated shear layer due to higher wall-normal velocity gradients. The more pronounced instability causes higher rates of disturbance amplification, leading to significant upstream movement of the transition and reattachment locations. Although more subtle, similar trends become evident as the Reynolds number is increased for a fixed angle of attack [7, 10,33, 35, 61]. This results in the upstream movement, and decreased streamwise footprint, of the separation bubble. Conversely, if the Reynolds number is reduced, the separation bubble increases in length 
and moves towards the trailing edge. This trend breaks down if the Reynolds number decreases below a critical value of $\operatorname{Re}_{c} \approx 50,000$, where the separated shear layer suddenly fails to reattach, resulting in a significant reduction in aerodynamic performance due to an abrupt reduction in lift $[1,2,52]$. Significant changes in bubble topology resulting from minor changes in flow condition is a phenomenon known as bubble bursting and is often associated with Gaster [52].

\subsection{Measurement Techniques for Laminar Separation Bubbles}

Since the focus of this thesis is the evaluation of a relatively new measurement technique, it is beneficial to review traditional measurement techniques for separated flows, as they provide baseline data used for comparison and validation. These techniques are categorized as surface stresses measurements in Section 2.2.1, velocity measurements in Section 2.2.2, and surface temperature measurements in Section 2.2.3.

\subsubsection{Surface Stress Measurements}

Surface pressure distributions obtained with measurements of static pressure can be analyzed to infer details about flow behaviour, with a number of studies implementing this technique for the analysis of separated flows [6,7,33,34,62-64]. As a result of the near stagnant fluid in the fore portion of the separation bubble, the point of mean separation can be distinguished as the onset of a pressure plateau in the streamwise direction. Following the pressure plateau, the beginning and end of the rapid pressure recovery region marks the locations of mean transition and reattachment, respectively [6,33]. Measurements of surface pressure fluctuations have also been used to estimate the location and extent of laminar separation bubbles [63,65], while resolving details regarding the transition process such as the most unstable frequency, growth rates of disturbances, and convective velocities of coherent structures $[63,65]$. For both time-averaged and time-resolved measurements of surface pressure, a common downside is the required model modification to instrument it with the necessary equipment. Moreover, once instrumented, the spatial resolution is typically fixed and often limited due to placement of the pressure taps [66].

In order to mitigate the issues with spatial resolution, surface films which respond directly to changes in surface stress, such as pressure sensitive paints, have also been implemented. These paints have luminescent molecules which emit photons of different

wavelengths corresponding to different magnitudes of surface pressure [66]. Although the 
use of pressure sensitive paints has been shown to accurately detect boundary layer phenomena in transonic and supersonic conditions [67], implementation below Mach numbers of $\approx 0.3$ is often difficult due to insufficient sensitivity [68].

Film-based techniques which respond to changes in the magnitude of shear stress however, have been more successful at the lower velocities typical to the low Reynolds regime $[14,62]$. Lee \& Basu [14] used multiple closely-spaced hot-film sensor arrays to analyze laminar separation bubble topology on a pitching NACA 0012 airfoil. They were able to establish trends in the separation bubble extent and location based on changes in the angle of attack. Likewise, Zhong [62] implemented shear-sensitive liquid crystals to characterize laminar separation bubbles on a NACA 66 60012 airfoil. The presence of the laminar separation bubble was confirmed for moderate angles of attack when compared to surface pressure measurements and surface oil-flow visualization patterns. However, at low angles of attack, it was established that the change in magnitude of shear stress was too low and liquid crystals are not suitable for separation detection.

\subsubsection{Velocity Measurements}

Information regarding near-wall velocity within a laminar separation bubble allows for diagnostics of its location, streamwise extent, and detailed analysis of the flow physics. Hot wire anemometry (HWA) offers a high-frequency point measurement of flow velocity by introducing a probe into the region of interest. This method has been implemented in various studies focused on low-Reynolds flows containing laminar separation bubbles $[12,33,35,38,54,69]$. O'Meara \& Mueller [33] used HWA to analyze the wall-normal extent of laminar separation bubbles for a range of Reynolds numbers. They found that the bubble height at the transition location decreased almost linearly with Reynolds number. Moreover, as later supported by Burgmann \& Schröder [10], O'Meara \& Mueller [33] found that the bubble height at transition was nearly linearly related to the total bubble length. Watmuff [12] implemented an automated flying HWA system to analyze the instability of laminar separation bubbles on a flat plate subject to an adverse pressure gradient. He showed that the amplification of disturbances in the separated shear layer was similar to that expected due to a Kelvin-Helmholtz instability. More recently, Kirk \& Yarusevych [54] used HWA to characterize the formation of coherent structures within the separated shear layer. It was shown that although the roll-up process is initially coherent in the spanwise direction, non-uniformities rapidly arise across the span as vortices develop downstream.

Particle image velocimetry (PIV) is frequently used in fluid mechanics and can measure up to all three velocity components in a plane or volume. Many researchers have used this technique to analyze laminar separation bubbles forming on airfoils [10, 39, 55, 56, 70-73] and flat plates with imposed pressure gradients $[9,31,37,74]$. Planar PIV is the most 
common implementation, as it is the simplest configuration and still allows for detailed investigations of flow behaviour. Planar two-component PIV has allowed for analysis of the transition and reattachment process [31, 55, 71], response to periodic impulses [56, 73, 74], along with serving as a reference measurement for numerical studies [37,39]. A timeresolved, stereo-scanning, planar PIV configuration was implemented by Burgmann \& Schröder [10] which allowed for a three-dimensional reconstruction of the flow field within the laminar separation bubble on a SD7003 airfoil. This technique permitted the visualization of vortex structures and their deformation in the transition process. Additionally, trends in the LSB topology arising from changes in Reynolds number and angle of attack were found to agree with previous studies [33]. More recently, Michelis et al. [9] used a combination of planar and tomographic PIV to analyze the bubble topology and threedimensional characteristics of the reattachment process, respectively. They were able to observe non-uniform spanwise development of coherent structures in the separated shear layer, and how impulsive forcing could improve the uniformity of these structures in the spanwise direction.

Although widely accepted, hot wire anemometry and particle image velocimetry both have their limitations when applied in separated flows. Hot wire anemometry is unable to resolve flow direction and suffers from poor signal-to-noise ratio when measuring the low, near-wall velocities $[12,13]$. Furthermore, the physical intrusiveness of placing a probe within the separated region has been shown to alter the topology of the flow $[61,69,75]$. Similarly, the low, near-wall velocities also pose a challenge for PIV, especially if a wide range of velocities are captured in a single field of view. Moreover, PIV equipment often comes with a significant overhead cost, and performing a successful PIV campaign can be time consuming compared to other methods. Therefore, there is an interest in further development of thermal measurement techniques such as the ones described in Section 2.2.3.

\subsubsection{Temperature Measurements}

Measurements of surface temperature on aerodynamic bodies allow for an indirect method of uncovering details of the near-wall flow. A common technique for performing such a measurement is the use of temperature sensitive paints (TSPs), which work much in the same way as pressure sensitive paints [66]. When luminescent molecules within the paint are excited by an external light source, incident photons are absorbed while photons of specific wavelengths are released, with wavelengths that depend on the local surface temperature [66,67]. Due to relatively large temperature magnitudes and gradients, TSPs were initially implemented in hypersonic flows [66,76-78], allowing for the formation of qualitative [77] and quantitative [76] relationships between surface temperature and heat transfer. By increasing thermal contrast with an external heating source, TSPs have also 
been implemented to study near-wall behaviour in the subsonic regime [68, 79].

Following an approach outlined by Liu \& Woodiga [80], Miozzi et al. [81] used TSPs to analyze the topology and coherent structures within an LSB formed on a NACA 0015 hydrofoil. This analysis was further developed by Miozzi et al. [79] who tracked the thermal signatures of near-wall disturbances in high-speed image sequences. A cross-correlation technique was used to determine the time, $\Delta t$, elapsed for the thermal signature of a disturbance to travel a distance of $\Delta x$. From these values, the propagation speed of near-wall thermal disturbances was calculated and related to the local skin friction. Comparison of the skin-friction distribution and mean temperature profiles suggested that the separation and reattachment locations correspond approximately to locations of local maximum and minimum surface temperature, respectively. Furthermore, as suggested by other studies $[19,20,24,82-85]$, the transition location was shown to coincide with the location of maximum streamwise gradient in surface temperature.

The final method discussed, and the focus of this thesis, is infrared thermography (IRT). Infrared thermography is a technique based on the principle that every surface emits electro-magnetic radiation, in the infrared spectrum, which is related to the local surface temperature [86]. Using an infrared camera, local measurements of this electromagnetic radiation are acquired and used to estimate the surface temperature distribution [86]. Thomann \& Frisk [16] were one of the initial groups to apply this technology to the field of aerodynamics. Hypersonic flow around a paraboloid shape was analyzed in order to evaluate the feasibility of applying infrared thermography to study heat transfer in complex flows. They found that infrared thermography was a viable method for obtaining a quick survey of the surface temperature, which could be related to heat transfer, over complex shapes. The accuracy associated with the measurement of surface temperature was shown to be comparable to that of temperature sensitive paints at the time. Moreover, no preparation of the model surface was required between runs, making infrared thermography faster and less labour intensive compared to temperature sensitive paints.

Similar to temperature sensitive paints, initially infrared thermography was primarily implemented in supersonic and hypersonic flows due to the relatively low sensitivity of infrared cameras at the time. Infrared thermography was shown to be capable of detecting aerodynamic phenomena such as laminar-to-turbulent transition $[87,88]$ and the formation of shock waves $[18,88]$. In the subsonic regime, less pronounced temperature differences between the outer flow and the model surface made diagnostics using infrared thermography more difficult. In attempts to improve thermal contrast in the subsonic regime, external $[21,22,25,26,89-91]$ or internal $[19,20,24,90]$ heating elements were often incorporated [82]. With the use of additional heating, both laboratory [19-25] and in-flight experiments $[20,26]$ have performed successful detection of mean transition using infrared 


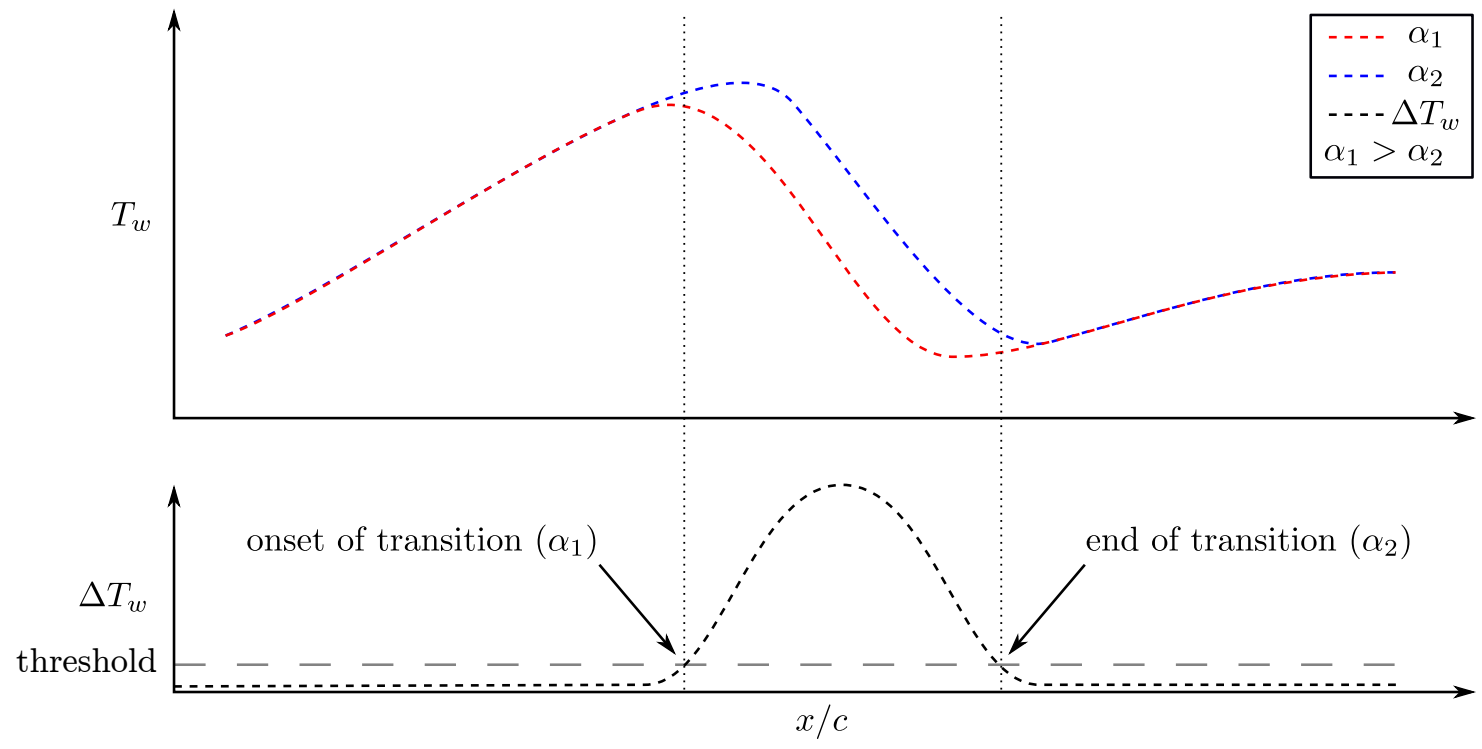

Figure 2.3: Methodology presented by Raffel \& Merz [21] for detecting the transition region for a pitching airfoil. Adapted from Raffel \& Merz [21].

thermography.

Although it is generally agreed upon that infrared thermography can be used for detection of laminar-to-turbulent transition in the sub-sonic regime, different methodologies for doing so have been suggested. A common methodology implemented for detection of laminar-to-turbulent transition is the analysis of streamwise temperature gradients [19,20,24,82-85]. As further explained in Section 2.4, streamwise gradients of surface temperature can be used to identify the transition location as laminar regions experience significantly less convective heat transfer compared to turbulent ones. Therefore, as the flow transitions, the surface will experience relatively large streamwise gradients of convective heat transfer. This results in relatively large gradients in surface temperature, where the streamwise location of absolute maximum temperature gradient coincides with the mean transition location. This methodology was modified by von Hoesslin et al. [25] where the convection-dominant temperature decline of an airfoil subject to a finite heat pulse was recorded. By relating the rate of temperature decline, $\Lambda$, to the convective heat transfer coefficient, von Hoesslin et al. [25] showed that the transition location could be estimated in the region where the streamwise gradient of $\Lambda$ was maximized, as $\Lambda$ was shown to be approximately proportional to the convective heat transfer coefficient.

Raffel \& Merz [21] established a methodology which allows for laminar-to-turbulent transition detection in unsteady flow conditions, such as those experienced by pitching airfoils. By analyzing the distribution of temperature difference between two subsequent high-speed infrared images on a pitching airfoil, the locations corresponding to the begin- 
ning and end of transition can be estimated. Assuming the change in angle of attack is small between the two images, the temperature distributions before and after the transition region are nearly identical. Therefore, the image corresponding to the higher angle of attack, $\alpha_{1}$, will transition first, resulting in a large increase in temperature difference between the two images. The temperature difference will achieve a maximum value as the flow at a lower angle of attack, $\alpha_{2}$, transitions, before the temperature difference returns to zero once the $\alpha_{2}$ flow has achieved a turbulent state. Therefore, Raffel \& Merz [21] defines the onset of transition at $\alpha_{1}$ as the location where the temperature difference first deviates by a threshold value from zero. Likewise, the end of the transition region at $\alpha_{2}$ is defined as the location where the temperature difference returns to a threshold value close to zero. This is shown schematically in Fig. 2.3. Although the methodology suggested by Raffel \& Merz [21] was shown to agree with numerical simulations, Richter et al. [91] and Gardner et al. [92] showed the temperature difference between two thermal images more accurately determines the mean transition location of the flow condition that is an average of the flow conditions in the thermal images (i.e. $\left.\left(\alpha_{1}+\alpha_{2}\right) / 2\right)$. This conclusion was made after comparing the transition locations estimated with surface hot-film sensors, static pressure distributions, and numerical simulations.

\subsection{Infrared Thermography for the Analysis of Lam- inar Separation Bubbles}

With the development of infrared technology, improvements to the thermal sensitivity of modern infrared cameras has allowed for more precise analysis of aerodynamic phenomena, such as the detection of characteristic points in laminar separation bubbles. De Luca et al. [19] used a scanning infrared radiometer to measure the temperature distribution on a Gottingen-797 airfoil experiencing the following: i) transition in the attached boundary layer, ii) fully stalled flow, and iii) a laminar separation bubble. A boundary layer trip was used to disturb the laminar boundary layer and initiate transition for the attached flow. Agreeing with Quast [82], the location of transition was shown to coincide with the location of maximum streamwise temperature gradient, distinguished with closely-spaced isothermal bands in the temperature distribution. Additionally, the stalled flow was shown to result in higher surface temperature than the attached flow due to lower near-wall velocities. For the scenario corresponding to the formation of a laminar separation bubble, estimations of the convective heat transfer coefficient were obtained through an energy balance of a thin metallic foil coating on the airfoil surface. The thin film was supplied a known heat flux through Joule heating, which was balanced by forced convection provided by the flowing air. Losses due to radiation were neglected as the film was heated 
only slightly above the ambient temperature. Conduction to the airfoil model was also neglected as the airfoil model itself was manufactured out of a thin layer of low thermal conductivity material, backed with a thermal insulator. This energy balance allowed for the streamwise distribution of Stanton number to be obtained. Although comparison to another measurement technique was not performed, it was stated that the point of separation coincides with the location of minimum Stanton number as this location experiences minimal convective cooling. Conversely, the reattachment location was stated to coincide with the absolute maximum in the Stanton number distribution, corresponding to the location of maximum convective heat transfer intensity.

Montelpare \& Ricci [27] studied the thermal signatures on an Eppler-387 airfoil in the presence of a laminar separation bubble. In this study, the model was internally heated and infrared images were compared to surface pressure distributions at Reynolds numbers of 100,000 and 200,000 between angles of attack of $-4^{\circ}$ and $14^{\circ}$. The results showed a spanwise band of increased surface temperature for both Reynolds numbers at pre-stall angles of attack. Although specific characterization of the laminar separation bubble was not performed, when compared to the distribution of surface pressure it was shown that the band of elevated surface temperature approximately coincided with the pressure plateau. Moreover, this region moved upstream and decreased in streamwise length as the angle of attack or Reynolds number was increased, following the changes expected in laminar separation bubbles [33]. It was concluded that due to decreased convective cooling in the reverse flow region, the higher temperature band could be used as an indicator for the presence of a laminar separation bubble.

Ricci \& Montelpare [28] expanded on this analysis, providing distributions of convective heat transfer intensity within the separated region on an internally heated RR3823HL airfoil. The temperature distribution was analyzed for Reynolds numbers of 60,000, 100,000, 150,000 and 200,000, between angles of attack of $-5^{\circ}$ and $12^{\circ}$. By considering radiation, conduction, and convection, a differential energy balance was performed on the thin aluminium coating applied to the airfoil surface. By controlling the amount of Joule heating provided, and measuring the resulting surface temperature, a distribution of the convective heat transfer coefficient on the airfoil surface was determined. The Stanton number along the chord was then calculated using edge velocity estimates from XFoil [93] as the characteristic velocity. The Stanton number distribution was used to estimate the locations of mean separation, transition, and reattachment. The separation location was stated to coincide with the inflection point in the Stanton number distribution, upstream of the absolute minimum. The location of transition was argued to lie at the location of absolute minimum Stanton number as Ricci \& Montelpare [28] suggested the maximum bubble height in this location resulted in minimum convective cooling. Lastly, mean reattachment 
was proposed to lie at the location of absolute maximum Stanton number, as the reattaching flow resulted in relatively high energy exchange between the outer flow and the model surface.

Dollinger et al. [23] used infrared thermography, surface pressure measurements, and oil film surface flow visualization to characterize separated regions on unheated airfoil and cylinder models. They used the temporal standard deviation of temperature fluctuations, or temperature fluctuation index (TFI), to characterize the separated region. The mean separation and reattachment locations were linked to regions of low and high TFI, respectively; however, no general threshold criteria were established. Dollinger et al. [94] were able to expand on this analysis and propose more concrete conclusions in a similar experiment. By comparing oil film flow visualization images with distributions of TFI, the mean separation location was shown to coincide with a sharp drop in TFI from the laminar region, a result of the decreased near-wall velocity within the separation bubble. Following separation, the TFI was shown to increase substantially at the location of reattachment. The increased fluctuations at reattachment were stated to be a result of turbulent characteristics of the boundary layer, and temporal oscillation of the reattachment location. The findings agreed with, and gave reasoning to, conclusions drawn in their previous study [23]. Moreover, using the temporal standard deviation of temperature fluctuations was shown to provide a viable estimate for both mean separation and reattachment on unheated surfaces with low thermal contrast.

\subsection{Heat Transfer Fundamentals}

As a fluid flow passes over an object, a thermal boundary layer will form due to differences in temperature between the fluid and the surface. Similar to the velocity boundary layer, the thermal boundary layer is diffusive in nature, with its development related to the fluid thermal diffusivity, $\kappa$. The amount of energy exchanged between the fluid and the surface depends on both the temperature difference and local flow behaviour [84]. The heat transfer potential due to local flow behaviour is quantified with the heat transfer coefficient, $h$, which depends on fluid properties, turbulence intensity, and flow speed. Originally formulated by Newton [95], the resulting convective heat transfer per unit area, $q_{\text {Conv }}^{\prime \prime}$, can be quantified using Newtons law of cooling:

$$
q_{\text {Conv }}^{\prime \prime}=h\left(T_{f}(x)-T_{w}(x)\right)
$$

Since the convective heat transfer coefficient, $h$, is dependent on flow characteristics, it varies with changes in boundary layer behaviour and local flow development [84]. The 
quantitative relationship between the convective heat transfer coefficient and flow field development can be obtained based on fundamental principles. By assuming a laminar flow with constant fluid properties, the Navier-Stokes equations and the energy equation become decoupled [96]. By further assuming negligible pressure gradient and negligible dissipation of work due to friction, the velocity and temperature fields become similar [96]. The similarity between the temperature and velocity fields allows for a proportional relationship between the convective heat transfer coefficient and the wall shear stress to be established, known as Reynolds Analogy [97,98]. First postulated in its simplest form by Reynolds [99], the Reynolds analogy states:

$$
\mathrm{St}=\frac{C_{f}}{2 \mathrm{Pr}}
$$

where:

$$
\begin{gathered}
\mathrm{St}=\frac{h}{\rho c_{p} U_{0}} \\
C_{f}=\frac{\tau_{w}}{\frac{1}{2} \rho U_{0}^{2}} \\
\operatorname{Pr}=\frac{\nu}{\kappa} .
\end{gathered}
$$

This relationship shows that for a laminar boundary layer flow with zero pressure gradient, the convective heat transfer coefficient is directly proportional to the local skin-friction [97, 98]. Equation 2.2 can also be modified for turbulent boundary layers, assuming a linear velocity profile in the near-wall region, as shown with the Prandtl-Taylor modification in Eq. 2.6:

$$
\mathrm{St}=\frac{C_{f}}{2 \operatorname{Pr}\left(1+\frac{U_{L}}{U_{0}}(\operatorname{Pr}-1)\right)}
$$

where $U_{L}$ is the upper limit of velocity in the linear near-wall velocity profile. The PrandtlTaylor modification collapses to Eq. 2.2 when $U_{L}=U_{0}$, which is the case for a laminar boundary layer.

For flow over a flat plate, the similarity between velocity and temperature fields also allows for empirical relationships describing the non-dimensional convective heat transfer coefficient as a function of other non-dimensional groups. For a laminar boundary layer, the non-dimensional convective heat transfer coefficient as a function of the streamwise coordinate is described by:

$$
\mathrm{Nu}_{\mathrm{x}}=0.332 \mathrm{Re}_{x}^{\frac{1}{2}} \operatorname{Pr}^{\frac{1}{3}}
$$

with a similar relationship for a turbulent boundary layer:

$$
\mathrm{Nu}_{\mathrm{x}}=0.0296 \operatorname{Re}_{x}^{\frac{4}{5}} \operatorname{Pr}^{\frac{1}{3}}
$$




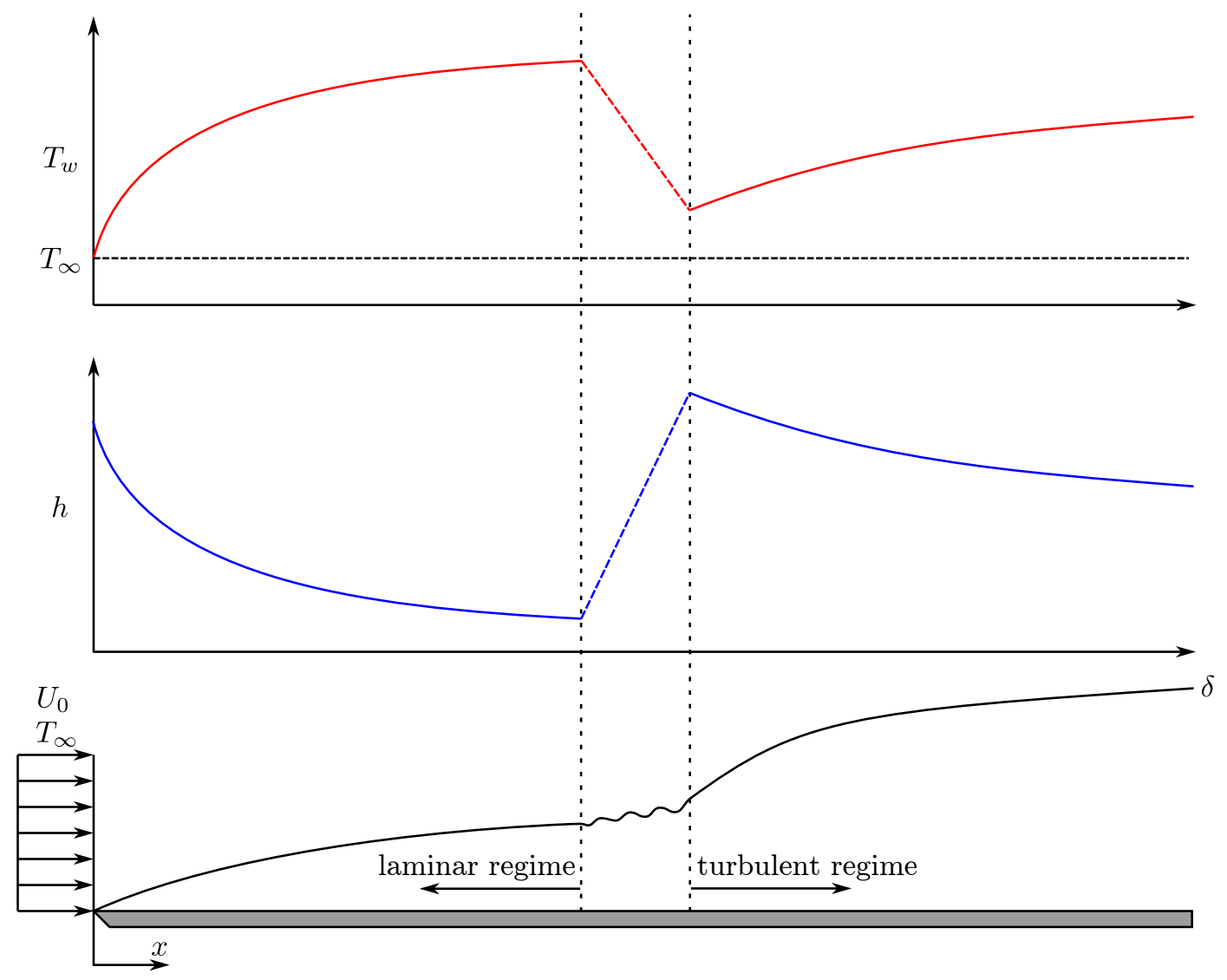

Figure 2.4: Development of a laminar boundary layer on a flat plate with zero pressure gradient undergoing natural transition to turbulent flow with corresponding distributions of convective heat transfer coefficient and surface temperature.

where:

$$
\mathrm{Nu}_{\mathrm{x}}=\frac{h x}{k}
$$

with $k$ being the thermal conductivity of the fluid [97, 98].

By rearranging Eqs. (2.7)-(2.9), the distribution of convective heat transfer coefficient can be estimated. This is shown schematically, along with the resulting wall temperature distribution, in Fig. 2.4, where the transition region is simplified as a linear interpolation between the laminar and turbulent regimes. As shown in Fig. 2.4, the convective heat transfer coefficient decreases non-linearly as the laminar boundary layer develops, reaching a minimum value around the onset of transition. After rapidly increasing and achieving a maximum value at the end the transition region, the convective heat transfer coefficient decreases as the turbulent boundary layer develops, at a rate slower than in the laminar region. Figure 2.4 shows the wall temperature being approximately equal to the free stream 
temperature at the leading edge, before increasing up to the onset of transition. The sharp increase in convective heat transfer coefficient within the transition region results in a rapid decrease of surface temperature. Upon reaching a minimum value near the end of transition, the surface temperature increases due to the decreasing convective heat transfer coefficient associated with development of the turbulent boundary layer. 


\section{Chapter 3}

\section{Experimental Methodology}

In this thesis, the characteristic heat transfer of laminar separation bubbles is examined experimentally with the use of infrared thermography. To analyze the heat transfer both qualitatively and quantitatively, two main experimental campaigns have been performed. First, a laminar separation bubble formed on a flat plate upstream of a vertically mounted fence was considered. Infrared thermography was used to obtain temperature fields, which were then compared to measurements of surface pressure, and two-component, planar particle image velocimetry. Secondly, a laminar separation bubble formed on a NACA 0018 airfoil was considered. The surface temperature measurements were then compared to measurements of velocity from two-component, planar particle image velocimetry. For both experimental campaigns, the Reynolds numbers were carefully selected such that valid measurements could be performed with each technique. The Reynolds numbers had to be high enough to provide measurable differences in surface temperature for the infrared camera, while being low enough such that sufficient spatial resolution could be achieved with each measurement technique. The flow conditions, and the corresponding measurements performed at each condition, are summarized in Tables 3.1 and 3.2 for the flat plate and airfoil experiments, respectively. In this chapter, the experimental facility, models, and techniques are described in detail.

Table 3.1: Summary of the flow conditions and corresponding measurements performed on the flat plate geometry.

\begin{tabular}{cccccccccc}
\hline \hline $\operatorname{Re}_{L}$ & 24,000 & 27,000 & 30,000 & 33,000 & 36,000 & 39,000 & 42,000 & 45,000 & 48,000 \\
\hline Pressure & $\bullet$ & $\bullet$ & $\bullet$ & $\bullet$ & $\bullet$ & $\bullet$ & $\bullet$ & $\bullet$ & $\bullet$ \\
PIV & $\bullet$ & $\bullet$ & & & $\bullet$ & & & $\bullet$ & \\
IRT & $\bullet$ & $\bullet$ & $\bullet$ & $\bullet$ & $\bullet$ & $\bullet$ & $\bullet$ & $\bullet$ & $\bullet$ \\
\hline \hline
\end{tabular}

- Indicates that measurement was performed 
Table 3.2: Summary of the flow conditions and corresponding measurements performed on the airfoil geometry.

\begin{tabular}{lc|ccc}
\hline \hline & PIV & \multicolumn{3}{c}{ IRT } \\
& $\alpha=4^{\circ}$ & $\alpha=2^{\circ}$ & $\alpha=4^{\circ} \quad \alpha=6^{\circ}$ & $\alpha=8^{\circ}$ \\
\hline $\operatorname{Re}_{c}=70,000$ & & & $\bullet$ & \\
$\operatorname{Re}_{c}=80,000$ & $\bullet$ & $\bullet$ & $\bullet$ & $\bullet$ \\
$\operatorname{Re}_{c}=90,000$ & & & $\bullet$ & $\bullet$ \\
$\operatorname{Re}_{c}=100,000$ & & $\bullet$ & \\
$\operatorname{Re}_{c}=110,000$ & & & $\bullet$ & \\
$\operatorname{Re}_{c}=120,000$ & $\bullet$ & $\bullet$ & $\bullet$ & $\bullet$ \\
$\operatorname{Re}_{c}=130,000$ & & & $\bullet$ & \\
$\operatorname{Re}_{c}=140,000$ & & & $\bullet$ \\
$\operatorname{Re}_{c}=150,000$ & & $\bullet$ & \\
\hline \hline
\end{tabular}

- Indicates that measurement was performed

\subsection{Experimental Setup}

\subsubsection{Wind Tunnel Facility}

All experiments were performed in the closed-loop wind tunnel at the University of Waterloo, which is shown schematically in Fig. 3.1. The flow is generated with a six-blade fan attached to an axial motor, controlled via variable frequency drive. Flow conditioning is achieved as the flow passes through the settling chamber, consisting of an aluminium honeycomb structure followed by five turbulence reducing screens at the upstream side of the 9:1 contraction. The test section is $2.44 \mathrm{~m}$ in length, with a cross-section measuring $0.61 \mathrm{~m} \times 0.61 \mathrm{~m}$. The test section walls are made of tempered glass to allow for easy optical access. The velocity in the test section is set using the static pressure drop across the contraction, which was calibrated against a Pitot-static tube placed in an empty test section. The resulting uncertainty in the free stream velocity was calculated to be less than $2 \%$ for each flow condition (Appendix D.1). Kurelek [53] showed that the flow uniformity within the test section varied less than $0.4 \%$ at a free stream velocity of $9.6 \mathrm{~m} / \mathrm{s}$. The turbulence intensity was measured by Istvan \& Yarusevych [11] to be approximately $0.06 \%$.

\subsubsection{Flat Plate Model}

The flat plate model used in these experiment is shown in Fig. 3.2. The plate is constructed in three pieces, with a modular fence that allows for variable placement. The mid-span point at the leading edge corresponds to the origin of the coordinate system used for data 


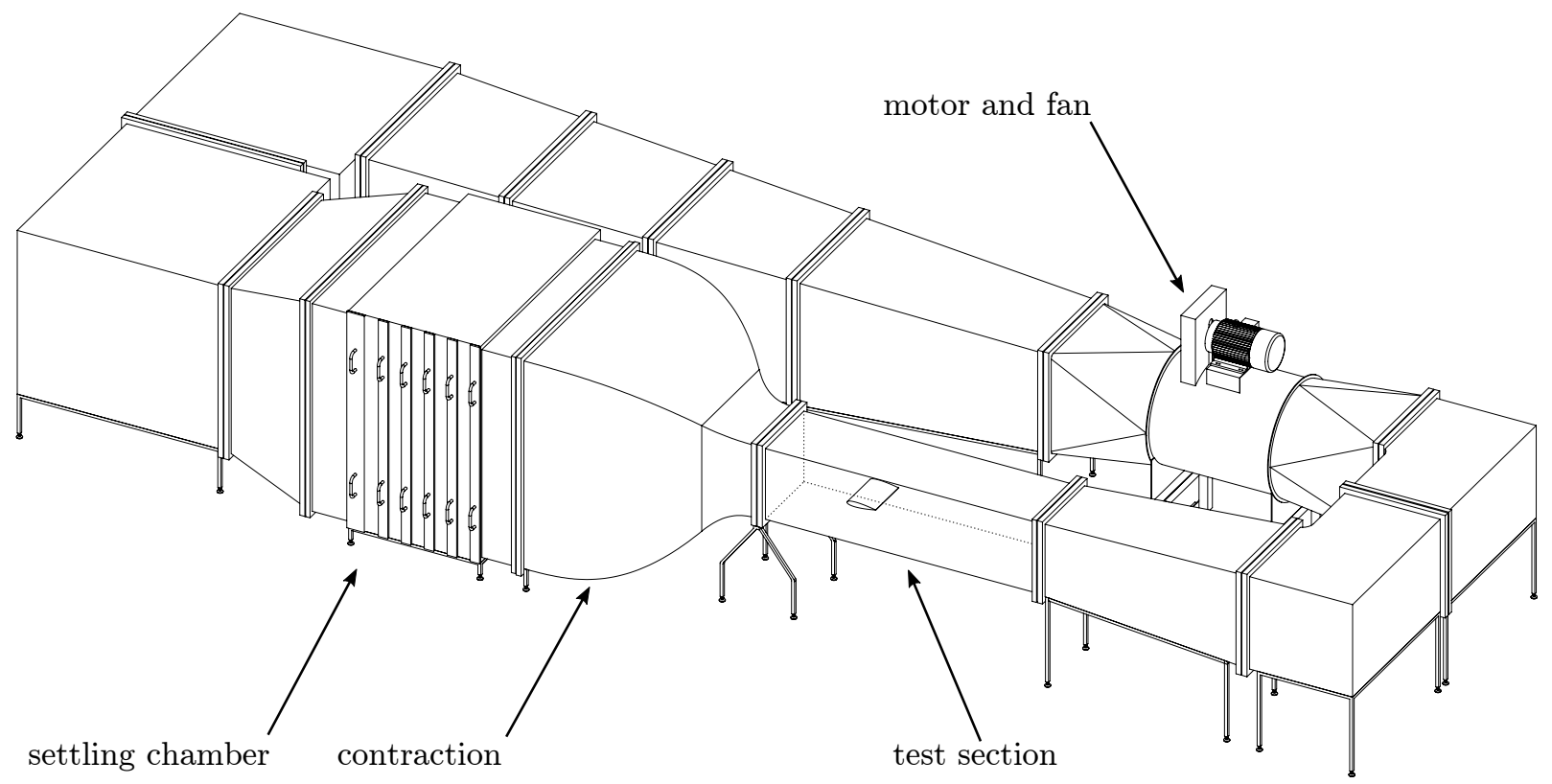

Figure 3.1: Recirculating wind tunnel at the University of Waterloo.

presentation, with $x, y$, and $z$ coordinates pointing in the streamwise, wall-normal, and spanwise directions, respectively. The aluminium nose portion is $0.500 \mathrm{~m}$ long and has a super-elliptical shaped leading edge to reduce the receptivity of the boundary layer to sound fluctuations in the free-stream [100]. The middle section of the flat plate is $0.525 \mathrm{~m}$ long and is made of Lexan due to its low thermal conductivity. To increase emissivity of the top surface, it was painted with three coats of flat black paint. The trailing edge of the flat plate is also made of aluminium and is $0.500 \mathrm{~m}$ long, resulting in an overall length of $1.525 \mathrm{~m}$. The flat plate model is $0.600 \mathrm{~m}$ wide, with $0.005 \mathrm{~m}$ of foam padding added at the side edges such that the model spans the entire test section width. Three NACA 0012 shaped legs were used to support the flat plate model in the test section. The supports are $0.190 \mathrm{~m}$ tall, with two placed beneath the nose section and one placed in the center of the tail section.

The fence used in these experiments was constructed out of Lexan, such that it did not affect the laser sheet used in PIV. The fence had a height, $L$, of $0.053 \mathrm{~m}$ and a thickness of $0.010 \mathrm{~m}$. The modular design allowed for the fence to be placed at the downstream edge of the Lexan centerpiece, with the upstream face of the fence located $1.025 \mathrm{~m}(19.34 L)$ downstream from the leading edge. The trailing edge flap was set at an angle of $0^{\circ}$, since the use of a vertical fence ensured a desirable stagnation point placement.

Measurements of static surface pressure were performed through a total of 54 embedded pressure taps with a diameter of $0.4 \mathrm{~mm}$. The first six pressure taps are in the nose section 
(a)

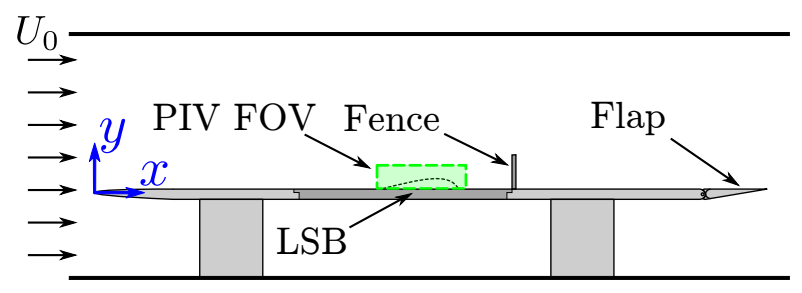

(b)

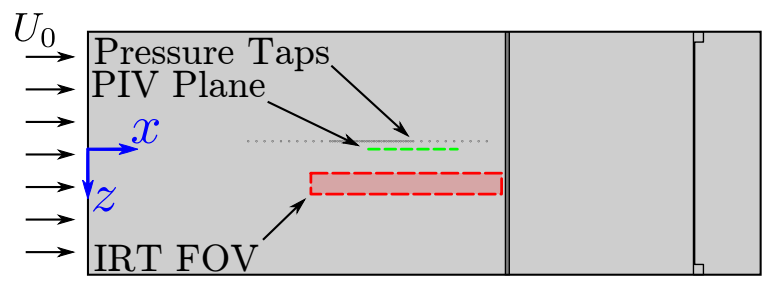

Figure 3.2: (a) Side, and (b) top view of the flat plate model. The sections corresponding to different plate materials are not shown in (b) for clarity.

of the model, followed by 48 in the middle section. The first pressure tap is at a location of $0.394 \mathrm{~m}(7.43 \mathrm{~L})$ downstream of the leading edge, with $20.1 \mathrm{~mm}$ spacing between the first ten taps. Pressure taps 11-45 are more densely packed with streamwise spacing of $6.0 \mathrm{~mm}$, followed by taps $46-54$ with spacing of $20.1 \mathrm{~mm}$. The pressure taps and their relative spacing is highlighted in Fig. 3.2b.

\subsubsection{Airfoil Model}

The airfoil model used in these experiments is shown in Fig. 3.3. A NACA 0018 profile was used with chord and spanwise lengths of $0.20 \mathrm{~m}$ and $0.60 \mathrm{~m}$, respectively. The mid-span location at the leading edge was defined as the origin, and a surface attached coordinate system was employed for data presentation. The $x$-coordinate runs from the origin, along the surface, in the streamwise direction. The $y$-coordinate points normal to the suction surface, and the $z$-coordinate points in the spanwise direction. The airfoil model was 3D printed with black polycarbonate-ABS plastic. The design consisted of a $5 \mathrm{~mm}$ solid polycarbonate-ABS shell, with a primarily hollow core (Fig. 3.3c). The core was made up of $70 \%$ air with $30 \%$ polycarbonate-ABS by volume to maintain structural integrity. The thin shell, air-filled, design ensured that conduction into the model was minimized. After the model was 3D printed, it was sanded in multiple stages to rid all surface imperfections introduced in the printing process. The final sandpaper used was 600-grit, resulting in a smooth, matte surface finish.

The zero incidence was establish based on the geometric zero angle of attack of the employed symmetric airfoil model to within $0.1^{\circ}$. Relative to the zero angle of attack, the angle was set using a digital protractor, with a resolution of $0.1^{\circ}$, which rotated with the airfoil. The axis of rotation was $0.25 c$ downstream of the leading edge, located $3 c$ downstream of the contraction outlet.

The highlighted measurements presented in this thesis are at an angle of attack of $4^{\circ}$ and Reynolds numbers of 80,000 and 120,000, with a full list of flow conditions shown in 
(a)

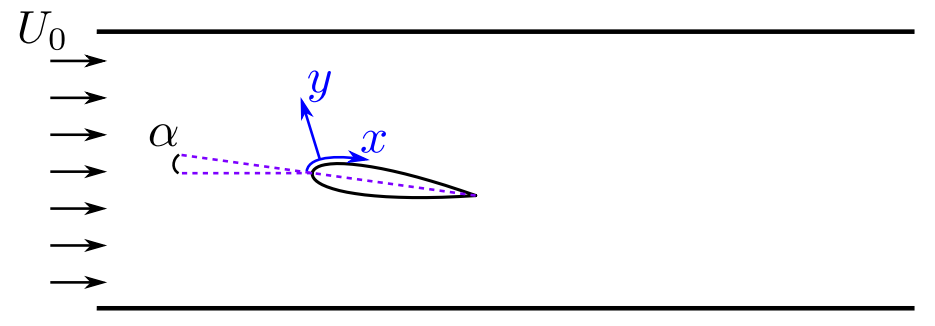

(b)

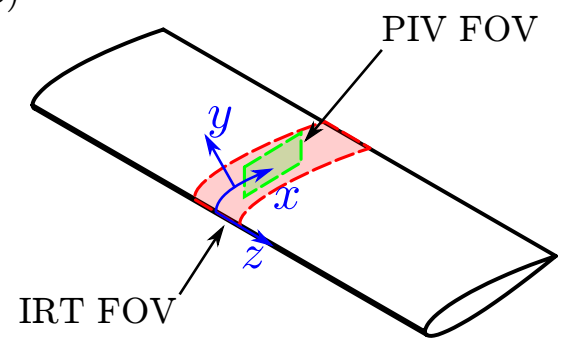

(c)

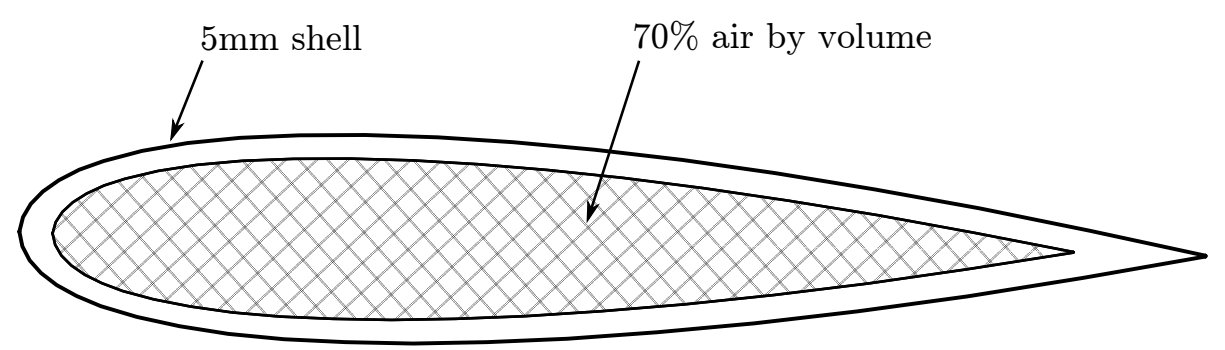

Figure 3.3: (a) Side, (b) isometric, and (c) section view of the airfoil model.

Table 3.2. An angle of attack of $4^{\circ}$ was selected as a laminar separation bubble commonly forms in the mid-to-aft portion of the airfoil, and has been employed in several other studies $[53,101,102]$. The Reynolds numbers of 80,000 and 120,000 were selected as they are at the lower and upper end of conditions resulting in a laminar separation bubble which were measurable with the selected measurement equipment. At an angle of attack of $4^{\circ}$, the blockage ratio without end caps installed was found to be approximately $6.0 \%$. It has been shown by Boutilier \& Yarusevych [103] that a blockage ratio on this order has a negligible effect on the development of the laminar separation bubble. End plates were also not implemented as Boutilier \& Yarusevych [103] showed that installing end plates had no effect on the flow uniformity over more than $40 \%$ of the central span for comparable conditions.

\subsection{Measurement Techniques}

Three different measurement techniques were employed during this study. Static pressure measurements were used in the flat plate study, and are described in Section 3.2.1. Particle image velocimetry and infrared thermography were used in both experimental investigations and are described in Section 3.2.2 and Section 3.2.3, respectively. 


\subsubsection{Pressure Measurements}

The distribution of static pressure on the flat plate model surface was measured in the first experimental investigation. The static pressures were measured with two Setra Model 239 high-accuracy differential pressure transducers, with a full range of $\pm 250 \mathrm{~Pa}$. Using flexible PVC tubing, the pressure taps were connected to a Scanivalve mechanical multiplexer, which measured the static pressure at each location relative to the static pressure at the first pressure tap. The Scanivalve mechanical multiplexer used a multiplexing configuration developed by Boutilier [101], allowing for the pressures to be measured consecutively, two taps at a time. The Setra pressure transducers were connected to the Scanivalve mechanical multiplexer and were sampled with an NI USB-6259 DAQ. The output voltage of the transducers was measured at a frequency of $5,000 \mathrm{~Hz}$ with 20,000 samples for each tap. Before the wind tunnel was turned on, the transducers were zeroed at the quiescent condition. Once the wind tunnel was turned on, the pressure in the PVC tubing was allowed $60 \mathrm{~s}$ to equalize before the first pressures were measured. Following this, a wait time of $25 \mathrm{~s}$ was employed between subsequent taps to allow for pressure equalization to occur within the multiplexer device. The associated uncertainty in the static pressure measurements was found to be less than $3 \%$ of the dynamic pressure for all flow conditions (Appendix D.2).

\subsubsection{PIV Measurements}

Two-component, planar PIV was employed on both geometries in order to reveal the location and extent of the laminar separation bubble. For all experiments, the flow was seeded with a water-glycol based fog produced with a Rosco vapour fog machine, resulting in particles with a mean diameter on the order of $1 \mu \mathrm{m}$. The light beam emitted from a Photonics DM20-527 high-speed Nd:YLF pulsed laser was passed through an optical setup to illuminate the fog particles in a sheet along the plane of interest. Photron SA4 high-speed cameras were then used to record images of the flow. A LaVision timing unit controlled through LaVision's DaVis 8 software was used to synchronize the laser pulse with the image acquisition.

For experiments on the flat plate, as shown in Fig. 3.5a, a single Photron SA4 highspeed camera was used to capture the development of the laminar separation bubble formed upstream of the vertically mounted fence at the mid-span plane $(z=0)$. The laser beam was oriented normal to the test section downstream of the flat plate, as shown in Fig. 3.5a. The laser beam was pointed at the optics setup for conditioning, which was mounted on a breadboard. The beam was passed through a $90^{\circ}$ turn mirror followed by a $-75 \mathrm{~mm}$ cylindrical lens, placing a laser sheet, approximately $1 \mathrm{~mm}$ thick, in the field of interest. A Nikon $200 \mathrm{~mm}$ focal length macro lens was used with an $f_{\#}$ of 5.6. The Photron camera 
was placed on a 3-axis traverse system to allow for controlled and repeatable positioning. The image sensor was cropped to $1024 \times 512 \mathrm{px}$ and the camera was moved closer to the flow as the Reynolds number was increased, such that the spatial resolution within the separation bubble was maximized. A total of five fields of view were captured in the streamwise direction (Fig. 3.4a), with an overlap of $3 \mathrm{~mm},(3.4 \%, 4.0 \%$, and 5.5\% for Reynolds numbers 27,000,36,000, and 45,000, respectively) allowing for the time-averaged velocity fields to be stitched together. A total of 2,500 images were acquired in double frame mode at a frequency of $1,000 \mathrm{~Hz}$. The $\Delta t$ was selected such that the temporal average particle displacement in the free-stream was approximately 15px. A summary of the important PIV parameters for the flat plate experiments is shown in Table 3.3.

For the experiments performed on the airfoil geometry, as shown in Fig. 3.5b, two Photron SA4 high-speed camera were used to capture the laminar separation bubble forming on the suction side at the mid-span plane $(z=0)$. Again, the laser beam was oriented normal to the test section, downstream of the airfoil, as shown in Fig. 3.5b. The laser beam was pointed at the optics setup for conditioning, which was mounted on a breadboard. The beam was passed through a $90^{\circ}$ turn mirror followed by a $-75 \mathrm{~mm}$ cylindrical lens, placing a laser sheet, approximately $1 \mathrm{~mm}$ thick, in the field of interest. Nikon $200 \mathrm{~mm}$ focal length macro lenses were used with an $f_{\#}$ of 4 . The Photron cameras were placed on a fixed optical rail setup on both sides of the test section and were adjusted at both Reynolds numbers such that the spatial resolution within the bubble was maximized. The camera sensors were cropped to $1024 \times 512$ px and an overlap of $13 \%$ and $20 \%$ was maintained for Reynolds numbers of 80,000, and 120,000, respectively (Fig. 3.4b). A total of 5,000 images were acquired in double frame mode at a frequencies of $1,500 \mathrm{~Hz}$ and $100 \mathrm{~Hz}$ for each Reynolds number. The dataset at $100 \mathrm{~Hz}$ allowed for time-averaged analysis as the $50 \mathrm{~s}$ measurement cycle captured low-frequency oscillations associated with the laminar separation bubble, while the $1,500 \mathrm{~Hz}$ dataset allowed for time-resolved analysis of the flow dynamics. The $\Delta t$ for each Reynolds number was selected such that the temporal average particle displacement in the free-stream was approximately 16px. A summary of the important PIV parameters for the airfoil experiments is shown in Table 3.4.

For all PIV measurements, the acquisition and processing was performed in LaVision's DaVis 8 software. The particle images were pre-processed using temporal sliding minimum subtraction to minimize surface reflections. The resulting images were processed with a multi-pass cross-correlation algorithm with window deformation [104]. The final window size was $16 \times 16$ px with an overlap of $75 \%$. In order to provide better spatial resolution at the point of separation, a multi-pass sum of correlations (SOC) algorithm [105] with a window size of $4 \times 4$ px and an overlap of $75 \%$ was implemented. The different fields of view and their corresponding processing algorithms for each experiment are shown in Fig. 3.4. 
(a)

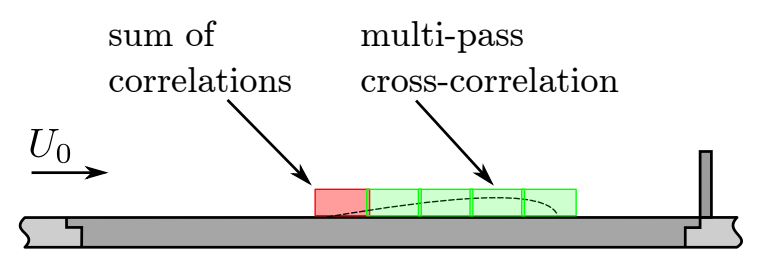

(b)

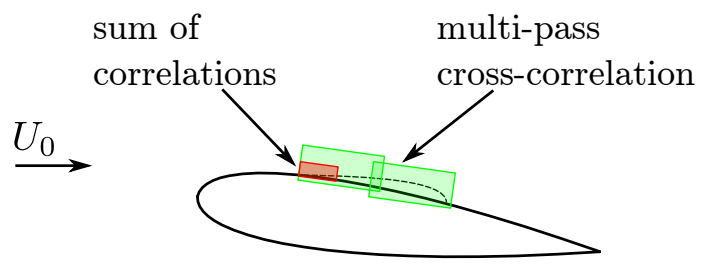

Figure 3.4: PIV fields of view and corresponding correlation algorithms used for (a) flat plate, and (b) airfoil experiments.

Table 3.3: Important PIV parameters for flat plate experiments.

\begin{tabular}{ccccc}
\hline \hline Parameter & $\operatorname{Re}_{L}=27,000$ & $\mathrm{Re}_{L}=36,000$ & $\mathrm{Re}_{L}=45,000$ & Unit \\
\hline Sampling Rate & & 1000 & & $\mathrm{~Hz}$ \\
Images Acquired & 100 & 2500 & 40 & $\mathrm{us}$ \\
Frame Separation & & 60 & & $\mathrm{~mm}$ \\
$f_{\#}$ & 5.6 & & $\mathrm{px}$ \\
Lens Focal Length & & 200 & 0.71 & \\
Sensor Resolution & 0.44 & $1024 \times 512$ & $133 \times 14$ & $\mathrm{~mm}$ \\
Magnification Factor & $218 \times 23$ & 0.53 & $2.51 \times 0.26$ & $L$ \\
Combined Field of View & $4.11 \times 0.43$ & $3.45 \times 0.36$ & & $\mathrm{px}$ \\
Final Window Size & & $16 \times 16$ & $\mathrm{px}$ \\
SOC Final Window Size & & $4 \times 4$ & 0.113 & $\mathrm{~mm}$ \\
Vector Pitch & 0.180 & 0.152 & 15 & $\mathrm{px}$ \\
Outer Flow Displacement & 16 & 15 & & \\
\hline \hline
\end{tabular}

The final vector fields were stitched together with a cosine weighted blending factor in the overlap region. The vector fields for the airfoil geometry were then interpolated onto the surface attached coordinate system (Fig. 3.3).

\subsubsection{Infrared Measurements}

The distribution of surface temperature for each model was measured with an Optris PI640 infrared camera, with a thermal sensitivity of $75 \mathrm{mK}$. The image sensor was cropped to a size of $640 \times 120 \mathrm{px}$, from the full size of $640 \times 480 \mathrm{px}$, allowing for an increased frame rate. A $60^{\circ} \times 33^{\circ}$ lens was used, enabling the region of interest to be captured within the infrared field of view (Figs. 3.2b and 3.3b). For experiments on the flat plate, the field of view spanned from 545 to $1016 \mathrm{~mm}(10.28 \mathrm{~L}-19.17 \mathrm{~L})$ from the leading edge, with a width of $84 \mathrm{~mm}(1.58 \mathrm{~L})$ (Fig. 3.2b). The center of the field of view was offset by $83.5 \mathrm{~mm}$ 
Table 3.4: Important PIV parameters for airfoil experiments.

\begin{tabular}{|c|c|c|c|}
\hline Parameter & $\operatorname{Re}_{c}=80,000$ & $\operatorname{Re}_{c}=120,000$ & Unit \\
\hline Sampling Rate & \multicolumn{2}{|c|}{$100 \& 1500$} & $\mathrm{~Hz}$ \\
\hline Images Acquired & \multicolumn{2}{|c|}{5000} & \\
\hline Frame Separation & 90 & 50 & us \\
\hline$f_{\#}$ & \multicolumn{2}{|c|}{4} & \\
\hline Lens Focal Length & \multicolumn{2}{|c|}{200} & $\mathrm{~mm}$ \\
\hline Sensor Resolution & \multicolumn{2}{|c|}{$1024 \times 512$} & $\mathrm{px}$ \\
\hline Magnification Factor & 0.49 & 0.58 & \\
\hline \multirow{2}{*}{ Combined Field of View } & $70 \times 14$ & $64 \times 12$ & $\mathrm{~mm}$ \\
\hline & $0.35 \times 0.07$ & $0.32 \times 0.06$ & $c$ \\
\hline Final Window Size & \multicolumn{2}{|c|}{$16 \times 16$} & $\mathrm{px}$ \\
\hline SOC Final Window Size & \multicolumn{2}{|c|}{$4 \times 4$} & $\mathrm{px}$ \\
\hline Vector Pitch & 0.164 & 0.137 & $\mathrm{~mm}$ \\
\hline Outer Flow Displacement & 16 & 17 & $\mathrm{px}$ \\
\hline
\end{tabular}

$(1.58 L)$ from the plate center as the flat plate has a channel milled for the pressure tap installations at the center-span. For the airfoil experiments, the infrared camera captured a region along the entire chord, with a width of $35 \mathrm{~mm}(0.175 c)$ centered at the mid-span location (Fig. 3.3b).

Since the test section walls do not transmit radiation within the spectral range of the infrared camera $(7.5 \mu \mathrm{m}-13 \mu \mathrm{m})$, a $45 \mathrm{~mm}$ view hole was drilled in the top surface of the test section, which allowed for placement of the infrared camera directly above the field of interest. The camera was placed on a customized mount, resulting in the lens of the camera being flush with the inner wall of the test section such that it did not disturb the flow. To increase thermal contrast, the surface was externally heated using a set of $3 \times 500 \mathrm{~W}$ halogen lamps placed $0.40 \mathrm{~m}$ above the test section. Figure 3.5 shows the placement of the infrared camera and the halogen lamps with respect to the test section. Once the halogen lamps were turned on, the model was given sufficient time to heat up and achieve a steady-state temperature before the image acquisition process was initiated. The transient temperature distribution for the heating of each model is displayed in Appendix A. Measurements of the surface temperature were acquired in both quiescent and flow conditions. Measurements in quiescent conditions allowed for evaluation of non-uniform heating and conduction losses, whereas in-flow measurements allowed for analysis of the convective cooling within the region of interest.

For the airfoil experiments, the infrared images were transformed to the surface attached coordinate system at each angle of attack. Markers were placed at $0.1 \mathrm{c}$ increments along the chord and imaged with the infrared camera. The location of the markers in the 
(a)

(b)
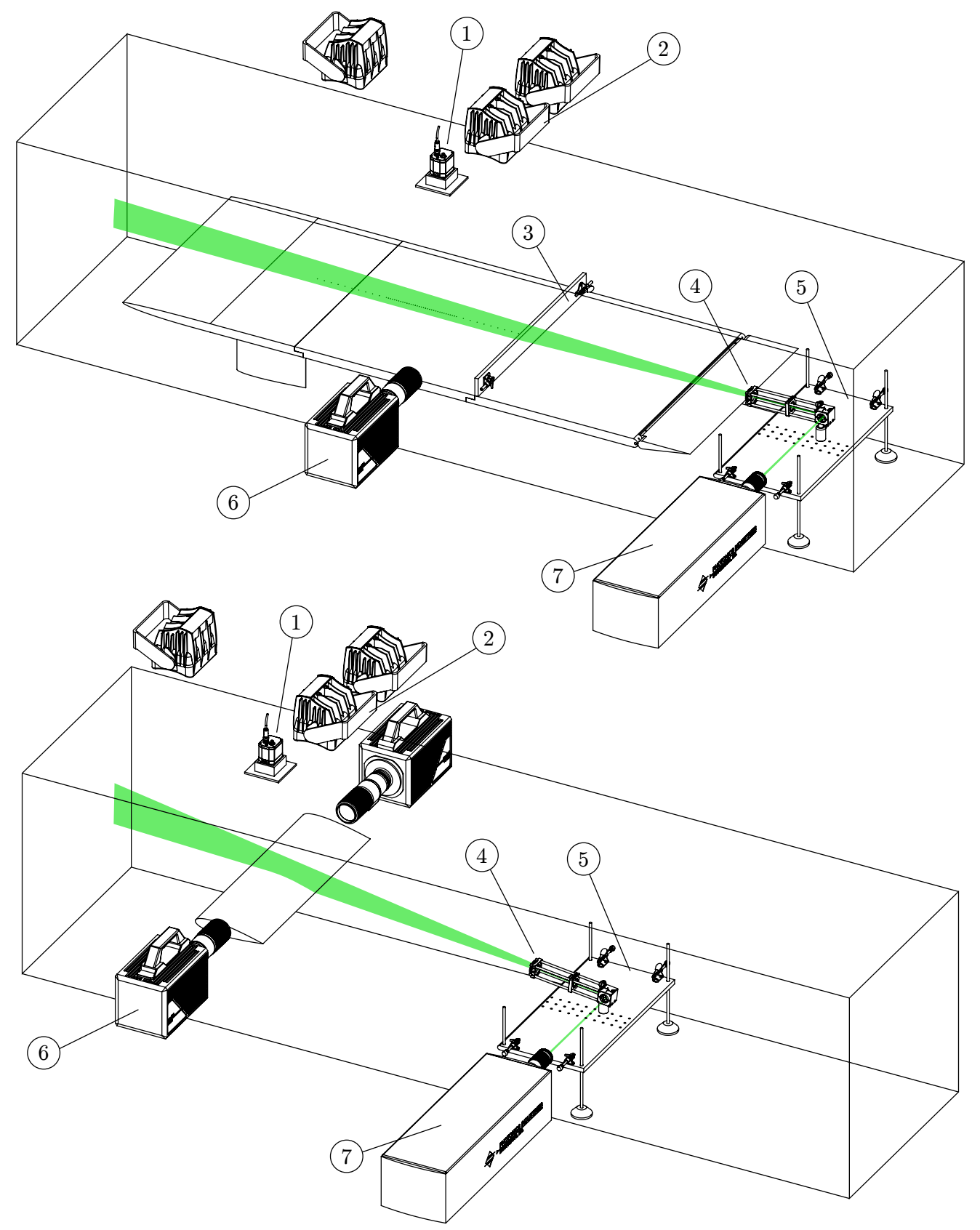

Figure 3.5: Configuration of experimental equipment for the (a) flat plate, and (b) airfoil experiments. (1) Infrared camera and customized mount; 2 Halogen lamps; 3 Vertically mounted fence; (4) Optics; 5 Breadboard; 6 High-speed camera; 7 Laser. 
Table 3.5: Important infrared thermography parameters for each experimental investigation.

\begin{tabular}{|c|c|c|c|}
\hline Parameter & Flat Plate & Airfoil & $\overline{\text { Unit }}$ \\
\hline Camera & \multicolumn{2}{|c|}{ Optris PI640 } & \\
\hline Sensor Resolution & \multicolumn{2}{|c|}{$640 \times 120$} & $\mathrm{px}$ \\
\hline Thermal Sensitivity & \multicolumn{2}{|c|}{75} & $\mathrm{mK}$ \\
\hline Spectral Range & \multirow{2}{*}{\multicolumn{2}{|c|}{$\begin{array}{l}7.5-13 \\
60^{\circ} \times 33^{\circ}\end{array}$}} & $\mu \mathrm{m}$ \\
\hline Lens & & & \\
\hline Sampling Rate & \multicolumn{2}{|c|}{10} & $\mathrm{~Hz}$ \\
\hline Images Acquired & \multicolumn{2}{|c|}{1000} & \\
\hline Physical Field of View & \multirow{2}{*}{\multicolumn{2}{|c|}{$\begin{array}{c}8.89 L \times 1.58 L \quad 1.00 c \times 0.175 c \\
1500\end{array}$}} & $\mathrm{~mm}$ \\
\hline Heating Applied & & & $\mathrm{W}$ \\
\hline
\end{tabular}

${ }^{*}$ Streamwise direction is cropped to size of the airfoil

thermal images were then related to the corresponding $x / c$ locations and a fourth-order polynomial fit was used for interpolation. For all experiments, the image acquisition was triggered using the Optris Connect software with post processing performed in MATLAB. The temperatures were adjusted based on an ambient calibration between the infrared camera and a thermocouple, correcting for differences in perceived emissivity on the model surface. For each condition, a total of 1,000 images were collected at a frequency of $10 \mathrm{~Hz}$. The temperature fields were temporally averaged to remove thermal noise as suggested by Ricci \& Montelpare [28]. A spatial filter with a kernel of 5px was then applied to the resulting temperature fields to mitigate spurious artifacts. An additional 5px spatial filter was applied to all computed line distributions to allow for clearer data presentation. A summary of all parameters for the infrared measurements is given in Table 3.5.

\subsection{Expected Thermal Behaviour of a Laminar Sepa- ration Bubble}

In order to estimate the locations of separation, transition, and reattachment for the laminar separation bubble using infrared measurements, a methodology for doing so must first be established. The following section will present the expected behaviour of convective heat transfer intensity and surface temperature beneath the laminar separation bubble. The laminar separation bubble will be broken into several distinct regions, as shown in Fig. 3.6, which correspond to different flow developments. The convective heat transfer within the different regions will be characterized based on comparisons to similar, well-studied flows. 


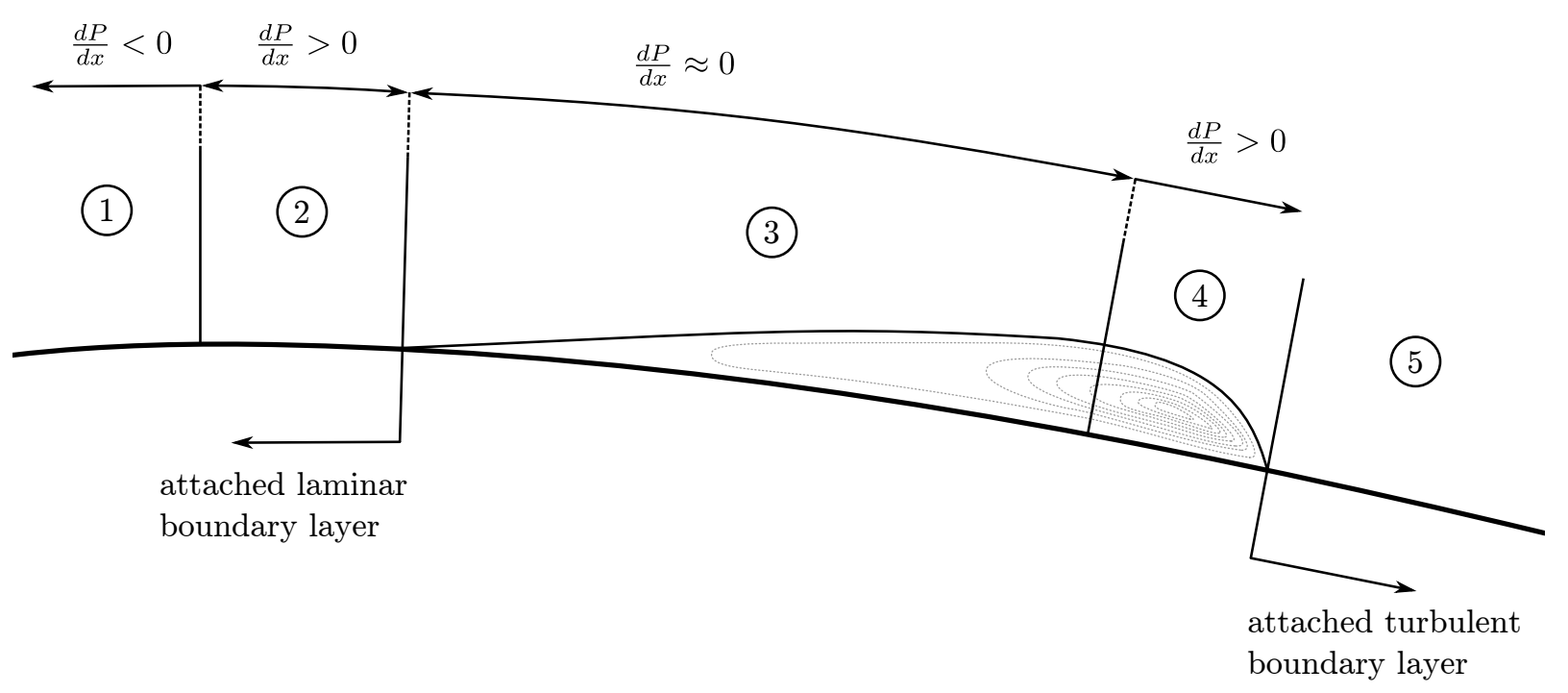

Figure 3.6: Pressure gradient and boundary layer characteristics in a laminar separation bubble. (1) Laminar flow subject to a favourable pressure gradient; (2) laminar flow subject to an adverse pressure gradient; (3) dead-air region; (4) later stages of transition leading to reattachment; (5) developing turbulent boundary layer.

It should be noted that all descriptions provided will be based the assumption of a constant wall heat flux, with a temperature profile of $T_{w}(x)$. The film temperature, $T_{f}$ is assumed to be constant and equal to the free-stream fluid temperature $T_{\infty}$, with $T_{w}>T_{\infty}$.

Upstream of the separation point, in regions (1) and (2) in Fig. 3.6, the boundary layer can be reasonably modelled as an attached laminar boundary layer on a flat plate. The heat transfer within a laminar flat plate boundary layer is well-studied and has been shown to follow theoretical predictions [106]. As shown in Eq. 2.7, the analytical solution of this flow predicts the convective heat transfer coefficient decreasing at a rate inversely proportional to the square root of the local Reynolds number $[97,98]$. The decreasing convective heat transfer coefficient results in a simultaneous increase in the surface temperature due to Eq. 2.1 paired with the assumption of a constant wall heat flux. The growth of a laminar boundary layer with an increasing surface temperature is shown in Fig. 3.7. Figure 3.7 depicts the situation for air with $\operatorname{Pr} \approx 0.7$, where the thermal boundary layer thickness exceeds the velocity boundary layer thickness at a given location.

The difference between regions (1) and (2) in Fig. 3.6 is due to the presence of a favourable and adverse pressure gradient, respectively. In region (1), the laminar boundary layer is subject to a favourable pressure gradient, which decreases the boundary layer thickness and increases the wall-shear stress and convective heat transfer coefficient [107110]. As the flow approaches the suction peak, the favourable pressure gradient diminishes, 
(a)
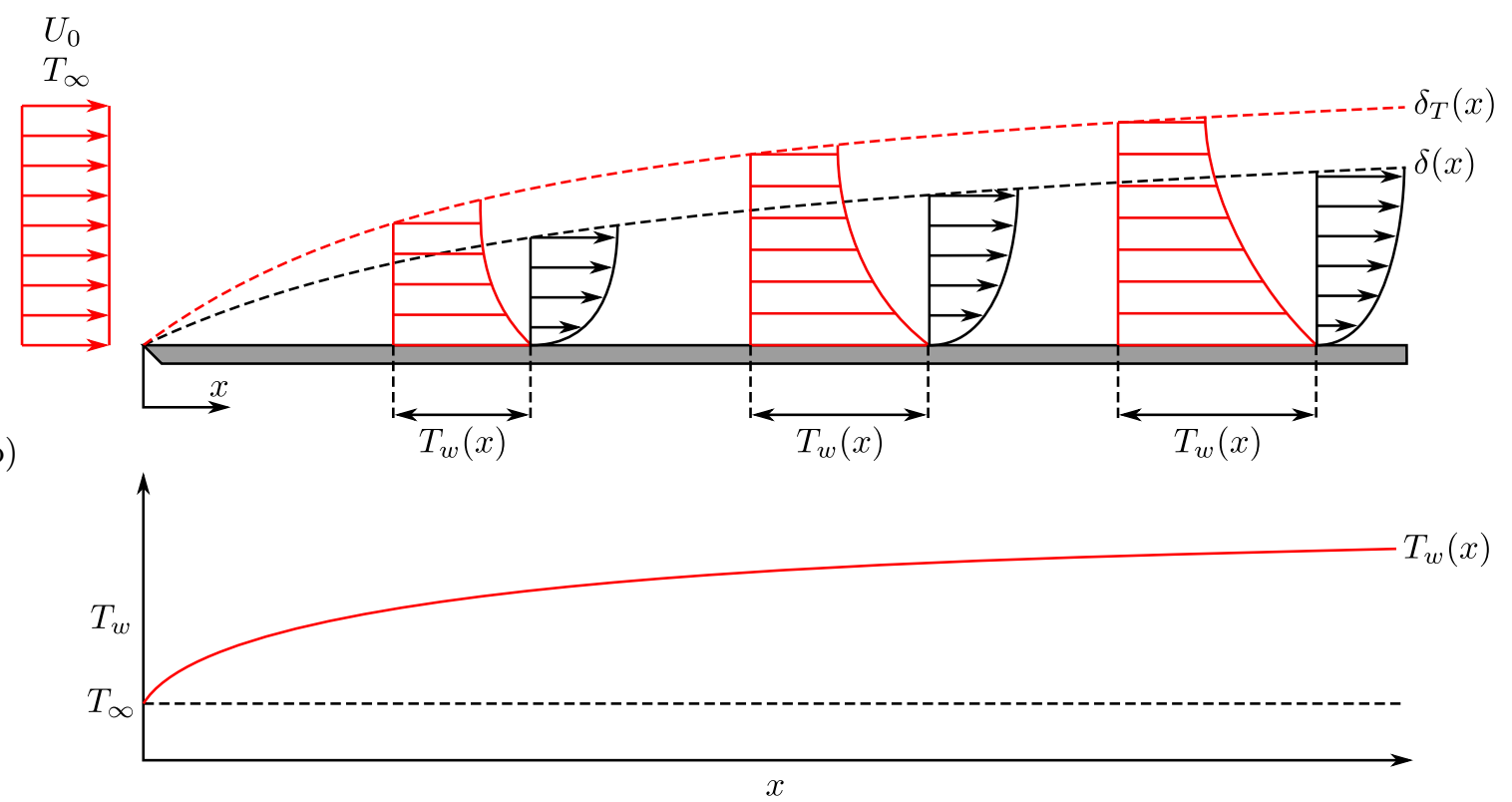

Figure 3.7: (a) Formation of thermal and velocity boundary layers on a flat plate subject to a constant heat flux, and (b) corresponding wall temperature development along the streamwise direction.

reaching a zero pressure gradient at the suction peak, and an adverse pressure in region (2). The adverse pressure gradient in region (2) decelerates the near-wall flow, increasing the rate of boundary layer growth and decreasing the local skin-friction $[107,111,112]$. The skin-friction decreases along the chord, reaching a value of zero at the point of separation [46,49, 113-115]. The assumptions established in Section 2.4 while deriving the Reynolds analogy fail to hold true for flows subject to a pressure gradient. Therefore, the location corresponding to zero skin-friction does not result in a minimum of the convective heat transfer coefficient. Here, the convective heat transfer coefficient decreases, and its streamwise gradient reaches a minimum at the point of separation [114].

Region 3 in Fig. 3.6 coincides with the dead-air region where the streamwise pressure gradient is approximately zero $[6,33,49,50]$. In this region, the shear stress is negligibly small due to the slow-moving or stagnant fluid in the near-wall region [50,114, 115]. With the onset of transition, amplified velocity fluctuations in the separated shear layer begin to enhance momentum and energy exchange near the surface, causing a rapid increase in the shear stress at the wall. This results in a simultaneous rise in the convective heat transfer coefficient, which reaches a maximum streamwise gradient around the mean transition location [114]. Similar to the transition region described for a flat plate in Section 2.4, the 
rapidly increasing convective heat transfer coefficient in the transition region results in a rapid decrease in the surface temperature due to Newtons law of cooling Eq. 2.1.

The location of mean reattachment lies between regions (4) and 5 in Fig. 3.6. As the flow reattaches to the surface, fluid entrained from the outer flow periodically impinges on the surface causing a further increase in the convective heat transfer coefficient downstream of the mean transition location, reaching a peak value in the vicinity of mean reattachment. Downstream of reattachment, the attached turbulent boundary layer develops and the skin-friction quickly recovers before undergoing an exponential decay with growth of the turbulent boundary layer $[46,50,114,115]$. The developing turbulent boundary layer in region 5 is similar to a turbulent boundary layer on a flat plate, with empirical correlations available for the convective heat transfer coefficient as described in Eq. 2.8 [97,98]. The expected variation of convective heat transfer coefficient and surface temperature within this region are shown in Fig. 2.4. 


\section{Chapter 4}

\section{Streamwise Flow Development of a Laminar Separation Bubble on a Flat Plate}

In this chapter, laminar separation bubbles formed on a flat plate, upstream of a vertically mounted fence, are investigated. Measurements are performed at Reynolds numbers $\left(\operatorname{Re}_{L}\right)$, based on the fence height, between 24,000 and 48,000, with the $\operatorname{Re}_{L}=27,000$, $\operatorname{Re}_{L}=36,000$, and $\operatorname{Re}_{L}=45,000$ cases analyzed in detail. Surface pressure and velocity field measurements allow for flow development within the laminar separation bubble to be appropriately described, providing context for observed trends in the surface temperature distribution. Additionally, velocity field and surface pressure measurements provide initial estimations for locations of mean separation, transition, and reattachment. Infrared thermography is employed to obtain surface temperature and convective heat transfer coefficient estimates at each flow condition. The streamwise development of surface temperature and convective heat transfer coefficient is analyzed and methodologies for estimating mean separation, transition, and reattachment locations are proposed. The estimated characteristic locations are evaluated based on their accuracy and robustness.

\subsection{Baseline Flow Measurements}

As discussed in Section 2.2.1, the location and extent of a laminar separation bubble can be determined through analysis of the surface pressure distribution. More specifically, the onset of a pressure plateau indicates separation of the boundary layer as the deadair region consists of relatively stagnant fluid [4]. This pressure plateau is followed by a region of rapid pressure recovery, with the onset of pressure recovery coinciding with 


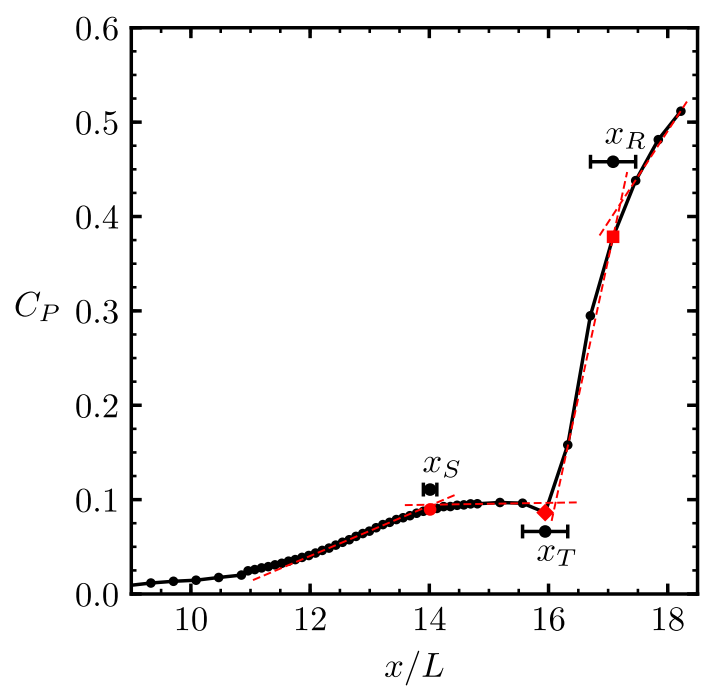

Figure 4.1: Methodology employed by Boutilier \& Yarusevych [7] for estimation of the characteristic points in a laminar separation bubble using static pressure distributions shown with an example pressure distribution $\left(\operatorname{Re}_{L}=27,000\right)$.

the mean transition location, and the end of pressure recovery marking the location of mean reattachment $[6,33]$. Due to the ambiguity associated with identifying the exact locations of separation and reattachment, a more precise methodology followed by Boutilier \& Yarusevych [7] is implemented here. An example case of this methodology is applied to $\operatorname{Re}_{L}=27,000$ in Fig. 4.1, with all highlighted Reynolds numbers shown in Fig. B.1. Lines of constant slope passing through measured pressure values are used to robustly identify the mean separation and reattachment locations, with the onset of pressure recovery used to estimate the mean transition location. The point of mean separation is located at the intersection of dashed lines upstream, and within, the pressure plateau region. The reattachment location coincides with the intersection of lines within, and downstream, of the rapid pressure recovery region. In Figs. 4.1 and B.1, and for the results presented in this thesis, the nominal locations of the characteristic points correspond to the pressure tap nearest to the appropriate intersection location, with uncertainty estimated as local pressure tap spacing.

Laminar separation bubble development is typically studied on airfoils [30, 32-34, 38$41,49]$ and flat plates with contoured bodies [9, 12, 31, 35-37, 42, 44, 46, 47, 50]. In order to confirm the fidelity of laminar separation bubble topology formed upstream of a fence on a flat plate, the measured pressure distribution was compared to inviscid solution predictions. An inviscid pressure solution was obtained using the methodology presented by Durand [116], where it is compared to measured pressures $\left(\operatorname{Re}_{L}=27,000\right)$ in Fig. 4.2. The 


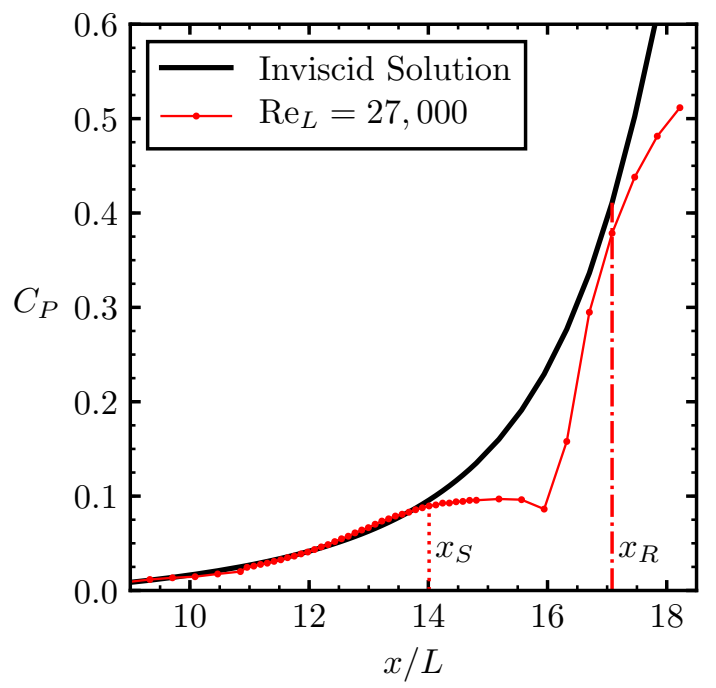

Figure 4.2: Comparison of inviscid and measured pressure distributions at $\operatorname{Re}_{L}=27,000$.

pressure distributions upstream of separation show good agreement, with deviation occurring within the separated region. Downstream of the pressure plateau, measured pressure values rapidly recover throughout the transition region and tend towards the inviscid solution at reattachment. The results suggest laminar separation bubbles, similar to those produced on airfoils and flat plates with contoured bodies, can be formed on a flat plate geometry simply with the placement of a vertical fence. Fence placement and height can be adjusted to alter the location and extent of the separated region. This approach allows for more straight-forward and repeatable results compared to other methods of imposing adverse pressure gradients on a flat plate model, such as implementation of a contoured body $[9,12,31,35-37,46,47]$ or boundary layer suction $[42,44,50]$.

Measurements of surface pressure depicted in Fig. 4.3 show the presence of a laminar separation bubble at each highlighted flow condition. The onset and completion of the pressure plateau region identifies estimated locations of mean separation and transition, shown with circle and diamond markers, respectively. The location of mean reattachment is shown at the downstream boundary of the rapid recovery region, identified with a square marker in Fig. 4.3. A summary of these estimated locations, along with their corresponding uncertainty, is shown in Table 4.1. Results from the full dataset (Table 3.1) are shown in Fig. A.1.

The results presented in Fig. 4.3 and Table 4.1 exhibit the effect of Reynolds number on the time-averaged location and extent of laminar separation bubbles. As Reynolds number is increased, the onset of boundary layer separation is delayed and the mean separation 

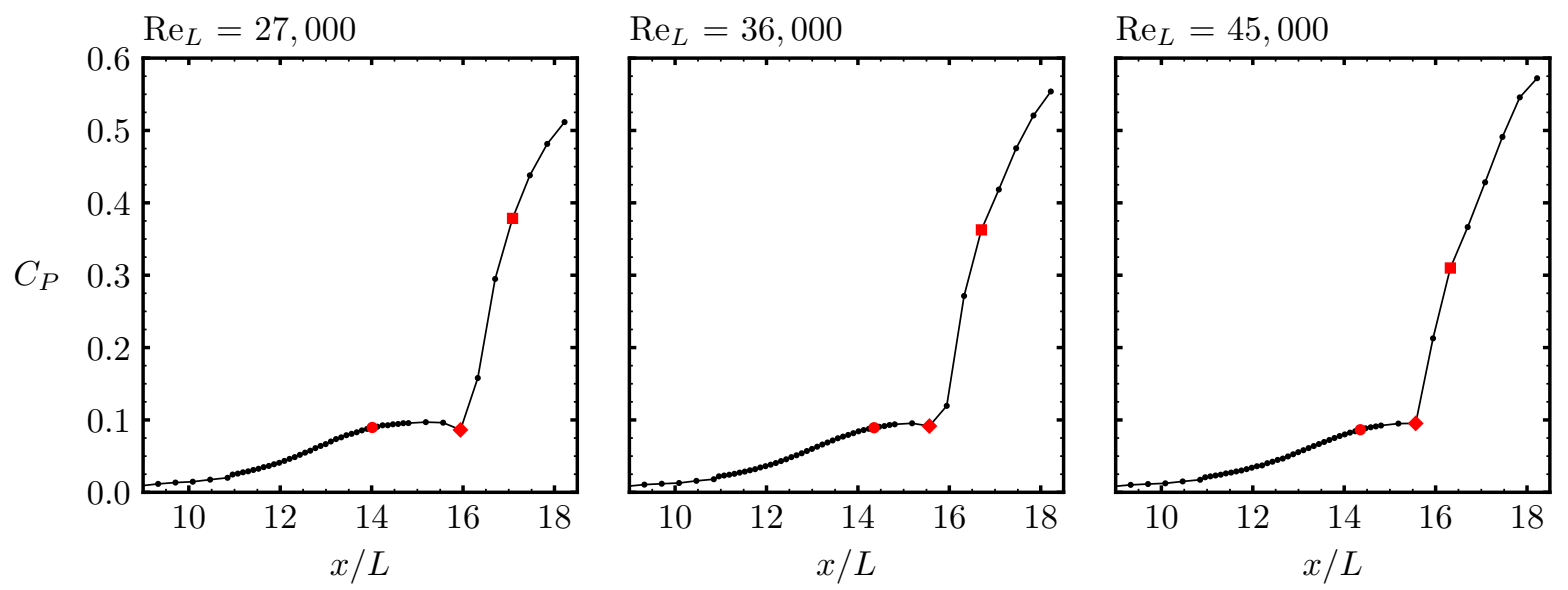

Figure 4.3: Distribution of static pressure coefficient for highlighted flow conditions. Circle, diamond, and square markers represent estimations of mean separation, transition, and reattachment, respectively.

location shifts in the downstream direction, contrary to trends shown for an airfoil geometry [7]. This suggests that the effect of increased adverse pressure gradient magnitude in the upstream flow field is less substantial than the increase of near-wall momentum from higher free-stream velocity. The near wall flow is therefore able to further withstand the adverse pressure gradient and remain attached for longer. In contrast, the mean transition and reattachment locations are shown to shift upstream as the Reynolds number is increased. A higher Reynolds number results in a more pronounced instability within the separated shear layer, due to both an increase in free-stream velocity and decrease in boundary layer thickness. Both effects result in larger wall-normal velocity gradients in the separated shear layer, which have been linked to the Kelvin Helmholtz instability mechanism [10]. Higher disturbance growth rates are therefore experienced by disturbances in the separated shear layer, promoting an earlier onset of mean transition and subsequently mean reattachment. The upstream movement of mean separation and reattachment as Reynolds number is increased results in a reduction of separation bubble extent, from $3.07 \mathrm{~L}$ at $\operatorname{Re}_{L}=27,000$, to $2.35 \mathrm{~L}$ and $1.97 \mathrm{~L}$ at $\operatorname{Re}_{L}=36,000$ and $\operatorname{Re}_{L}=45,000$, respectively.

Although measurements of surface pressure established estimations of laminar separation bubble location and extent at each flow condition, little information is provided regarding the topological characteristics of the separated region. In this regard, twocomponent, planar PIV measurements were performed, allowing for detailed analysis of flow development. Time-averaged fields of streamwise velocity are shown for highlighted Reynolds numbers in Fig. 4.4, with the reverse flow region marked with the lowest contour level. The mean dividing streamline and displacement thickness are shown with dashed and dotted lines, respectively. Since velocity measurements near the wall are prone to 


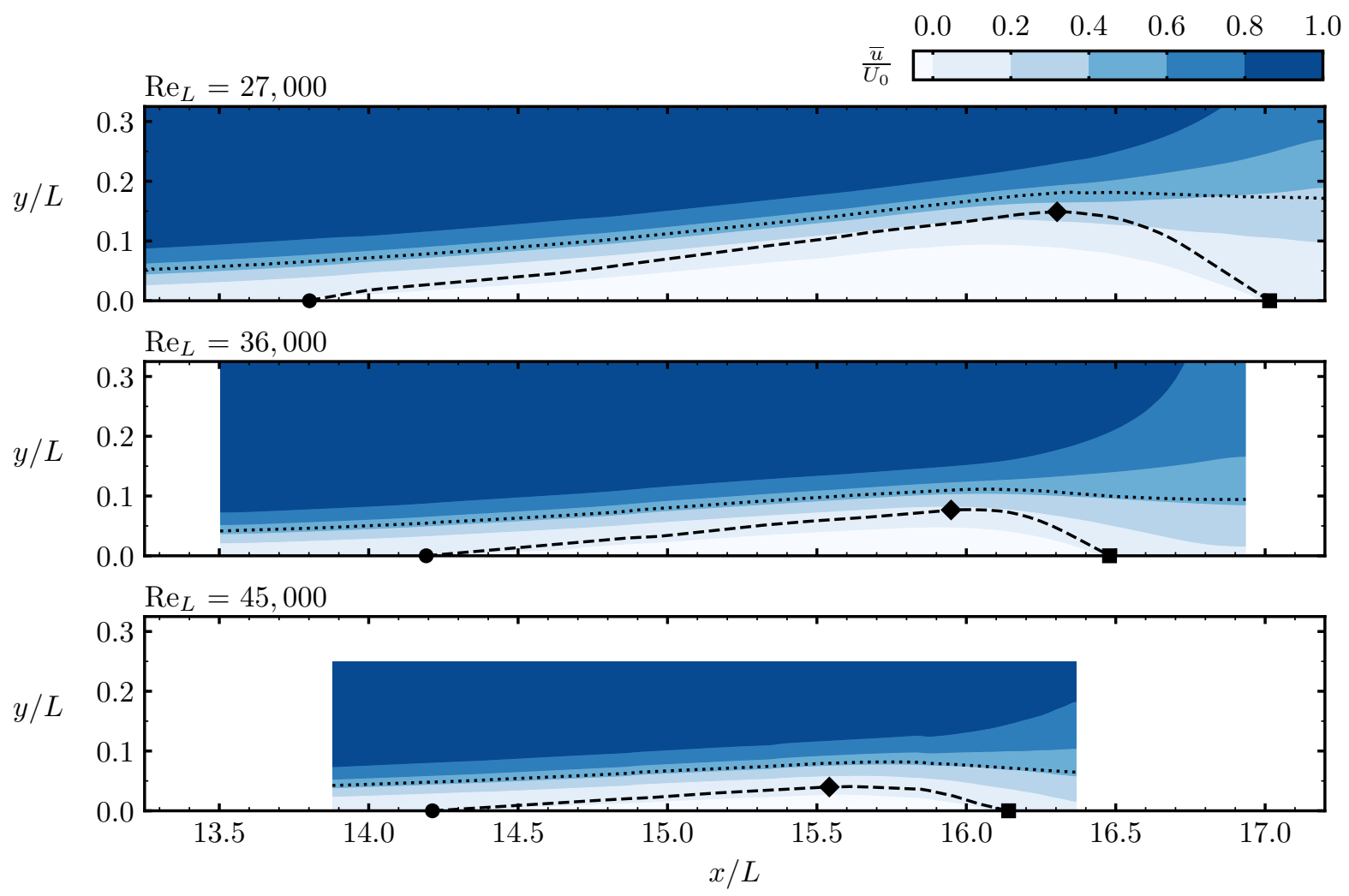

Figure 4.4: Contours of mean streamwise velocity at highlighted Reynolds numbers. Circle, diamond, and square markers denote estimated locations of separation, transition, and reattachment, respectively. Dashed and dotted lines correspond to the mean dividing streamline and displacement thickness, respectively.

higher uncertainty, a linear fit following the slope of the mean dividing streamline was extrapolated to the model surface to yield locations of mean separation and reattachment, following an approach employed in previous studies $[9,74]$. The location of mean transition can be estimated where shape factor reaches a maximum value [56, 75]. The distribution of shape factor and corresponding estimates of mean transition are shown in Fig. 4.5. The locations of mean separation, transition, and reattachment estimated from PIV measurements are indicated in Fig. 4.4 on the mean dividing streamline with circle, diamond, and square markers, respectively. Summarized estimates of these locations along with their corresponding uncertainty values are given in Table 4.1.

The time-averaged flow fields presented in Fig. 4.4 show the presence of a laminar separation bubble at each flow condition, closely resembling the topology put forth by Horton [4] (Fig. 2.2). The results exhibit downstream movement of mean separation, and upstream movement of mean transition and reattachment, as the Reynolds number 


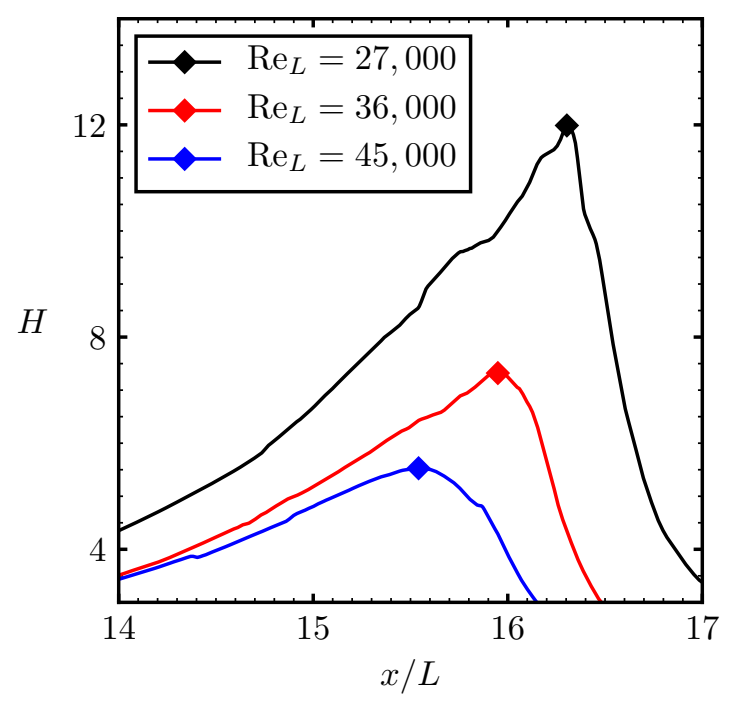

Figure 4.5: Shape factor distribution and corresponding mean transition estimates.

Table 4.1: Estimated locations of mean separation, transition, and reattachment from pressure and PIV measurements.

\begin{tabular}{lcccc}
\hline \hline Flow Condition & Measurement Technique & $x_{S} / L$ & $x_{T} / L$ & $x_{R} / L$ \\
\hline \multirow{2}{*}{$\operatorname{Re}_{L}=27,000$} & Pressure Measurements & $14.01 \pm 0.11$ & $15.94 \pm 0.38$ & $17.08 \pm 0.38$ \\
& PIV Measurements & $13.80 \pm 0.36$ & $16.30 \pm 0.03$ & $17.01 \pm 0.09$ \\
$\operatorname{Re}_{L}=36,000$ & Pressure Measurements & $14.35 \pm 0.11$ & $15.56 \pm 0.38$ & $16.70 \pm 0.38$ \\
& PIV Measurements & $14.19 \pm 0.32$ & $15.95 \pm 0.03$ & $16.48 \pm 0.05$ \\
$\operatorname{Re}_{L}=45,000$ & Pressure Measurements & $14.35 \pm 0.11$ & $15.56 \pm 0.38$ & $16.32 \pm 0.38$ \\
& PIV Measurements & $14.21 \pm 0.34$ & $15.54 \pm 0.03$ & $16.14 \pm 0.09$ \\
\hline \hline
\end{tabular}

is increased. As observed in surface pressure measurements (Fig. 4.3), this results in a reduction of separation bubble extent, from $3.21 \mathrm{~L}$ at $\mathrm{Re}_{L}=27,000$, to $2.29 \mathrm{~L}$ and $1.93 \mathrm{~L}$ at $\operatorname{Re}_{L}=36,000$ and $\operatorname{Re}_{L}=45,000$, respectively. Corresponding to the reduction in streamwise extent, the laminar separation bubble also exhibits a reduction in maximum height at higher Reynolds numbers. As such, a nearly linear trend exists between maximum displacement thickness and total streamwise extent (Fig. A.2), agreeing with observations in previous studies [33].

Comparison of the estimated mean separation, transition, and reattachment locations from pressure and PIV measurements (Table 4.1) shows overall agreement in laminar separation bubble location and extent within the bounds of uncertainty. Surface pressure measurements exhibit larger experimental uncertainty in the identification of mean transi- 
tion and reattachment locations compared to identification of the mean separation location due to the limited spatial resolution of embedded pressure taps (Fig. 3.2). Conversely, PIV estimations are associated with a larger experimental uncertainty in locating the mean separation location compared to transition and reattachment locations. This is due to the inherent difficulty of resolving low, near-wall velocities within a thin boundary layer using PIV, along with uncertainty in the exact wall location. Similar higher uncertainty bounds have been seen in previous PIV studies of laminar separation bubbles $[11,56]$

Time-averaged statistics of fluctuating velocity uncover details associated with laminar separation bubble dynamics. Contours of root-mean-square (RMS) velocity fluctuations are presented in Figs. 4.6 and 4.7 for the streamwise and wall-normal components, respectively. Estimated locations of mean separation, transition, and reattachment from PIV measurements are shown with circle, diamond, and square markers, respectively. The RMS of fluctuating velocity components (Figs. 4.6 and 4.7) exhibit trends commonly observed in laminar separation bubbles, such as the existence of peaks at a given streamwise location, particularly pronounced at, and downstream of, the maximum bubble height location $[11,56,59]$. The most prominent peak is observed in the streamwise component, at the wall-normal location of displacement thickness, marked with a dotted line. This peak extends to the fore portion of the separated region and has been attributed to shear layer flapping [74]. Towards the maximum bubble height, both streamwise and wall-normal fluctuating velocity components reach significant amplitudes earlier as the Reynolds number is increased. This is linked to the upstream movement of mean transition, as perturbations in the separated shear layer undergo stronger amplification. As shown in further detail later, this eventually leads to shear layer roll-up into vortices near mean transition. The inherently unsteady roll-up and shedding of vortices induces significant velocity fluctuations, which increase throughout the transition region. Shed vortices follow a trajectory aimed at the surface, resulting in periodic flow impingement and subsequent vortex breakdown in the reattachment region. The reattaching flow is therefore associated with significantly increased momentum exchange, as shown with contours of Reynolds shear stress (RSS) in Fig. 4.8.

Dynamics of the laminar separation bubble associated with shear layer vortex shedding are shown in Fig. 4.9 with contours of instantaneous spanwise vorticity. FOV 4 shows the formation and evolution of a vortex over several sequential vorticity fields. The vortex entrains fluid from the outer flow, eventually recovering enough streamwise momentum to shed from the separated shear layer. As the shed vortex convects downstream (Fig. 4.9(FOV 5)), it partially impinges on the surface, entrains induced near-wall vorticity, and breaks down to smaller-scale structures. Vortex impingement results in significant velocity fluctuations and RSS, causing maximum values to be attained in the vicinity of 


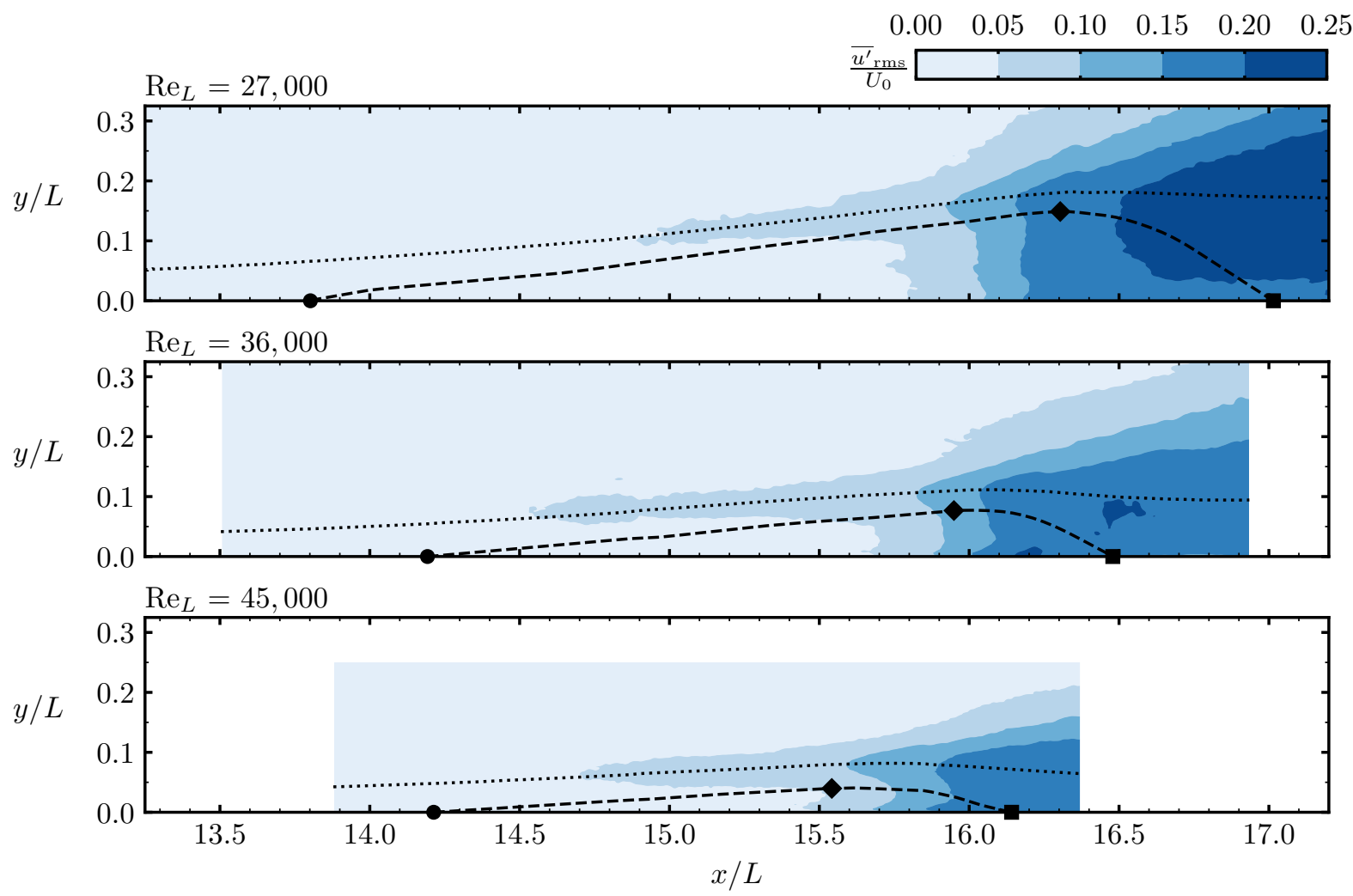

Figure 4.6: Contours of the streamwise RMS component of fluctuating velocity. Circle, diamond, and square markers denote estimated locations of separation, transition, and reattachment, respectively. Dashed and dotted lines correspond to the mean dividing streamline and displacement thickness, respectively.

mean reattachment(Figs. 4.6-4.8).

Streamwise flow development shown in Figs. 4.4 and $4.6-4.8$ can be used to gain insight into expected trends in convective heat transfer and surface temperature beneath the laminar separation bubble. In the case of elevated wall temperature facilitated in the recent study, deceleration of the near-wall flow, boundary layer separation, and nearstagnant fluid in the fore portion of the bubble are expected to hinder local convective heat transfer and therefore increase surface temperature. In contrast, the amplification of velocity fluctuations associated with transition, and the periodic vortex impingement associated with reattachment are expected to significantly increase local convective heat transfer, reducing temperature at the surface. The following section (Section 4.2) presents time-averaged surface temperature distributions, and corresponding estimates of the convective heat transfer coefficient. These distributions are related to observations in velocity and pressure measurements in order to characterize near-wall flow behaviour. 


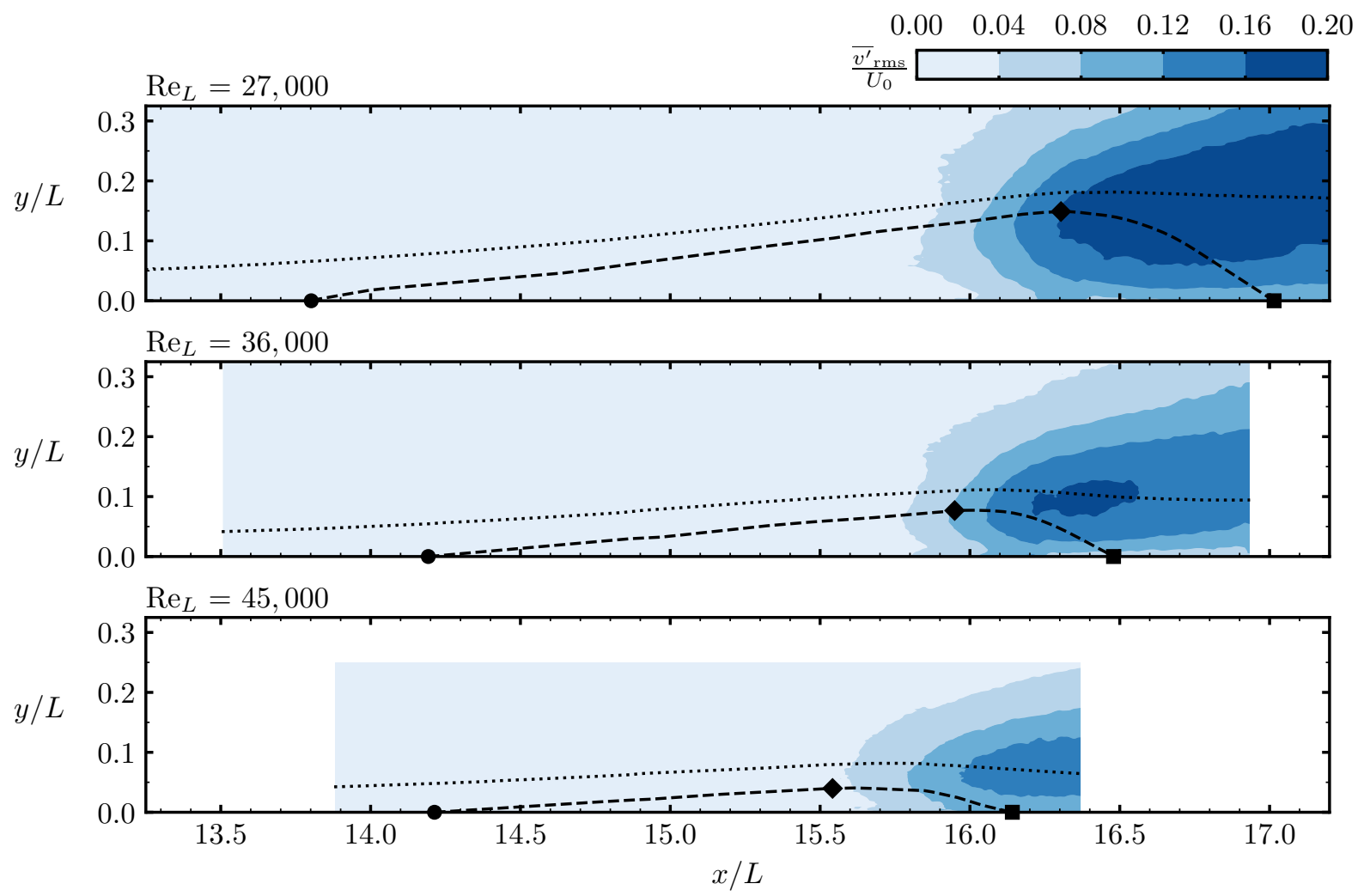

Figure 4.7: Contours of the wall-normal RMS component of fluctuating velocity. Circle, diamond, and square markers denote estimated locations of separation, transition, and reattachment, respectively. Dashed and dotted lines correspond to the mean dividing streamline and displacement thickness, respectively.

\subsection{Time-Averaged Thermal Analysis}

Results presented in Section 4.1 put forward an overview of the spatio-temporal flow development, and overall flow topology, associated with a laminar separation bubble formed on a flat plate upstream of a vertically mounted fence. Initial estimates for the location and extent of the separated region are provided though analysis of surface pressure and PIV measurements. Additionally, a brief overview of expected trends in surface temperature and convective heat transfer distributions are presented. In this section, time-averaged surface temperature distributions obtained with infrared thermography are presented for highlighted flow conditions. Additionally, surface temperature distributions are used to characterize convective heat transfer at the plate surface. Analysis of the surface temperature and convective heat transfer coefficient distribution is performed and related to trends expected from PIV and surface pressure measurements. Methodologies for char- 


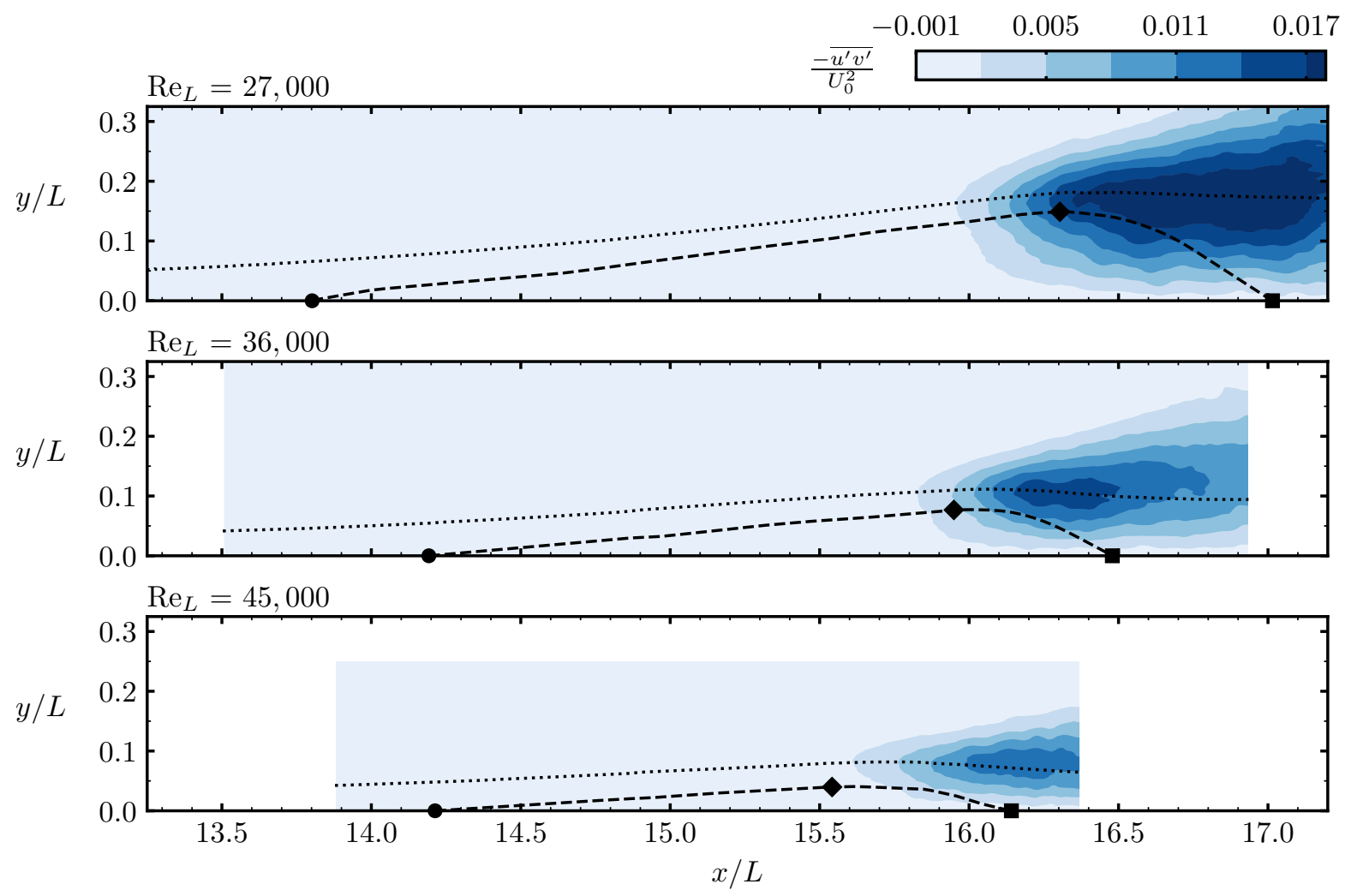

Figure 4.8: Reynolds shear stress contours. Circle, diamond, and square markers denote estimated locations of separation, transition, and reattachment, respectively. Dashed and dotted lines correspond to the mean dividing streamline and displacement thickness, respectively.

acterizing the separated region based on surface temperature measurements are proposed and evaluated based on their accuracy and robustness.

Infrared thermography allows for surface temperature distributions on the flat plate to be attained. Contours of surface temperature in the field of view defined in Fig. 3.2b are shown for highlighted flow conditions in Fig. 4.10. The contour levels are shown in $0.5^{\circ} \mathrm{C}$ increments, with PIV estimations of mean separation, transition, and reattachment shown with dotted, dashed, and dash-dotted lines, respectively. The temperature fields are time-averaged, and spatially filtered with a 5px kernel to mitigate spurious artifacts in the temperature distribution. An additional 5px spatial filter is applied to all presented spanwise-averaged distributions, ultimately allowing for a clearer representation of surface temperature development.

Surface temperature contours shown in Fig. 4.10 exhibit a band of increased temperature within the region associated with a laminar separation bubble at all Reynolds numbers 

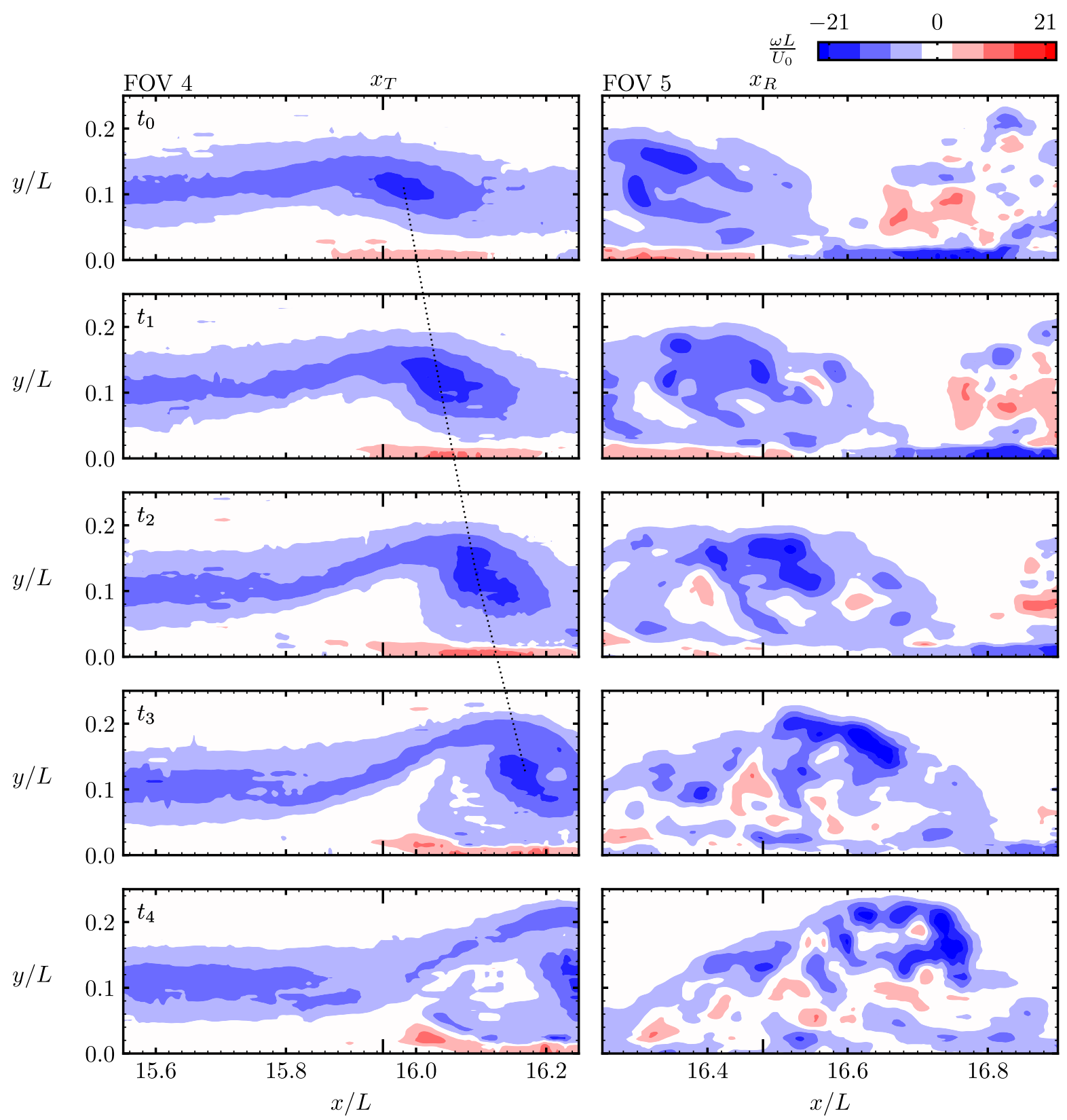

Figure 4.9: Instantaneous contours of spanwise vorticity at $\operatorname{Re}_{L}=36,000$. Two independent fields of view are shown corresponding to the fourth and fifth field of view in Fig. 3.2. $\Delta t$ between frames is $1 \mathrm{~ms}$ such that one complete cycle of vortex shedding is captured. Estimations of mean transition and reattachment are shown with extended tick markers. 
presented. The location of maximum surface temperature shifts slightly downstream with increased Reynolds number, while the downstream edge of the elevated surface temperature band shifts in the upstream direction. Overall, these effects result in a reduction in streamwise extent of the elevated surface temperature band, agreeing with trends associated with laminar separation bubbles arising from increases in Reynolds number (Table 4.1) [7, 10]. The increased surface temperature band location observed in Fig. 4.10 supports the observations of Montelpare \& Ricci [27], who identified laminar separation bubbles on an airfoil with the presence of a spanwise band of elevated surface temperature.

The surface temperature development observed in Fig. 4.10 can be interpreted with consideration of the known near-wall flow behaviour. Upstream of mean separation, due to growth of the attached boundary layer, the convective heat transfer coefficient is expected to gradually decrease in the streamwise direction (Eq. 2.7), therefore resulting in a gradually increasing surface temperature (Eq. 2.1). This behaviour is expected to be amplified due to the deceleration of near-wall flow caused by the presence of an adverse pressure gradient [107]. Slow moving fluid in the fore portion of the separation bubble is associated with minimal convective heat transfer intensity, and consequently a maximum surface temperature $[34,114]$. The onset of transition causes rapid intensification of convective heat transfer, and a corresponding reduction in surface temperature, downstream of the absolute maximum temperature location. This is due to a significant increase in fluctuating velocity and momentum exchange in the near-wall region. Periodic impingement of shear layer vortices near mean reattachment results in minimum surface temperature, and therefore maximum levels of convective cooling. Flow in the vicinity of mean reattachment behaves similar to impinging jets [117] and other separating-reattaching flows [118], which experience a maximum convective heat transfer coefficient in the reattaching region. Downstream of mean reattachment, a gradual increase in surface temperature, and decrease in convective heat transfer coefficient, is expected due to turbulent boundary layer development (Eq. 2.8). The rate of temperature increase in this region however is less notable than in attached laminar flow (Fig. 4.10 and Eqs. (2.7) and (2.8)).

Although the infrared field of view was not centered about the mid-span of the flat plate (Fig. 3.2b), it is demonstrated in Fig. 4.10 that reasonable spanwise uniformity of surface temperature was obtained for all Reynolds numbers. This suggests that the spanwise-averaged distribution of mean surface temperature can be used to represent the time-average surface temperature distribution. Distributions of spanwise-averaged surface temperature, and corresponding streamwise temperature gradients, are presented in Fig. 4.11. Such distributions allow for a more quantitative analysis of convective heat transfer trends, such that the locations of mean separation, transition, and reattachment can be estimated. The measured ambient temperature, $T_{\infty}$, is subtracted from the surface tem- 


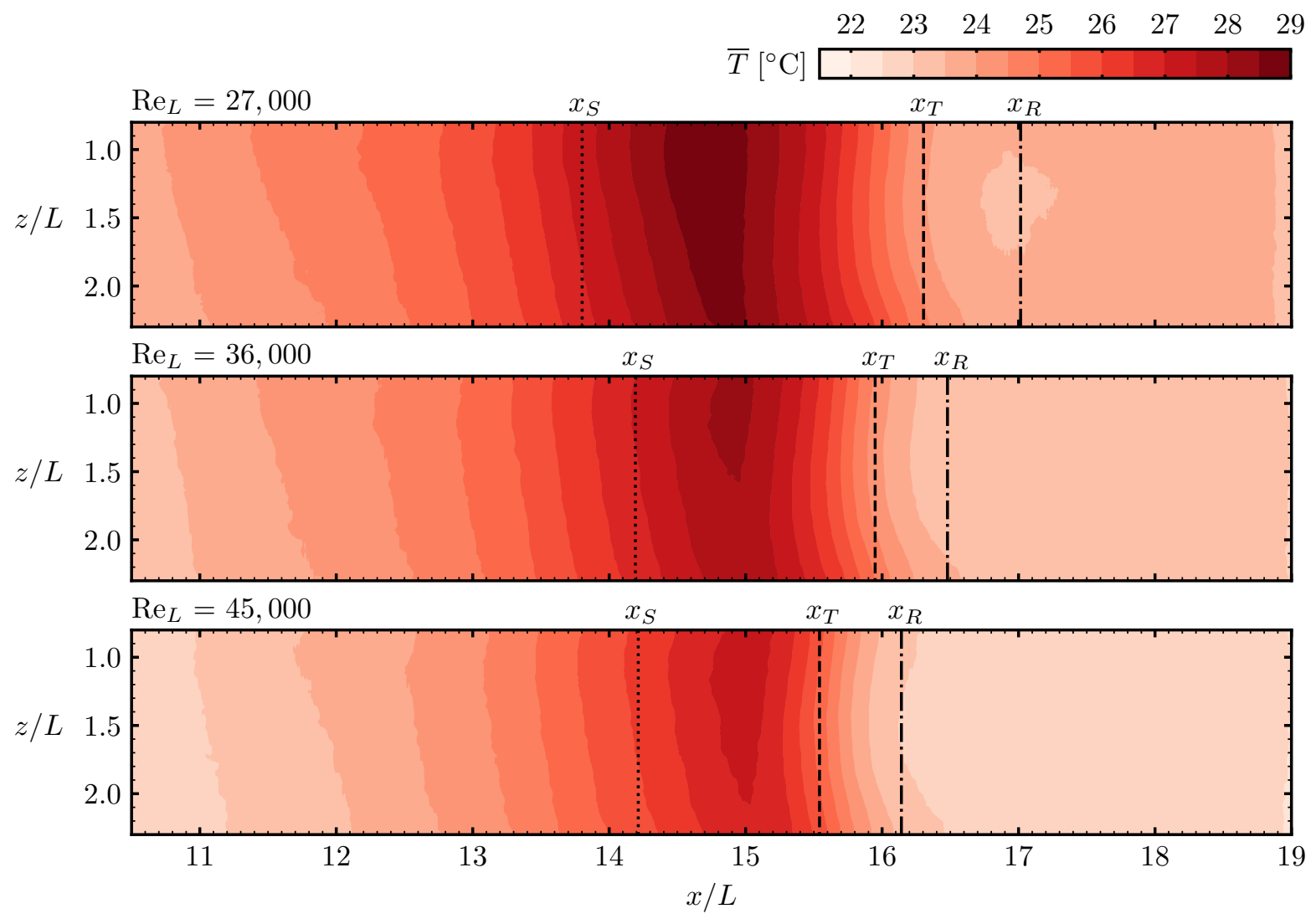

Figure 4.10: Contours of time-averaged temperature measured on the flat plate surface. Estimations of mean separation, transition, and reattachment from PIV measurements are shown with dotted, dashed, and dash-dot lines, respectively.

perature in Fig. 4.11, with $\left(\bar{T}-T_{\infty}\right)$ serving as an approximation of the inverse convective heat transfer coefficient (Eq. 2.1). Using the theoretical predictions introduced in Section 3.3, and the methodology discussed below, locations of mean separation, transition, and reattachment are estimated from the temperature distributions shown in Fig. 4.11, where they are identified with circle, diamond, and square markers, respectively. Supplementary results containing surface temperature distributions and corresponding streamwise temperature gradients from the full dataset (Table 3.3) are shown in Fig. A.4.

Spanwise-averaged distributions of surface temperature allow for quantitative characterization of the laminar separation bubble. Upstream of mean separation, the surface temperature, and corresponding streamwise temperature gradient, increase due to growth of the attached laminar boundary layer and near-wall flow deceleration. The temperature gradient attains a maximum value near the point of mean separation, where a minimum gradient of convective heat transfer coefficient has been observed in convection dominant flows [114]. Downstream of separation, the streamwise gradient of surface temperature de- 


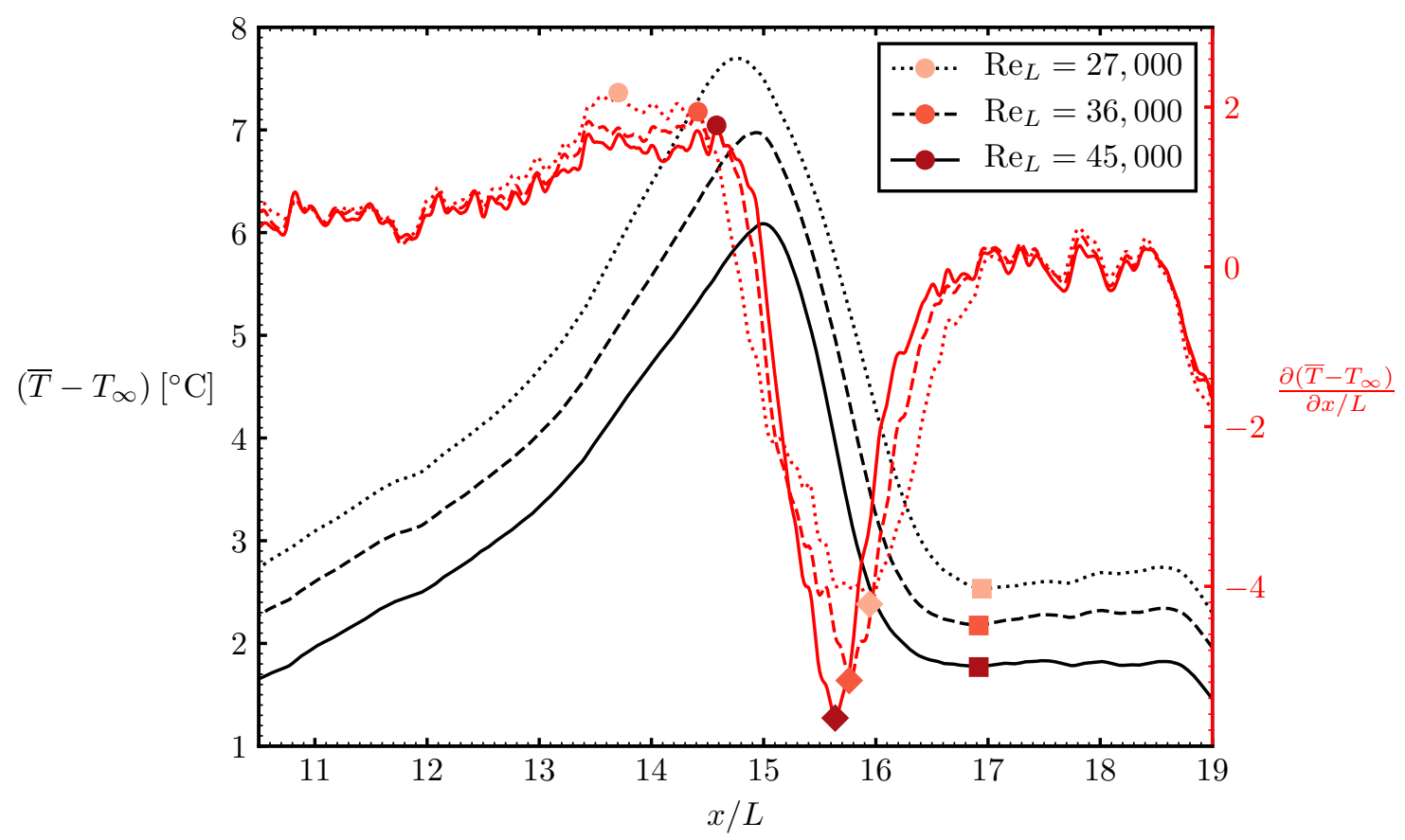

Figure 4.11: Time- and spanwise-averaged distributions of surface temperature and its streamwise gradient. Estimated locations of mean separation, transition, and reattachment are marked with circle, diamond, and square markers, respectively.

creases due to the upstream movement of cool fluid entrained during the transition process. Therefore, the point of mean separation can be estimated at the location where the streamwise gradient of surface temperature attains a maximum value. This is supported by DNS results of Spalart \& Strelets [114], where the location of separation is shown to coincide with the steepest decrease of convective heat transfer coefficient. The surface temperature achieves a maximum value within the fore portion of the separated region, before rapidly decreasing with the onset of transition. The location of maximum surface temperature is expected to coincide with the minimum convective heat transfer coefficient, which has been shown to occur in the fore portion of separation bubbles in previous studies $[34,46,114]$. Downstream of the maximum temperature location, significant increases in fluctuating velocity and fluid entrainment rapidly increase the convective heat transfer coefficient [114], consequently resulting in a rapid decrease of surface temperature Fig. 4.11. Following an approach employed in previous studies of near-wall transition prediction [19,20, 24, 8285], the location of mean transition is estimated where the streamwise gradient of surface temperature achieves a minimum value.

Temperature at the surface is further reduced downstream of mean transition, achieving a minimum value in the vicinity of mean reattachment. Periodic vortex impingement 
and amplified levels of fluctuating velocity in the reattaching region result in a maximum convective heat transfer coefficient $[34,114,117]$. However, disagreement persists regarding the exact location of mean reattachment in relation to the point of maximum convective heat transfer in reattaching flows. For example, Pucher \& Gohl [34] found the reattachment location to lie just upstream of the maximum convective heat transfer coefficient location when comparing thermal measurements from heated thin-film sensors to measurements of surface pressure on an airfoil. Conversely, Spalart \& Strelets [114] show the reattachment location to lie slightly downstream of the maximum convective heat transfer location in a numerical study of laminar separation bubbles on a flat plate, with others suggesting the reattachment location coincides with the location of maximum convective heat transfer [28, 118-120]. Following the justification introduced in Section 3.3, it is proposed here that the mean reattachment location can be estimated where surface temperature reaches a local minimum, downstream of the increased temperature band. This location approximately corresponds to a maximum in the convective heat transfer coefficient for flows where forced convection is the dominant mode of heat transfer (Eq. 2.1). Characterization of mean reattachment employing this methodology allows for a robust estimation which does not rely on ad-hoc thresholding to slightly offset mean reattachment from the location of maximum convective heat transfer coefficient or minimum surface temperature.

Following the methodology presented above, estimated locations of mean separation, transition, and reattachment are identified in Fig. 4.11, with a summary of their locations and associated uncertainty values provided in Table 4.2. The location of mean separation is shown to shift in the downstream direction as the Reynolds number is increased, agreeing within uncertainty bounds with the results from PIV and surface pressure data at all Reynolds numbers. However, it should be noted that the location of mean separation determined with distributions of surface temperature is estimated at a weakly defined peak value of streamwise temperature gradient. This introduces relatively large methodological uncertainty as the identification is performed within a region of relatively constant temperature gradient, resulting in an identification which is sensitive to small spatial fluctuations in surface temperature. The location of mean transition estimated from the surface temperature gradient agrees well with estimations provided by surface pressure and PIV measurements. Mean transition estimates are located at a well-defined maximum gradient value which remains relatively unchanged across the span, resulting in estimations which are associated with relatively low methodological uncertainty. Estimations of mean reattachment from surface temperature distributions agree with those from PIV and pressure measurements at $\operatorname{Re}_{L}=27,000$. However, temperature-based estimates deviate more at $\operatorname{Re}_{L}=36,000$ and $\operatorname{Re}_{L}=45,000$, where the estimated location of mean reattachment remains effectively unchanged as the Reynolds number is increased. This is due to a temperature plateau experienced in the aft portion of the separation bubble (Fig. 4.11), resulting in a weakly 


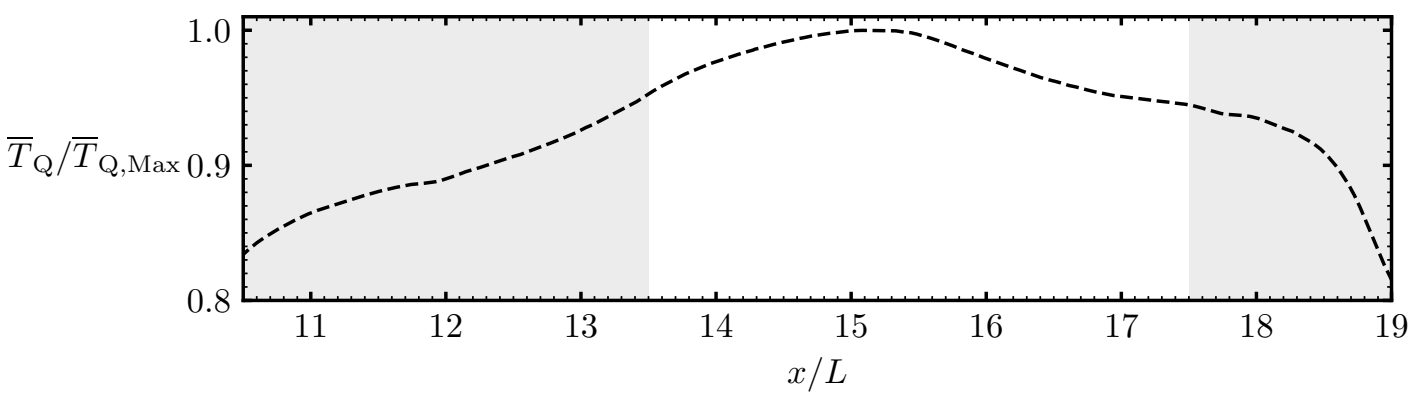

Figure 4.12: Time- and spanwise-averaged distribution of normalized quiescent surface temperature. Unshaded area represents the general region where laminar separation bubbles were observed in this study.

defined location of minimum surface temperature. Mean reattachment estimations from surface temperature measurements are therefore vulnerable to small spatial fluctuations in surface temperature resulting in a less precise characterization.

An apparent issue with the proposed methodology of characterizing laminar separation bubbles using distributions of surface temperature is the validity of assuming constant radiative heat flux at the surface, and negligible, or constant, conductive heat flux within the model, as both assumptions affect initial uniformity of surface temperature. Although careful attention was paid to placement of the halogen lamps in order to ensure uniform radiative flux at the surface, uniform surface heating is difficult to achieve in practice without the use of specialized surface heating elements. Moreover, the wall-normal temperature gradient through the flat plate model and the aluminium sections present on both sides of the Lexan centerpiece (Fig. 3.2a) inherently affect heat conduction intensity in the wall-normal and streamwise directions, respectively. The effect of non-uniform model heat flux can be visualized in the distribution of surface temperature in quiescent conditions, as shown in Fig. 4.12. The results exhibit a non-uniform surface temperature, varying by approximately $5 \%$ within the region associated with the laminar separation bubble, where a maximum quiescent temperature is achieved approximately in the same location as that with forced convection (Fig. 4.10).

While the observed non-uniformities in the quiescent temperature distribution are not substantial, it is of interest to account for them, particularly in situations where larger variations are observed, in order to yield a more accurate characterization of the laminar separation bubble. For a flat plate heated to thermal equilibrium with negligible conduction, the surface heat flux in quiescent conditions per unit area can be written as:

$$
q_{\text {Rad }}^{\prime \prime}=h_{\text {Free }}\left(\bar{T}_{Q}-T_{\infty}\right)
$$


Similarly, for a heated model in thermal equilibrium which is subject to forced convection, the surface heat flux per unit area can be written as:

$$
q_{\mathrm{Rad}}^{\prime \prime}=h\left(\bar{T}-T_{\infty}\right)
$$

Equating Eqs. (4.1) and (4.2) and rearranging, a normalized local convective heat transfer coefficient, $h^{*}$, can be obtained as follows:

$$
\frac{h}{h_{\text {Free }}}=h^{*}=\frac{\left(\bar{T}_{Q}-T_{\infty}\right)}{\left(\bar{T}-T_{\infty}\right)}
$$

Since $h_{\text {Free }}$ is expected to be approximately constant along the horizontal flat plate, Eq. 4.3 accounts for non-uniformities in incident heating with use of the quiescent temperature distribution. Equation 4.3 is applied at every pixel location on the flat plate to compute $h^{*}(x, z)$, which is then averaged across the span $(z)$, yielding an estimated distribution of normalized convective heat transfer coefficient along the streamwise direction. Such distributions can be used to obtain estimates of mean separation, transition, and reattachment locations using the methodology established above. More specifically, the locations of mean separation and transition can be estimated where the streamwise gradient of the convective heat transfer coefficient reaches minimum and maximum values, respectively. The location of mean reattachment can be estimated where a maximum convective heat transfer coefficient is observed. Distributions of $h^{*}$ and its corresponding streamwise gradients are shown for each highlighted Reynolds number in Fig. 4.13. Estimated locations of mean separation, transition, and reattachment are identified with circle, diamond, and square markers, respectively. A summary of these locations with corresponding uncertainty estimates is shown in Table 4.2.

The streamwise distributions of $h^{*}$ shown in Fig. 4.13 follow expected trends of convective heat transfer beneath a laminar separation bubble. A reduction in convective heat transfer coefficient is observed upstream of mean separation, with a minimum value occurring in the fore portion of the separated region. However, a nearly linear decrease of $h^{*}$ is observed upstream of the absolute minimum, consequently making it difficult to estimate mean separation at the minimum streamwise gradient of $h^{*}$. Therefore, without significant loss of accuracy, the mean separation location is identified at the intersection of linear lines passing through the regions of plateau and subsequent rapid increase of $\frac{\partial h^{*} / h_{\text {Min }}^{*}}{\partial x / L}$, as shown in Fig. B.2. The rapid increase in both $h^{*}$ and its streamwise gradient downstream of this identified location is due to both the increase in reverse flow magnitude, and the rapid onset of transition within the separated region. The value of $h^{*}$ reaches a maximum in the reattaching region, as observed in other separating-reattaching flows [118]. This is followed by a gradual decrease in $h^{*}$ due to development of the attached turbulent boundary layer. 


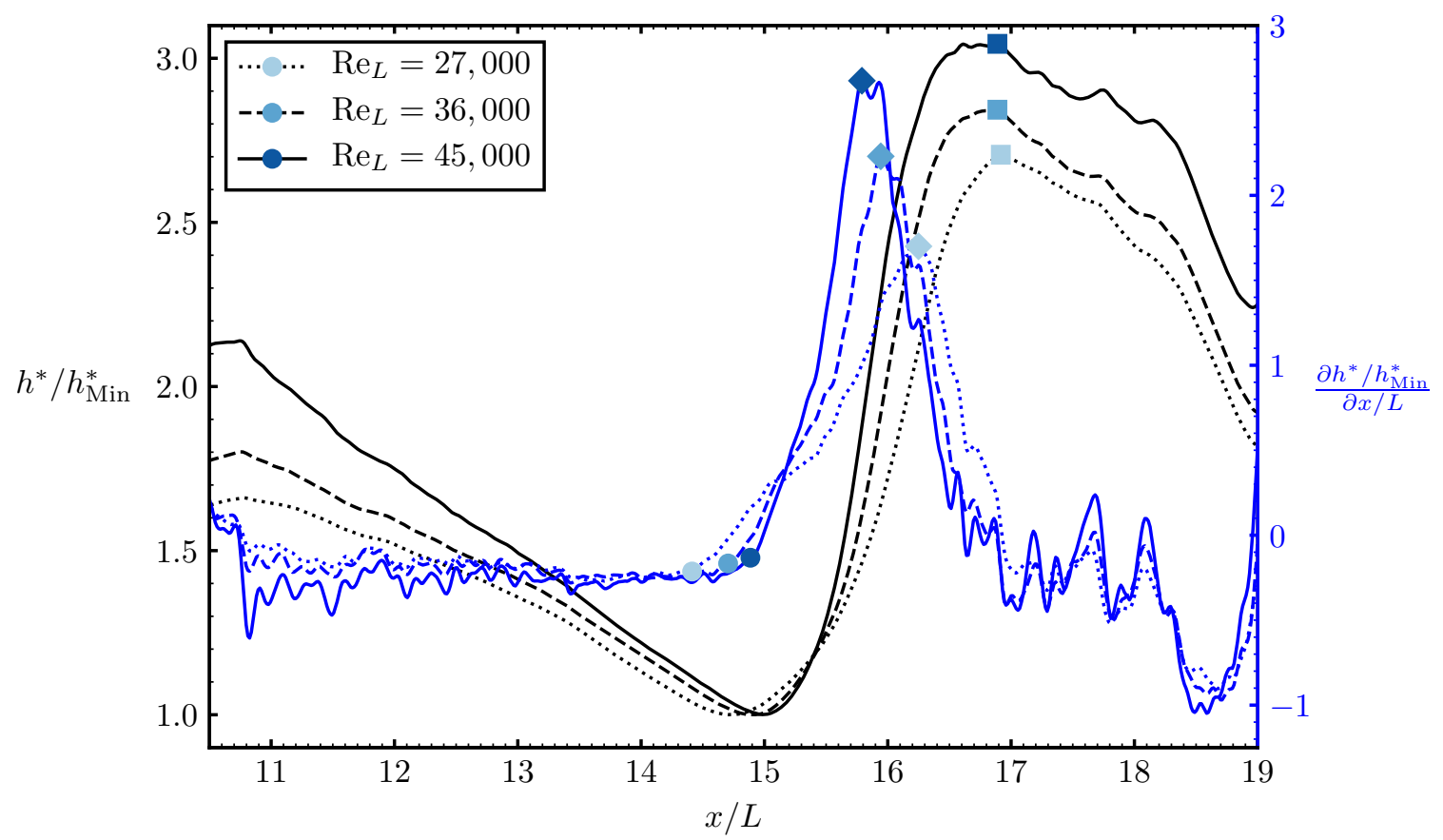

Figure 4.13: Time- and spanwise-averaged distributions of $h^{*}$ and its streamwise gradient. Estimated locations of mean separation, transition, and reattachment are identified with circle, diamond, and square markers, respectively.

The locations of mean separation, transition, and reattachment estimated from distributions of $h^{*}$ overall exhibit reasonable agreement with those estimated from PIV and surface pressure measurements. Although the locations of mean separation estimated from distributions of $h^{*}$ are shown to agree within the bounds of uncertainty associated with PIV estimations, the identified mean separation locations appear to be biased in the downstream direction. This is likely due to uncertainty associated with locating the minimum streamwise gradient of $h^{*}$, as a region of relatively constant slope is observed near mean separation (Fig. 4.13). The location of mean transition agrees with estimations from PIV and pressure measurements for each Reynolds number, and is identified at a distinct peak location of the streamwise gradient of $h^{*}$. Peak values of $h^{*}$ used to identify mean reattachment produce estimates which agree reasonably well with PIV and surface pressure estimations at $\operatorname{Re}_{L}=27,000$; however, there may be a slight downstream bias in mean reattachment location estimates at $\operatorname{Re}_{L}=36,000$ and $\operatorname{Re}_{L}=45,000$. This can be attributed to the relatively shallow peak observed in the distribution of $h^{*}$ near mean reattachment, such that the estimated location is susceptible to small spatial variations in measured surface temperature.

The validity of assuming negligible conductive heat flux within the flat plate model is 
evaluated in Appendix C. Through a complete differential energy balance at the surface and within the model, considering heat flux through free and forced convection, streamwise and wall-normal conduction, as well as incident and outgoing radiation, the dominating modes of heat transfer become apparent. It is shown that negligible streamwise conduction can be reasonably assumed due to the insignificant difference in local streamwise temperature gradient between quiescent and flow conditions, and low thermal conductivity of the model material. Heat flux in the wall-normal direction occurs due to conduction within the model and convection at the bottom surface, while radiative cooling occurs due to the temperature difference between the model surface and the surroundings. Both downward heat flux and radiative cooling are shown to exhibit the same streamwise variation as that of forced convection, with radiative heat loss being of a comparable magnitude to that of forced convective heat loss at the top surface. In order to accurately determine the nominal value of convective heat transfer coefficient, these heat fluxes must be considered. However, it is shown that the forced convection at the top surface, the wall-normal heat flux, and the heat flux through radiative cooling are all dependent on $O\left(\frac{\left(\bar{T}_{Q}-T_{\infty}\right)}{\left(\bar{T}-T_{\infty}\right)}\right)$, which appears in the expression for $h^{*}$ (Eq. 4.3). Therefore, locations where extrema are observed in distributions of $h^{*}$ are effectively unchanged with consideration of the downward and radiative heat losses, which is the main interest of the proposed methodology.

The effect of considering, and partially correcting for, non-uniform surface heating becomes evident when comparing estimated locations of mean separation, transition, and reattachment established with distributions of surface temperature and $h^{*}$ (Table 4.2). Although both methodologies estimate mean separation locations which agree within uncertainty bounds of those from surface pressure and PIV measurements, the uncertainty associated with such estimations is relatively large. This can be attributed to the measurement noise propagation to the streamwise gradient distributions, used for the estimation of mean separation. As a consequence, mean separation estimates show increased sensitivity to minute thermal fluctuations. The locations of mean transition estimated from both methodologies are also shown to agree well with estimations from PIV and surface pressure measurements. This is due to substantial gradients of temperature and convective heat transfer coefficient present within the transition region, allowing for an accurate and robust identification, a result which has been observed in previous studies $[19,20,24,82-$ 85]. Although the identified location of mean reattachment is effectively unchanged when estimated from distributions of surface temperature and $h^{*}$, a less substantial plateau is observed in the reattachment region with distributions of $h^{*}$. This highlights the importance of considering non-uniformities in radiative and conductive heat flux, as these effects cause distributions of mean surface temperature to experience a more significant plateau near mean reattachment, resulting in less precise estimations associated with higher methodological uncertainty. 
Table 4.2: Estimated locations of mean separation, transition, and reattachment on the flat plate from each measurement technique.

\begin{tabular}{ccccc}
\hline \hline Flow Condition & Measurement Technique & $x_{S} / L$ & $x_{T} / L$ & $x_{R} / L$ \\
\hline & Pressure Measurements & $14.01 \pm 0.11$ & $15.94 \pm 0.38$ & $17.08 \pm 0.38$ \\
$\operatorname{Re}_{L}=27,000$ & PIV Measurements & $13.80 \pm 0.36$ & $16.30 \pm 0.03$ & $17.01 \pm 0.09$ \\
& $\bar{T}$ Distribution & $13.70_{-0.83}^{+0.92}$ & $15.94_{-0.49}^{+0.28}$ & $16.94_{-0.14}^{+0.35}$ \\
& $h^{*}$ Distribution & $14.41_{-0.67}^{+0.60}$ & $16.25_{-0.24}^{+0.17}$ & $16.92_{-0.09}^{+0.11}$ \\
& Pressure Measurements & $14.35 \pm 0.11$ & $15.56 \pm 0.38$ & $16.70 \pm 0.38$ \\
$\operatorname{Re}_{L}=36,000$ & PIV Measurements & $14.19 \pm 0.32$ & $15.95 \pm 0.03$ & $16.48 \pm 0.05$ \\
& $\bar{T}$ Distribution & $14.41_{-1.52}^{+0.42}$ & $15.76_{-0.20}^{+0.24}$ & $16.92_{-0.22}^{+0.16}$ \\
& $h^{*}$ Distribution & $14.71_{-0.56}^{+0.55}$ & $15.94_{-0.14}^{+0.20}$ & $16.89_{-0.10}^{+0.10}$ \\
& Pressure Measurements & $14.35 \pm 0.11$ & $15.56 \pm 0.38$ & $16.32 \pm 0.38$ \\
$\operatorname{Re}_{L}=45,000$ & PIV Measurements & $14.21 \pm 0.34$ & $15.54 \pm 0.03$ & $16.14 \pm 0.09$ \\
& $\bar{T}$ Distribution & $14.58_{-2.05}^{+0.38}$ & $15.64_{-0.21}^{+0.20}$ & $16.92_{-0.32}^{+0.34}$ \\
& $h^{*}$ Distribution & $14.89_{-0.55}^{+0.54}$ & $15.79_{-0.15}^{+0.24}$ & $16.89_{-0.43}^{+0.10}$ \\
\hline \hline
\end{tabular}

The results presented in this chapter demonstrate the feasibility of using infrared thermography to identify the location, size, and extent of laminar separation bubbles formed on a flat plate. Through the analysis of surface temperature, estimations of mean separation, transition, and reattachment can be established. Effects of non-uniform radiative heat flux and non-zero conductive heat flux were partially compensated for with consideration of quiescent temperature distributions and the attendant heat transfer. The distribution of normalized convective heat transfer coefficient was estimated and used to locate characteristic points of the laminar separation bubble. The results obtained from distributions of both surface temperature and convective heat transfer coefficient generally agree with estimations from PIV and surface pressure measurements. However, the mean reattachment location was shown to be relatively unchanged as Reynolds number was increased for both methodologies. This can be attributed to the plateau region observed in the vicinity of reattachment, which was shown to be more significant in distributions of surface temperature. The following chapter applies the proposed methodology to an airfoil geometry, providing further analysis of thermal behaviour beneath laminar separation bubbles. 


\section{Chapter 5}

\section{Streamwise Flow Development of a Laminar Separation Bubble on a NACA 0018 Airfoil}

In this chapter, laminar separation bubbles formed on a NACA 0018 airfoil are investigated in terms of their spatio-temporal development and corresponding thermal signature. Measurements presented pertain to an angle of attack of $4^{\circ}$ and two chord-based Reynolds numbers: $\operatorname{Re}_{c}=80,000$ and $\operatorname{Re}_{c}=120,000$, with supplementary results from the full dataset (Table 3.2) shown in Appendix A. These conditions were selected as they produce typical short laminar separation bubbles previously observed on various airfoil geometries [7, 38, 81, 121]. Two-component, planar PIV measurements were performed in order to analyze laminar separation bubble development, and estimate the corresponding location and extent. Additionally, PIV measurements provide context for observed behaviour in surface temperature distributions. Infrared thermography was employed, allowing for analysis of the surface temperature and convective heat transfer coefficient within the separated region. The laminar separation bubble characterization methodologies proposed in Chapter 4 are applied and the corresponding characteristic locations are compared to those obtained from PIV measurements. Additionally, distributions of the convective heat transfer coefficient are estimated using transient surface temperature measurements. The distribution of convective heat transfer coefficient is used to characterize the location and extent of the separated region, and the proposed methodologies are compared to one another in terms of their accuracy and robustness, with PIV estimations serving as a baseline. 


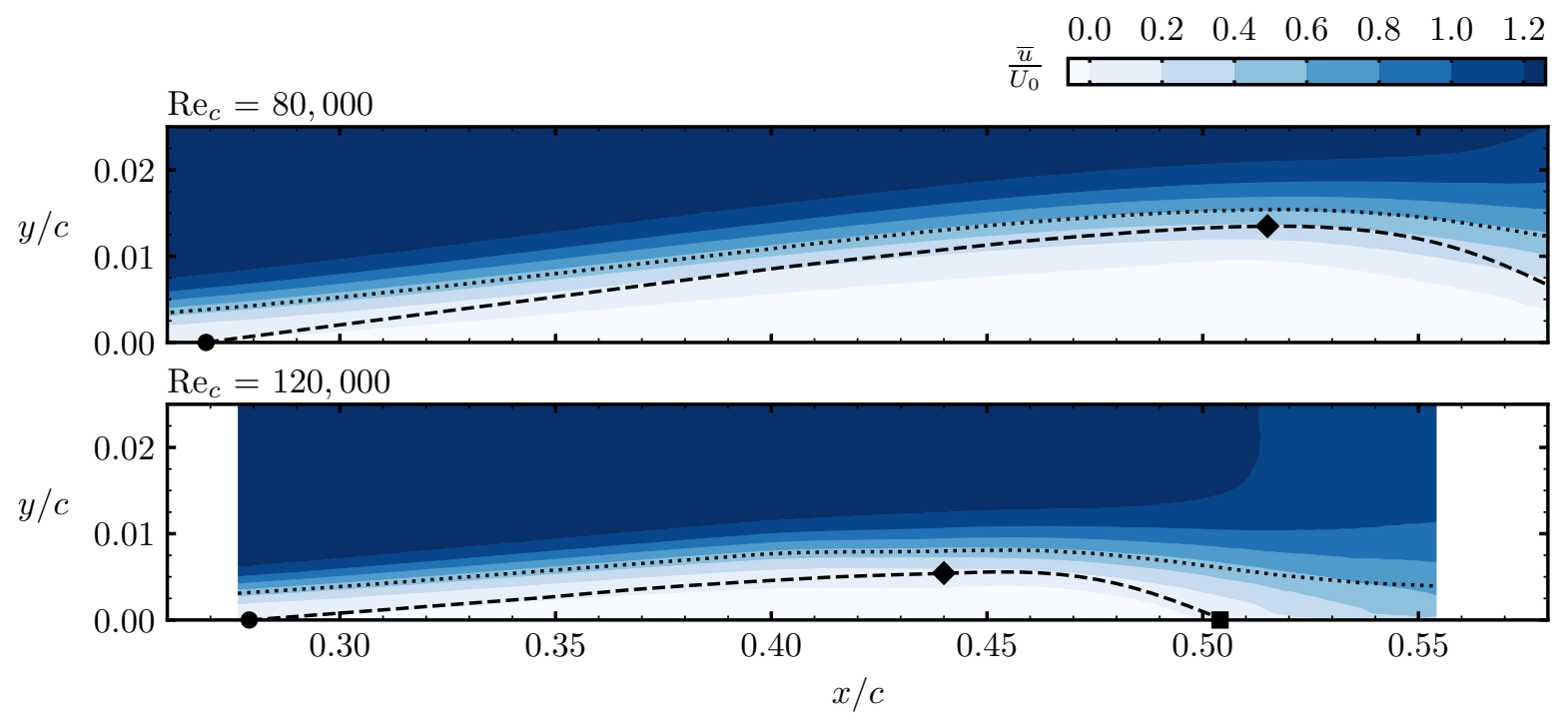

Figure 5.1: Contours of time-averaged streamwise velocity at each highlighted flow condition. The mean dividing streamline and displacement thickness are shown with dashed and dotted lines, respectively. Estimations of mean separation, transition, and reattachment are shown with circle, diamond, and square markers, respectively.

\subsection{PIV Measurements}

Two-component, planar PIV measurements were performed at the airfoil mid-span, as shown in Fig. 3.3b. Time-averaged streamwise velocity fields identify the presence of a laminar separation bubble at each Reynolds number (Fig. 5.1). The presented data were acquired at $100 \mathrm{~Hz}$, and processed using a sum-of-correlations scheme in the separation region, as shown in Fig. 3.4b. Lines indicating the mean dividing streamline and displacement thickness are shown with dashed and dotted lines, respectively. Similar to the results in Chapter 4, locations of mean separation and reattachment are estimated where a linear fit applied to the fore and aft portions of the mean dividing streamline intersects with the model surface. The location of mean transition is estimated at the point of maximum shape factor [56,75], as shown in Fig. 5.2. The estimated locations of mean separation, transition, and reattachment are indicated in Fig. 5.1 with circle, diamond, and square markers, respectively, and are summarized with respective uncertainty estimates in Table 5.1.

The point of mean separation is shown to shift downstream as the Reynolds number is increased. Although this result contradicts findings from previous studies on NACA 0018 airfoils at similar conditions employing PIV [11] and surface pressure measurements [7], the downstream shift is within the bounds of uncertainty such that the mean separation location can be considered constant. Mean transition and reattachment locations are shown to advance upstream as Reynolds number is increased (Table 5.1 and Fig. 5.1), 


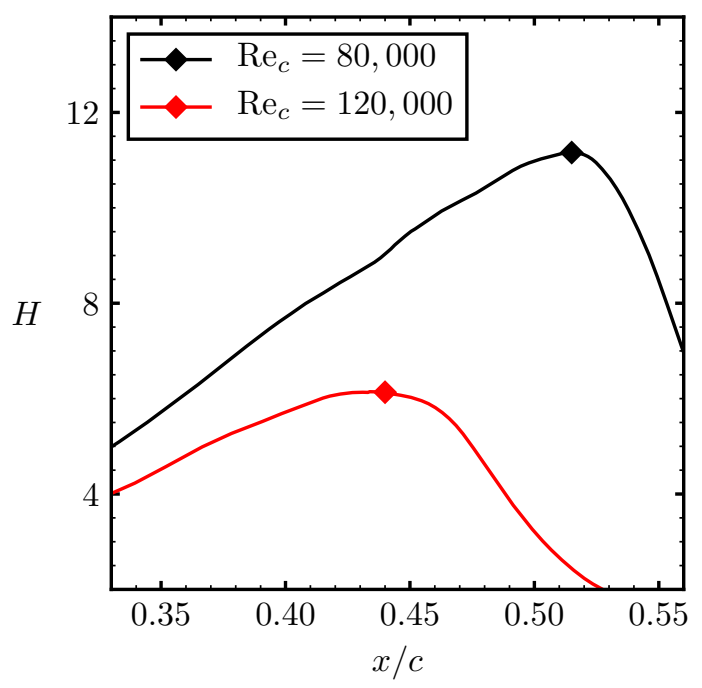

Figure 5.2: Shape factor distributions with associated transition estimates.

Table 5.1: Estimated characteristic locations based on PIV measurements.

\begin{tabular}{lccc}
\hline \hline & $x_{S} / c$ & $x_{T} / c$ & $x_{R} / c$ \\
\hline $\operatorname{Re}_{c}=80,000$ & $0.27 \pm 0.04$ & $0.52 \pm 0.01$ & $0.60 \pm 0.04$ \\
$\operatorname{Re}_{c}=120,000$ & $0.28 \pm 0.03$ & $0.44 \pm 0.02$ & $0.50 \pm 0.02$ \\
\hline \hline
\end{tabular}

agreeing with results from similar studies [7, 102]. This can be attributed to a larger wallnormal velocity gradient, resulting in a more pronounced instability within the separated shear layer. Therefore, higher Reynolds number flow experiences larger disturbance growth rates, consequently resulting in an earlier onset of mean transition and reattachment. Primarily due to advancement of the mean reattachment location, the spanwise extent of the laminar separation bubble is shown to decrease at higher Reynolds number, from $0.33 c$ at $\operatorname{Re}_{c}=80,000$ to $0.22 c$ at $\operatorname{Re}_{c}=120,000$.

Contours of fluctuating velocity components, shown in Figs. 5.3 and 5.4, offer insight into laminar separation bubble development and the associated transition process. The results exhibit distinct wall-normal peak values of fluctuating velocity, with single and multiple peaks observed in the wall-normal and streamwise components, respectively. The presence of such peaks agrees with observations from previous studies of laminar separation bubbles $[11,56,59]$. Other than the amplification of streamwise velocity fluctuations observed in the fore portion of the separation bubble, which has been attributed to shearlayer flapping [74], the most prominent amplification of velocity fluctuations is seen in the separated shear layer approaching the maximum bubble height location (Figs. 5.3 and 5.4). Elevated levels of fluctuating velocity upstream of mean transition can be attributed to 


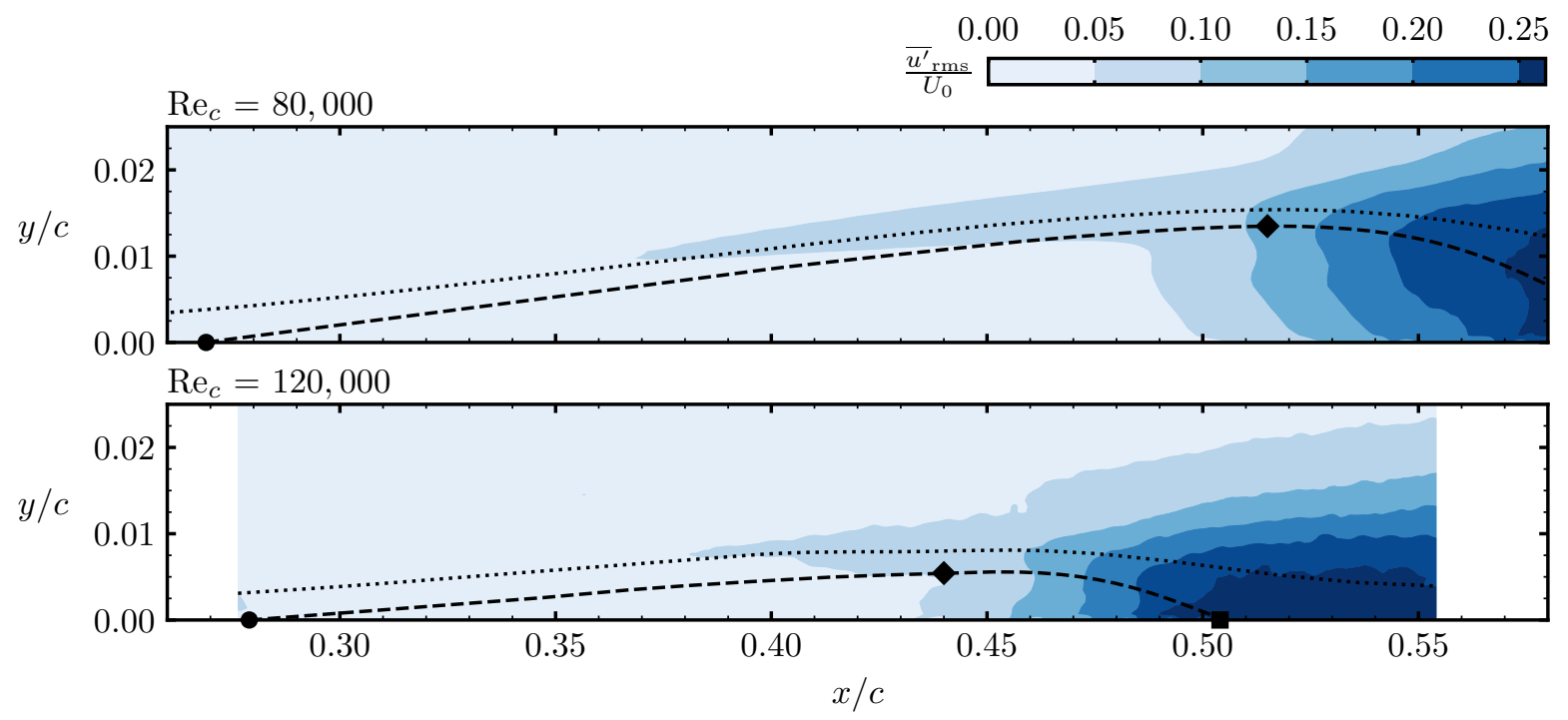

Figure 5.3: Contours of the streamwise RMS component of fluctuating velocity. The mean dividing streamline and displacement thickness are shown with dashed and dotted, respectively. Estimations of mean separation, transition, and reattachment are shown with circle, diamond, and square markers, respectively.

the formation and development of vortices in the separated shear layer. These vortices shed near the point of mean transition and tend toward the airfoil surface, where the associated enhanced momentum exchange in the near-wall region leads to reattachment in the mean sense [54]. This process is associated with significant amplification of Reynolds shear stress, as depicted in Fig. 5.5. Increased levels of RSS become evident near mean transition, increasing downstream and achieving maximum levels near mean reattachment.

The amplification of fluctuating velocity and Reynolds shear stress is associated with significant changes in separation bubble dynamics. The periodic process of vortex formation, shedding, and impingement for $\operatorname{Re}_{c}=120,000$ is depicted in Fig. 5.6 with contours of instantaneous spanwise vorticity. Individual vortices are tracked with dotted lines for illustration purposes. The onset of shear layer roll-up is shown to occur near $x=0.43 c$, just upstream of the mean transition location. This occurs once velocity fluctuations reach about $10 \%$ of the free stream velocity (Figs. 5.3 and 5.4), agreeing with observations from previous studies [54]. The shed vortices tend towards the surface, entraining fluid and momentum from the outer flow in the process. This amplifies the level of velocity fluctuations and Reynolds shear stress, which achieve maximum values in the vicinity of mean reattachment (Figs. 5.3-5.5), where vortices impinge on the surface and rapidly breakdown to smaller structures (Fig. 5.6). Mean reattachment is followed by a loss of coherence and diffusion of vorticity to the surrounding flow. As observed in experiments performed on the flat plate (Chapter 4), the mean flow reorientation present in the aft portion of the 


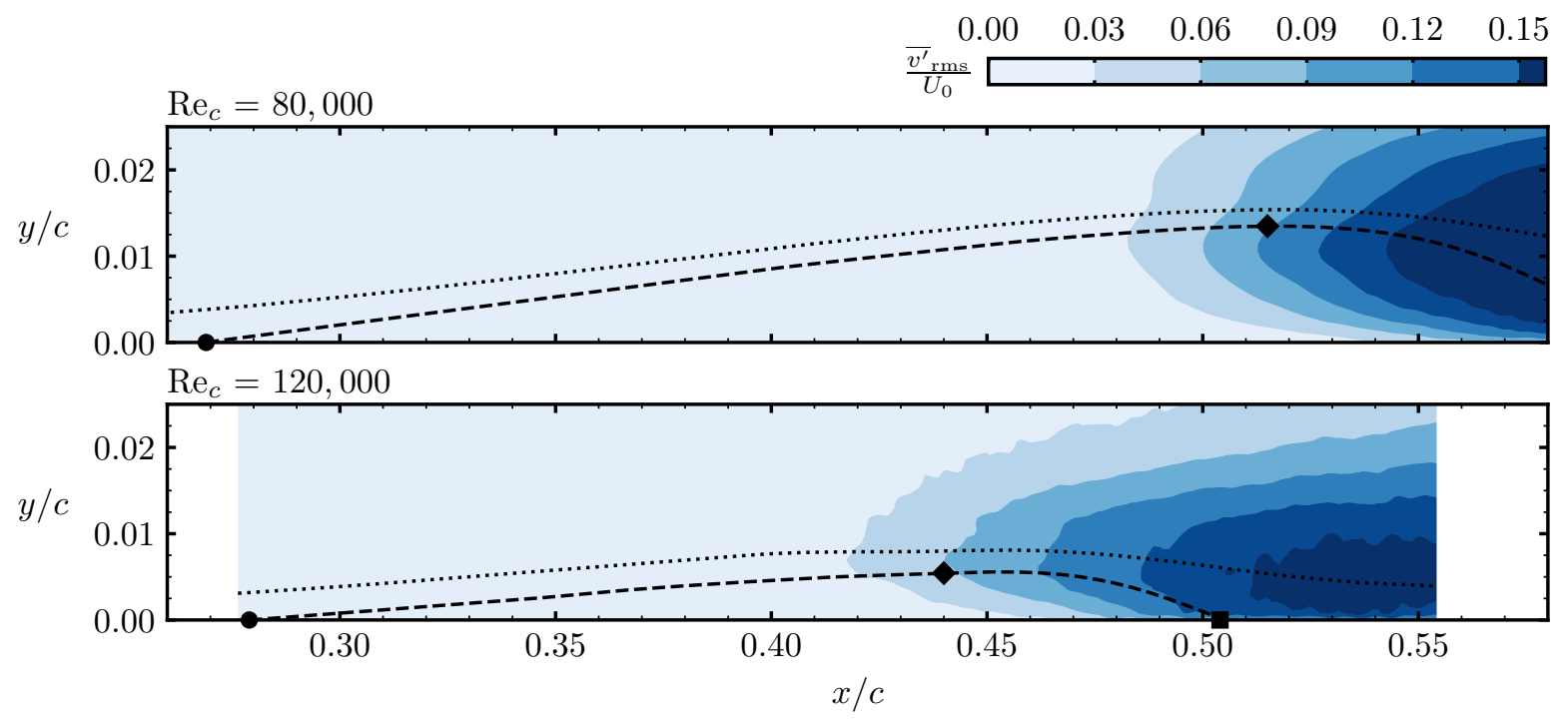

Figure 5.4: Contours of the wall-normal RMS component of fluctuating velocity. The mean dividing streamline and displacement thickness are shown with dashed and dotted, respectively. Estimations of mean separation, transition, and reattachment are shown with circle, diamond, and square markers, respectively.

separation bubble, coupled with a significant increase in near-wall velocity fluctuations, is expected to result in a notable increase of convective heat transfer intensity.

\subsection{Time-Averaged Thermal Analysis}

Measurements of temperature on the airfoil surface were realized with the use of infrared thermography, following the configuration shown in Fig. 3.5b. Distributions of surface temperature are analyzed in this section and related to the flow development presented in Section 5.1. Methodologies for characterizing laminar separation bubbles based on timeaveraged surface temperature distributions, as proposed in Section 4.2, are evaluated on the airfoil surface. Locations of mean separation, transition, and reattachment are estimated, and the proposed methodologies are compared in terms of identification robustness and accuracy.

Time-averaged surface temperature distributions are presented in Fig. 5.7. Locations of mean separation, transition, and reattachment from PIV measurements are shown with dotted, dashed, and dash-dotted lines, respectively. A spatial filter with a kernel of 5px was applied to the presented temperature distributions to mitigate spurious data. A band of elevated surface temperature is observed for each flow condition, roughly coinciding with the marked location of the laminar separation bubble estimated from PIV measure- 


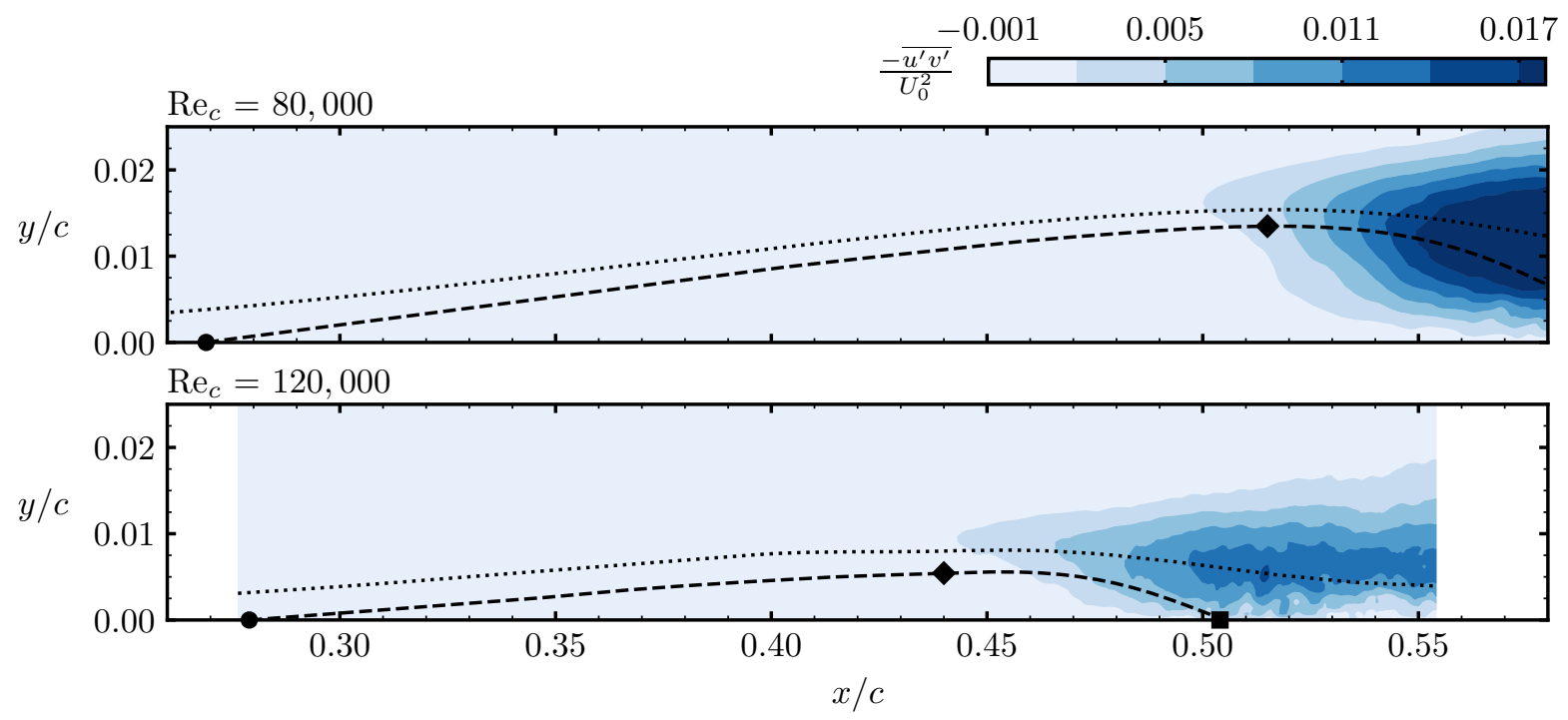

Figure 5.5: Reynolds shear stress contours. The mean dividing streamline and displacement thickness are shown with dashed and dotted lines, respectively. Estimations of mean separation, transition, and reattachment are shown with circle, diamond, and square markers, respectively.

ments. As the Reynolds number is increased, the downstream edge of the increased surface temperature band shifts in the upstream direction, reducing the bands streamwise extent. These results support observations from the flat plate experiments (Fig. 4.10), and claims of Montelpare \& Ricci [27] suggesting a band of increased surface temperature can be used as an indicator of laminar separation bubble presence.

Similar to thermal behaviour observed on the flat plate (Fig. 4.10), the airfoil experiences a gradually increasing surface temperature upstream of mean separation due to near-wall deceleration in the attached laminar boundary layer flow (Eq. 2.7). The nearstagnant flow in the vicinity of mean separation is associated with minimal convective heat transfer intensity [34,114], resulting in a maximum temperature attained in the fore portion of the separation bubble. Intensified cooling of the surface is initiated with the rapid onset of transition, where increased levels of fluid entrainment and near-wall velocity fluctuations progressively enhance the magnitude of convective heat transfer in the aft portion of the bubble. The surface temperature reaches a minimum level near mean reattachment, where mean oblique flow impingement combined with high near-wall velocity fluctuations induced by shear layer vortices (Figs. 5.3, 5.4, and 5.6) produce maximal convective heat transfer at the surface. As the turbulent boundary layer redevelops downstream, a gradual streamwise decrease in the convective heat transfer coefficient occurs, with the rate of heat transfer decay being characteristically lower than in the laminar boundary layer upstream of separation [114]. This brings about a mild increase in surface temperature towards the 


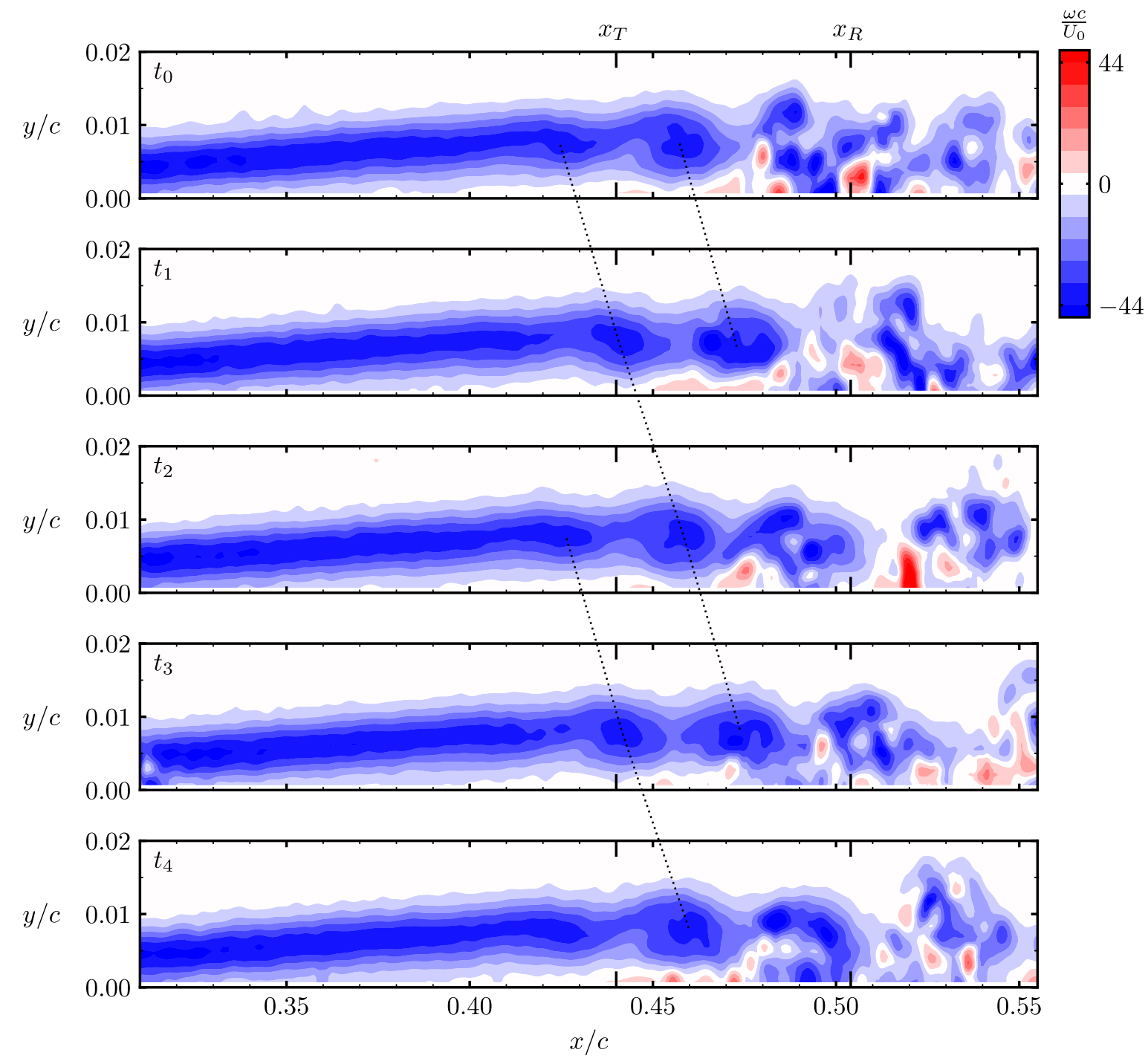

Figure 5.6: Contours of instantaneous spanwise vorticity at $\operatorname{Re}_{c}=120,000$. Each frame is separated by $667 \mu \mathrm{s}$, and the locations of mean transition and reattachment are indicated with extended tick markers. 


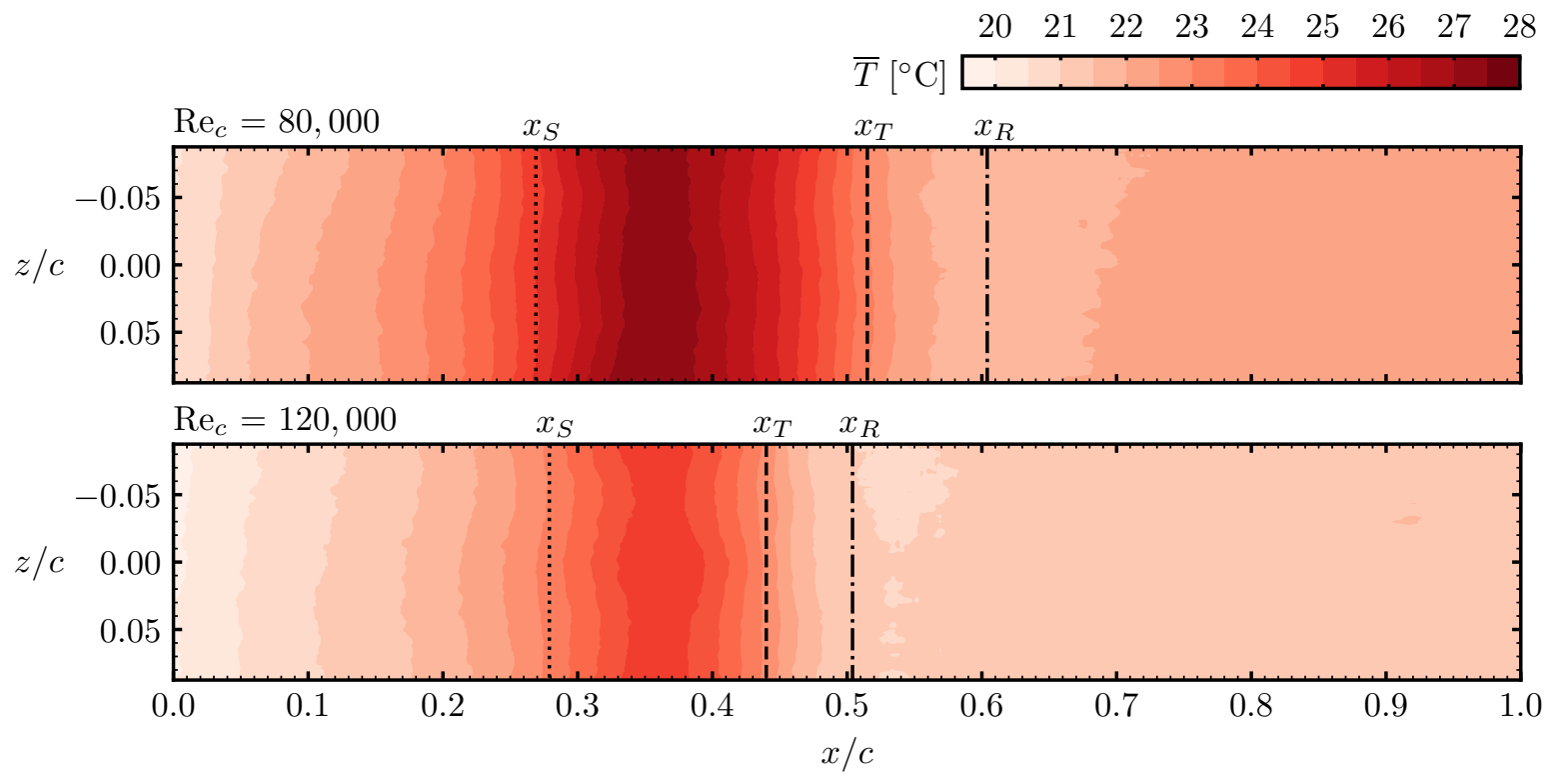

Figure 5.7: Contours of time-averaged temperature measured on the airfoil surface. Estimated locations of mean separation, transition, and reattachment from PIV measurements are shown with dotted, dashed, and dash-dotted lines, respectively.

trailing edge of the airfoil Fig. 5.7.

For both Reynolds numbers investigated, distributions of surface temperature exhibit adequate spanwise uniformity within the infrared field of view (Fig. 5.7). Therefore, spanwise-averaged temperature distributions can be used for characterization of the separated region, as discussed in Section 4.2. Distributions of spanwise-averaged $\left(\bar{T}-T_{\infty}\right)$, and corresponding streamwise gradients, are shown for each Reynolds number in Fig. 5.8. An additional 5px spatial filter is applied to all spanwise-averaged data for visual clarity. The points of mean separation and transition are estimated at locations of maximum and minimum streamwise temperature gradient, respectively. The mean reattachment location is estimated where the surface temperature attains a minimum value, downstream of the absolute maximum. Mean separation, transition, and reattachment locations are indicated in Fig. 5.8 with circle, diamond, and square markers, respectively. These locations are summarized in Table 5.2 along with their associated uncertainty estimates.

Identified locations of mean separation, transition, and reattachment, following the surface temperature-based methodology described above, yield good agreement with the corresponding estimates obtained from PIV measurements (Table 5.2). The estimated location of mean separation remains invariant with the increase in Reynolds number, similar to the trend observed in PIV measurements within the experimental uncertainty. The estimated locations of mean transition and reattachment from surface temperature mea- 


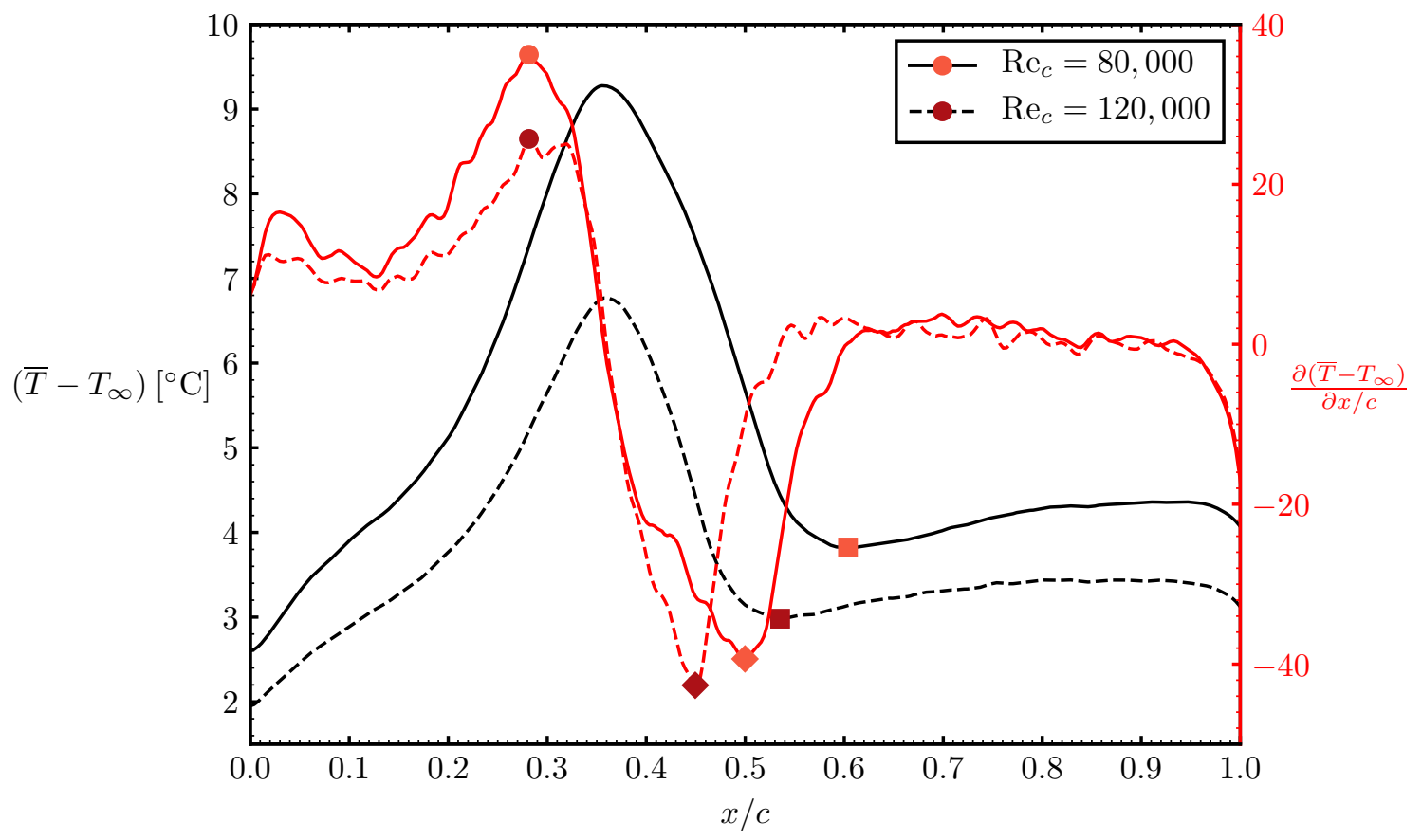

Figure 5.8: Time- and spanwise-averaged distributions of surface temperature and its streamwise gradient. Estimated locations of mean separation, transition and reattachment are indicated with circle, diamond, and square markers, respectively.

surements are shown to shift in the upstream direction with increasing Reynolds number, reducing the overall extent of the separated region, agreeing with trends observed in PIV measurements (Fig. 5.1) and previous studies [7,10,102]. Relative to observations from flat plate experiments (Fig. 4.11), the surface temperature distributions in Fig. 5.8 facilitate a more precise identification of mean separation and reattachment at the locations of maximum temperature gradient, and minimum surface temperature, respectively. This is likely attributed to the presence of an air-filled core in the NACA 0018 airfoil design, as extra consideration was paid to minimizing the wall-normal conductive heat flux compared to flat plate experiments. The addition of an air-filled core acts as an insulating layer, ensuring the surface heat transfer is dominated by forced convection, as shown with a full energy balance in Appendix C.

The presented characterization of surface temperature distributions requires assumptions regarding radiative and conductive heat fluxes to be made (Eq. 2.1). As explained in Section 4.2, uniformity of radiative heat flux is difficult to achieve in practice without the use of specialized surface heating elements. To evaluate the validity of these assumptions, the distribution of mean surface temperature in quiescent conditions is shown in Fig. 5.9. Similar to flat plate experiments (Fig. 4.12), a non-uniform streamwise distribution of 


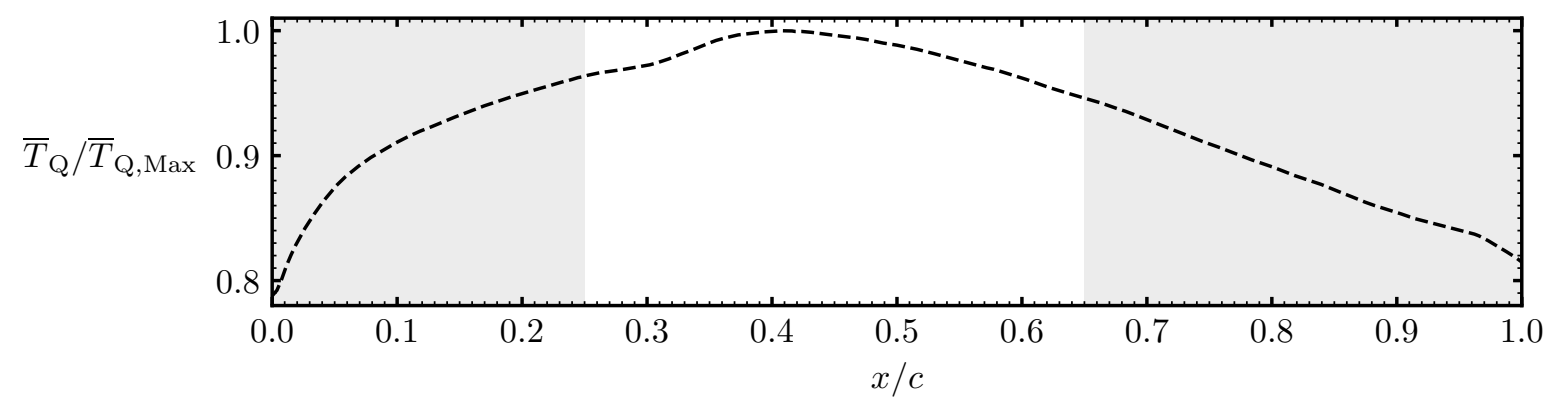

Figure 5.9: Time- and spanwise-averaged distribution of normalized surface temperature in quiescent conditions. The unshaded region locates approximate bounds where laminar separation bubbles were observed in this study.

surface temperature is observed in quiescent conditions. Variations in quiescent surface temperature can be attributed to non-uniformities in radiative heat flux, differences in perceived emissivity resulting from airfoil curvature, and non-zero streamwise conductive heat flux. These non-uniformities are shown to be less than approximately $5 \%$ within the area associated with the laminar separation bubble (Fig. 5.9). Computing distributions of $h^{*}$ (Eq. 4.3) along the chord, similar to the procedure followed in Section 4.2, allows for partial correction of the present non-uniformities. The variation of $h^{*}$ along the airfoil surface is shown in Fig. 5.10, along with distributions of corresponding streamwise gradients. Distributions of $h^{*}$ exhibit overall trends expected based on the earlier comparative analysis of flow field measurements (Figs. 5.1 and 5.3-5.5) and surface temperature distributions (Figs. 5.7 and 5.8). In particular, the convective heat transfer coefficient decreases towards mean separation, attaining minimum values within the fore portion of the separation bubble. As the separated shear layer undergoes transition and reattaches to the surface, the convective heat transfer coefficient rapidly increases, attaining maximum values in reattaching region (Fig. 5.10). Downstream of this location, a gradual decrease in the convective heat transfer coefficient is observed as the attached turbulent boundary layer develops.

Locations of mean separation, transition, and reattachment can be estimated from distributions of $h^{*}$, shown in Fig. 5.10 with circle, diamond, and square markers, respectively. Similar to the methodology implement for flat plate flow (Section 4.2), the locations of mean separation and transition are estimated where the streamwise gradient of $h^{*}$ achieves a minimum and maximum value, respectively. The absolute maximum value of $h^{*}$ in the aft portion of the separation bubble is used to estimate the location of mean reattachment. Characteristic points identified with this methodology are summarized with corresponding uncertainty estimates in Table 5.2, where they are compared to estimations from PIV measurements. The location of mean separation is shown to shift downstream as Reynolds 


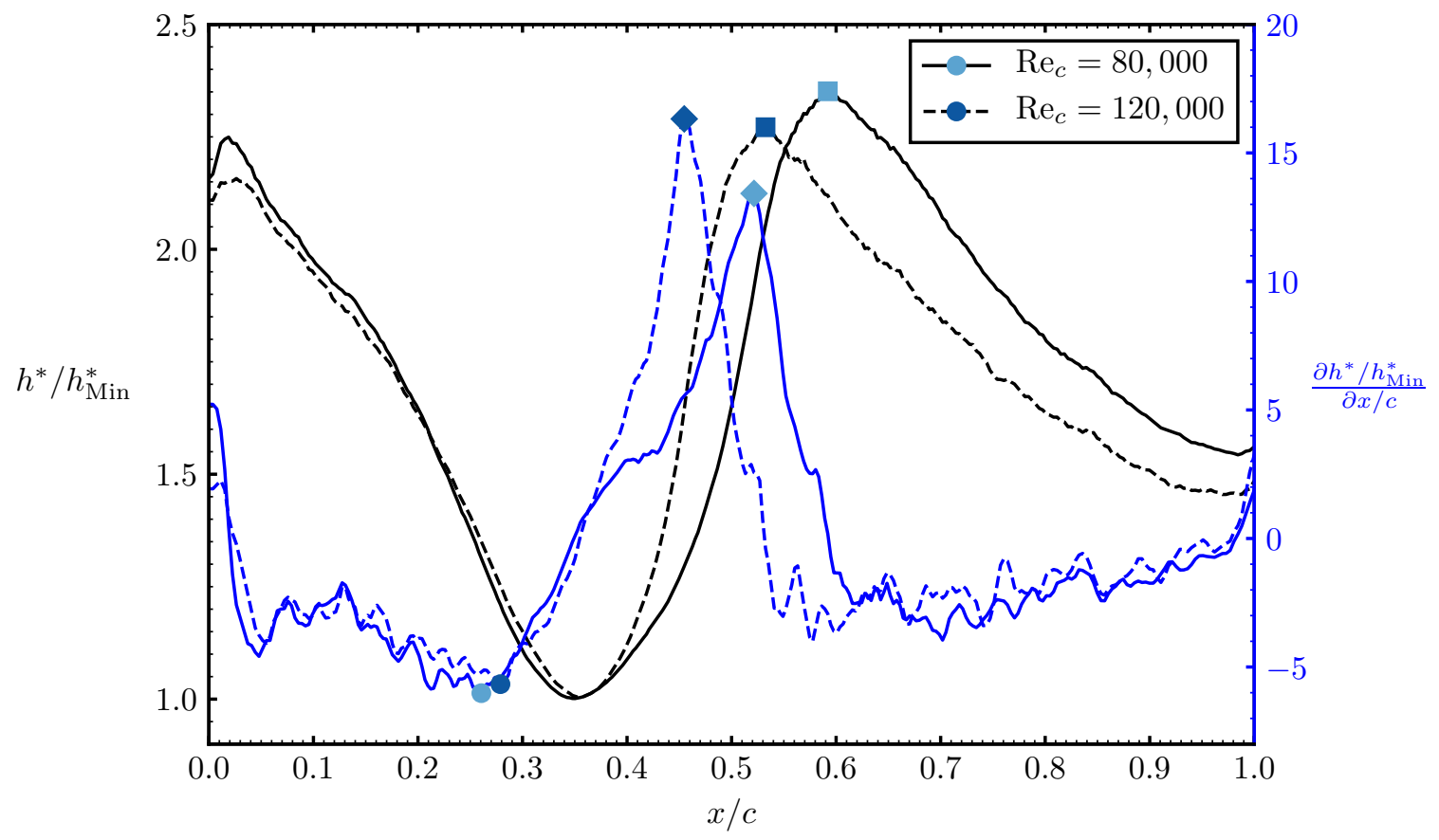

Figure 5.10: Time- and spanwise-averaged distributions of $h^{*}$ and its streamwise gradient. Estimated locations of mean separation, transition and reattachment are indicated with circle, diamond, and square markers, respectively.

number is increased, similar to the downstream progression observed in PIV measurements. The locations of mean transition and reattachment are shown to advance in the upstream direction as Reynolds number is increased, agreeing within uncertainty bounds of the PIV estimations.

Comparing the results presented in Figs. 4.13 and 5.10, it can be seen how minimizing the downward heat flux can improve the precision of estimated characteristic locations based on distributions of the convective heat transfer coefficient. Compared to the distributions of $h^{*}$ presented for the flat plate experiments (Fig. 4.13), a more distinct location of minimum streamwise gradient is observed, allowing for a more robust estimation of mean separation. Furthermore, distributions of $h^{*}$ on the airfoil surface exhibit a more pronounced peak $h^{*}$ location used to identify mean reattachment, resulting in an $h^{*}$ distribution which more closely resembles expected trends from DNS investigations [114].

As shown in Table 5.2, accounting for a relatively minor non-uniformity in quiescent surface temperature and computing distributions of convective heat transfer coefficient did not have an appreciable effect on the characterization of the laminar separation bubble. However, distributions of $h^{*}$ display an improved agreement with the expected variation of convective heat transfer coefficient beneath a laminar separation bubble put forth by 
Table 5.2: Estimated locations of mean separation, transition, and reattachment from PIV and temperature-based measurements on the airfoil.

\begin{tabular}{ccccc}
\hline \hline Flow Condition & Measurement Technique & $x_{S} / c$ & $x_{T} / c$ & $x_{R} / c$ \\
\hline \multirow{3}{*}{$\operatorname{Re}_{c}=80,000$} & PIV & $0.27 \pm 0.04$ & $0.52 \pm 0.01$ & $0.60 \pm 0.04$ \\
& $T$ Distribution & $0.28 \pm 0.03$ & $0.50 \pm 0.03$ & $0.60 \pm 0.02$ \\
& $h^{*}$ Distribution & $0.26_{-0.06}^{+0.03}$ & $0.52 \pm 0.02$ & $0.59 \pm 0.02$ \\
& $h$ Distribution & $0.31_{-0.04}^{+0.11}$ & $0.49_{-0.09}^{+0.10}$ & $0.58_{-0.05}^{+0.08}$ \\
& PIV & $0.28 \pm 0.03$ & $0.44 \pm 0.02$ & $0.50 \pm 0.02$ \\
$\operatorname{Re}_{c}=120,000$ & $T$ Distribution & $0.28_{-0.02}^{+0.05}$ & $0.45 \pm 0.02$ & $0.54 \pm 0.02$ \\
& $h^{*}$ Distribution & $0.28_{-0.08}^{+0.04}$ & $0.45 \pm 0.02$ & $0.53 \pm 0.02$ \\
& $h$ Distribution & $0.32_{-0.08}^{+0.05}$ & $0.46_{-0.09}^{+0.08}$ & $0.54_{-0.06}^{+0.10}$ \\
\hline \hline
\end{tabular}

Spalart \& Strelets [114], as secondary heat transfer modes are partially compensated for. Therefore, it is expected that distributions of $h^{*}$ more accurately resemble the actual variation of convective heat transfer intensity within a laminar separation bubble.

\subsection{Transient Heat Transfer Analysis}

Results presented in Section 5.2 demonstrate how distributions of convective heat transfer coefficient can be estimated by relating steady-state surface temperature measurements in quiescent and flow conditions, where constant radiative heat flux is assumed between such conditions. However, it necessitates time-averaged measurements of surface temperature in quiescent conditions, which may not be possible in some situations, and otherwise requires a relatively uniform or known heating/cooling of the model surface. Thus, it is of interest to explore a method that would allow for estimation of the convective heat transfer coefficient distribution directly from in-flow measurements, with less restrictive requirements for incident heating/cooling. This can be achieved using transient surface temperature measurements. Specifically, a transient decline of surface temperature can be measured with infrared thermography, initiating once the incident radiative heating is stopped. The measured transient surface temperature history can then be related to the convective heat transfer coefficient through the first law of thermodynamics. In the formulation presented below, conduction and radiative cooling are neglected, and a uniform temperature distribution is assumed within a small volume element, $V$, of the airfoil model shell, with the surface temperature measured on a small area element, $A$. The rate of change of internal energy of the shell element can then be related to convective heat transfer as:

$$
\rho_{s} V c_{p, s} \frac{d T}{d t}=-h A\left(T-T_{\infty}\right)
$$


Equation 5.1 can be written as:

$$
\frac{d T}{d t}=-K h\left(T-T_{\infty}\right)
$$

where $K=A / \rho_{s} c_{p, s} V$ is a dimensional constant. Integrating Eq. 5.2 yields:

$$
T(t)=\left(T_{0}-T_{\infty}\right) \exp (-K h t)+T_{\infty}
$$

where $T_{0}$ is the temperature of a given surface element at the transient onset, i.e. $T(t=0)$. Thus, the local convective heat transfer coefficient can be estimated based on the rate of decay of measured surface temperature. To facilitate this, infrared measurements were initiated at time $t=t_{0}=0$, while radiative heating was simultaneously removed by turning off the halogen lamps and covering the test section roof with a neoprene sheet to minimize residual radiative heat flux. Infrared measurements were acquired at a frequency of $1 \mathrm{~Hz}$ for a total of 30 min per Reynolds number. The transient surface decline was then fit with an exponentially decaying function at each location on the airfoil (Eq. 5.3), from which, the product $K h(x, z)$ was extracted. The obtained $K h(x, z)$ estimates were then averaged across the span $(z)$, yielding a spanwise-average distribution of $K h(x)$. A spatial filter with a kernel of 5px was applied to the results presented in Fig. 5.12, where a 10px kernel was applied to the results shown in Fig. 5.13.

An example of the measured transient surface temperature decline is shown on a semilogarithmic scale in Fig. 5.11. The data presented in Fig. 5.11 serve to illustrate the effect of transient duration on the exponential fit, which is related to the estimate of $K h$. The exponential fit is shown to reasonably model the measured surface temperature during the initial transient, before deviation from the exponential trend is observed. The degradation of model accuracy at higher times is likely due to the progressively increasing influence of conduction on internal energy redistribution within the model, which is neglected in the present formulation (Eq. 5.1). The effect of transient sample duration on the estimated $K h$ distribution is illustrated in Fig. 5.12. It is shown that the number of data points considered in the exponential fit should be maximized in order to reduce spatial fluctuations. However, only the data which follow the exponential trend should be considered, as including subsequent data points deteriorates the accuracy of the convective heat transfer coefficient estimate. Considering the results presented in Figs. 5.11 and 5.12, a transient duration of $6 \mathrm{~s}$ was selected for the subsequent analysis as a compromise between random error reduction and model accuracy.

Distributions of the convective heat transfer coefficient, and its streamwise gradient, are shown in Fig. 5.13. Presented distributions are normalized by the minimum value of $K h$ along the chord, yielding distributions of $h / h_{\mathrm{Min}}$. Agreeing with the estimated varia- 


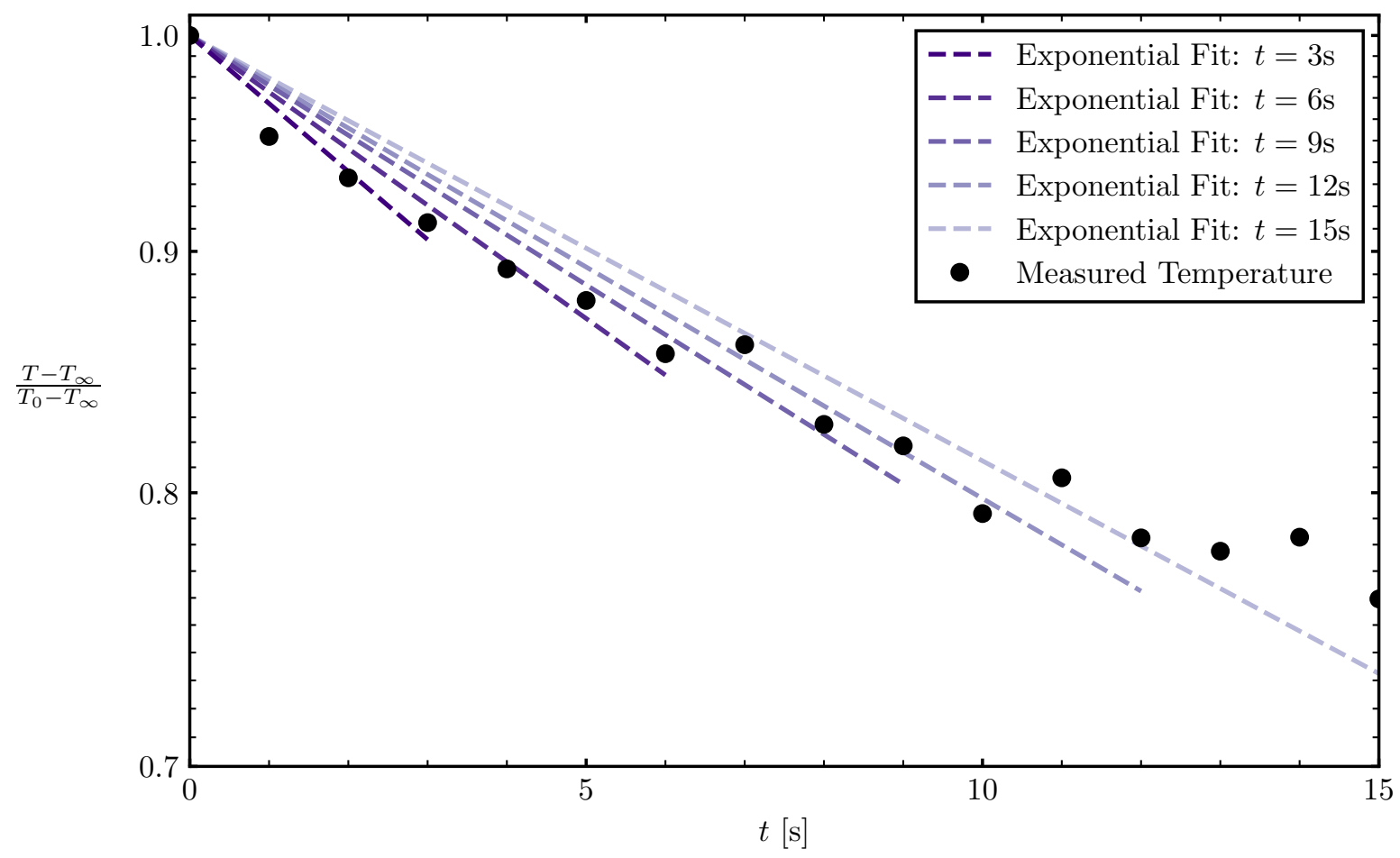

Figure 5.11: Transient development of surface temperature resulting from a step change in radiative heating at $x \approx 0.5 c$ and $\mathrm{Re}_{c}=120,000$. Dashed lines indicate exponential fits (Eq. 5.3) applied with different transient durations. The $y$-axis is plotted on a natural logarithmic scale.

tion of convective heat transfer coefficient from quasi-steady state measurements shown in Fig. 5.10, the profiles in Fig. 5.13 exhibit trends expected due to the laminar separation bubble presence. More specifically, a reduction in convective heat transfer is observed upstream of separation due to laminar boundary layer development and deceleration of the near-wall flow. Minimum convective heat transfer is attained within the fore portion of the separated region, followed by rapid intensification of convective heat transfer associated with the onset of transition and subsequent reattachment.

Being based on a single experimental realization, the estimated distributions of convective heat transfer coefficient exhibit significant spatial fluctuations, which are amplified substantially in estimates of the streamwise gradient (Fig. 5.13). Although a location of minimum streamwise gradient immediately upstream of the minimum $h$ location can be identified and used to locate separation, the magnitude of the corresponding extremum is comparable to the extrema at surrounding locations. Therefore resulting in an identification of separation associated with relatively high levels of methodological uncertainty, as shown in Fig. D.7. A less precise, yet less ambiguous, characterization of the separation 

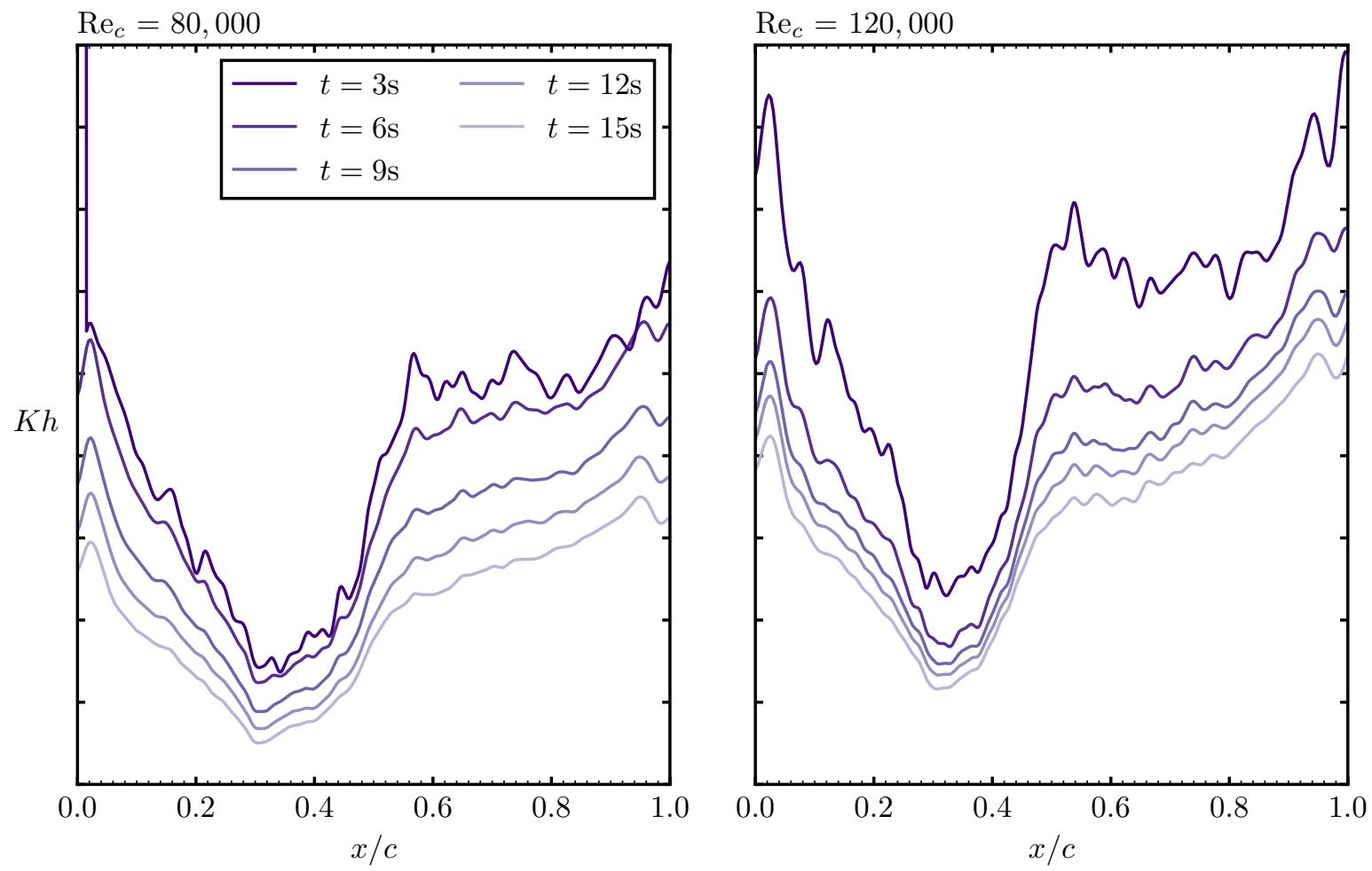

Figure 5.12: Distribution of convective heat transfer coefficient calculated from transient thermal decline plotted on an arbitrary scale. Numerous profiles correspond to different transient durations considered for fitting the thermal time series.

location is implemented here, where separation is estimated at the location of minimum convective heat transfer coefficient. Although this location is likely downstream of the true separation location, it is associated with significantly less methodological uncertainty as it is not based on a gradient distribution. The location of transition is estimated at the location of maximum gradient in the convective heat transfer coefficient, which can be located within reasonable uncertainty bounds due to the relatively pronounced peak value.

In addition to significant noise propagation, a clear maximum convective heat transfer coefficient is not attained near reattachment, as the value of $h / h_{\text {Min }}$ continues to increase towards the trailing edge. This can be attributed to insufficient thermal and temporal measurement resolution, and the deterioration of assumptions made in the initial energy balance (Eqs. (5.1)-(5.3)). The weakly defined maximum convective heat transfer coefficient suggest that the increased convective heat flux associated with vortex impingement was not sufficiently resolved within the measurement resolution, as comparable levels of estimated convective heat transfer coefficient are observed in the developing turbulent boundary layer downstream. The convective heat transfer coefficient is shown to significantly increase towards the trailing edge, which is likely due to the implied assumption 


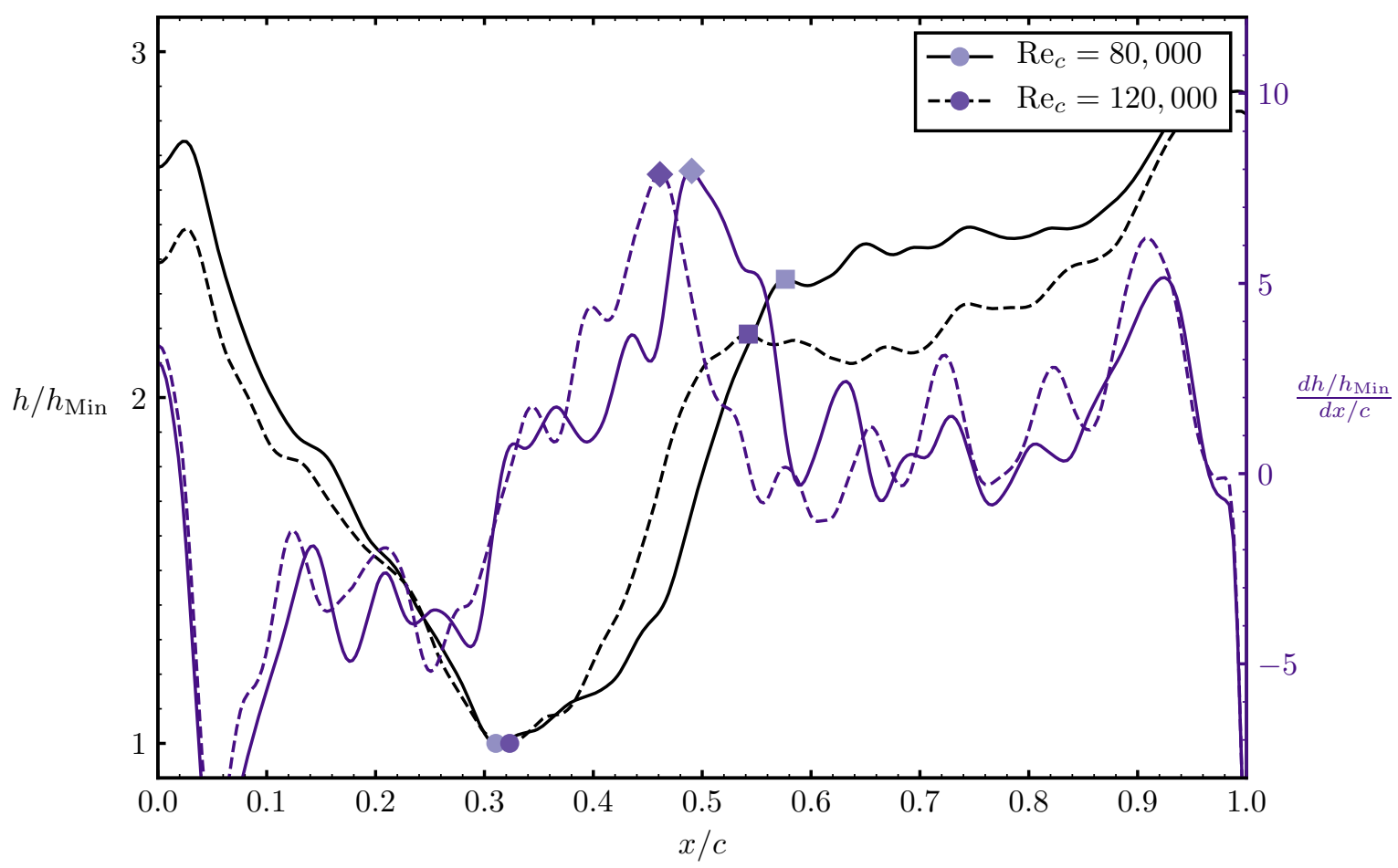

Figure 5.13: Spanwise-averaged distribution of normalized convective heat transfer coefficient and its streamwise gradient calculated with a transient time of $6 \mathrm{~s}$. Estimated locations of separation, transition and reattachment are indicated with circle, diamond, and square markers, respectively.

of a spatially invariant value of $K$. More realistically, as the airfoil thickness decreases below twice the shell thickness, the quotient $A / V$ in $K$, an inverse of wall-thickness, increases due to the thinning airfoil geometry. This results in an increase of $K$ downstream of $x \approx 0.8 c$, artificially increasing the convective heat transfer coefficient. Considering the decreased model accuracy downstream of transition, the reattachment location is identified at the first local maximum of convective heat transfer coefficient downstream of the rapid increase of $h$, which coincides with the maximum $h$ location attained at shorter transient durations (Fig. 5.12). The locations of separation, transition, and reattachment are shown in Fig. 5.13 with circle, diamond, and square markers, respectively, while a summary of these locations and their associated uncertainty is provided in Table 5.2.

The locations of separation, transition, and reattachment estimated from distributions of $h / h_{\text {Min }}$ (Fig. 5.13) demonstrate reasonable agreement with locations established from PIV measurements (Table 5.2). Additionally, the values of $h / h_{\text {Min }}$ observed in Fig. 5.13 are of similar magnitude to those of $h^{*} / h_{\text {Min }}^{*}$ presented in Fig. 5.10. Although a reasonable characterization of the separation bubble was obtained, distributions of the convective 
heat transfer coefficient streamwise gradient exhibit significant spatial fluctuations due to increased noise propagation, requiring averaged results from several transient decay cycles in order to reduce the fluctuations to levels observed in time-averaged methodologies. Additionally, a weakly defined local maximum convective heat transfer coefficient is observed in the vicinity of reattachment, followed by an increase towards the trailing edge, differing from expected trends (Fig. 5.10) and previous investigations [114]. The aforementioned effects inherently reduce the precision associated with the estimated characteristic locations and introduce increased levels of methodological uncertainty relative to the estimations from time-averaged methodologies.

A comparison of the results from time-averaged thermal measurements (Section 5.2) with those established from transient thermal measurements (Section 5.3) is shown in Table 5.2. Although differences between the presented methodologies exist at specific characteristic points, the locations identified with each methodology are shown to agree with PIV estimations within uncertainty bounds. However, differences between time-averaged and transient-based methodologies are observed in the magnitude of methodological uncertainty associated with the estimated characteristic points. Thermal fluctuations, which are reduced through temporal averaging, are inherently more pronounced in the transient-based results. Such fluctuations are amplified in the streamwise gradient distribution, affecting the precision of separation and transition estimates, to the extent where separation is more precisely identified at the minimum convective heat transfer location. However, a similar reduction in measurement noise can be achieved with the transient-based methodology by repeating the measurement cycle multiple times and averaging the results. As this is generally more time consuming, the transient methodology should be restricted to cases where quasi-steady measurements are not available, or the underlying assumptions employed in this analysis cannot be sufficiently satisfied. 


\section{Chapter 6}

\section{Conclusions}

The feasibility of characterizing laminar separation bubbles using infrared thermography was evaluated in a comparative analysis on two model geometries. Results are first presented from a study of laminar separation bubbles formed on a flat plate, upstream of a vertically mounted fence. Three highlighted flow conditions are analysed in detail, pertaining to fence height-based Reynolds numbers of $\operatorname{Re}_{L}=27,000,36,000$, and 45, 000 . Secondly, results from a follow-up study of laminar separation bubbles formed on a NACA 0018 airfoil are presented. Here, two main flow conditions are highlighted, pertaining to an angle of attack of $4^{\circ}$ and chord-based Reynolds numbers of $\operatorname{Re}_{c}=80,000$ and 120, 000 . Measurements of PIV, and surface pressure for the flat plate study, are used to provide a topological description of the laminar separation bubble formed at each flow condition. Infrared thermography is utilized to obtain surface temperature distributions, which are related to PIV observations and used to estimate the location and extent of the separated region.

\subsection{Laminar Separation Bubble Development On a Flat Plate Geometry}

It is demonstrated that typical laminar separation bubbles can be attained upstream of a vertically mounted fence on a flat plate. Pressure and velocity field measurements show that as the Reynolds number is increased, the mean separation location moves downstream, while transition and reattachment locations advance in the upstream direction. This reduces streamwise extent of the separation bubble at higher Reynolds number, agreeing with previous experimental investigations [7, 10, 102].

Statistics of fluctuating velocity and Reynolds shear stress provided insight into the 
transition process, and allowed for expected trends in convective heat transfer to be established. Relatively low amplitudes of fluctuating velocity and Reynolds shear stress are observed in the fore portion of the separated region, with significant increases occurring with the rapid onset of transition in the separated shear layer. The magnitudes of fluctuating velocity and Reynolds shear stress are amplified throughout the transition region, achieving maximum levels near mean reattachment. Periodic shear layer roll-up and shedding is observed, which is linked to the significant increase in fluctuating velocity and Reynolds shear stress throughout the aft portion of the separation bubble.

Measurements of time-averaged surface temperature portray trends expected from PIV observations regarding the variation of convective heat transfer coefficient within the laminar separation bubble. A band of increased temperature exists on the model surface where a laminar separation bubble is detected with PIV measurements. A quantitative laminar separation bubble characterization technique has been proposed based on PIV observations and results from previous investigations. The locations of mean separation and transition have been identified where spanwise-averaged distributions of the streamwise $\left(\bar{T}-T_{\infty}\right)$ gradient achieved maximum and minimum values, respectively. The location of mean reattachment has been identified at a minimum value of $\left(\bar{T}-T_{\infty}\right)$, approximately corresponding to a maximum of the convective heat transfer coefficient. Mean separation and transition locations determined from the proposed methodology have been shown to exhibit good agreement with locations estimated from PIV and surface pressure measurements. However, this methodology results in mean reattachment estimations which remain relatively unchanged with Reynolds number, which is attributed to the non-uniformity of conductive and radiative heat fluxes not accounted for in this simplified approach.

A differential energy balance has been employed to mitigate effects of non-uniform conductive and radiative heat flux, yielding an estimated streamwise distribution of normalized convective heat transfer coefficient, $h^{*}$. Following a similar methodology as that developed from the characterization of surface temperature distributions, locations of mean separation, transition, and reattachment have been identified and subsequently compared to those estimated from surface pressure and PIV measurements. The results demonstrate the importance of considering non-uniformities in conductive and radiative heat fluxes. Although similar quantitative estimates of the key locations along the laminar separation bubble have been achieved between both methodologies, a more accurate variation of convective heat transfer beneath the laminar separation bubble is likely to be achieved with distributions of $h^{*}$. This is due to the less restrictive assumptions regarding incident radiative heat flux in distributions of $h^{*}$ compared to those of mean surface temperature. 


\subsection{Laminar Separation Bubble Development On a NACA 0018 Airfoil}

Time-averaged fields of streamwise velocity from PIV measurements demonstrate the presence of a laminar separation bubble at each investigated Reynolds number, allowing for estimation of the mean separation, transition, and reattachment locations. With increasing the Reynolds number, the mean separation location remains relatively unchanged, while upstream advancement of mean transition and reattachment takes place, agreeing with previous experimental investigations of laminar separation bubbles formed on similar airfoil geometries [7, 8, 102]. Contours of fluctuating velocity, Reynolds shear stress, and instantaneous spanwise vorticity have been considered, providing insight into the transition process and expected thermal development due to laminar separation bubble presence.

Infrared measurements of time-averaged surface temperature demonstrated thermal behaviour which agrees with expected trends established from PIV observations. The locations of mean separation, transition, and reattachment estimated from temperature distributions following the previously established methodology have been shown to agree with those determined from PIV measurements within the bounds of uncertainty. In order to account for non-uniformities in radiative heat flux, streamwise conduction, and surface emissivity, time-averaged surface temperature measurements have been used to estimate the distribution of normalized convective heat transfer coefficient, $h^{*}$. These distributions provide estimates for mean separation, transition, and reattachment locations that agree with those estimated from PIV measurements. The characteristic locations estimated with both time-averaged methodologies were shown to be nearly identical at each flow condition; however, increased accuracy is likely to be achieved with distributions of $h^{*}$ as non-uniformities in radiative heat flux can be partially accounted for.

A differential energy balance has been formulated in order to directly calculate the variation of convective heat transfer coefficient from the transient decay of surface temperature. The calculated variation of convective heat transfer coefficient has been shown to reasonably follow expected convective heat transfer trends up to the reattachment location. Additionally, these distributions have been shown to estimate locations of mean separation, transition, and reattachment which agree within uncertainty bounds of PIV estimations. However, the variation of convective heat transfer coefficient is sensitive to the duration over which measurements were performed, and is inherently sensitive to random measurement errors. Although improvements could be made by performing multiple measurement cycles and then averaging the results, it is recommended that transient-based methodologies should only be applied when quiescent surface temperature measurements are not available, and/or the underlying assumptions employed in the time-averaged methodologies 
cannot be satisfied.

Results presented from both investigated geometries demonstrate the feasibility of applying infrared thermography as a near-wall diagnostic technique for laminar separation bubbles. Comparison of the characterization achieved in the presented experimental investigations shows the importance of minimizing wall-normal conductive heat flux, as improved characterization precision is achieved on the airfoil geometry where convection is the dominant mode of heat transfer. For such situations where heat transfer is dominated by forced convection, time-averaged measurements of surface temperature acquired in quiescent and flow conditions can be utilized to estimate the variation of convective heat transfer coefficient. Such distributions can then be used to provide reliable estimates of mean separation, transition, and reattachment. 


\section{Chapter 7}

\section{Recommendations}

The following recommendations are made based on the findings of this thesis:

1. The importance of minimizing wall-normal conduction was established when comparing the results obtained between experiments performed on the flat plate and airfoil geometries. As such, it is recommended that the proposed methodologies are applied in situations where forced convection is the dominant heat transfer mode. Otherwise, conductive heat transfer should be explicitly taken into consideration.

2. Throughout this investigation, comparison of the measured surface temperature distributions within the laminar separation bubble with previous experimental investigations was difficult due to the limited experimental-based literature describing heat transfer variation within a laminar separation bubble. This type of experimental characterization can be performed with infrared thermography. However, accurate characterization of the convective heat transfer would require the use of embedded joule heating elements, such that the surface heating could be controlled and uniformly distributed.

3. In order to decrease fluctuations observed in transient convective heat transfer estimates, the transient decay should be measured several times to reduce the adverse influence of random measurement errors. The results from several runs could be averaged, producing a result with reduced random measurement error.

4. To improve the accuracy and precision associated with transient convective heat transfer estimates, a more advanced infrared camera should be implemented. The maximum acquisition frequency which can be consistently achieved with the proprietary Optris Connect software is $1 \mathrm{~Hz}$, whereas more advanced research-grade infrared cameras are capable of acquiring in the $\mathrm{kHz}$ range, at thermal sensitivities on the order of $10 \mathrm{mK}$. An infrared camera with these capabilities would increase the number of data points available to fit with an exponential function, while decreasing the 
thermal fluctuations associated with each temperature measurement.

5. The results presented in this thesis are aimed at improving the validity of using infrared thermography for instantaneously obtaining near-wall flow characteristics on surfaces where laminar separation bubbles are formed. Although the presented experimental investigation was performed in a controlled laboratory setting, the feasibility of applying the proposed methodologies to in-field measurements is of interest. The time-averaged methodologies should be performed when quiescent temperature measurements are available, or non-uniform surface heating can be accurately modelled. Conversely, the transient based methodology should be employed in applications where quiescent surface temperature measurements are not obtainable, or in periodic flows where the results can be averaged. For example, the transient-based methodology could be applied to obtain phase-locked temperature measurements on a wind turbine blade in operation. 


\section{References}

[1] Lissaman, P. B. S. 1983 Low-Reynolds-Number Airfoils. Annual Review of Fluid Mechanics (15), 223-239. DOI. $\hookleftarrow$

[2] Carmichael, B. H. 1981 Low Reynolds Number Airfoil Survey. Tech. rep., NASA, Capistrano Beach, California, pp. 1-106. URL. $\hookleftarrow$

[3] Mueller, T. J. \& Delaurier, J. D. 2003 Aerodynamics of Small Vehicles. Annual Review of Fluid Mechanics 35 (1), 89-111. DOI. $\hookleftarrow$

[4] Horton, H. P. 1968 Laminar Separation Bubbles in Two and Three Dimensional Incompressible Flow. $\mathrm{PhD}$ thesis, University of London. URL. $\hookleftarrow$

[5] Owen, P. R. \& Klanfer, L. 1955 On the Laminar Boundary Layer Separation from the Leading edge of a Thin Aerofoil. Tech. rep. 220, Aeronautical Research Council, pp. 1-28. URL. $\longleftarrow$

[6] Tani, I. 1964 Low-Speed Flows Involving Bubble Separations. Progress in Aerospace Sciences 5, 70-103. DOI. $\hookleftarrow$

[7] Boutilier, M. S. H. \& Yarusevych, S. 2012 Parametric Study of Separation and Transition Characteristics Over an Airfoil at Low Reynolds Numbers. Experiments in Fluids 52 (6), 1491-1506. DOI.

[8] Gerakopulos, R., Boutilier, M. S. H., \& Yarusevych, S. 2010 Aerodynamic Characterization of a NACA 0018 Airfoil at Low Reynolds Numbers. 40th Fluid Dynamics Conference and Exhibit (July), 1-13. DOI. $\hookleftarrow$

[9] Michelis, T., Yarusevych, S., \& Kotsonis, M. 2018 On the Origin of Spanwise Vortex Deformations in Laminar Separation Bubbles. J. Fluid Mech 841, 81-108. DOI. $\leftarrow$

[10] Burgmann, S. \& Schröder, W. 2008 Investigation of the Vortex Induced Unsteadiness of a Separation Bubble via Time-Resolved and Scanning PIV Measurements. Experiments in Fluids 45 (4), 675-691. DOI. $\hookleftarrow$ 
[11] Istvan, M. S. \& Yarusevych, S. 2018 Effects of Free-Stream Turbulence Intensity on Transition in a Laminar Separation Bubble Formed Over an Airfoil. Experiments in Fluids 59 (3), 1-21. DOI.

[12] Watmuff, J. H. 1999 Evolution of a Wave Packet into Vortex Loops in a Laminar Separation Bubble. Journal of Fluid Mechanics 397, 119-169. DOI.

[13] Boutilier, M. S. H. \& Yarusevych, S. 2012 Separated Shear Layer Transition Over an Airfoil at a Low Reynolds Number. Physics of Fluids 24 (8). DOI. $\hookleftarrow$

[14] LeE, T. \& BAsu, S. 1998 Measurement of Unsteady Boundary Layer Developed on an Oscillating Airfoil Using Multiple Hot-Film Sensors. Experiments in Fluids 25 (2), 108-117. DOI. $\hookleftarrow$

[15] Gartenberg, E. \& Roberts, A. S. J. 1991 Twenty-Five Years of Aerodynamic Research with IR Imaging. Thermosense XIII 29 (2). DOI. $\hookleftarrow$

[16] Thomann, H. \& Frisk, B. 1968 Measurement of Heat Transfer With an Infrared Camera. International Journal of Heat and Mass Transfer 11 (5), 819-826. DOI. $\hookleftarrow$

[17] Cardone, G. 2012 IR Heat Transfer Measurements in Hypersonic Plasma Flows. Quantitative InfraRed Thermography Journal 4 (2), 37-41. DOI. $\hookleftarrow$

[18] Braunling, W., Quast, A., \& Dietrichs, H. 1988 Detection of Separation Bubbles by Infrared Images in Transonic Turbine Cascades. Asme Turbo Expo (October 1988). DOI. $\leftarrow$

[19] De Luca, L., Carlomagno, G. M., \& Buresti, G. 1990 Boundary Layer Diagnostics by Means of an Infrared Scanning Radiometer. Experiments in Fluids 9 (3), 121-128. DOI. $\hookleftarrow$

[20] Crawford, B. K., Duncan, G. T., West, D. E., \& Saric, W. S. 2013 LaminarTurbulent Boundary Layer Transition Imaging Using IR Thermography. Optics and Photonics Journal 3 (3), 233-239. DOI. $\hookleftarrow$

[21] Raffel, M. \& Merz, C. B. 2014 Differential Infrared Thermography for Unsteady Boundary-Layer Transition Measurements. AIAA Journal 52 (9), 2090-2093. DOI.

[22] Lang, W., Gardner, A. D., Mariappan, S., Klein, C., \& Raffel, M. 2015 Boundary-Layer Transition on a Rotor Blade Measured by Temperature-Sensitive Paint, Thermal Imaging and Image Derotation. Experiments in Fluids 56 (6), 1-14. DOI. $\hookleftarrow$ 
[23] Dollinger, C., Balaresque, N., Schaffarczyk, A. P., \& Fischer, A. 2016 Thermographic Detection of Separated Flow. Journal of Physics: Conference Series 753 (7). DOI.

[24] Joseph, L. A., Borgoltz, A., \& Devenport, W. 2016 Infrared Thermography for Detection of Laminar-Turbulent Transition in Low-Speed Wind Tunnel Testing. Experiments in Fluids $\mathbf{5 7}$ (5), 1-13. DOI. $\hookleftarrow$

[25] Von Hoesslin, S., Stadlbauer, M., Gruendmayer, J., \& Kähler, C. J. 2017 Temperature Decline Thermography for Laminar-Turbulent Transition Detection in Aerodynamics. Experiments in Fluids 58 (9), 1-10. DOI. $\hookleftarrow$

[26] Szewczyk, M., Smusz, R., De Groot, K., Meyer, J., Kucaba-Pietal, A., \& Rzucidlo, P. 2017 In-Flight Investigations of the Unsteady Behaviour of the Boundary Layer with Infrared Thermography. Measurement Science and Technology 28 (4), 1-16. DOI.

[27] Montelpare, S. \& Ricci, R. 2004 A Thermographic Method to Evaluate the Local Boundary Layer Separation Phenomena on Aerodynamic Bodies Operating at Low Reynolds Number. International Journal of Thermal Sciences 43 (3), 315329. DOI. $\hookleftarrow$

[28] Ricci, R. \& Montelpare, S. 2005 A Quantitative IR Thermographic Method to Study the Laminar Separation Bubble Phenomenon. International Journal of Thermal Sciences 44 (8), 709-719. DOI.

[29] Arcondoulis, E., Doolan, C., Zander, A. C., \& Brooks, L. A. 2011 A Review of Trailing Edge Noise Generated by Airfoils at Low to Moderate Reynolds Number. Acoustics Australia 38 (3), 135-139.

[30] Ol, M., McCauliffe, B., Hanff, E., Scholz, U., \& Kaehler, C. 2005 Comparison of Laminar Separation Bubble Measurements on a Low Reynolds Number Airfoil in Three Facilities. 35th AIAA Fluid Dynamics Conference and Exhibit (June). DOI. $\hookleftarrow$

[31] Lang, M., Rist, U., \& Wagner, S. 2004 Investigations on Controlled Transition Development in a Laminar Separation Bubble by means of LDA and PIV. Experiments in Fluids 36 (1), 43-52. DOI.

[32] Yarusevych, S., Sullivan, P. E., \& Kawall, J. G. 2009 On Vortex Shedding from an Airfoil in Low-Reynolds-Number Flows. Journal of Fluid Mechanics 632, 245. DOI. $\leftarrow$ 
[33] O’Meara, M. \& Mueller, T. J. 1987 Laminar Separation Bubble Characteristics on an Airfoil at Low Reynolds Numbers. AIAA Journal 25 (8), 1033-1041. DOI. $\hookleftarrow$

[34] Pucher, P. \& Gohl, R. 1987 Experimental Investigation of Boundary Layer Separation With Heated Thin-Film Sensors. Journal of Turbomachinery 109 (2), 303309. DOI. $\leftarrow$

[35] Lou, W. \& Hourmouziadis, J. 2000 Separation Bubbles Under Steady and Periodic-Unsteady Main Flow Conditions. In 45th ASME International Gas Turbine $\&$ Aeroengine Technical Congress, number 122, pp. 2-13, Munich, Germany. DOI. $\hookleftarrow$

[36] Haggmark, C. P., Bakchinov, A. A., \& Alfredsson, P. H. 2000 Experiments on a Two-Dimensional Laminar Separation Bubble. Philosophical Transactions of the Royal Society A: Mathematical, Physical and Engineering Sciences 358 (1777), 3193-3205. DOI. $\leftarrow$

[37] Marxen, O., Lang, M., Rist, U., \& Wagner, S. 2003 A Combined Experimental/Numerical Study of Unsteady Phenomena in a Laminar Separation Bubble. Flow, Turbulence and Combustion 71 (1-4), 133-146. DOI.

[38] Yarusevych, S., Sullivan, P. E., \& Kawall, J. G. 2006 Coherent Structures in an Airfoil Boundary Layer and Wake at Low Reynolds Numbers. Physics of Fluids 18 (4). DOI.

[39] Radespiel, R. E., Windte, J., \& Scholz, U. 2007 Numerical and Experimental Flow Analysis of Moving Airfoils with Laminar Separation Bubbles. AIAA Journal 45 (6), 1346-1356. DOI.

[40] Yarusevych, S., Sullivan, P. E., \& Kawall, J. G. 2007 Effect of Acoustic Excitation Amplitude on Airfoil Boundary Layer and Wake Development. AIAA Journal 45 (4), 760-771. DOI. $\hookleftarrow$

[41] Yarusevych, S., Sullivan, P. E., \& Kawall, J. G. 2009 Smoke-Wire Flow Visualization in Separated Flows at Relatively High Velocities. AIAA Journal 47 (6), 1592-1595. DOI. $\hookleftarrow$

[42] Alam, M. \& Sandham, N. D. 2000 Direct Numerical Simulation of 'Short' Laminar Separation Bubbles with Turbulent Reattachment. Journal of Fluid Mechanics 410, $1-28$. DOI. $\hookleftarrow$

[43] BRILEy, W. R. 1970 A Numerical Study of Laminar Separation Bubbles Using the Navier-Stokes Equations. Journal of Fluid Mechanics 47 (4), 713-736. DOI. $\hookleftarrow$ 
[44] Pauley, L. L., Moin, P., \& Reynolds, W. C. 1990 The structure of twodimensional separation. J. Fluid Mech 220, 397-411. DOI. $\hookleftarrow$

[45] Rist, U. \& Maucher, U. 2002 Investigations of Time-Growing Instabilities in Laminar Separation Bubbles. European Journal of Mechanics, B/Fluids 21 (5), 495-509. DOI. $\leftarrow$

[46] Wissink, J. G., Michelassi, V., \& Rodi, W. 2004 Heat Transfer in a Laminar Separation Bubble Affected by Oscillating External Flow. International Journal of Heat and Fluid Flow 25 (5), 729-740. DOI.

[47] Roberts, S. K. \& Yaras, M. I. 2005 Large-Eddy Simulation of Transition in a Separation Bubble. Journal of Fluids Engineering 128 (2), 232-238. DOI.

[48] Jones, L. E., Sandberg, R. D., \& Sandham, N. D. 2008 Direct Numerical Simulations of Forced and Unforced Separation Bubbles on an Airfoil at Incidence. Journal of Fluid Mechanics 602, 175-207. DOI.

[49] Almutairi, J. H., Jones, L. E., \& Sandham, N. D. 2010 Intermittent Bursting of a Laminar Separation Bubble on an Airfoil. AIAA Journal 48 (2), 414-426. DOI. $\hookleftarrow$

[50] Cadieux, F. \& Domaradzki, J. A. 2014 Direct Numerical Simulation and Large Eddy Simulation of Laminar Separation Bubbles at Moderate Reynolds Numbers. Journal of Fluids Engineering 136 (6), 1-5. DOI.

[51] Yang, Z. \& Voke, P. R. 2001 Large-Eddy Simulation of Boundary-Layer Separation and Transition at a Change of Surface Curvature. Journal of Fluid Mechanics 439, 305-333. DOI. $\leftarrow$

[52] Gaster, M. 1967 The Structure and Behaviour of Laminar Separation Bubbles. Aeronautical Research Council Reports and Memoranda (3595), 1-31. ISBN. $\leftarrow$

[53] Kurelex, J. W. 2016 Transition in a Laminar Separation Bubble and the Effect of Acoustic Excitation. MASc. Thesis, University of Waterloo. URL. $\hookleftarrow$

[54] Kirk, T. M. \& Yarusevych, S. 2017 Vortex Shedding Within Laminar Separation Bubbles Forming Over an Airfoil. Experiments in Fluids 58 (5), 1-17. DOI. $\hookleftarrow$

[55] Kurelek, J. W., Lambert, A. R., \& Yarusevych, S. 2016 Coherent Structures in the Transition Process of a Laminar Separation Bubble. AIAA Journal 54 (8), 2295-2309. DOI. $\hookleftarrow$

[56] Kurelek, J. W., Kotsonis, M., \& Yarusevych, S. 2018 Transition in a Separation Bubble Under Tonal and Broadband Acoustic Excitation. Journal of Fluid Mechanics 853, 1-36. DOI. 
[57] Saric, W. S., Reed, H. L., \& Kerschen, E. J. 2002 Boundary-Layer Receptivity to Freestream Disturbances. Annual Review of Fluid Mechanics 34 (1996), 291-319. DOI. $\leftarrow$

[58] Tani, I. 1969 Boundary-Layer Transition. Annual Review of Fluid Mechanics 1 (1), $169-196 . \hookleftarrow$

[59] Dovgal, A. V., Kozlov, V. V., \& Michalke, A. 1994 Laminar Boundary Layer Separation: Instability and Associated Phenomena. Progress in Aerospace Sciences 30 (1), 61-94. DOI.

[60] Rist, U., Maucher, U., \& Wagner, S. 1996 Direct Numerical Simulation of Some Fundamental Problems Related to Transition in Laminar Separation Bubbles. Proceedings of the ECCOMAS Computational Fluid Dynamics Conference 319-325. URL. $\hookleftarrow$

[61] Bellows, W. J. \& Mayle, R. E. 1986 Leading Edge Separation Bubble. Journal of Turbomachinery 108 (1), 131-136. DOI.

[62] Zhong, S. 2002 Detection of Flow Separation and Reattachment Using ShearSensitive Liquid Crystals. Experiments in Fluids 32 (6), 667-673. DOI.

[63] Gerakopulos, R. \& Yarusevych, S. 2012 Novel Time-Resolved Pressure Measurements on an Airfoil at a Low Reynolds Number. AIAA Journal 50 (5), 11891200. DOI. $\hookleftarrow$

[64] Istvan, M. S., Kurelek, J. W., \& Yarusevych, S. 2017 Turbulence Intensity Effects on Laminar Separation Bubbles Formed over an Airfoil. AIAA Journal 56 (4), 1-13. DOI. $\hookleftarrow$

[65] Yarusevych, S., Kawall, J. G., \& Sullivan, P. E. 2008 Unsteady Separated Flow Characterization on Airfoils Using Time-Resolved Surface Pressure Measurements. AIAA Journal 46 (2), 508-516. DOI. $\hookleftarrow$

[66] Sullivan, J., Liu, T., Campbell, B., \& Burns, S. 1997 Temperature and Pressure Sensitive Luminescent Paints in Aerodynamics. Applied Mechanics Reviews 50 (4), 227-246. DOI. $\hookleftarrow$

[67] Hilfer, M., Dufhaus, S., Petersen, A., Yorita, D., \& Klein, C. 2014 Application of Pressure- and Temperature-Sensitive Paint on a Highly Loaded Turbine Guide Vane in a Transonic Linear Cascade. In 1st Global and Propulsion Forum, pp. 2-11, Zurich, Switzerland. URL.

[68] Liu, T. 2010 Pressure- and Temperature-Sensitive Paints. Encyclopedia of Aerospace Engineering 1-11. DOI. 
[69] Boutilier, M. S. H. \& Yarusevych, S. 2014 Influence of Hot-Wire Probe and Traverse on Low-Reynolds-Number Airfoil Experiments. AIAA Journal 52 (11), 2618-2622. DOI. $\leftarrow$

[70] Hain, R., Kahler, C. J., \& Radespiel, R. E. 2009 Dynamics of laminar separation bubbles at low-Reynolds-number aerofoils. J . Fluid Mech 630, 129-153. DOI.

[71] Serna, J. \& LÁzaro, B. J. 2014 The Final Stages of Transition and the Reattachment Region in Transitional Separation Bubbles. Experiments in Fluids 55 (4). DOI. $\hookleftarrow$

[72] Pröbsting, S. \& Yarusevych, S. 2015 Laminar Separation Bubble Development on an Airfoil Emitting Tonal Noise. Journal of Fluid Mechanics 780, 167-191. DOI. $\hookleftarrow$

[73] Yarusevych, S. \& Kotsonis, M. 2017 Steady and transient response of a laminar separation bubble to controlled disturbances. Journal of Fluid Mechanics 813, 955990. DOI. $\hookleftarrow$

[74] Michelis, T., Yarusevych, S., \& Kotsonis, M. 2017 Response of a Laminar Separation Bubble to Impulsive Forcing. Journal of Fluid Mechanics 820, 633-666. DOI. $\hookleftarrow$

[75] Brendel, M. \& Mueller, T. J. 1987 Boundary-Layer Measurements on an Airfoil at Low Reynolds Numbers. Journal of Aircraft 25 (7), 612-617. DOI.

[76] Liu, T., Campbell, B. T., \& Sullivan, J. P. 1995 Fluorescent Paint for Measurement of Heat Transfer in Shock-Turbulent Boundary Layers. Experimental Thermal and Fluid Science 10 (1), 101-112. DOI.

[77] LiU, T. 1994 Temperature Sensitive Fluorescent Paint System. In 18th AIAA Aerospace Ground Testing Conference, pp. 1-19. DOI. $\leftarrow$

[78] Ristic, S. 2007 Flow Visualisation Techniques in Wind Tunnels Part I - Non optical Methods. Scientific Technical Review 57 (1), 39-49. URL.

[79] Miozzi, M., Capone, A., Costantini, M., Fratto, L., Klein, C., \& Di Felice, F. 2019 Skin Friction and Coherent Structures Within a Laminar Separation Bubble. Experiments in Fluids $\mathbf{6 0}(1), 13$. DOI. $\hookleftarrow$

[80] LiU, T. \& Woodiga, S. 2011 Feasibility of Global Skin Friction Diagnostics Using Temperature Sensitive Paint. Measurement Science and Technology 22, 1-11. DOI. 
[81] Miozzi, M., Capone, A., Costantini, M., Klein, C., \& Felice, F. D. 2018 Coherent Structures Within the Laminar Separation Bubble on a NACA $0015 \mathrm{Hy}-$ drofoil. In 5th International Conference on Experimental Fluid Mechanics, number July, Munich, Germany. URL. $\hookleftarrow$

[82] Quast, A. 1987 Detection of Transition by Infrared Image Techniques. Technical Soaring 30 (1), 33-38. URL.

[83] Dollinger, C., Sorg, M., Balaresque, N., \& Fischer, A. 2018 Measurement Uncertainty of IR Thermographic Flow Visualization Measurements for Transition Detection on Wind Turbines in Operation. Experimental Thermal and Fluid Science 97, 279-289. DOI.

[84] Dollinger, C., Balaresque, N., Gaudern, N., Gleichauf, D., Sorg, M., \& Fischer, A. 2019 IR Thermographic Flow Visualization for the Quantification of Boundary Layer Flow Disturbances Due to the Leading Edge Condition. Renewable Energy 138, 709-721. DOI.

[85] Costantini, M., Hein, S., Henne, U., Klein, C., Koch, S., \& Schojda, L. 2016 Pressure Gradient and Nonadiabatic Surface Effects on Boundary Layer Transition. AIAA Journal 54 (11), 3465-3480. DOI.

[86] Meola, C. \& Carlomagno, G. M. 2004 Recent Advances in the Use of Infrared Thermography. Measurement Science and Technology 15 (9), R27-R58. DOI.

[87] Simeonides, G., Vermeulen, J., Boerrigter, H. L., \& Wendt, J. F. 1993 Quantitative Heat Transfer Measurements in Hypersonic Wind Tunnels by Means of Infrared Thermography. IEEE Transactions on Aerospace and Electronic Systems 29 (July), 878-893. DOI.

[88] Banks, D. W., van Dam, C. P., Shiu, H. J., \& Miller, G. M. 2000 Visualization of In-Flight Flow Phenomena Using Infrared Thermography. In 9th International Symposium on Flow Visualization, number July. URL. $\hookleftarrow$

[89] Raffel, A. D., Gardner, C. C., \& Wolf, M. 2016 A new method of dynamic and static stall detection using infrared thermography. In Experiments in Fluids, number September, pp. 1-13. DOI.

[90] Simon, B., Filius, A., Tropea, C., \& Grundmann, S. 2016 IR Thermography for Dynamic Detection of Laminar-Turbulent Transition. Experiments in Fluids $\mathbf{5 7}$ (5), 1-12. DOI. 
[91] Richter, K., Wolf, C. C., Gardner, A. D., \& Merz, C. B. 2016 Detection of Unsteady Boundary Layer Transition Using Three Experimental Methods. In 54th AIAA Aerospace Sciences Meeting, number January, pp. 1-22, San Diego, California. DOI. $\leftarrow$

[92] Gardner, A. D., Eder, C., Wolf, C. C., \& Raffel, M. 2017 Analysis of Differential Infrared Thermography for Boundary Layer Transition Detection. Experiments in Fluids 58 (9), 1-14. DOI.

[93] Drela, M. 1989 XFOIL: An Analysis and Design System for Low Reynolds Number Airfoils. In Low Reynolds Number Airfoil Aerodynamics, pp. 1-12. Springer Berlin Heidelberg. DOI. $\hookleftarrow$

[94] Dollinger, C., Balaresque, N., Sorg, M., \& Fischer, A. 2018 IR Thermographic Visualization of Flow Separation in Applications with Low Thermal Contrast. Infrared Physics \& Technology 88, 254-264. DOI.

[95] Newton, I. 1701 VII. Scala Graduum Caloris. Royal Society of London 22 (170), 824-829. URL. $\hookleftarrow$

[96] Kestin, J. \& Richardson, P. D. 1963 Heat Transfer Across Turbulent, Incompressible Boundary Layers. International Journal of Heat and Mass Transfer 6 (2), 147-189. DOI.

[97] Schlichting, H. 1960 Boundary Layer Theory. ninth ed. Springer, p. 647. DOI. $\hookleftarrow$

[98] Simonson, J. R. 1988 Engineering Heat Transfer. 2nd ed. Macmillan Education Ltd, pp. 1-261. DOI.

[99] Reynolds, O. 1874 On the Extent and Action of the Heating Surface of Steam Boilers. Proceedings of the Literary and Philosophical Society of Manchester 14 (5), 7-12. URL. $\hookleftarrow$

[100] Lin, N., Reed, H. L., \& SARIC, W. S. 1992 Effect of Leading-Edge Geometry on Boundary Layer Receptivity to Freestream Sound. In Instability, Transition, and Turbulence, part 42, pp. 421-440. Springer. DOI. $\hookleftarrow$

[101] Boutilier, M. S. H. 2011 Experimental Investigation of Transition over a NACA 0018 Airfoil at a Low Reynolds Number. MASc. Thesis, University of Waterloo. URL. $\hookleftarrow$

[102] Istvan, M. S. 2016 Effects of Free-Stream Turbulence Intensity on Laminar Separation Bubbles. MASc. Thesis, University of Waterloo. URL. $\hookleftarrow$ 
[103] Boutilier, M. S. H. \& Yarusevych, S. 2012 Effects of End Plates and Blockage on Low-Reynolds-Number Flows Over Airfoils. AIAA Journal 50 (7), 1547-1559. DOI. $\hookleftarrow$

[104] Scarano, F. \& Riethmuller, M. L. 2000 Advances in Iterative Multigrid PIV Image Processing. Experiments in Fluids 29 (1), 51-60.

[105] Meinhart, C. D., Wereley, S. T., \& Santiago, J. G. 2000 A PIV Algorithm for Estimating Time-Averaged Velocity Fields. Journal of Fluids Engineering 122 (June 2000), 285-289. DOI. $\hookleftarrow$

[106] Smith, M. C. \& Kuethe, A. M. 1966 Effects of Turbulence on Laminar Skin Friction and Heat Transfer. The Physics of Fluids 9 (12), 2337-2344. DOI. $\hookleftarrow$

[107] Mayle, R. E., Blair, M. F., \& Kopper, F. C. 1979 Turbulent Boundary Layer Heat Transfer on Curved Surfaces. Journal of Heat Transfer 101 (3), 521-525. DOI.

[108] Guo, Z. Y., Li, D. Y., \& Wang, B. X. 1998 A Novel Convept for Convective Heat Transfer Enhancement. International Journal of Heat and Mass Transfer 41 (14), 2221-2225. DOI. $\hookleftarrow$

[109] Kestin, J., Maeder, P. F., \& Wang, H. E. 1961 Influence of Turbulence on the Transfer of Heat from Plates with and Without a Pressure Gradient. International Journal of Heat and Mass Transfer 3, 133-154. DOI. $\hookleftarrow$

[110] Junkhan, G. H. \& Serovy, G. K. 1967 Effects of Free-Stream Turbulence and Pressure Gradient on Flat- Plate Boundary-Layer Velocity Profiles and on Heat Transfer. Journal of Heat Transfer 89 (2), 169-175. DOI. $\hookleftarrow$

[111] Houra, T. \& Nagano, Y. 2006 Effects of Adverse Pressure Gradient on Heat Transfer Mechanism in Thermal Boundary Layer. International Journal of Heat and Fluid Flow 27, 967-976. DOI. $\hookleftarrow$

[112] Ghasemi, E., Mceligot, D. M., Nolan, K. P., Crepeau, J., Siahpush, A., Budwig, R. S., \& Tokuhiro, A. 2014 Effects of Adverse and Favorable Pressure Gradients on Entropy Generation in a Transitional Boundary Layer Region Under the Influence of Freestream Turbulence. International Journal of Heat and Mass Transfer 77, 475-488. DOI.

[113] Rivir, R. B., Johnston, J. P., \& Eaton, J. K. 1992 Heat Transfer on a Flat Surface Under a Region of Turbulent Separation. In International Gas Turbine and Aeroengine Congress and Exposition, pp. 1-6. URL. $\hookleftarrow$ 
[114] Spalart, P. R. \& Strelets, M. K. 2000 Mechanisms of Transition and Heat Transfer in a Separation Bubble. Journal of Fluid Mechanics 403, 329-349. DOI. $\hookleftarrow$

[115] Selig, M. S., Deters, R. W., \& Williamson, G. A. 2011 Wind Tunnel Testing Airfoils at Low Reynolds Numbers. 49th AIAA Aerospace Sciences Meeting (January). DOI. $\hookleftarrow$

[116] Durand, W. F. 1934 Application of Conformal Transformation to Fields of Flow. In Aerodynamic Theory, pp. 171-185. Springer Berlin Heidelberg. DOI. $\hookleftarrow$

[117] Jambunathan, K., Lai, E., Moss, M. A., \& Button, B. L. 1992 A Review of Heat Transfer Data for Single Circular Jet Impingement. International Journal of Heat and Fluid Flow 13 (2), 106-115. DOI.

[118] OтA, T. 2000 A Survey of Heat Transfer in Separated and Reattached Flows. Applied Mechanics Reviews 53 (8), 219-235. DOI.

[119] Seban, R. A. 1959 Heat Transfer to Separated and Reattached Subsonic Turbulent Flows Obtained Downstream of a Surface Step. Journal of the Aerospace Sciences 26 (12), 809-814. DOI.

[120] Cooper, T. E., Field, R. J., \& Meyer, J. F. 1975 Liquid Crystal Thermography and its Application to the Study of Convective Heat Transfer. Journal of Heat Transfer 97 (3), 442-450. DOI.

[121] Shan, H., Jiang, L., \& Liv, C. 2005 Direct Numerical Simulation of Flow Separation Around a NACA 0012 Airfoil. Computers and Fluids 34 (9), 1096-1114. DOI. $\hookleftarrow$

[122] Incropera, F., DeWitt, D., Bergman, T., \& Lavine, A. 2007 Fundamentals of Heat and Mass Transfer. sixth edit ed. John Wiley \& Sons, pp. 1-916. ISBN. $\hookleftarrow$

[123] Moffat, R. J. 1982 Contributions to the Theory of Single-Sample Uncertainty Analysis. Journal of Fluids Engineering 104 (2), 250-258. DOI.

[124] Moffat, R. J. 1985 Using Uncertainty Analysis in the Planning of an Experiment. Journal of Fluids Engineering 107 (2), 173-178. DOI.

[125] Moffat, R. J. 1988 Describing the Uncertainties in Experimental Results. Experimental Thermal and Fluid Science 1, 3-17. DOI.

[126] Sciacchitano, A., Wieneke, B., \& Scarano, F. 2013 PIV Uncertainty Quantification by Image Matching. Measurement Science and Technology 24,1-16. DOI. $\hookleftarrow$ 
Appendices 


\section{Appendix A}

\section{Supplementary Results}

The results presented in Chapters 4 and 5 highlighted the main findings of both experimental investigations. However, in order to keep the discussion concise, a subset of the data collected could not be presented in the thesis body. Such results are presented here, in the order of which they are discussed in Chapters 4 and 5 , to provide the reader with the entire dataset collected throughout the experimental investigations.

\section{A.1 Flat Plate Data}

Measurements presented in Chapter 4 were focused for Reynolds numbers $\operatorname{Re}_{L}=27,000$, $\operatorname{Re}_{L}=36,000$, and $\operatorname{Re}_{L}=45,000$. Due to the relative simplicity of surface pressure measurements, data were measured at a number of supplementary flow conditions, as shown in Table 3.1. These results provided initial estimates of the location and size of the separated region. Distributions of $C_{P}$ are shown for each condition in Fig. A.1, with locations of mean separation, transition, and reattachment identified with circle, diamond, and square markers, respectively. These locations are summarized in Fig. A.4, where trends of the laminar separation bubble due to changes in Reynolds number can be visualized.

It is mentioned in Section 4.1 that a nearly linear trend is observed between the maximum displacement thickness and total streamwise bubble extent as measured with PIV. This is shown in Fig. A.2, where the ratio of maximum displacement thickness to total streamwise extent of the separation bubble is plotted. The data points are fit with a linear function, which is shown to pass through the three data points, agreeing with previous experimental investigations $[10,33]$.

The estimated distributions of $h^{*}$ presented in Section 4.2 were calculated with surface temperature distributions which assumed thermal equilibrium in quiescent and flow conditions. In order the validate this assumption, the surface temperature was recorded at 

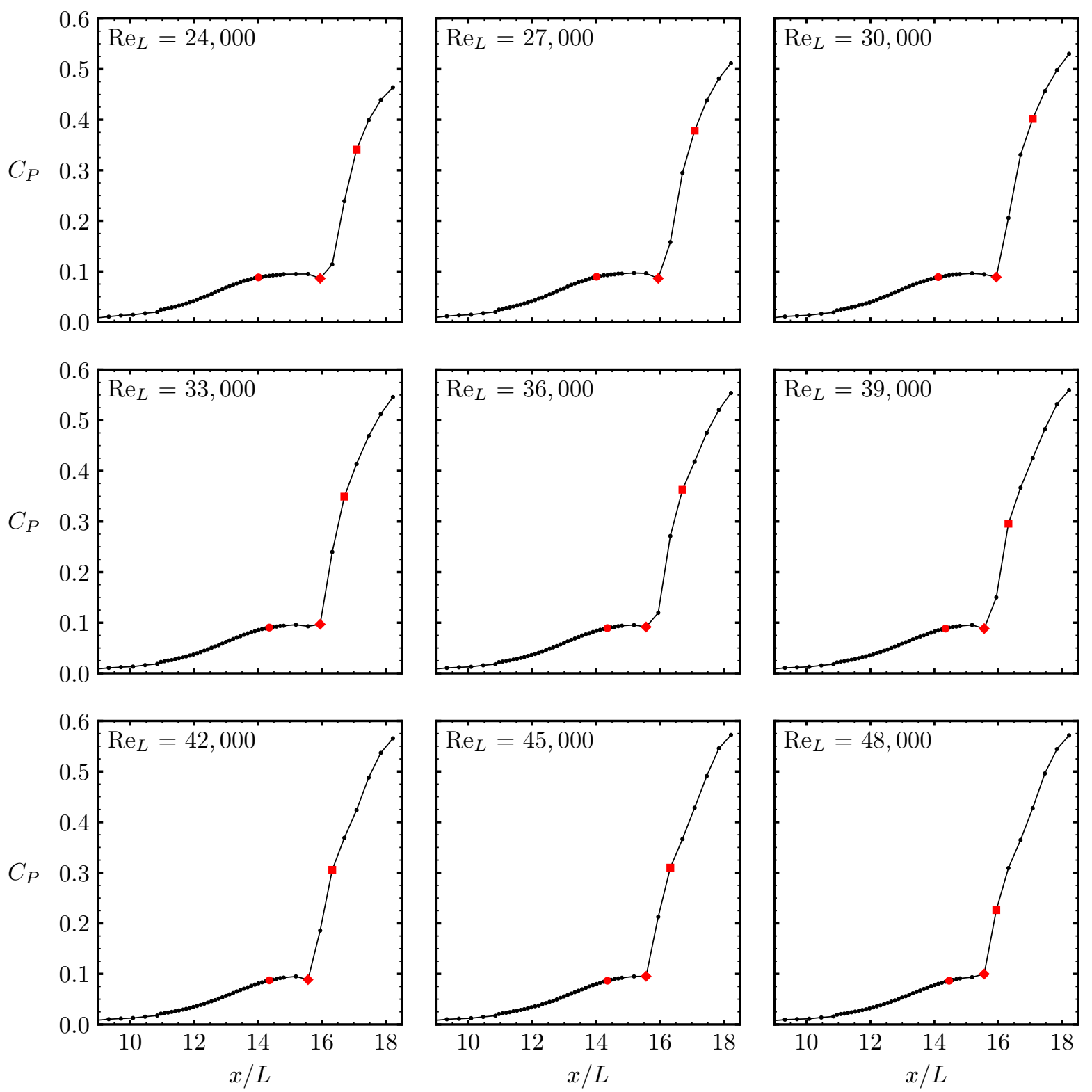

Figure A.1: Measured surface pressure distribution on the flat plate at each investigated flow condition. Circle, diamond, and square markers indicate estimated locations of separation, transition, and reattachment, respectively. 


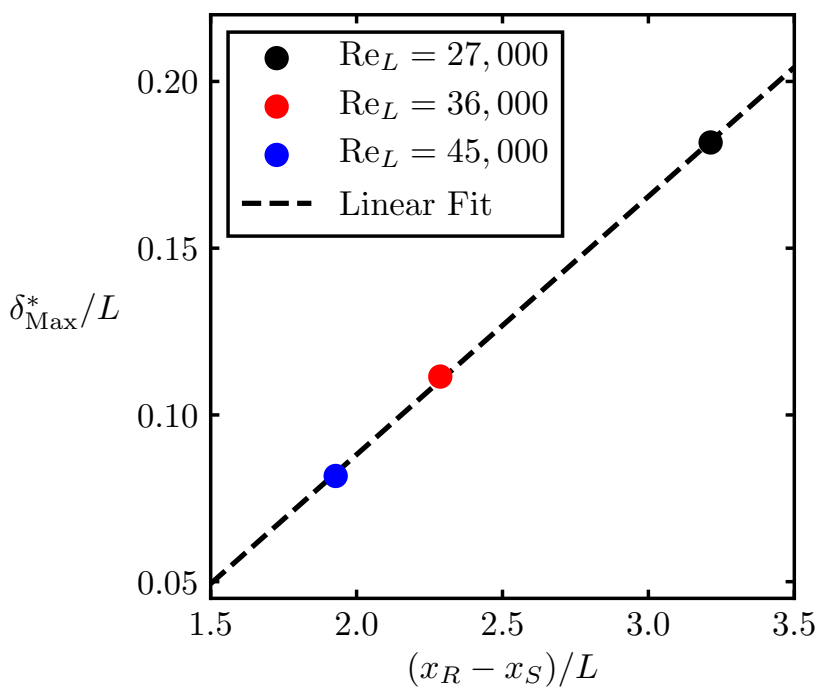

Figure A.2: Linearity shown between maximum displacement thickness and total separation bubble length measured with PIV in flat plate experiments.

$15 \mathrm{~s}$ intervals while the flat plate was continuously heated with the halogen lamps. The transient development of surface temperature at specified streamwise locations is shown in Fig. A.3. The surface temperature is shown to rapidly increase during the initial transient before asymptotically approaching a steady state temperature. The surface temperature is shown to increase less than $1^{\circ} \mathrm{C}$ between $t=120 \mathrm{~min}$ and $t=180 \mathrm{~min}$, allowing for a reasonable assumption of thermal equilibrium. In this regard, measurements of quiescent surface temperature were initiated after the $t=180 \mathrm{~min}$ warm-up period.

Just as measurements of surface pressure were acquired at a number of additional flow conditions, so too were measurements of time-averaged surface temperature. Shown in Fig. A.4a are distributions of surface temperature across all investigated Reynolds numbers, with the corresponding streamwise gradients shown in Fig. A.4b. Using these measurements of time-averaged surface temperature and the mean quiescent surface temperature shown in Fig. 4.12, the variation of $h^{*}$ was computed at each investigated flow condition. Distributions of $h^{*}$ are shown for each investigated Reynolds number in Fig. A.5a, with the corresponding streamwise gradients shown in Fig. A.5b.

Using the distributions of surface pressure, surface temperature, and $h^{*}$ shown in Figs. A.1, A.4, and A.5, the characteristic locations were estimated at each flow condition, following the methodologies discussed in Sections 4.1 and 4.2. The estimated locations of mean separation, transition, and reattachment are compared between each technique in Fig. A.6. Estimations based on time-averaged surface pressure are shown to agree with the generally established trends associated with laminar separation bubbles formed on a flat 


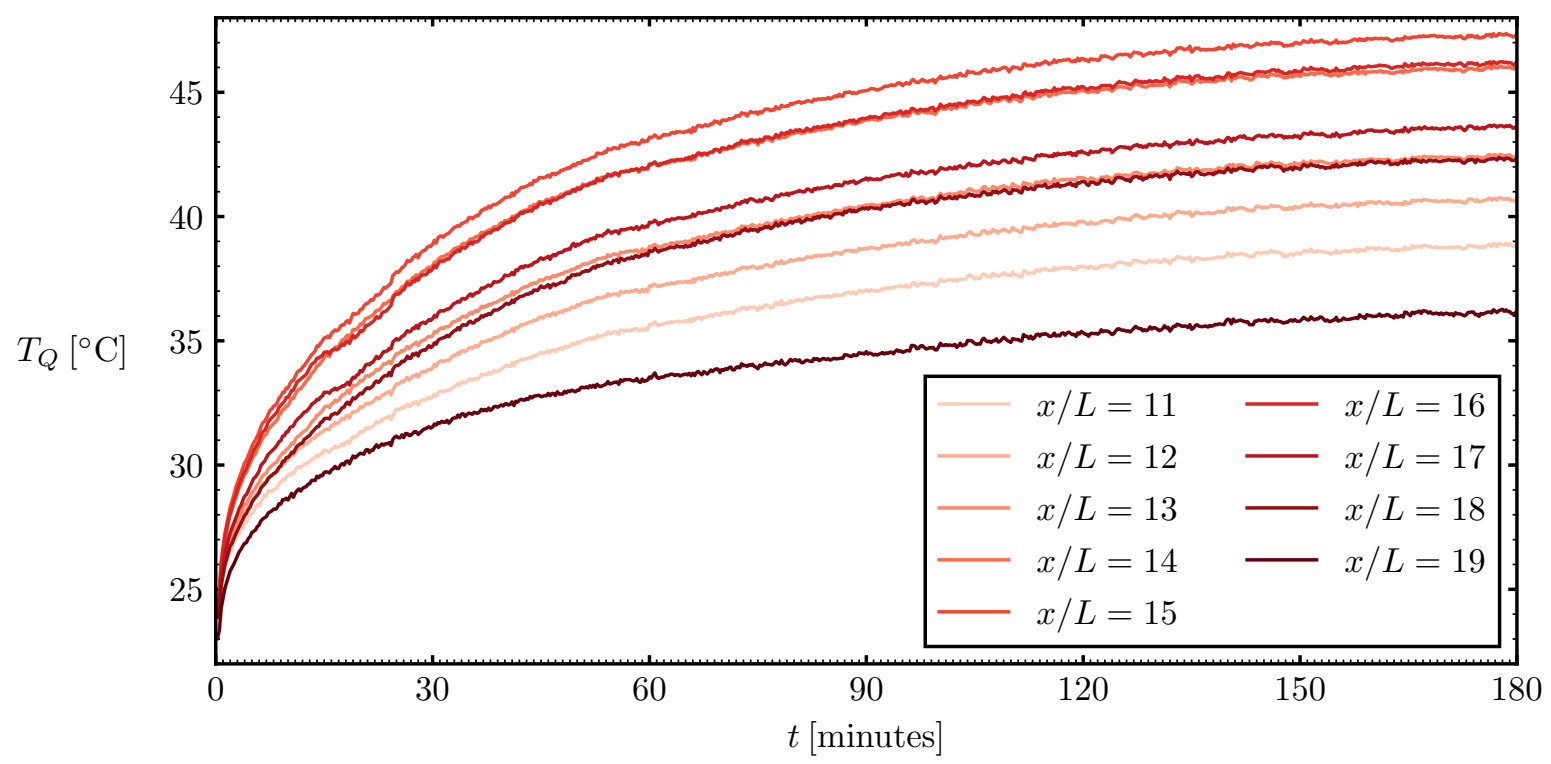

Figure A.3: Transient surface temperature development with the onset of radiative heating in quiescent conditions shown at specified streamwise locations on the flat plate.

plate subject to an increase in Reynolds number. More specifically, the mean separation location is shown to shift downstream, while transition and reattachment advance in the upstream direction. However, limited spatial resolution of the embedded pressure taps results in a discrete movement of the mean transition and reattachment locations. The locations of mean separation and transition established from distributions of surface temperature and $h^{*}$ are shown to generally agree with those predicted from surface pressure distributions. However, the mean separation location estimated from these distributions is identified at a weakly defined extrema in the streamwise gradient, and is therefore subject to a lower estimation precision. This is particularly evident in the estimated separation location from surface temperature distributions at $\operatorname{Re}_{L}=48,000$, where the decreased precision results in an estimated location significantly upstream of that estimated with surface pressure. The locations of mean reattachment estimated from mean surface temperature distributions remain relatively unchanged with increases in Reynolds number due to the region of temperature plateau observed in the after portion of the bubble (Fig. A.4). Although a more pronounced peak convective heat transfer coefficient is observed in distributions of $h^{*}$ (Fig. A.5), the reattachment location estimated from distributions of $h^{*}$ is also shown to remain relatively unchanged with increasing Reynolds number. Thus, signifying that the reattachment location estimated from $h^{*}$ is associated with relatively large bounds of methodological uncertainty. 

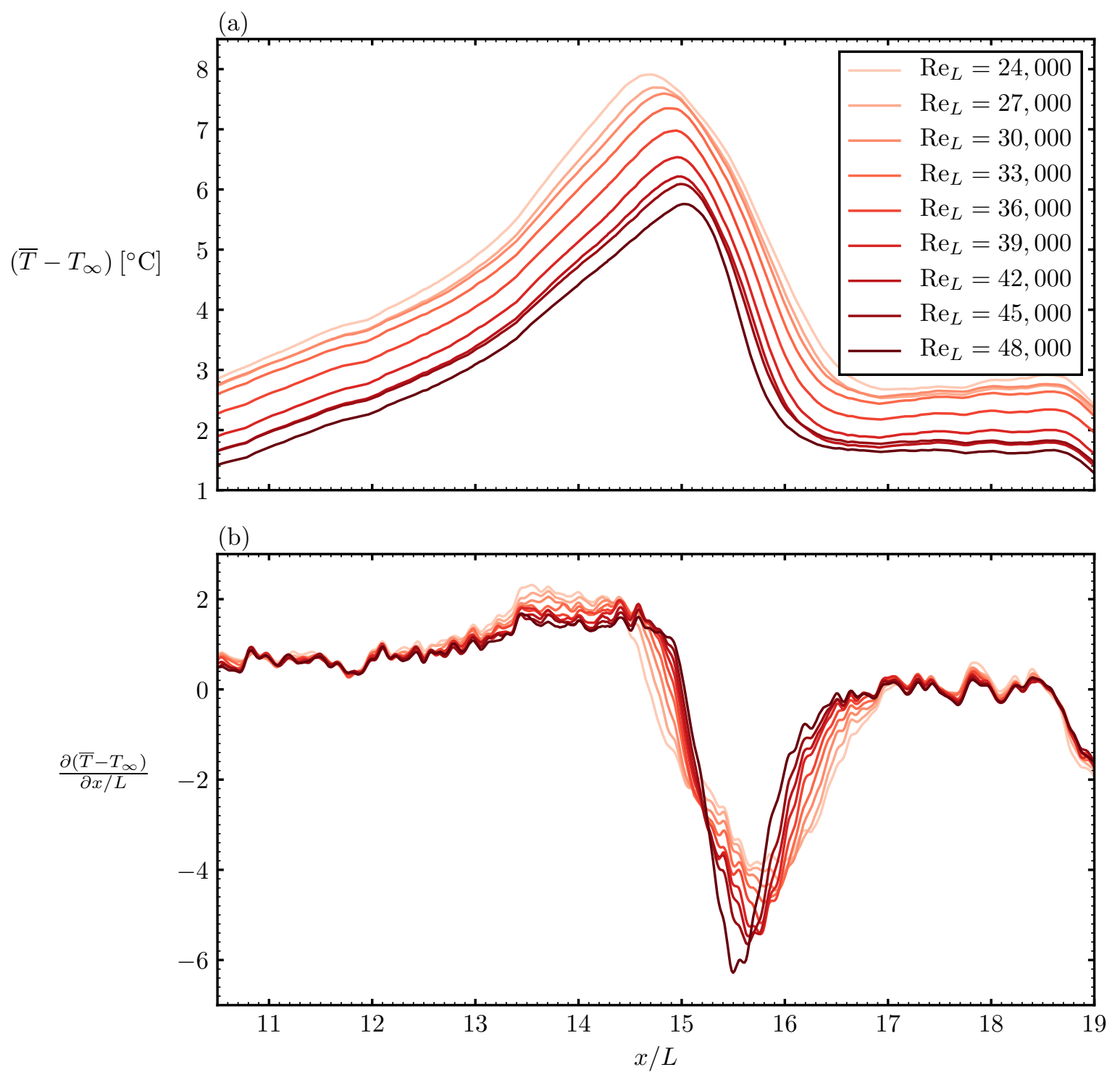

Figure A.4: Time- and spanwise-averaged distributions of (a) surface temperature, and (b) streamwise temperature gradient for each investigated experimental condition on the flat plate. 

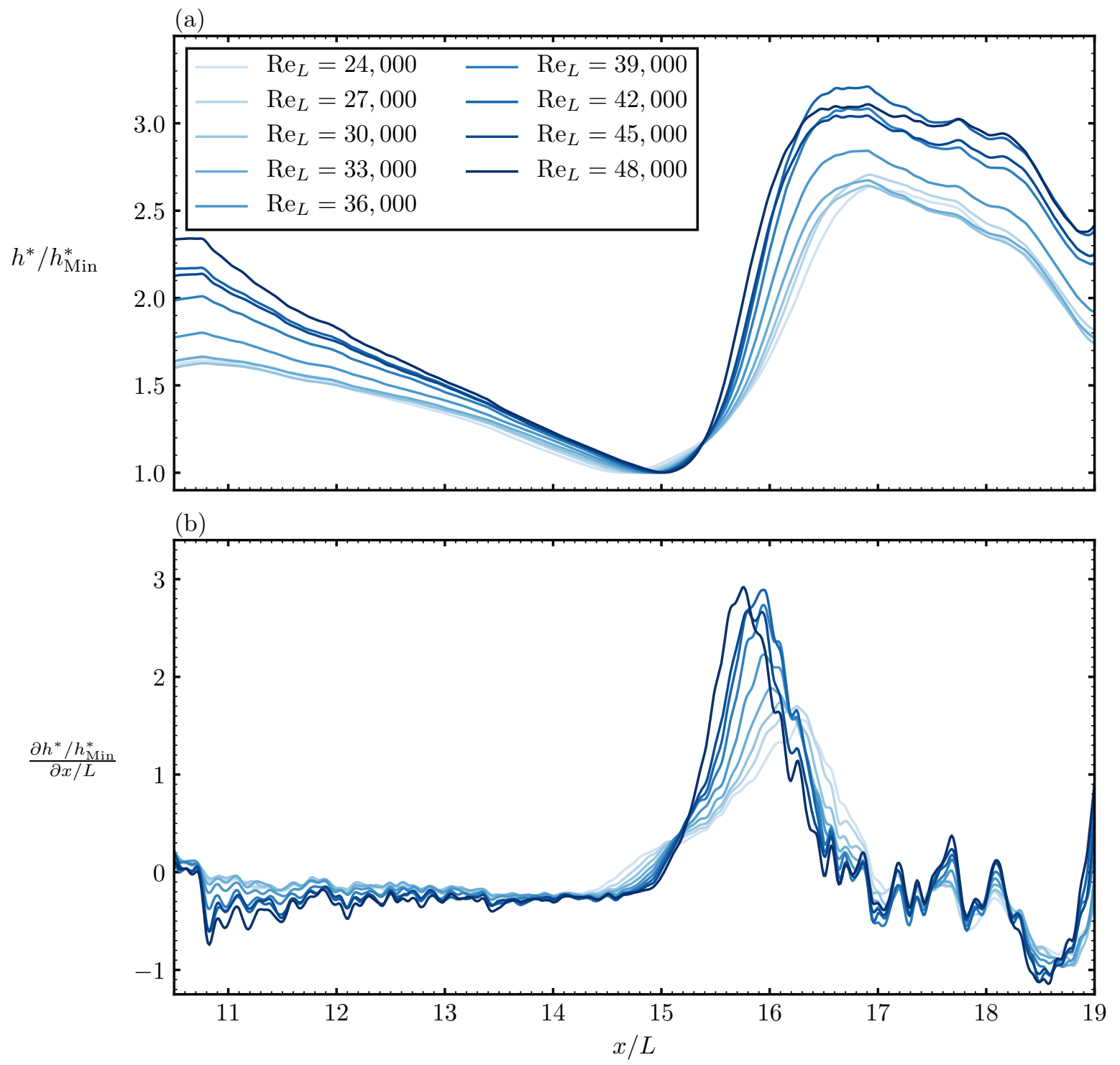

Figure A.5: Time- and spanwise-averaged distributions of (a) $h^{*}$, and (b) its streamwise gradient for each investigated experimental condition on the flat plate. 


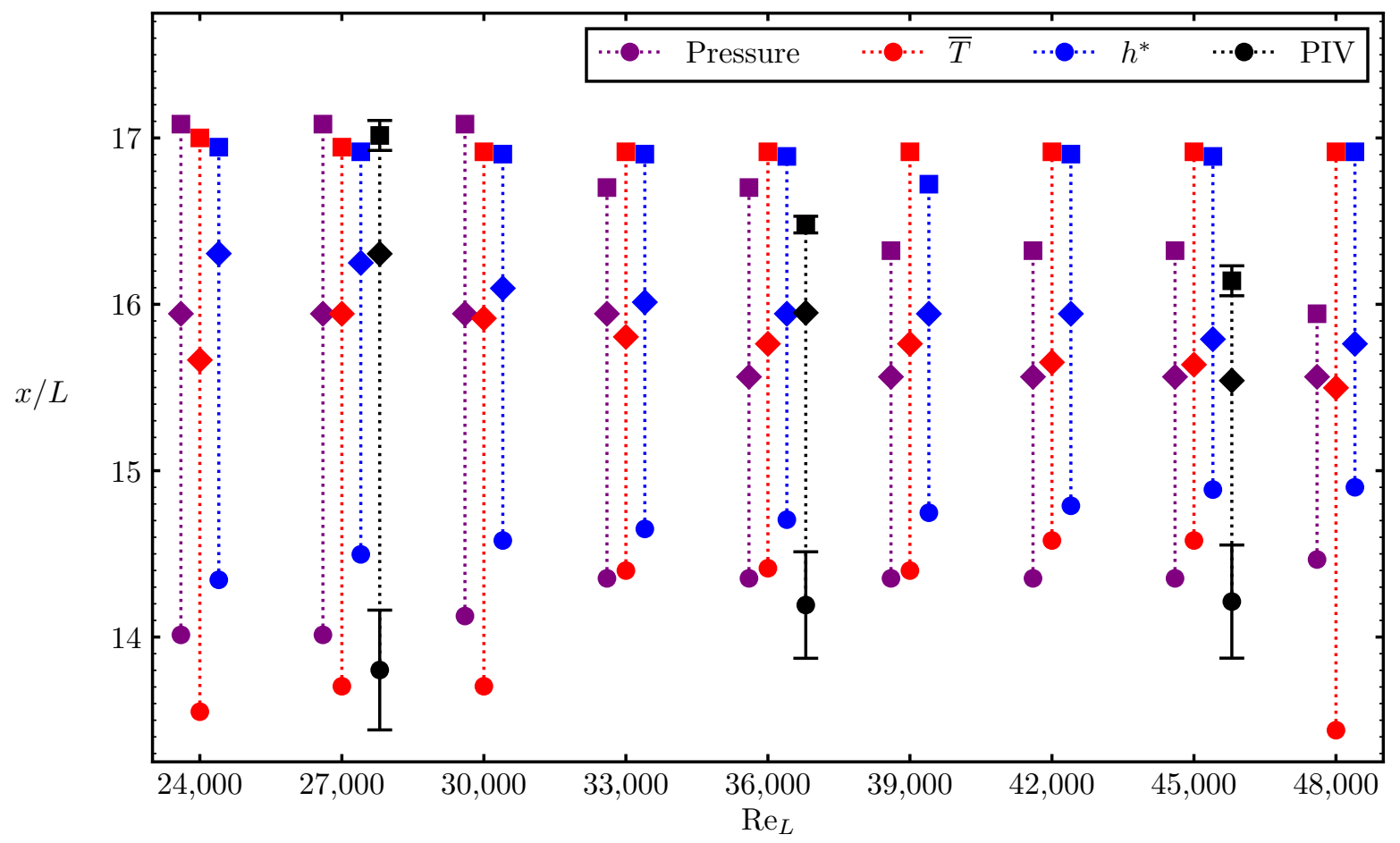

Figure A.6: Locations of mean separation, transition, and reattachment estimated from distributions of surface pressure, surface temperature, and $h^{*}$, at each flat plate flow condition. Separation, transition, and reattachment locations are indicated with circle, diamond, and square markers, respectively. A slight offset from the measurement Reynolds number is applied for visual clarity.

\section{A.2 Airfoil Data}

Just as with the experiments performed on the flat plate, the transient development of surface temperature while the airfoil model was being heated by the halogen lamps was investigated. Shown in Fig. A.7 is the temperature at specified streamwise locations over a period of $120 \mathrm{~min}$. The radiative heating is shown to rapidly increase the surface temperature during the initial transient, with slower temperature increase observed at higher times. As the temperature reaches a relatively constant value, thermal equilibrium can be assumed at the surface. A time of 120 min was employed before measurements were initiated.

As established in Table 3.2, the results presented in Chapter 5 represented a mere subset of the total flow conditions investigated. At an angle of attack of $4^{\circ}$, a total of nine Reynolds numbers were investigated, spanning from $\operatorname{Re}_{c}=70,000$ to $\operatorname{Re}_{c}=150,000$. The time-averaged surface temperature distributions from these conditions are shown in Fig. A.8a, with the corresponding distributions of streamwise gradient shown in Fig. A.8b. 


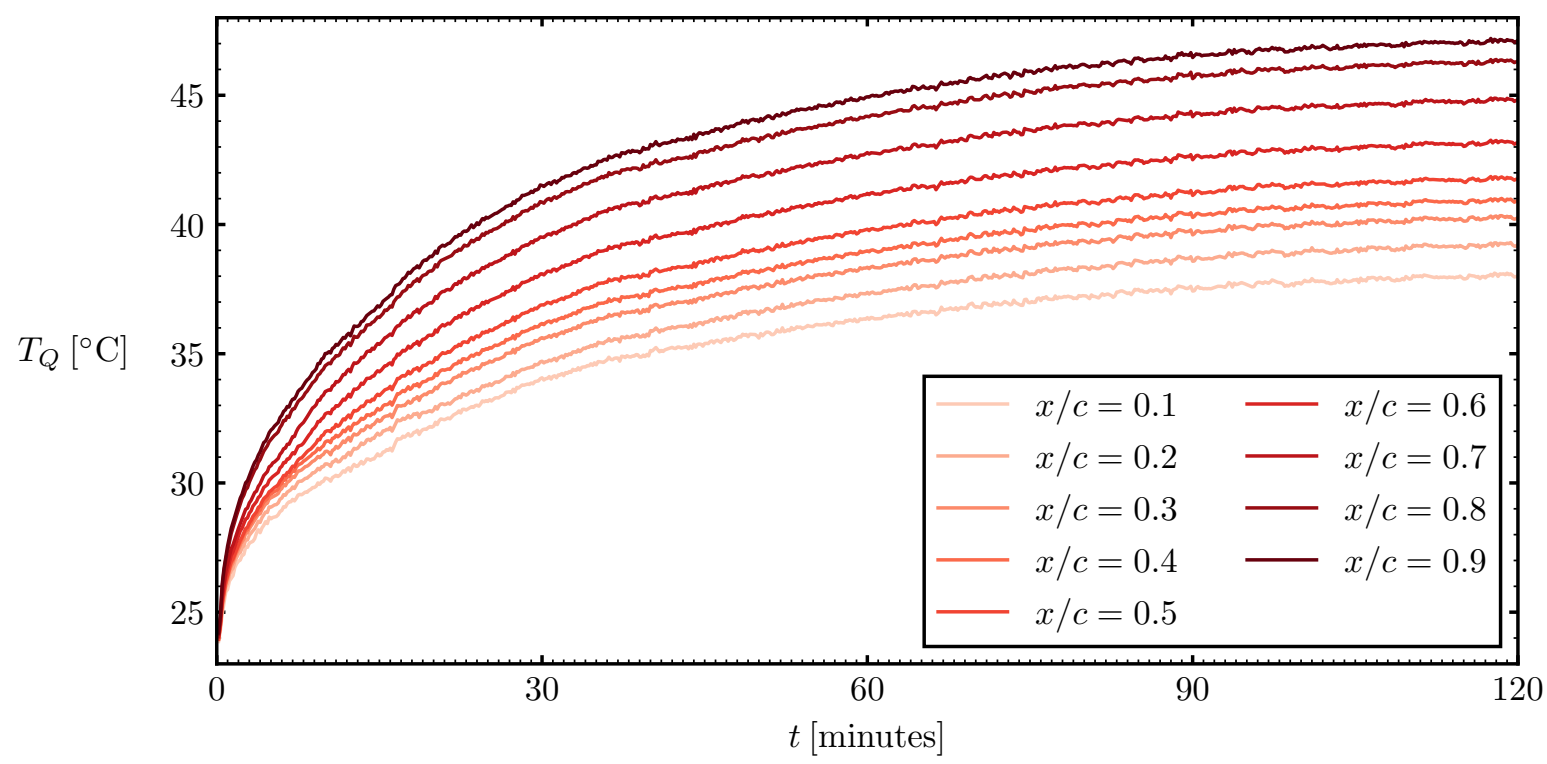

Figure A.7: Transient surface temperature development with the onset of radiative heating in quiescent conditions shown at specified streamwise locations on the airfoil.

Additionally, at the highlighted Reynolds numbers of $\operatorname{Re}_{c}=80,000$ and $\operatorname{Re}_{c}=120,000$, surface temperature distributions were measured from $\alpha=2^{\circ}$ to $\alpha=8^{\circ}$. The time-averaged distributions of surface temperature measured for these angles of attack are shown in Fig. A.9a-b, with the corresponding streamwise gradient distributions shown in Fig. A.9cd. Distributions of $h^{*}$, and corresponding streamwise gradient, were also computed at each of the flow conditions. The distributions of $h^{*}$ at $\alpha=4^{\circ}$ and each investigated Reynolds number are included in Fig. A.10a, while the corresponding streamwise gradients are shown in Fig. A.10b. Likewise, the distributions of $h^{*}$ across each investigated angle of attack at $\operatorname{Re}_{c}=80,000$ and $\mathrm{Re}_{c}=120,000$ are shown in Fig. A.11a-b, with corresponding streamwise gradients shown in Fig. A.11c-d.

Following the proposed methodology discussed in Section 5.3, the locations of mean separation, transition, and reattachment are identified at each flow condition. The estimated locations from surface temperature and $h^{*}$ distributions at $\alpha=4^{\circ}$ across all Reynolds numbers are shown in Fig. A.12, while the locations identified at highlighted Reynolds numbers of $\operatorname{Re}_{c}=80,000$ and $\operatorname{Re}_{c}=120,000$ at all investigated angles of attack are shown in Fig. A.13. The results in Figs. A.12 and A.13 show that the characteristic points estimated from surface temperature and $h^{*}$ generally agree with the expected behaviour associated with laminar separation bubbles. Although a general trend in the separation location cannot be reasonably obtained due to changes in Reynolds number, the estimated separation location displays an identifiable upstream shift as the angle of attack 


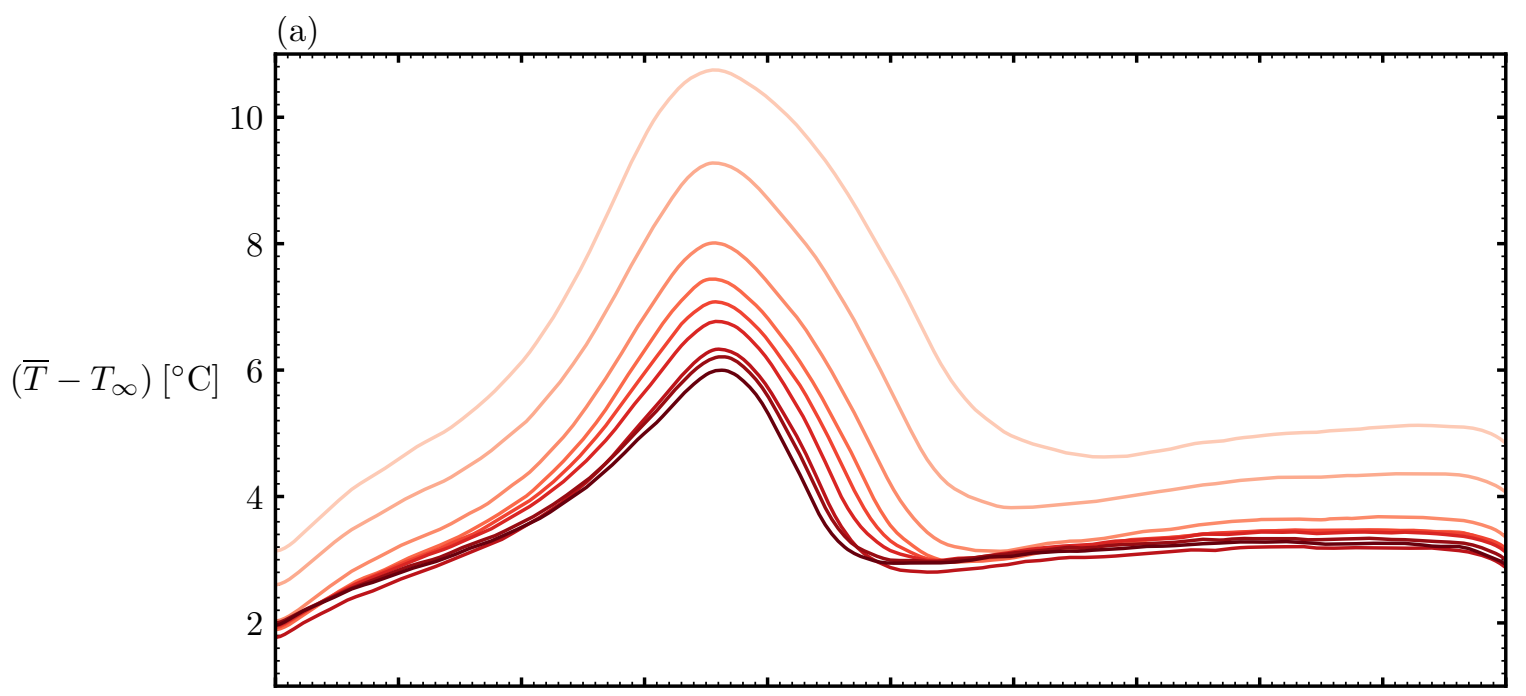

(b)

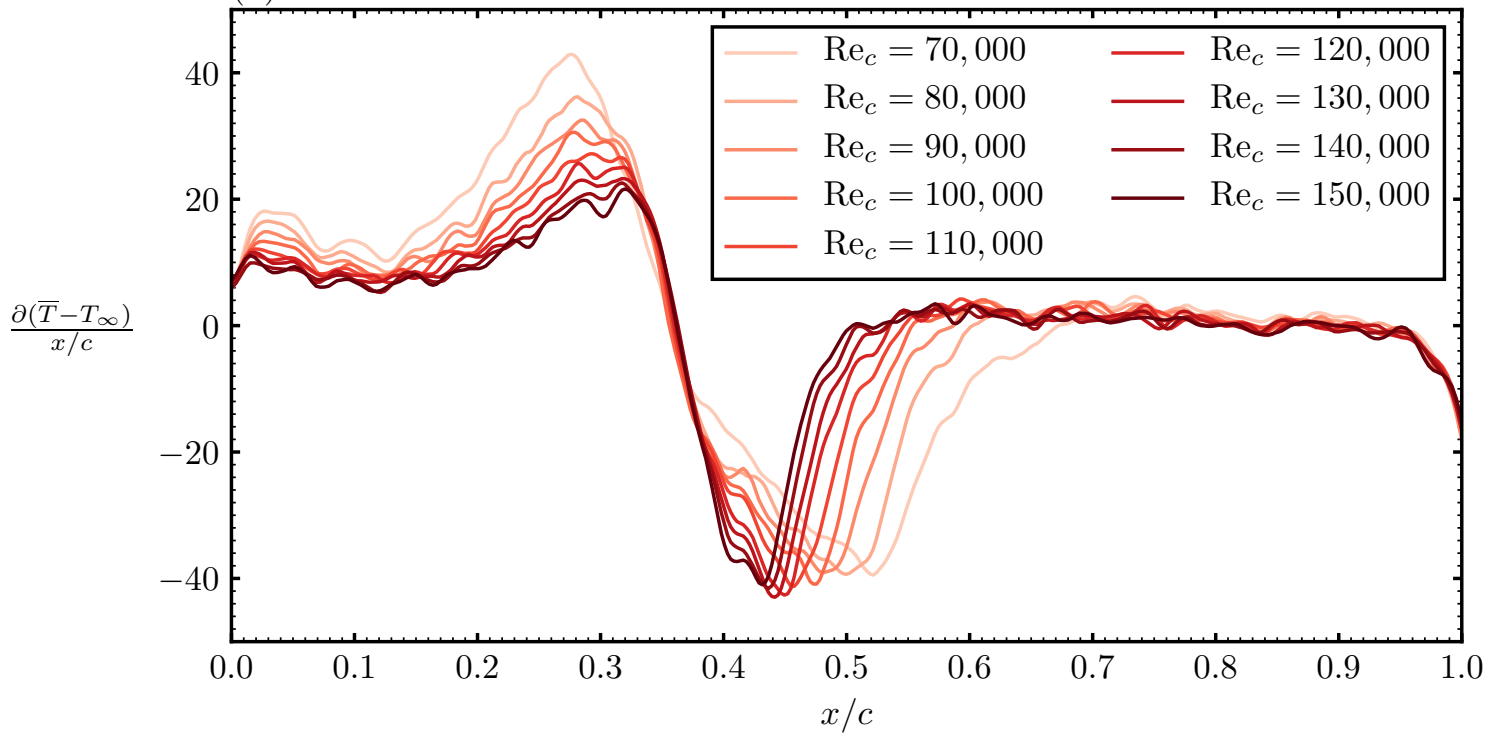

Figure A.8: Time- and spanwise-averaged distributions of (a) surface temperature, and (b) streamwise temperature gradient for each investigated Reynolds number at $\alpha=4^{\circ}$. 

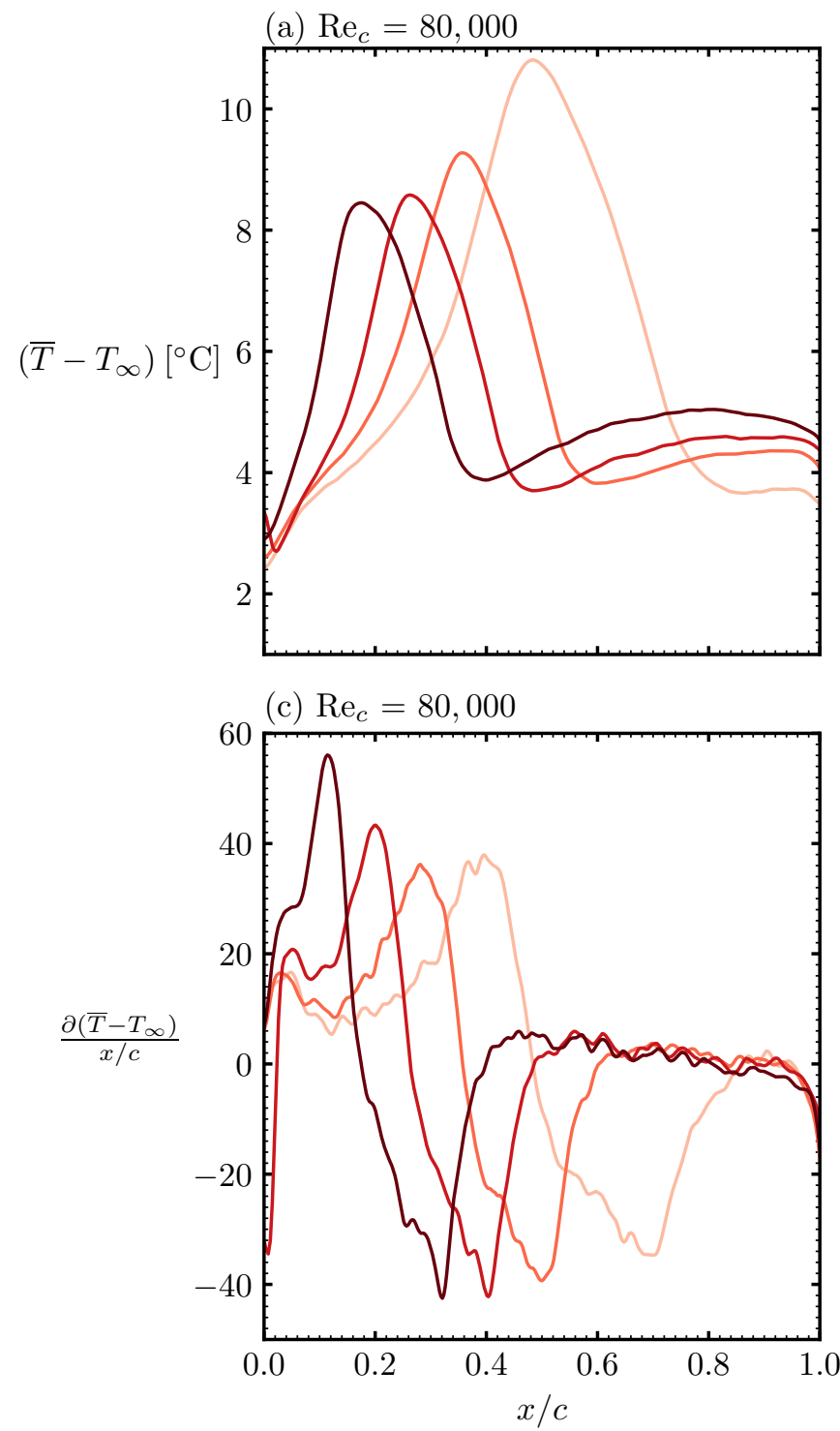

(b) $\operatorname{Re}_{c}=120,000$

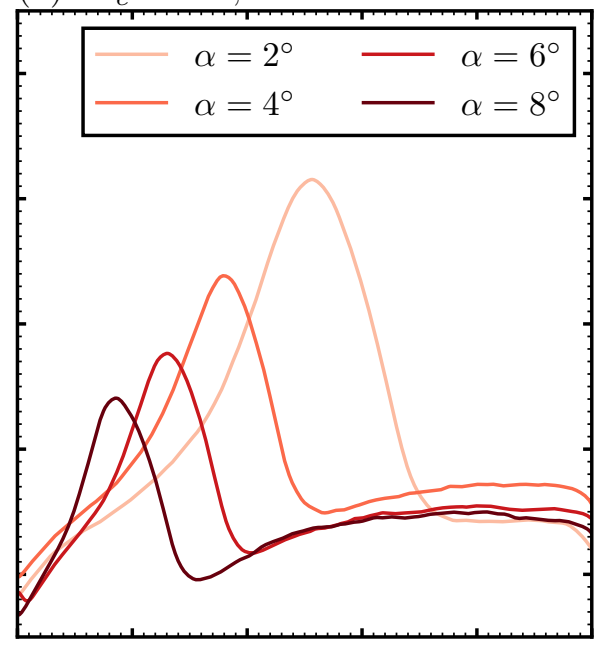

(d) $\operatorname{Re}_{c}=120,000$

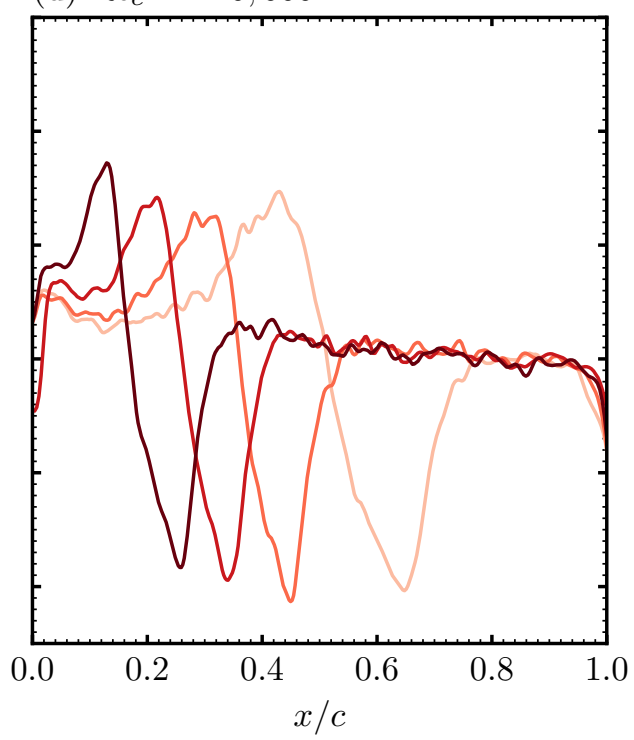

Figure A.9: Time- and spanwise-averaged distributions of (a) surface temperature at $\mathrm{Re}_{c}=80,000$, (b) surface temperature at $\mathrm{Re}_{c}=120,000$, (c) streamwise temperature gradient at $\operatorname{Re}_{c}=80,000$, and (d) streamwise temperature gradient at $\operatorname{Re}_{c}=120,000$, at each investigated angle of attack. 

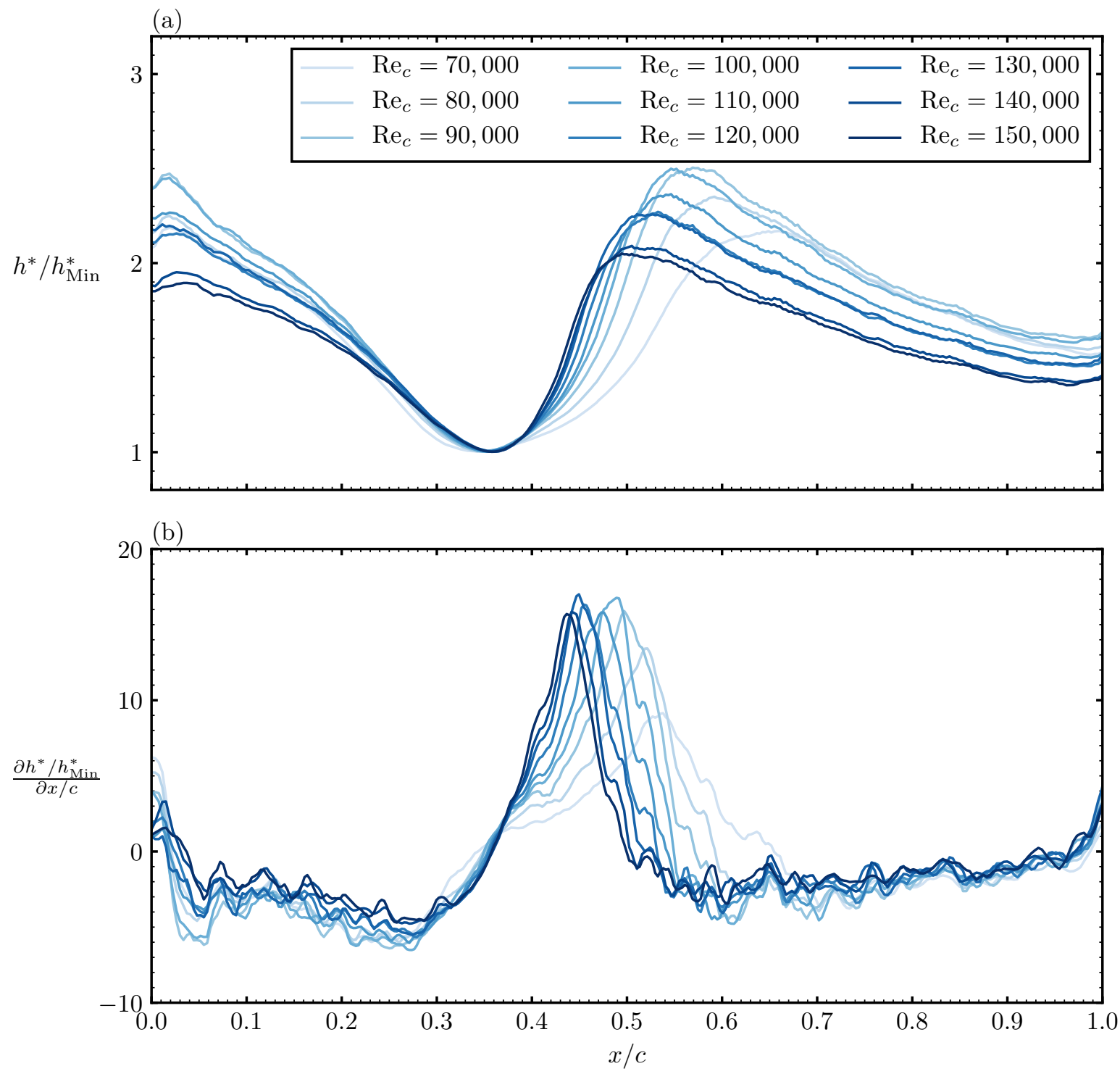

Figure A.10: Time- and spanwise-averaged distributions of (a) $h^{*}$, and (b) its streamwise gradient, for each investigated Reynolds number at $\alpha=4^{\circ}$. 


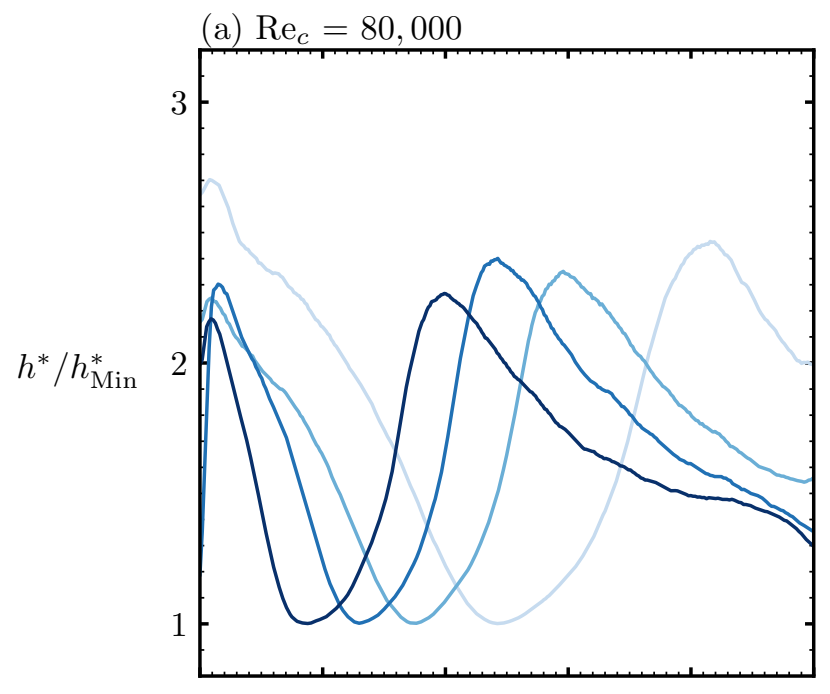

(c) $\operatorname{Re}_{c}=80,000$

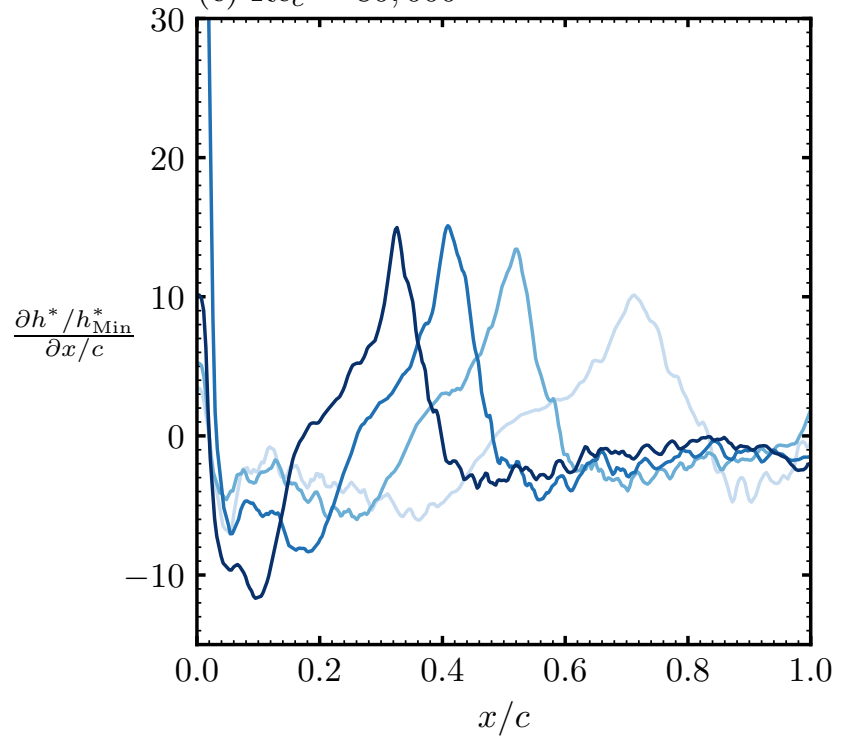

(b) $\operatorname{Re}_{c}=120,000$

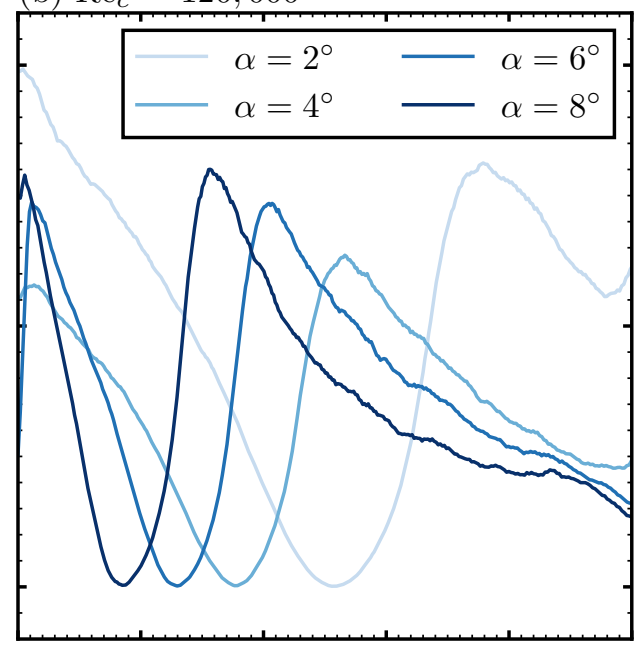

(d) $\operatorname{Re}_{c}=120,000$

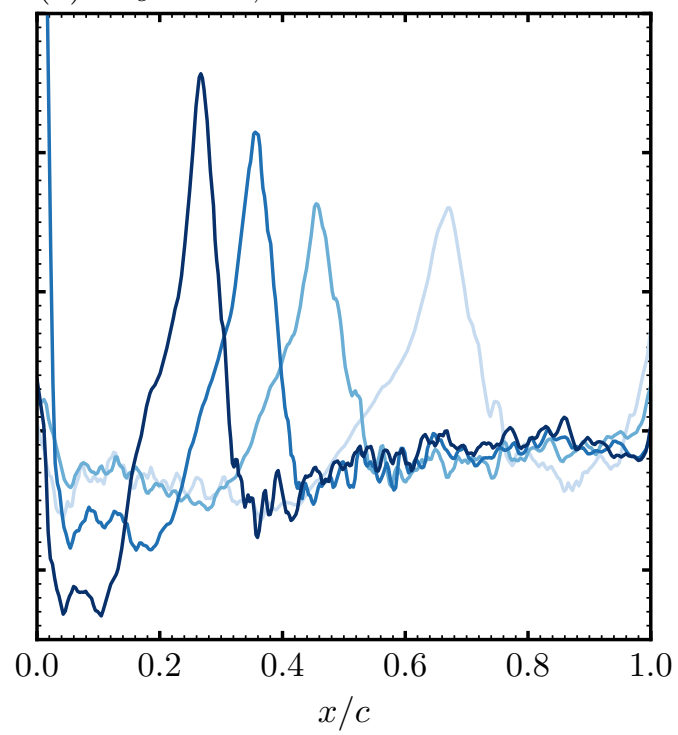

Figure A.11: Time- and spanwise-averaged distributions of (a) $h^{*}$ at $\operatorname{Re}_{c}=80,000$, (b) $h^{*}$ at $\operatorname{Re}_{c}=120,000$, (c) streamwise $h^{*}$ gradient at $\operatorname{Re}_{c}=80,000$, and (d) streamwise $h^{*}$ gradient at $\mathrm{Re}_{c}=120,000$, at each investigated angle of attack. 


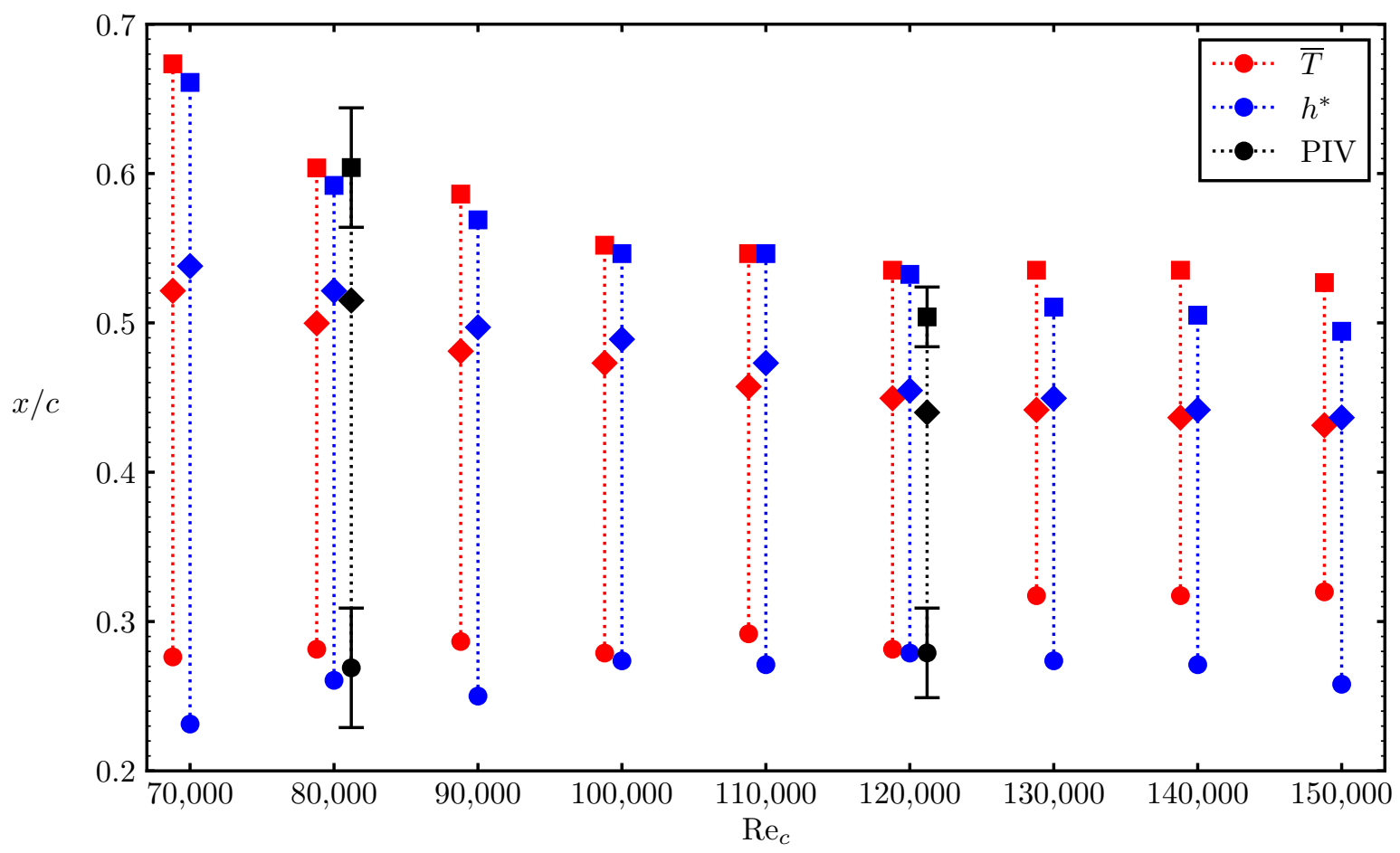

Figure A.12: Locations of mean separation, transition, and reattachment estimated from each measurement technique at $\alpha=4^{\circ}$ and each investigated Reynolds number. Mean separation, transition, and reattachment locations are indicated with circle, diamond, and square markers, respectively. A slight offset from the measurement Reynolds number is applied for visual clarity.

is increased (Fig. A.13). The locations of mean transition and reattachment are shown to advance upstream with increases in Reynolds number and angle of attack, resulting in a streamwise reduction of the bubble extent. The trends observed as the angle of attack is increased are confirmed with data from Gerakopulos et al. [8] plotted in Fig. A.13. Similar to the trends observed in the present investigation, an identifiable upstream movement of the mean separation, transition, and reattachment locations is observed as the angle of attack is increased. 


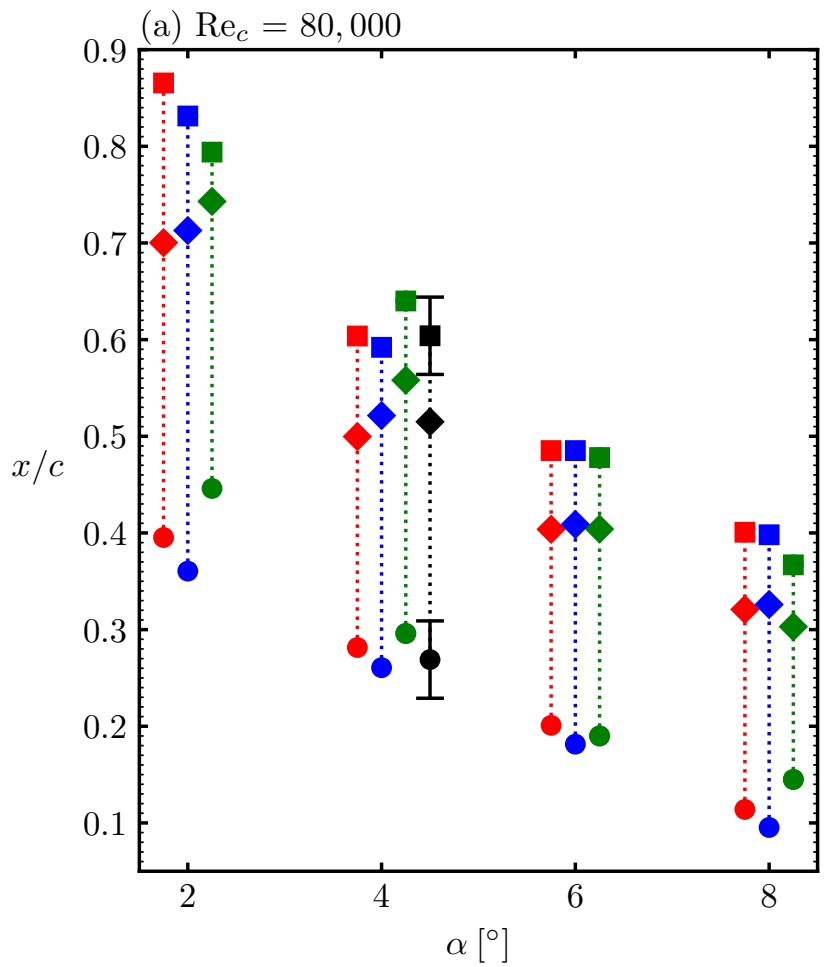

(b) $\operatorname{Re}_{c}=120,000$

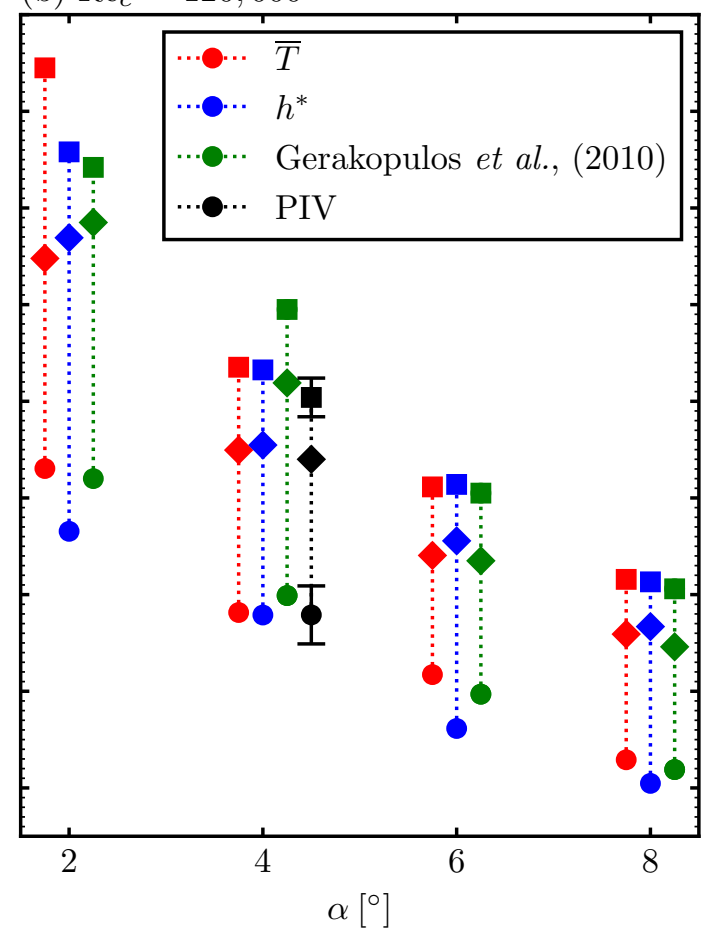

Figure A.13: Locations of mean separation, transition, and reattachment estimated from each measurement technique at (a) $\operatorname{Re}_{c}=80,000$, and (b) $\operatorname{Re}_{c}=120,000$ and each investigated angle of attack. Characteristic locations estimated from surface pressure measurements are included from Gerakopulos et al. [8]. Mean separation, transition, and reattachment locations are indicated with circle, diamond, and square markers, respectively. A slight offset from the measurement Reynolds number is applied for visual clarity. 


\section{Appendix B}

\section{Characterization of the Laminar Separation Bubble with Linear Fitting Techniques}

In order to improve the precision of estimated mean separation, transition, and reattachment locations on the flat plate geometry, linear fits were applied to distributions of surface pressure and $h^{*}$, as discussed in Chapter 4.

The methodology followed by [7] is followed in this thesis, allowing for a more precise characterization of the laminar separation bubble with surface pressure measurements. As explained in Section 4.1, the location of mean separation can be estimated at the intersection of linear lines passing through the pressure distribution upstream, and within, the pressure plateau region. The location of mean transition can be precisely identified in the pressure distribution at the onset of pressure recovery, and therefore does not require employing lines of best fit. Mean reattachment can be estimated where lines within, and downstream, of the rapid pressure recovery region intersect. This is applied to each highlighted Reynolds number in Fig. B.1, with the estimated characteristic location taken as the pressure tap nearest to the corresponding intersection point.

As shown in Section 4.2, the location of minimum streamwise gradient was not easily identifiable in the distribution of $h^{*}$, which coincides with the estimated location of mean separation. Linear lines passing through the streamwise gradient of $h^{*}$ were therefore used to estimate this location. Mean separation was estimated at the intersection of lines passing through the regions of $\frac{\partial h^{*} / h_{\text {Min }}^{*}}{\partial x / L}$ plateau, and subsequent increase. This is expected to provide a reasonable estimate for the minimum streamwise gradient location as it locates the point where convective heat transfer coefficient no longer decreases at the fastest rate. This methodology is applied at each highlighted Reynolds number, as shown in Fig. B.2. 

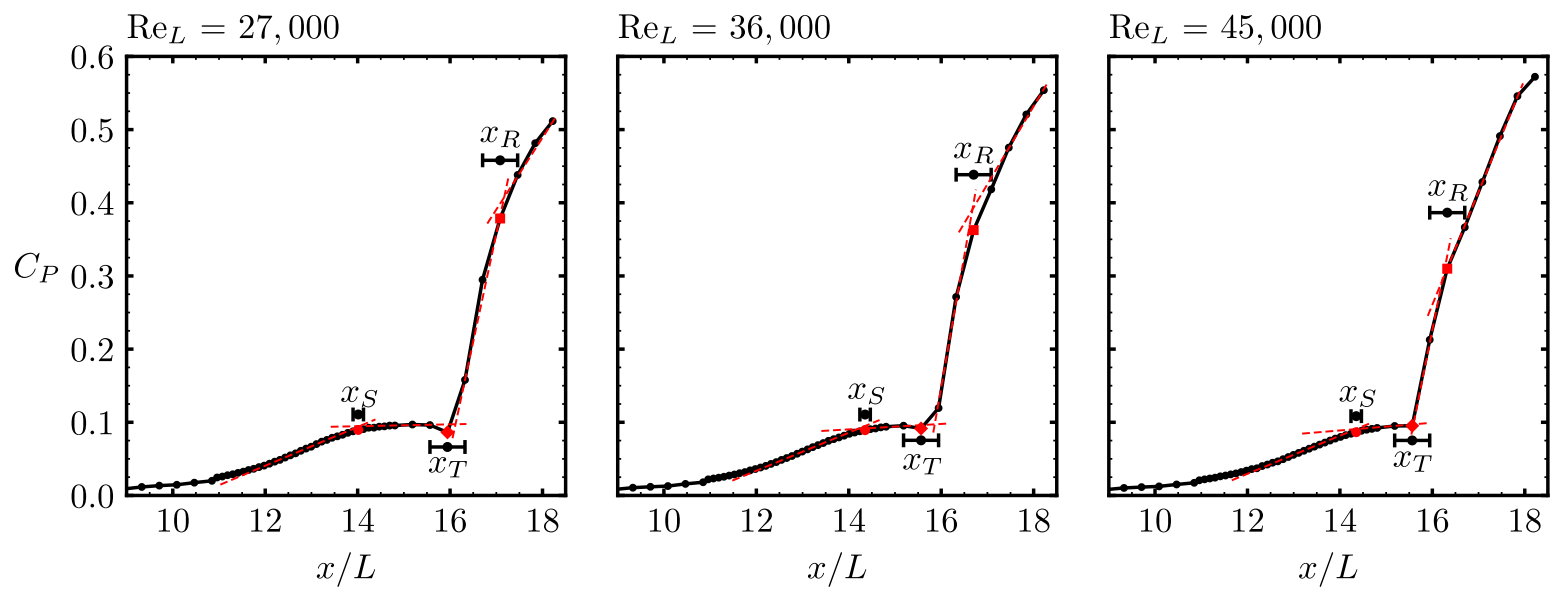

Figure B.1: Methodology employed by Boutilier \& Yarusevych [7] for estimation of the characteristic locations in a laminar separation bubble using the static pressure distribution at each highlighted Reynolds number. Uncertainty bounds are indicated with local pressure tap spacing.
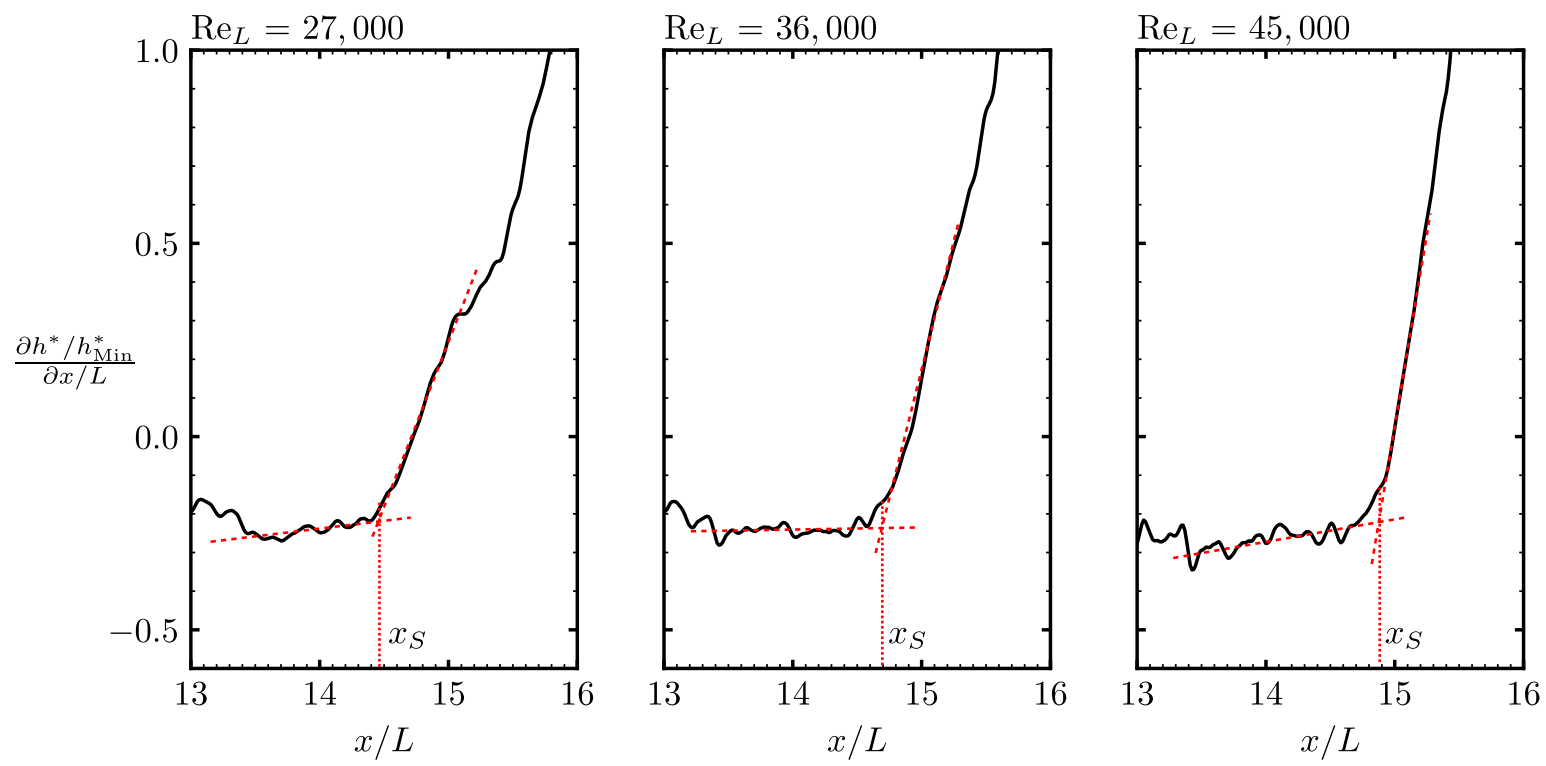

Figure B.2: Methodology used for detection of mean separation using distributions of $h^{*}$ on the flat plate at highlighted Reynolds numbers. 


\section{Appendix C}

\section{Detailed Energy Balance}

In order to perform a detailed energy balance, the heat transfer both above and within the model should be considered. In this section, the temperature distribution within the solid part of the model is computed with a numerical simulation of two-dimensional conduction. The temperature distribution within the model allows for an estimation of the wall-normal temperature gradients, which are then used in a detailed energy balance at the model surface in order to evaluate the dominant heat transfer modes, as shown for the flat plate and airfoil models in Appendices C.1 and C.2, respectively.

\section{C.1 Flat Plate Analysis}

The temperature distribution within the model is governed by the following steady-state heat conduction equation:

$$
\frac{\partial^{2} T}{\partial x^{2}}+\frac{\partial^{2} T}{\partial y^{2}}=0
$$

This governing equation is solved in the domain shown in Fig. C.1a, which represents the infrared field of view in the flat plate experiments, with the domain thickness corresponding to the thickness of the flat plate. A Dirichlet boundary condition is employed on the upper surface, where the measured surface temperature is prescribed. On the left- and right-hand boundaries, a symmetry boundary condition is applied, where the temperature is based on wall-normal heat conduction between the upper and lower surfaces. On the bottom surface, a Robin boundary condition is applied, where the conductive heat flux through the model is equated to the sum of convective and radiative heat fluxes below the model. This is shown in continuous and discrete form in Eqs. (C.2) and (C.3), respectively.

$$
k_{s} \frac{\partial T}{\partial y}=h_{B}\left(T(x, Y)-T_{\infty}\right)+\sigma \varepsilon\left(T(x, Y)^{4}-T_{\infty}^{4}\right)
$$


(a)

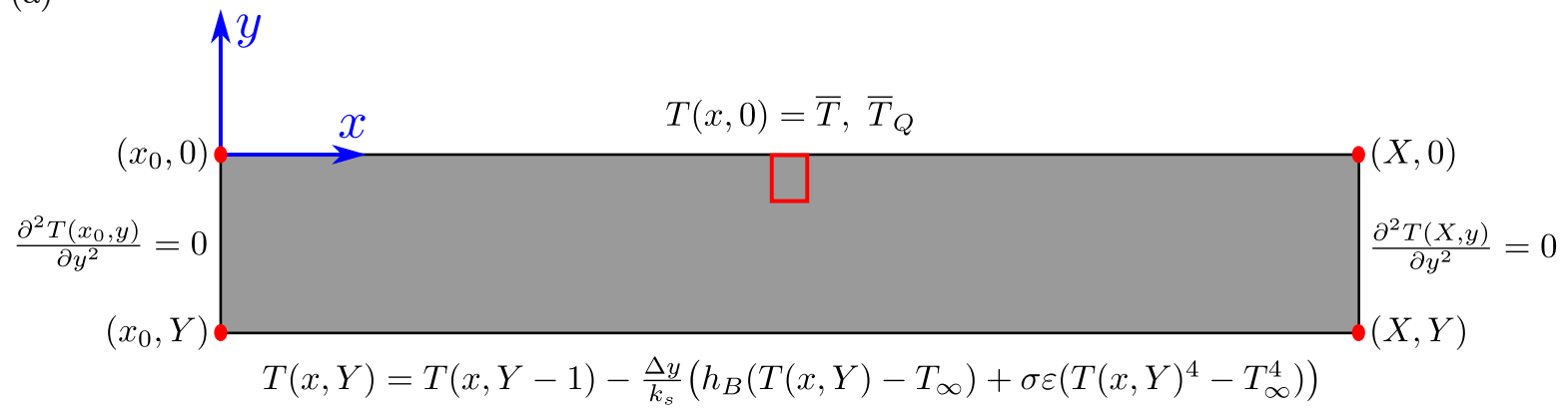

(b)

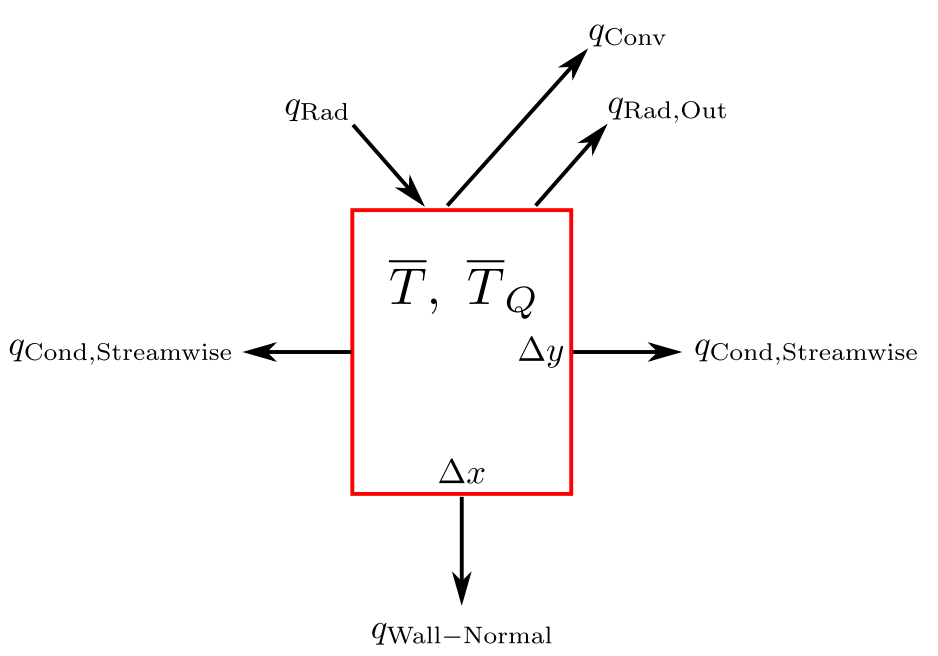

Figure C.1: Thermal diagram showing (a) the coordinate system and boundary conditions applied for the numerical thermal analysis within the flat plate model, and (b) the differential element considered in the energy balance. Note: image is not drawn to scale.

$$
T(x, Y)=T(x, Y-1)-\frac{\Delta y}{k_{s}}\left(h_{B}\left(T(x, Y)-T_{\infty}\right)+\sigma \varepsilon\left(T(x, Y)^{4}-T_{\infty}^{4}\right)\right)
$$

In Eq. C.3, $y=Y$ represents the bottom surface and $y=Y-1$ represents the temperature node directly above the bottom surface. The convective heat transfer coefficients at the bottom surface in quiescent and flow conditions, $h_{B, Q}$ and $h_{B}$, are estimated to be 1.5 and $25 \mathrm{~W} \mathrm{~m}^{-2} \mathrm{~K}^{-1}$, respectively. The quiescent convective heat transfer coefficient is estimated based on a flat plate free convection correlation (Eqn. 9.32 in [122]), whereas the forced convective heat transfer coefficient is estimated from the turbulent boundary layer correlation shown in Eq. 2.8.

With the boundary conditions described above, Eq. C.1 is discretized using second order central differences within the domain core, and the resulting system of algebraic equations is then solved numerically using a Jacobi iterative numerical solver. The results obtained 

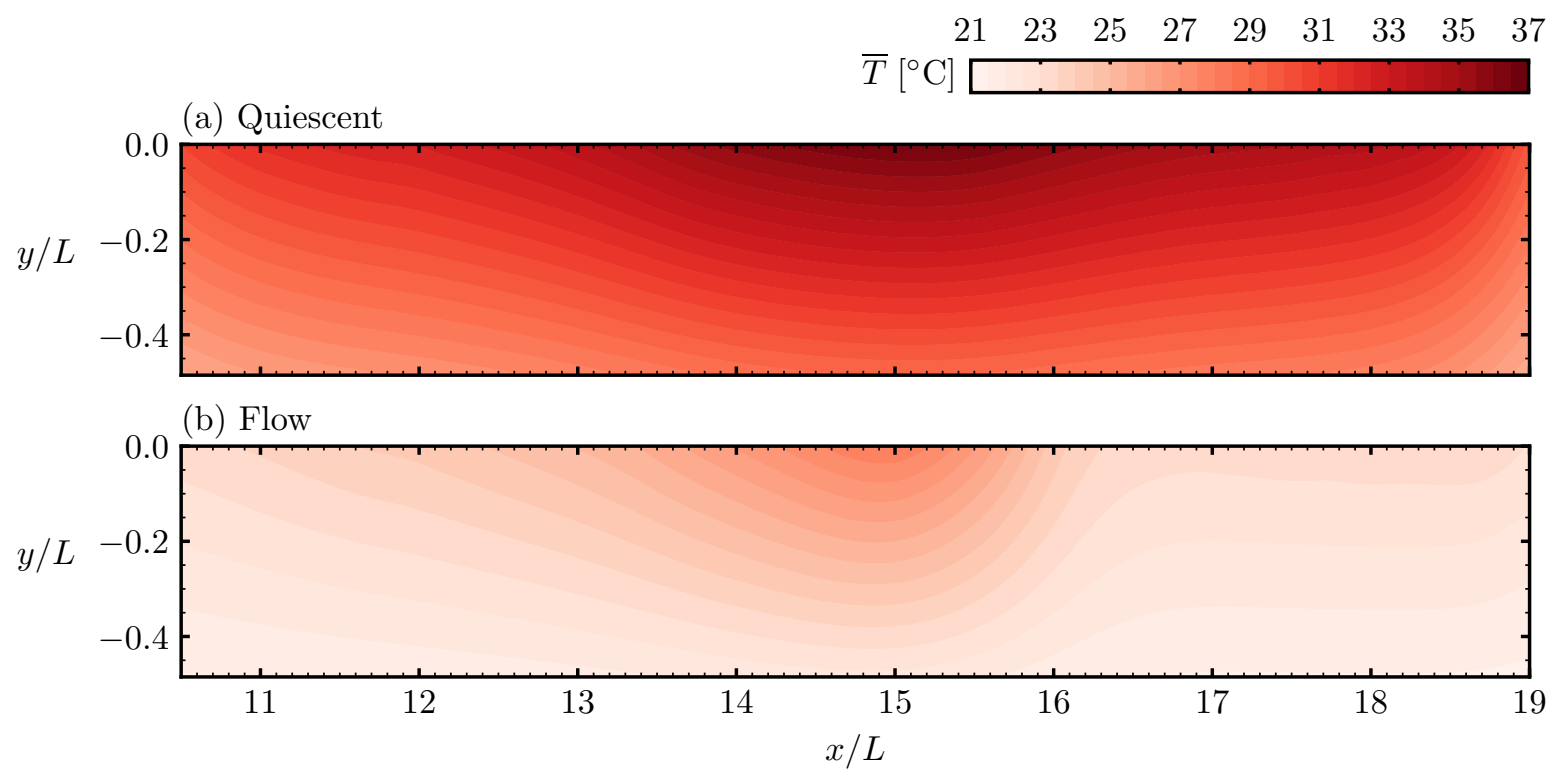

Figure C.2: Time-averaged temperature distribution within the flat plate model in (a) quiescent, and (b) flow conditions $\left(\operatorname{Re}_{L}=27,000\right)$ determined with numerical analysis.

for the quiescent and flow conditions are shown in Fig. C.2. While the employed boundary conditions at the left- and right-hand boundaries are a relatively course approximation, the results are expected to provide a reasonable approximation of the temperature distribution within the domain core, under the laminar separation bubble. Thus, using the temperature distributions shown in Fig. C.2, a quantitative evaluation of the dominant heat transfer modes can be performed.

Considering a differential element on the flat plate surface (Fig. C.1b), the energy balance in thermal equilibrium, considering streamwise conduction, free convection, wallnormal heat flux (including conduction through the model, convection at the bottom surface, and radiative cooling at the bottom surface), and radiative heating and cooling, can be written as:

$$
q_{\text {Rad }}+q_{\text {Cond,Streamwise }}+q_{\text {Wall-Normal }}+q_{\text {Conv }}+q_{\text {Rad,Out }}=0
$$

Assuming the incident radiative heat flux to be positive, and the convective, conductive, wall-normal, and radiative cooling heat fluxes to be negative, as shown in Fig. C.1b, Eq. C.4 can be expanded and rearranged for quiescent conditions, yielding:

$$
\frac{q_{\text {Rad }}}{\Delta z}=k_{s} \Delta y \frac{\partial \bar{T}_{Q}}{\partial x}+k_{s} \Delta x \frac{\partial \bar{T}_{Q}}{\partial y}+h_{\text {Free }} \Delta x\left(\bar{T}_{Q}-T_{\infty}\right)+\varepsilon \sigma \Delta x\left(\bar{T}_{Q}^{4}-T_{\infty}^{4}\right)
$$


where the derivative terms are obtained from the solution of the numerical analysis (Fig. C.2).

A similar analysis can be performed on in-flow temperature measurements, where the differential energy balance can be written as:

$$
\frac{q_{\mathrm{Rad}}}{\Delta z}=k_{s} \Delta y \frac{\partial \bar{T}}{\partial x}+k_{s} \Delta x \frac{\partial \bar{T}}{\partial y}+h \Delta x\left(\bar{T}-T_{\infty}\right)+\varepsilon \sigma \Delta x\left(\bar{T}^{4}-T_{\infty}^{4}\right)
$$

Equating Eqs. (C.5) and (C.6), and simplifying the expression yields:

$$
\begin{aligned}
& k_{s} \Delta y \frac{\partial \bar{T}}{\partial x}+k_{s} \Delta x \frac{\partial \bar{T}}{\partial y}+h \Delta x\left(\bar{T}-T_{\infty}\right)+\varepsilon \sigma \Delta x\left(\bar{T}^{4}-T_{\infty}^{4}\right)= \\
& k_{s} \Delta y \frac{\partial \bar{T}_{Q}}{\partial x}+k_{s} \Delta x \frac{\partial \bar{T}_{Q}}{\partial y}+h_{\mathrm{Free}} \Delta x\left(\bar{T}_{Q}-T_{\infty}\right)+\varepsilon \sigma \Delta x\left(\bar{T}_{Q}^{4}-T_{\infty}^{4}\right)
\end{aligned}
$$

Solving for the convective heat transfer coefficient, $h$, and simplifying the expression yields:

$$
h=\frac{k_{s} \Delta y\left(\frac{\partial \bar{T}_{Q}}{\partial x}-\frac{\partial \bar{T}}{\partial x}\right)}{\Delta x\left(\bar{T}-T_{\infty}\right)}+\frac{k_{s}\left(\frac{\partial \bar{T}_{Q}}{\partial y}-\frac{\partial \bar{T}}{\partial y}\right)}{\left(\bar{T}-T_{\infty}\right)}+h_{\text {Free }}\left(\frac{\bar{T}_{Q}-T_{\infty}}{\bar{T}-T_{\infty}}\right)+\varepsilon \sigma\left(\frac{\bar{T}_{Q}^{4}-\bar{T}^{4}}{\bar{T}-T_{\infty}}\right)
$$

Table C.1: Summary of variables, and their respective values, used in the full energy balance on the flat plate model.

\begin{tabular}{ccc}
\hline \hline Variable & Description & Value \\
\hline$\Delta x$ & differential streamwise coordinate & $0.0007 \mathrm{~m}$ \\
$\Delta y$ & differential wall-normal coordinate & $0.0007 \mathrm{~m}$ \\
$h_{\text {Free }}$ & free convective heat transfer coefficient & $3 \mathrm{Wm}^{-2} \mathrm{~K}^{-1 *}$ \\
$k_{s}$ & thermal conductivity of the plate & $0.20 \mathrm{Wm}^{-1} \mathrm{~K}^{-1}$ \\
$\varepsilon$ & surface emissivity & $0.95^{*}$ \\
$\sigma$ & Stefan-Boltzmann constant & $5.67 \times 10^{-8} \mathrm{Wm}^{-2} \mathrm{~K}^{-4}$ \\
$\bar{T}$ & mean in-flow temperature & measured \\
$\bar{T}_{Q}$ & mean quiescent temperature & measured \\
$T_{\infty}$ & ambient temperature & $21^{\circ} \mathrm{C}$ \\
\hline \hline
\end{tabular}

* Assumed value

In the expression shown in Eq. C.8, the first term is related to the difference in streamwise conduction between quiescent and flow conditions, the second term is related to the difference in wall-normal heat flux between quiescent and flow conditions, the third term is related to free convection, and the fourth term is related to the difference in radiative cooling between quiescent and flow conditions. With such an arrangement, the rela- 


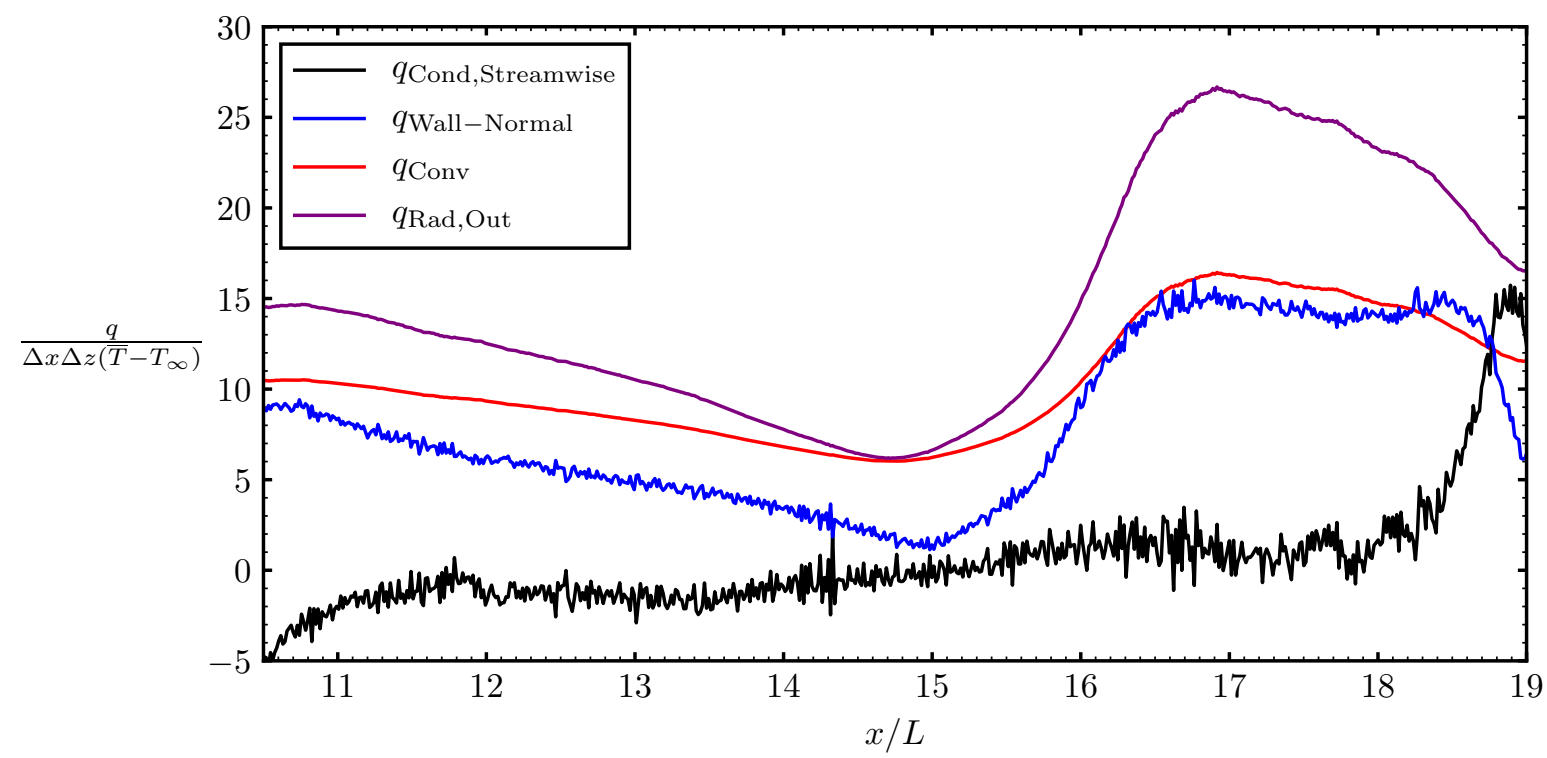

Figure C.3: Contribution of each heat transfer mode to the variation of convective heat transfer coefficient in the flat plate energy balance. Data pertains to $\operatorname{Re}_{L}=27,000$.

tive contribution to the variation of convective heat transfer coefficient can be quantified. Equation C.8 is computed at each streamwise location with the various parameters summarized in Table C.1. The results presented in Fig. C.3 highlight the relative contribution of each heat flux term to the overall convective heat transfer coefficient. It can be seen from Fig. C.3 that the component of Eq. C.8 contributing to streamwise conduction is of negligible magnitude, and simply contributes to the spatial fluctuations. Additionally, Fig. C.3 shows that the wall-normal heat flux, the heat flux associated with convection, and the radiative cooling heat flux exhibit a similar streamwise variation with comparable magnitudes. The resulting variation of convective heat transfer coefficient is shown in Fig. C.4, highlighting the difference in convective heat transfer coefficient distribution when secondary modes of heat transfer are considered. The results show that the magnitude of convective heat transfer coefficient and its streamwise gradient are affected when secondary modes are considered; however, the extrema locations do not change appreciably. Additionally, the variation of convective heat transfer coefficient and its streamwise gradient exhibit increased spatial fluctuations when secondary heat transfer modes are considered. It can therefore be concluded that the characteristic locations of the laminar separation bubble can be reasonably estimated by considering only the convective heat transfer mode. 


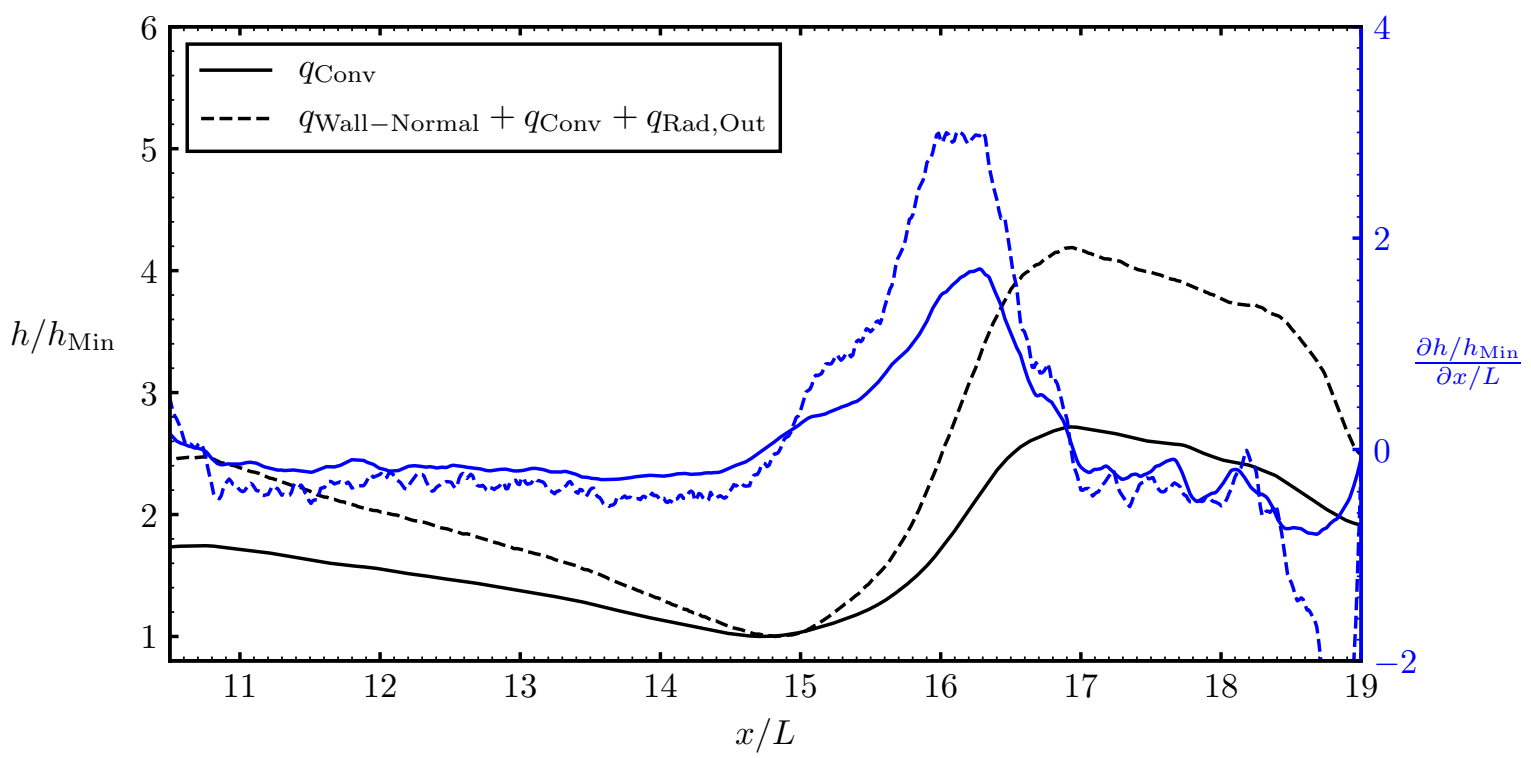

Figure C.4: Normalized distributions of convective heat transfer coefficient where the effect of considering radiative cooling and wall-normal heat flux is assessed. Data are presented with corresponding streamwise gradients at $\operatorname{Re}_{L}=27,000$.

\section{C.2 Airfoil Energy Balance}

Similar to the flat plate analysis, the temperature distribution within the $5 \mathrm{~mm}$ airfoil shell is governed by two-dimensional heat conduction (Eq. C.1). Equation C.1 is solved in the domain shown in Fig. C.5a with the associated boundary conditions. On the upper surface, a Dirichlet boundary condition is employed, where the time- and spanwise-averaged surface temperature is prescribed. Due to the thermal insulation provided by the air-filled airfoil core, a Neumann boundary condition is prescribed at the bottom surface of the airfoil shell, such that the wall-normal heat flux is equal to zero. A symmetry boundary condition is applied to the left- and right-hand boundaries, where the temperature is governed by the wall-normal conductive heat flux. Due to the zero-flux boundary condition at the bottom surface, and the prescribed temperature at the top surface, the symmetry boundary condition on the left- and right-hand boundaries results in a constant temperature along the side edges of the domain. Although this is a course approximation, it is assumed that the area of interest (i.e. beneath the laminar separation bubble) is sufficiently far from the boundaries such that the temperature in this region is not sensitive to minute changes in the boundary condition.

Equation C.1 is discretized using second order central difference within the domain shown in Fig. C.5a, and the resulting system of algebraic equations is then solved with a Jacobi iterative numerical solver. The resulting temperature within the airfoil shell is 
(a)

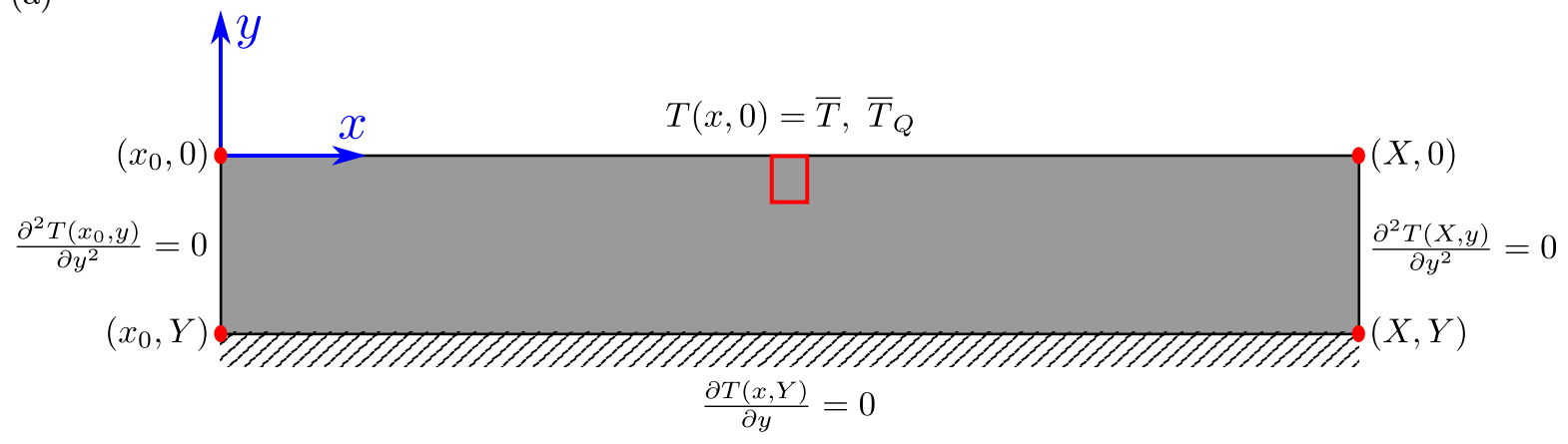

(b)

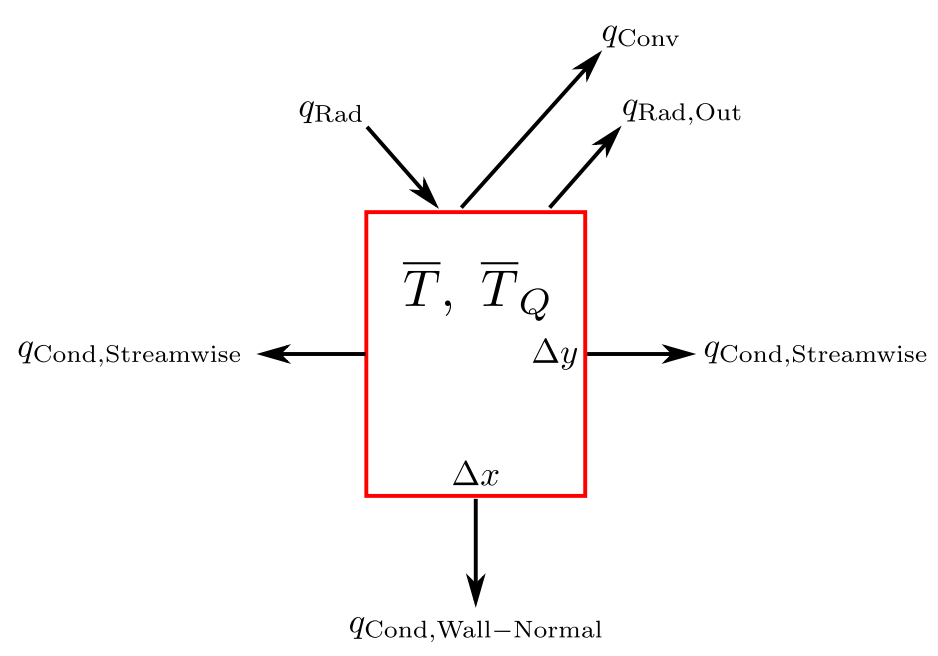

Figure C.5: Thermal diagram showing (a) the coordinate system and boundary conditions applied for the numerical thermal analysis within the airfoil model, and (b) the differential element considered in the energy balance. Note: image is not drawn to scale.

shown for quiescent and flow conditions in Fig. C.6. Here, the surface curvature, specifically where laminar separation bubbles are observed, is assumed to be sufficiently small such that the airfoil shell can be modelled in orthogonal cartesian coordinates. Additionally, it is assumed that the boundary conditions applied provide a reasonable approximation of the physical boundary conditions, such that the airfoil core temperature distribution in the streamwise region associated with laminar separation bubbles is sufficiently accurate, allowing for a quantitative evaluation of the dominant heat transfer modes.

Following the same approach as the one established in Appendix C.1, an energy balance on a differential element at the airfoil surface (Fig. C.5a) can be performed. In thermal equilibrium, an energy balance for the differential element can be written as:

$$
q_{\text {Rad }}+q_{\text {Cond,Streamwise }}+q_{\text {Cond,Wall-Normal }}+q_{\text {Conv }}+q_{\text {Rad,Out }}=0
$$



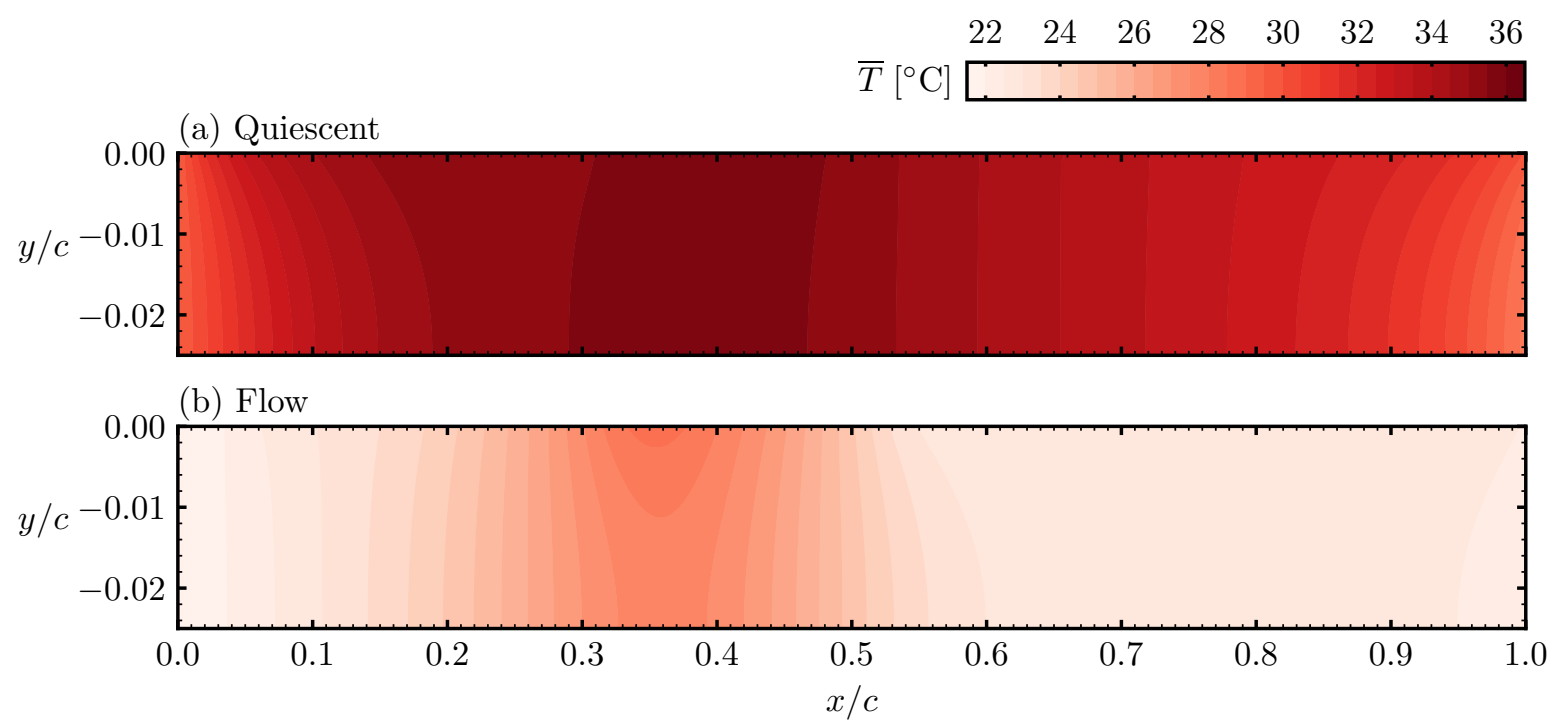

Figure C.6: Time-averaged temperature distribution within the airfoil model in (a) quiescent, and (b) flow conditions $\left(\operatorname{Re}_{c}=80,000\right)$ determined with numerical analysis.

Assuming the incident radiative heat flux to be positive, and the convective, conductive, and outgoing radiative heat fluxes to be negative, as shown in Fig. C.5b, Eq. C.9 can be expanded and rearranged for quiescent conditions, yielding:

$$
\frac{q_{\mathrm{Rad}}}{\Delta z}=k_{s} \Delta y \frac{\partial \bar{T}_{Q}}{\partial x}+k_{s} \Delta x \frac{\partial \bar{T}_{Q}}{\partial y}+h_{\mathrm{Free}} \Delta x\left(\bar{T}_{Q}-T_{\infty}\right)+\varepsilon \sigma \Delta x\left(\bar{T}_{Q}^{4}-T_{\infty}^{4}\right)
$$

A similar analysis can be performed for in-flow temperature measurements, yielding:

$$
\frac{q_{\mathrm{Rad}}}{\Delta z}=k_{s} \Delta y \frac{\partial \bar{T}}{\partial x}+k_{s} \Delta x \frac{\partial \bar{T}}{\partial y}+h \Delta x\left(\bar{T}-T_{\infty}\right)+\varepsilon \sigma \Delta x\left(\bar{T}^{4}-T_{\infty}^{4}\right)
$$

Equating Eqs. (C.10) and (C.11), and simplifying the expression yields:

$$
\begin{aligned}
& k_{s} \Delta y \frac{\partial \bar{T}}{\partial x}+k_{s} \Delta x \frac{\partial \bar{T}}{\partial y}+h \Delta x\left(\bar{T}-T_{\infty}\right)+\varepsilon \sigma \Delta x\left(\bar{T}^{4}-T_{\infty}^{4}\right)= \\
& k_{s} \Delta y \frac{\partial \bar{T}_{Q}}{\partial x}+k_{s} \Delta x \frac{\partial \bar{T}_{Q}}{\partial y}+h_{\text {Free }} \Delta x\left(\bar{T}_{Q}-T_{\infty}\right)+\varepsilon \sigma \Delta x\left(\bar{T}_{Q}^{4}-T_{\infty}^{4}\right)
\end{aligned}
$$

which can be solved for the convective heat transfer coefficient, $h$, and simplified, yielding:

$$
h=\frac{k_{s} \Delta y\left(\frac{\partial \bar{T}_{Q}}{\partial x}-\frac{\partial \bar{T}}{\partial x}\right)}{\Delta x\left(\bar{T}-T_{\infty}\right)}+\frac{k_{s}\left(\frac{\partial \bar{T}_{Q}}{\partial y}-\frac{\partial \bar{T}}{\partial y}\right)}{\left(\bar{T}-T_{\infty}\right)}+h_{\text {Free }}\left(\frac{\bar{T}_{Q}-T_{\infty}}{\bar{T}-T_{\infty}}\right)+\varepsilon \sigma\left(\frac{\bar{T}_{Q}^{4}-\bar{T}^{4}}{\bar{T}-T_{\infty}}\right)
$$


Table C.2: Summary of variables, and their respective values, used in the full energy balance on the airfoil model.

\begin{tabular}{ccc}
\hline \hline Variable & Description & Value \\
\hline$\Delta x$ & differential streamwise coordinate & $0.0006 \mathrm{~m}$ \\
$\Delta y$ & differential wall-normal coordinate & $0.0006 \mathrm{~m}$ \\
$h_{\text {Free }}$ & free convective heat transfer coefficient & $5 \mathrm{Wm}^{-2} \mathrm{~K}^{-1}$ \\
$k_{s}$ & thermal conductivity of the airfoil shell & $0.20 \mathrm{Wm}^{-1} \mathrm{~K}^{-1}$ \\
$\varepsilon$ & surface emissivity & $0.95^{*}$ \\
$\sigma$ & Stefan-Boltzmann constant & $5.67 \times 10^{-8} \mathrm{Wm}^{-2} \mathrm{~K}^{-4}$ \\
$\bar{T}$ & mean in-flow temperature & measured \\
$\bar{T}_{Q}$ & mean quiescent temperature & measured \\
$T_{\infty}$ & ambient temperature & $19.5{ }^{\circ} \mathrm{C}$ \\
\hline \hline
\end{tabular}

${ }^{*}$ Assumed value

Equation C.13 is computed at each streamwise location with the various parameters summarized in Table C.2. The results presented in Fig. C.7 show the relative contributions of each heat transfer mode to the variation of convective heat transfer coefficient. Again, it is shown that the streamwise conduction does not contribute significantly and can be reasonably neglected. The convective, radiative, and wall-normal heat flux terms all exhibit a similar streamwise variation with comparable magnitudes. The effect of considering such secondary heat transfer modes in regards to the variation of convective heat transfer coefficient is shown in Fig. C.8. Similar to the flat plate experiments, it is shown that the streamwise variation of convective heat transfer coefficient is altered in terms of magnitude when the secondary heat transfer modes are considered; however, the extrema locations remain relatively unchanged within the experimental uncertainty limits. Therefore, these secondary heat transfer modes can be reasonably neglected for the characterization of laminar separation bubbles. 


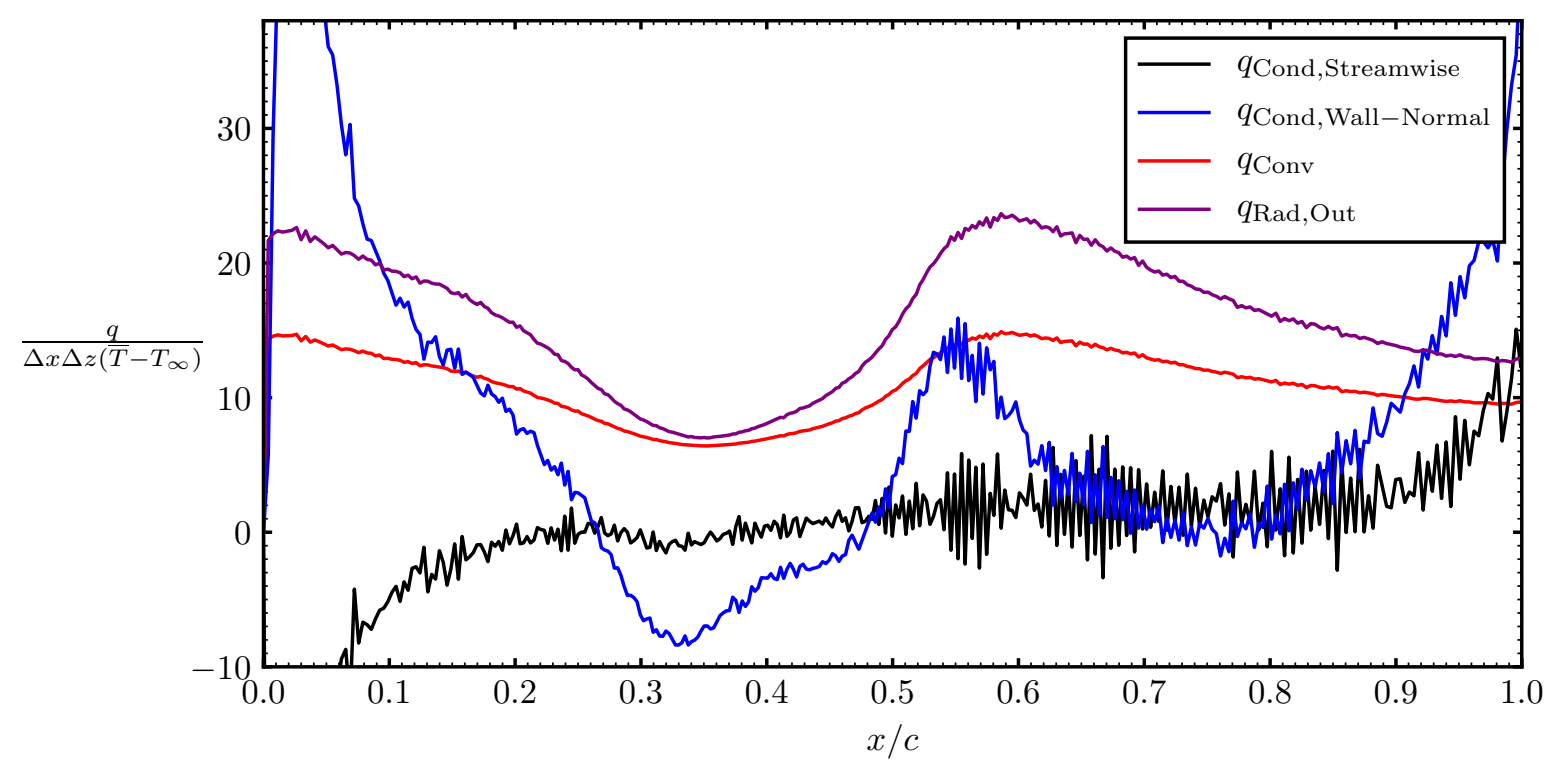

Figure C.7: Contribution of each heat transfer mode to the variation of convective heat transfer coefficient in the airfoil energy balance. Data pertains to $\operatorname{Re}_{c}=80,000$ and $\alpha=4^{\circ}$.

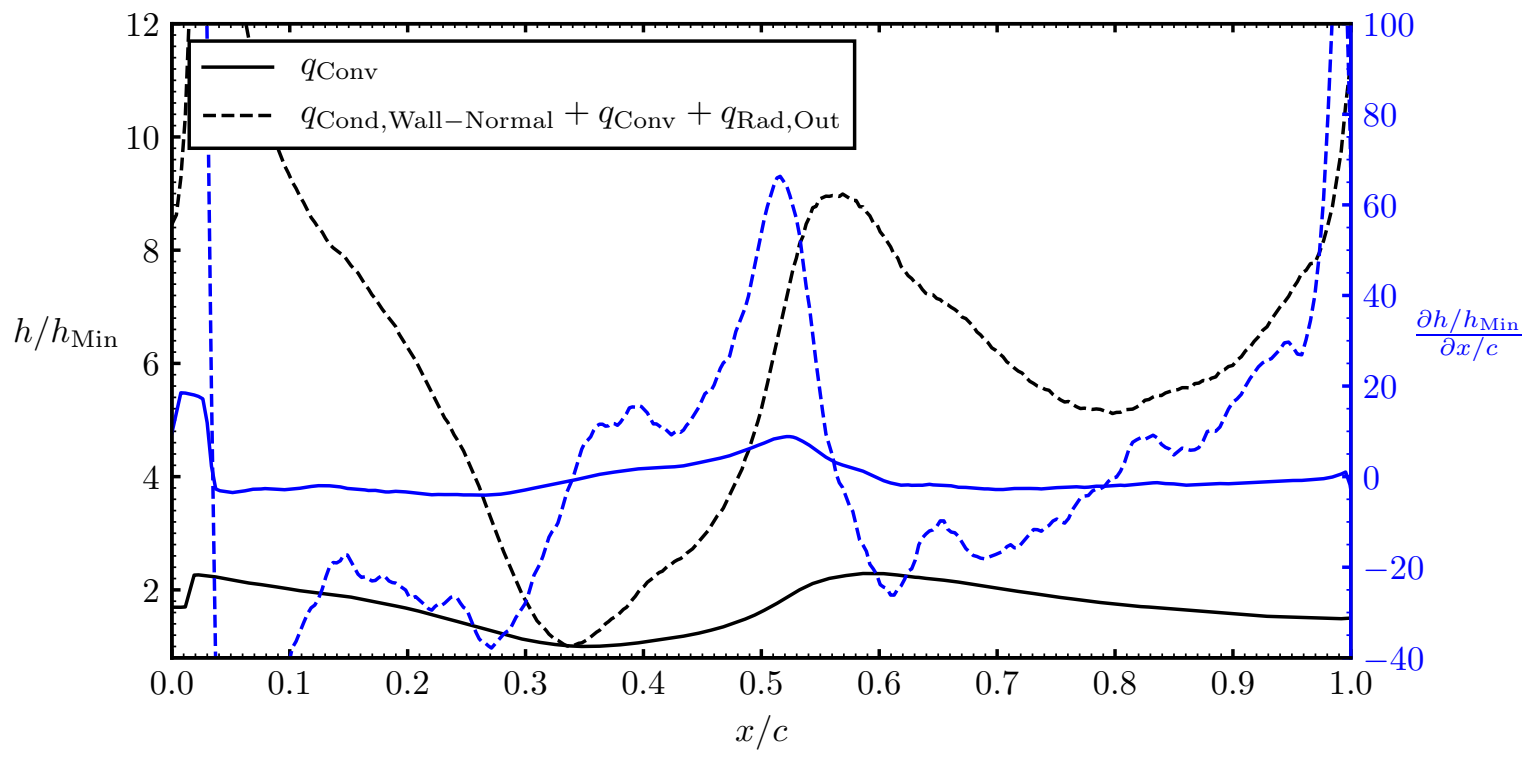

Figure C.8: Normalized distributions of convective heat transfer coefficient where the effect of considering radiative cooling and wall-normal conduction is assessed. Data are presented with corresponding streamwise gradients at $\operatorname{Re}_{c}=80,000$ and $\alpha=4^{\circ}$. 


\section{Appendix D}

\section{Experimental Uncertainty}

As the objective of this thesis was to provide a comparative analysis of laminar separation bubble characterization between several measurement techniques, it is important to quantify the uncertainty associated with these techniques. The uncertainty estimates presented are quantified using the $n^{\text {th }}$ order approach, described in detail by Moffat [123-125], which considers significant uncertainty contributions from $n$ sources. For a particular quantity, $R$, the total uncertainty, $\psi_{R}$, is given by the root-sum-square of the individual uncertainty contributions:

$$
\psi_{R}= \pm \sqrt{\sum_{i=1}^{n} \psi_{i}^{2}}
$$

For the cases where uncertainty cannot be directly determined, uncertainty propagation must be implemented. For a particular quantity, $R$, which is calculated based on a known function, the total uncertainty can be calculated:

$$
\psi_{R}= \pm \sqrt{\sum_{i=1}^{n}\left(\frac{\partial R}{\partial \epsilon_{i}} \psi_{\epsilon_{i}}\right)^{2}}
$$

where $\epsilon_{i}$ is a measured variable which is used in the calculation of $R$, associated with an uncertainty $\psi_{\epsilon_{i}}$. The uncertainty values calculated in this chapter are summarized in Tables D.1 and D.2 for experiments on the flat plate and airfoil geometries, respectively.

\section{D.1 Uncertainty in Experimental Conditions}

The uncertainty associated with free-stream Reynolds number depends on uncertainty in the characteristic length, kinematic viscosity, and free-stream velocity. The characteristic length can be measured to an accuracy of $\pm 0.5 \mathrm{~mm}$, or $\pm 1 \%$ and $\pm 0.5 \%$ of the character- 
Table D.1: Summary of uncertainty estimates for flat plate experiments.

\begin{tabular}{|c|c|c|c|}
\hline Quantity & Conditions & Uncertainty $^{*}$ & Applies to \\
\hline $\operatorname{Re}_{L}$ & & $\pm 2 \%$ & \\
\hline$C_{P}$ & & {$[ \pm 0.03, \pm 0.02, \pm 0.02]$} & Figs. 4.1-4.3, A.1, and B.1 \\
\hline$T$ & & $\pm 0.04^{\circ} \mathrm{C}$ & Figs. $4.10-4.12$, A.3, and A.4 \\
\hline$h^{*}$ & & {$[ \pm 0.006, \pm 0.007, \pm 0.01]$} & Figs. 4.13 and A.6 \\
\hline$x_{S}$ & Pressure & $\pm 0.11 L$ & $\begin{array}{l}\text { Figs. } 4.1-4.3, \text { A.1, and B. } 1 \text { and Ta- } \\
\text { bles } 4.1 \text { and } 4.2\end{array}$ \\
\hline$x_{S}$ & PIV & {$[ \pm 0.36 L, \pm 0.32 L, \pm 0.34 L]$} & $\begin{array}{l}\text { Figs. } 4.4,4.6-4.8,4.10, \text { A.2, } \\
\text { and A.6 and Tables } 4.1 \text { and } 4.2\end{array}$ \\
\hline$x_{S}$ & $T$ & {$\left[{ }_{-0.83}^{+0.92} L,{ }_{-1.52}^{+0.42} L,{ }_{-2.05}^{+0.38} L\right]$} & Figs. 4.11 and A. 6 and Table 4.2 \\
\hline$x_{S}$ & $h^{*}$ & {$\left[{ }_{-0.44}^{+0.33} L,{ }_{-0.26}^{+0.25} L,{ }_{-0.24}^{+0.20} L\right]$} & $\begin{array}{l}\text { Figs. } 4.13, \text { A. } 6 \text {, and B. } 2 \text { and Ta- } \\
\text { ble } 4.2\end{array}$ \\
\hline$x_{T}$ & Pressure & $\pm 0.38 \mathrm{~L}$ & $\begin{array}{l}\text { Figs. } 4.1-4.3, \text { A.1, and B. } 1 \text { and Ta- } \\
\text { bles } 4.1 \text { and } 4.2\end{array}$ \\
\hline$x_{T}$ & PIV & $\pm 0.03 \mathrm{~L}$ & $\begin{array}{l}\text { Figs. } 4.4-4.10, \text { A.2, and A.6 } \\
\text { and Tables } 4.1 \text { and } 4.2\end{array}$ \\
\hline$x_{T}$ & $T$ & {$\left[{ }_{-0.49}^{+0.28} L,{ }_{-0.20}^{+0.24} L,{ }_{-0.21}^{+0.20} L\right]$} & Figs. 4.11 and A. 6 and Table 4.2 \\
\hline$x_{T}$ & $h^{*}$ & {$\left[{ }_{-0.24}^{+0.17} L,{ }_{-0.14}^{+0.20} L,{ }_{-0.13}^{+0.24} L\right]$} & Figs. 4.13 and A. 6 and Table 4.2 \\
\hline$x_{R}$ & Pressure & $\pm 0.38 L$ & $\begin{array}{l}\text { Figs. } 4.1-4.3, \text { A.1, and B. } 1 \text { and Ta- } \\
\text { bles } 4.1 \text { and } 4.2\end{array}$ \\
\hline$x_{R}$ & PIV & {$[ \pm 0.09 L, \pm 0.05 L, \pm 0.09 L]$} & $\begin{array}{l}\text { Figs. 4.4, 4.6-4.10, A.2, and A.6 } \\
\text { and Tables } 4.1 \text { and } 4.2\end{array}$ \\
\hline$x_{R}$ & $T$ & {$\left[{ }_{-0.14}^{+0.35} L,{ }_{-0.22}^{+0.16} L,{ }_{-0.32}^{+0.34} L\right]$} & Figs. 4.11 and A. 6 and Table 4.2 \\
\hline$x_{R}$ & $h^{*}$ & {$\left[{ }_{-0.09}^{+0.11} L,{ }_{-0.20}^{+0.10} L,{ }_{-0.43}^{+0.10} L\right]$} & Figs. 4.13 and A.6 and Table 4.2 \\
\hline
\end{tabular}

* $95 \%$ confidence interval

istic length for the flat plate and airfoil experiments, respectively. The kinematic viscosity depends on both ambient temperature and pressure, which are associated with relative uncertainties of $\pm 0.2 \%$ and $\pm 0.01 \%$, respectively. Combining these uncertainties yields an uncertainty in the kinematic viscosity of $\pm 0.2 \%$. The free-stream velocity within the test section is based on measurements of static pressure drop across the contraction, which was calibrated in an empty test section against a Pitot-static tube. The pressure uncertainty was dominated by transducer accuracy, which is $\pm 0.14 \%$ of full-scale, or $\pm 0.70 \mathrm{~Pa}$. Additional uncertainty was introduced in the calibration of the pressure transducer, where known input pressures were applied to the transducer with a Druck DPI 610 LP pressure calibrator and related to the output voltage. A line of best fit was applied to the data, where the root-mean-square error of the linear fit introduced an uncertainty of $\pm 0.16 \mathrm{~Pa}$. Combining the uncertainties in measured pressure with Eq. D.1 results in a total uncer- 
Table D.2: Summary of uncertainty estimates for airfoil experiments.

\begin{tabular}{|c|c|c|c|}
\hline Quantity & Conditions & Uncertainty $^{*}$ & Applies to \\
\hline $\operatorname{Re}_{c}$ & & $\pm 2 \%$ & \\
\hline$T$ & & $\pm 0.04^{\circ} \mathrm{C}$ & Figs. 5.7-5.9 and A.7-A.9 \\
\hline$h^{*}$ & & {$[ \pm 0.005, \pm 0.009]$} & Figs. 5.10, A.10, and A.11 \\
\hline$x_{S}$ & PIV & {$[ \pm 0.04 c, \pm 0.03 c]$} & $\begin{array}{l}\text { Figs. 5.1, 5.3-5.5, and } 5.7 \text { and Tables } 5.1 \\
\text { and } 5.2\end{array}$ \\
\hline$x_{S}$ & $T$ & {$\left[ \pm 0.03 c,{ }_{-0.02}^{+0.05} c\right]$} & Figs. 5.8, A.12, and A.13 and Table 5.2 \\
\hline$x_{S}$ & $h^{*}$ & {$\left[{ }_{-0.06}^{+0.03} \mathrm{c},{ }_{-0.08}^{+0.04} \mathrm{c}\right]$} & Figs. 5.10, A.12, and A.13 and Table 5.2 \\
\hline$x_{S}$ & $h$ & {$\left[{ }_{-0.04}^{+0.11} c,{ }_{-0.04}^{+0.08} \mathrm{c}\right]$} & Fig. 5.13 and Table 5.2 \\
\hline$x_{T}$ & PIV & {$[ \pm 0.01 c, \pm 0.02 c]$} & Figs. 5.1-5.7 and Tables 5.1 and 5.2 \\
\hline$x_{T}$ & $T$ & {$[ \pm 0.03 c, \pm 0.02 c]$} & Figs. 5.8, A.12, and A.13 and Table 5.2 \\
\hline$x_{T}$ & $h^{*}$ & {$[ \pm 0.02 c, \pm 0.02 c]$} & Figs. 5.10, A.12, and A.13 and Table 5.2 \\
\hline$x_{T}$ & $h$ & {$\left[{ }_{-0.09}^{+0.10} c,{ }_{-0.09}^{+0.08} c\right]$} & Fig. 5.13 and Table 5.2 \\
\hline$x_{R}$ & PIV & {$[ \pm 0.04 c, \pm 0.02 c]$} & Figs. 5.1-5.7 and Tables 5.1 and 5.2 \\
\hline$x_{R}$ & $T$ & {$[ \pm 0.02 c, \pm 0.02 c]$} & Figs. 5.8, A.12, and A.13 and Table 5.2 \\
\hline$x_{R}$ & $h^{*}$ & {$[ \pm 0.02 c, \pm 0.02 c]$} & Figs. 5.10, A.12, and A.13 and Table 5.2 \\
\hline$x_{R}$ & $h$ & {$\left[{ }_{-0.05}^{+0.08} c,{ }_{-0.06}^{+0.10} c\right]$} & Fig. 5.13 and Table 5.2 \\
\hline
\end{tabular}

* $95 \%$ confidence interval

tainty in pressure of $\pm 0.72 \mathrm{~Pa}$. This uncertainty in the static pressure drop measurement is propagated to the free-stream dynamic pressure using Eq. D.2 and the relationship between contraction pressure drop and dynamic pressure determined in the test section calibration. The resulting uncertainty in free-stream dynamic pressure was calculated to be $\pm 3.1 \%$ at the lowest velocity investigated, corresponding to the highest relative uncertainty. Using this value and an uncertainty in air density of $\pm 0.2 \%$, the uncertainty in free-stream velocity was calculated to be less than $1.5 \%$ for all experimental conditions. Combining the uncertainty in free-stream velocity with those of kinematic viscosity and characteristic length using Eq. D.2 yields a relative uncertainty in Reynolds number of less than $\pm 2 \%$ for all flow conditions investigated.

\section{D.2 Uncertainty in Pressure Measurements}

Static pressure distributions on the flat plate were measured with Setra model 239 highaccuracy pressure transducers, with a full range of $\pm 250 \mathrm{~Pa}$. As previously mentioned, the error associated with measured pressure is due to both the transducer accuracy (which accounts for hysteresis, non-linearity, and non-repeatability), and the pressure transducer calibration, resulting in a total pressure error of $\pm 0.72 \mathrm{~Pa}$. As 20,000 samples were performed 
for each pressure measurement, the error associated with temporal variance is assumed to be negligible. The distributions of static pressure are presented non-dimensionalized by the dynamic pressure, such that the error associated with dynamic pressure must also be considered. For each Reynolds number, the error in measured pressure is combined with the error in dynamic pressure, resulting in a maximum total uncertainty in $C_{P}$ of \pm 0.03 at $\operatorname{Re}_{L}=27,000$, and \pm 0.02 at $\operatorname{Re}_{L}=36,000$ and 45,000.

\section{D.3 Uncertainty in PIV Measurements}

Quantifying the uncertainty associated with PIV is difficult due to the number of contributing factors. As the estimated characteristic locations are the only quantitative values of interest in this study, only the factors which contribute significantly to these estimations are considered. The primary sources of uncertainty associated with characterizing the laminar separation bubble are: i) random errors in measured PIV velocity which are propagated to the mean dividing streamline estimates, and ii) uncertainty in the exact location of the model surface in PIV images.

Random errors associated with PIV measurements can be caused by a number of factors, such as out of plane particle motion resulting in a loss of particles between frames, camera noise levels, and high velocity gradients [126]. In this study, the random error associated with PIV measurements is computed using the particle disparity method built into DaVis 8 [126]. For the flat plate experiments this was found to be approximately $\pm 1 \%$ of the free-stream velocity near separation, and less than $\pm 2 \%$ in the aft portion of the bubble. Similarly for the airfoil experiments, the random PIV error was found to be approximately $\pm 2 \%$ of the free-stream velocity in the fore portion of the bubble, and less than $\pm 4 \%$ in the aft portion of the bubble. The error in measured velocity was added and subtracted from the time-averaged velocity fields, and the characteristic locations were estimated for each case, with the most extreme location used as the uncertainty bound for each characteristic location.

Another factor which significantly contributed to the estimated characteristic locations was uncertainty in the exact location of the model surface in PIV images. The model surface was located in PIV calibration images with an associated uncertainty of $\pm 0.20 \mathrm{~mm}$. For each flow condition, the model surface was shifted up and down by the wall location uncertainty, and the locations of mean separation and reattachment were identified. The most extreme locations estimated from the intersection of the mean dividing streamline with the model surface established the associated uncertainty limits. Another source of uncertainty associated with PIV measurements applies only to the estimated reattachment location at $\mathrm{Re}_{c}=80,000$ in the airfoil experiments due to failure in capturing reattach- 
ment within the field of view. Consequently, the linear fit applied to the mean dividing streamline in the aft portion of the bubble at $\mathrm{Re}_{c}=80,000$ was based on fewer points than at $\operatorname{Re}_{c}=120,000$, such that the linear fit introduced an additional uncertainty of $\pm 0.02 c$. Considering uncertainty contributions from each of the aforementioned sources, the total uncertainty associated with the characteristic location estimates was computed with Eq. D.1, with the resulting values summarized in Tables D.1 and D.2 for the flat plate and airfoil experiments, respectively.

\section{D.4 Uncertainty in Thermal Measurements}

The uncertainty associated with characterization of the laminar separation bubble using thermal techniques is comprised of contributions from: i) random errors due to the infrared camera thermal sensitivity, ii) error in averaged quantities associated with spanwise and temporal variations, iii) error associated with spatial calibration of the infrared camera, and iv) reduced resolution introduced by data smoothing. These sources are each discussed and quantified, yielding a total estimated uncertainty associated with each characteristic location summarized in Tables D.1 and D.2 for the flat plate and airfoil experiments, respectively.

Since the Optris PI640 camera has a thermal sensitivity of $75 \mathrm{mK}$, the uncertainty associated with the temperature resolution is $\pm 37.5 \mathrm{mK}$. Additionally, the streamwise profiles used for separation bubble characterization were based on time- and spanwise-averaged measurements, such that the spatial and temporal variation should be considered. To do so, the standard deviation of surface temperature was computed over time, and over the spanwise direction, at each pixel location. The random errors contributions by spatial and temporal fluctuations were quantified as twice the standard deviation divided by the square root of the number of samples [123-125]. Due to the large dataset acquired, these contributions were found to be negligible compared to the thermal sensitivity of the camera, such that the uncertainty associated with each surface temperature measurement is $\pm 37.5 \mathrm{mK}$. Bias errors due to accuracy of the infrared camera are not considered here as they do not affect characterization of the laminar separation bubble.

Once time- and spanwise-averaged profiles of surface temperature were computed, it was found that the thermal sensitivity of the infrared camera introduced a fluctuating component to the temperature profile. In this regard, assuming random distribution of errors in space, a spatial filter of kernel size $N$ was applied to the surface temperature distribution to minimize the propagation of random spatial temperature variations into gradient estimates. However, the spatial filter reduces the spatial resolution, limiting it to $\pm \frac{1}{2}$ filter width. A spatial filter of 5px was applied to all distributions of time- and spanwise-averaged 

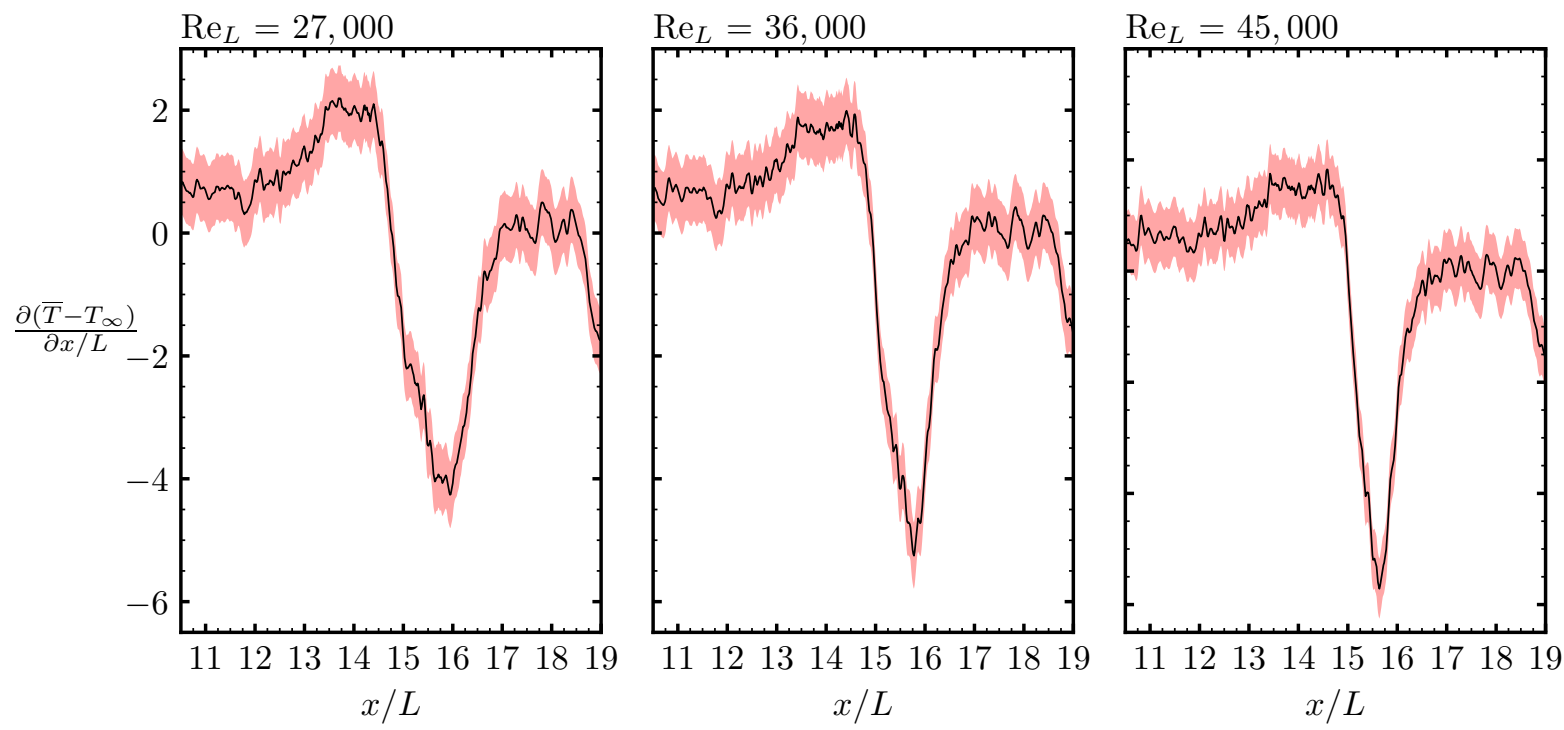

Figure D.1: Time- and spanwise-averaged distribution of the streamwise gradient of surface temperature for each highlighted Reynolds number on the flat plate with associated uncertainty bounds.

surface temperature and $h^{*}$, with an additional 5px filter applied to their gradient profiles. This allowed for a significant reduction in random error propagation to the gradient profiles, while only contributing additional uncertainty due to spatial resolution reduction of $\pm 0.07 \mathrm{~L}$ and $\pm 0.01 \mathrm{c}$ to the characteristic locations determined from the variation of surface temperature and $h^{*}$, and a spatial uncertainty of $\pm 0.10 H$ and $\pm 0.02 c$ to the characteristic locations determined from the variation of their streamwise gradient, for the flat plate and airfoil experiments, respectively. With the spatial filter applied, the combined uncertainty in surface temperature and $h^{*}$ is relatively small (Tables D.1 and D.2) and is not included in the corresponding plots for clarity. The uncertainty is propagated into the numerical gradient estimation by a factor of $\frac{1}{\sqrt{2} \Delta x}$. The streamwise gradients of surface temperature and $h^{*}$ with associated uncertainty bounds are shown for the flat plate and airfoil experiments in Figs. D.1 and D.2 and Figs. D.3 and D.4, respectively.

The final source of uncertainty associated with the temperature-based estimations is due spatial calibration of the infrared camera with the model surface. Markers of known position were placed on the surface of each model and the locations were recorded in the acquired infrared images. For the airfoil model, the coordinates were then transformed to a surface attached system to match the PIV results. The corresponding error due to spatial calibration of the infrared camera was determined to be $\pm 0.05 \mathrm{~L}$ and $\pm 0.01 \mathrm{c}$ for the flat plate and airfoil experiments, respectively.

In order to quantify the uncertainty associated with each characteristic location esti- 

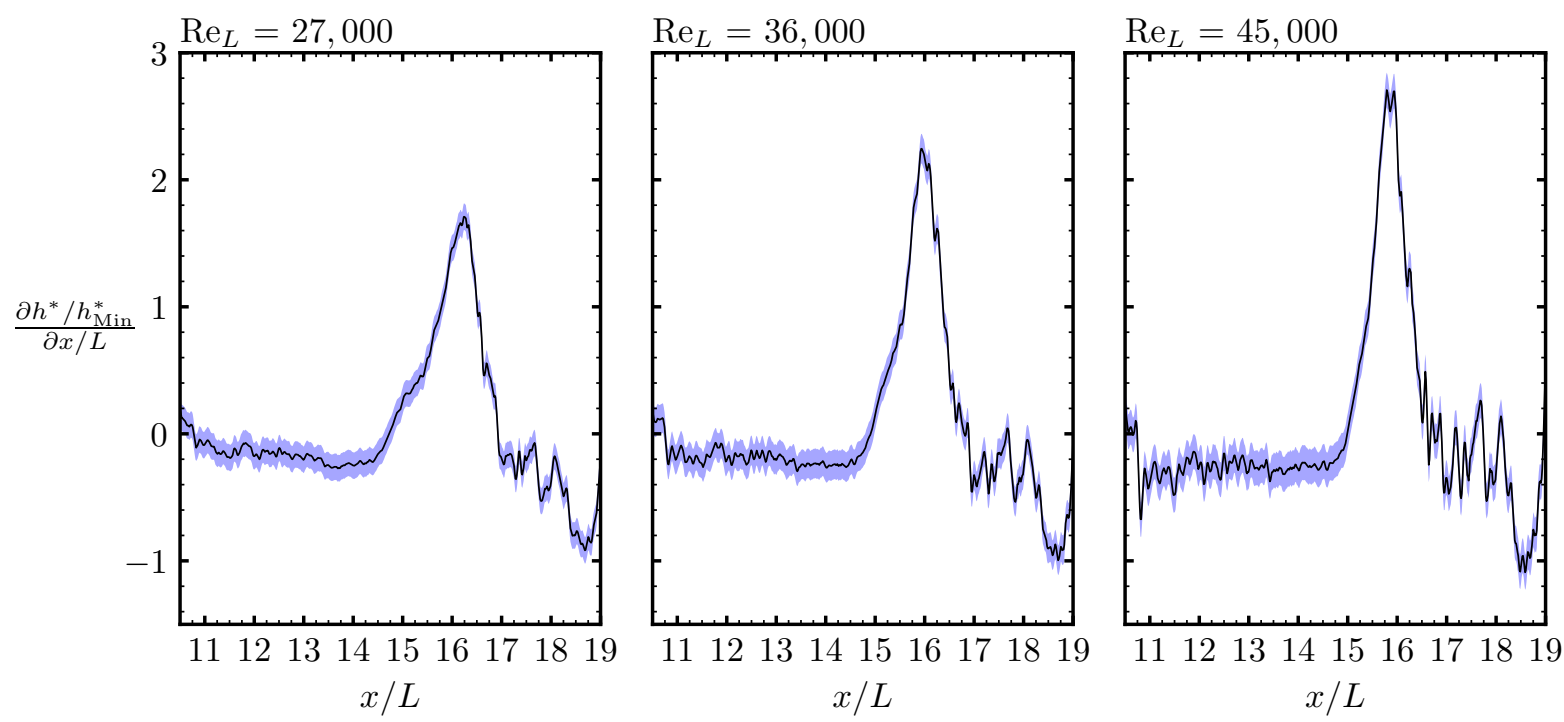

Figure D.2: Time- and spanwise-averaged distribution of the streamwise gradient of $h^{*}$ for each highlighted Reynolds number on the flat plate with associated uncertainty bounds.

mated from an extremum location, a methodology shown in Fig. D.5 was employed. The uncertainty for characteristic locations estimated at local maxima was estimated based on the region where the upper uncertainty bound is above the absolute maximum of the lower uncertainty bound. Similarly for the characteristic locations estimated at local minima, the uncertainty was estimated based on the region where the lower uncertainty bound is below the absolute minimum of the higher uncertainty bound. For the separation location estimated from distributions of $h^{*}$ in the flat plate experiments, where lines of best fit were used to find the minimum gradient location, the uncertainty bounds were determined with the most upstream and downstream intersection locations of linear lines passing through the streamwise gradient of $h^{*}$, within the associated uncertainty limits. For each characteristic location, the total uncertainty was estimated by combining the aforementioned contributions using Eq. D.1.

To quantify the uncertainty associated with the transient-based estimations, a similar approach as discussed above was implemented. The error in the measured temperature due to the infrared camera thermal sensitivity was propagated to $\frac{T-T_{\infty}}{T_{0}-T_{\infty}}$, allowing for the error associated with the exponential fit to be quantified. The upper and lower bounds of the exponential fit established the uncertainty associated with the distribution of $h / h_{\text {Min }}$, which is shown in Fig. D.6. Due to the increased fluctuations associated with the transient-based measurements, a spatial filter with a kernel of 10px was applied to the $h / h_{\text {Min }}$ profile and its streamwise gradient, resulting in spatial errors of $\pm 0.03 \mathrm{c}$ for separation and reattachment, and $\pm 0.06 \mathrm{c}$ for transition estimates. The uncertainty in each characteristic location 

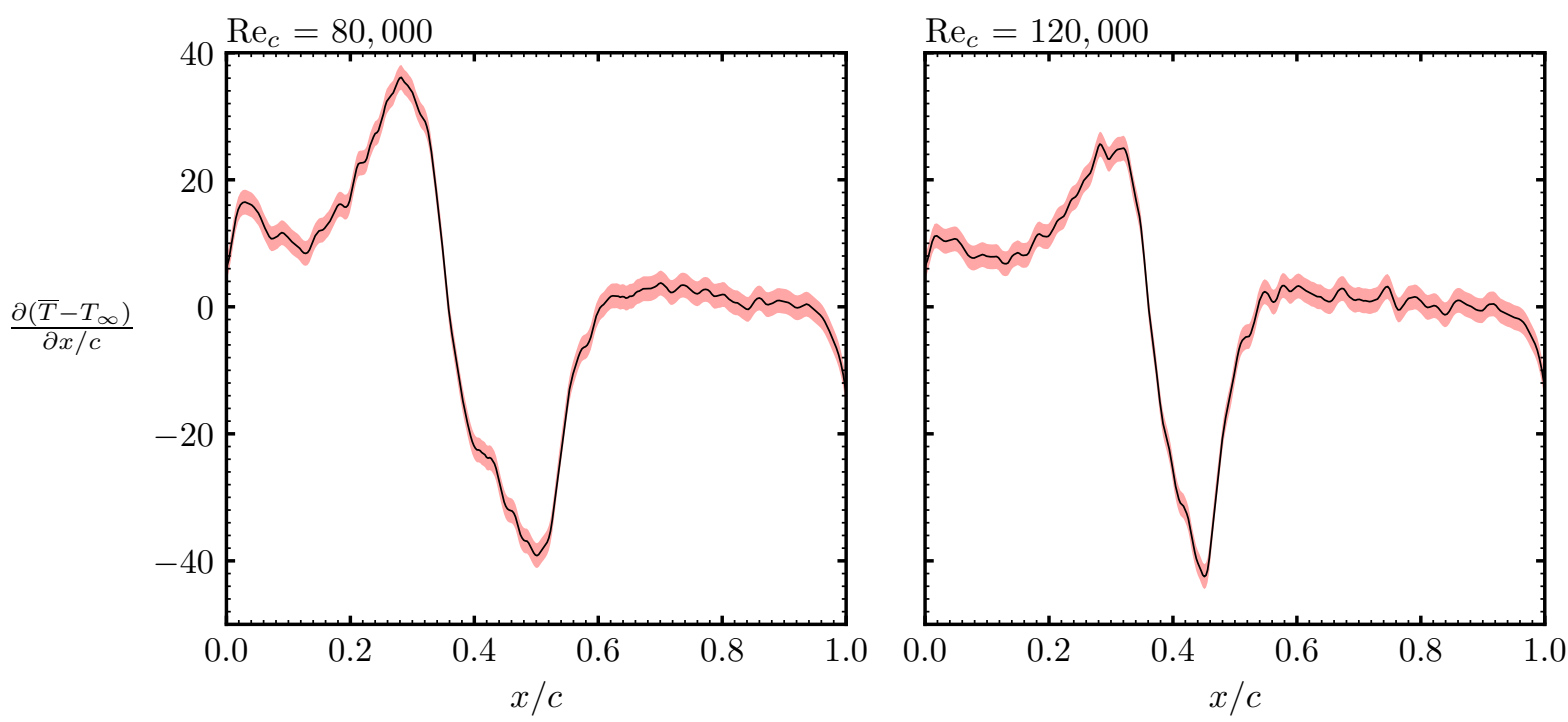

Figure D.3: Time- and spanwise-averaged distribution of the streamwise gradient of surface temperature for each highlighted Reynolds number on the airfoil with associated uncertainty bounds.

was estimated using the same methodology as for the time-averaged data, illustrated in Fig. D.5. These uncertainty estimates were combined with those from spatial filtering and the spatial calibration using Eq. D.1, with the total uncertainty for each characteristic location summarized in Table D.2. 

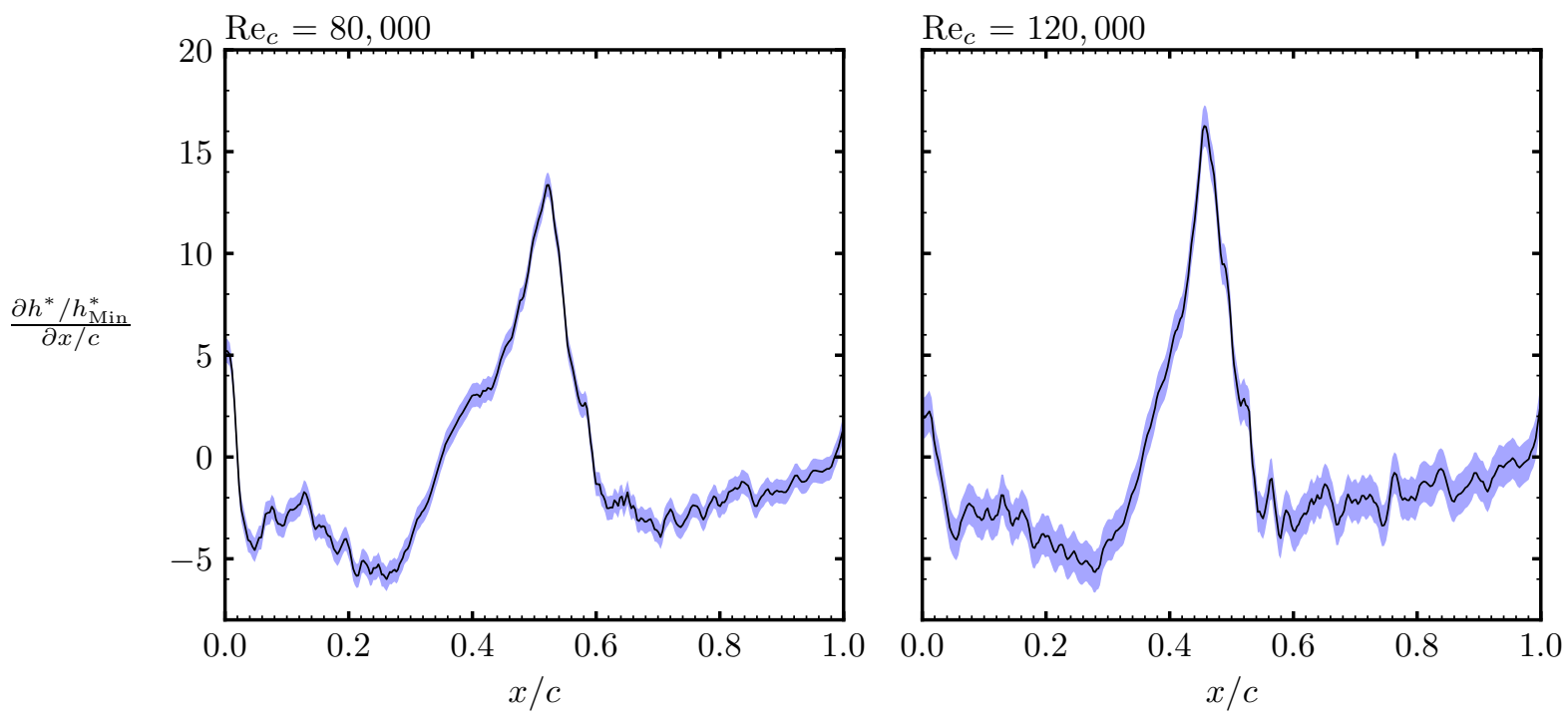

Figure D.4: Time- and spanwise-averaged distribution of the streamwise gradient of $h^{*}$ for each highlighted Reynolds number on the airfoil with associated uncertainty bounds.
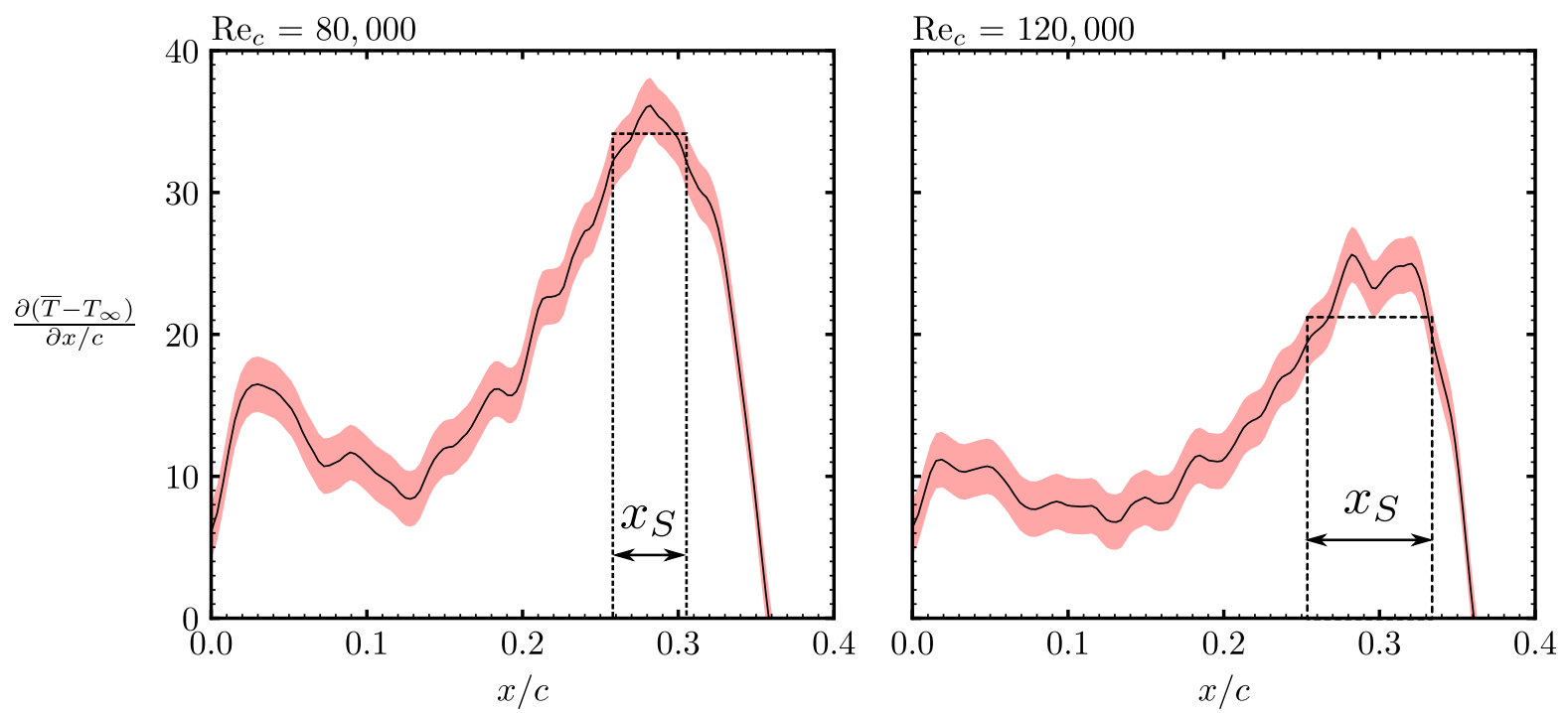

Figure D.5: Example of the methodology employed for estimating the uncertainty in each characteristic location shown for the separation estimate from surface temperature gradient profiles on the airfoil geometry. 

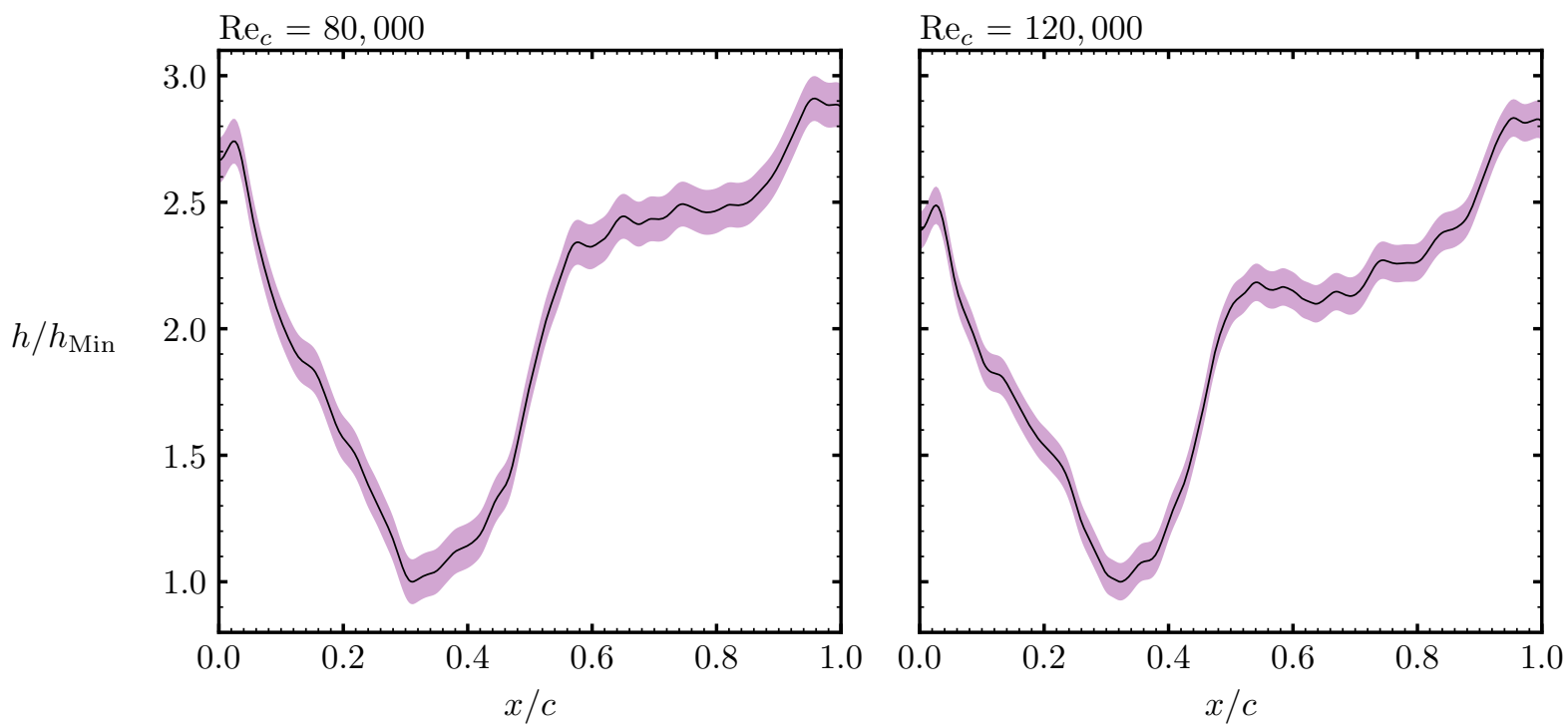

Figure D.6: Spanwise-averaged distribution of the convective heat transfer coefficient from transient-based measurements with associated uncertainty bounds.
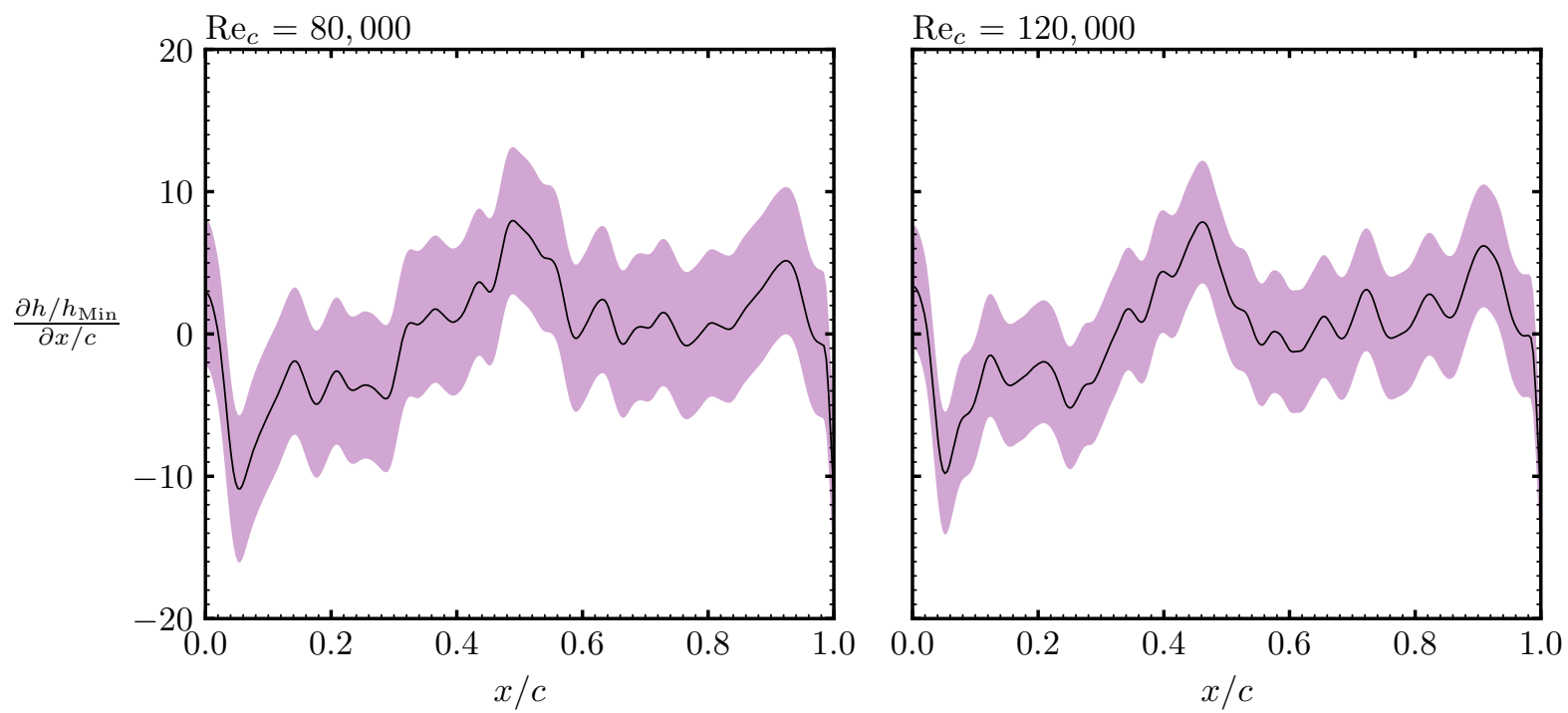

Figure D.7: Spanwise-averaged distribution of the streamwise gradient of convective heat transfer coefficient from transient-based measurements with associated uncertainty bounds. 